\title{
Syndesmotic Ankle Sprains in Large Males
}

\section{A Thesis}

\section{Presented to}

the faculty of the School of Engineering and

Applied Science

University of Virginia

\author{
in partial fulfillment \\ of the requirements for the degree \\ Master of Science
}

by

Alexander Ritz Mait

August 2017 


\section{APPROVAL SHEET}

This Thesis

is submitted in partial fulfillment of the requirements for the degree of Master of Science

Author Signature:

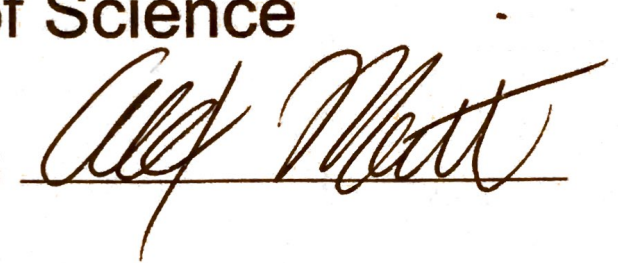

This Thesis has been read and approved by the examining committee:

Advisor: Dr. Richard W. Kent

Committee Member: Dr. Matthew B. Panzer

Committee Member: Dr. Robert S. Salzar

Committee Member: Dr. Jason L. Forman

Committee Member: Dr. M. Truitt Cooper

Committee Member:

Accepted for the School of Engineering and Applied Science:

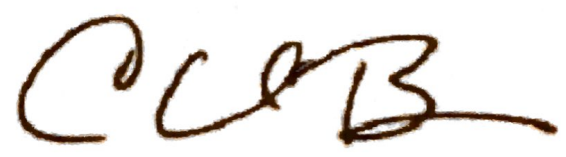

Craig H. Benson, School of Engineering and Applied Science 


\section{$\underline{\text { Acknowledgements }}$}

I owe thanks to several folks for their guidance and support during my years at the University of Virginia (UVA) while completing my Master of Science in Mechanical and Aerospace Engineering at the Center for Applied Biomechanics (CAB).

First, I must thank Dean James Aylor for welcoming me to the UVA community and introducing me to the outstanding scholars at CAB. Next, thanks to my advisor Professor Richard Kent for providing me the opportunity to flourish as a student, researcher, and person at CAB. I appreciate you entrusting me with such an important and challenging research project. Your guidance instilled a high level of intellectual curiosity in me which has undoubtedly facilitated my growth as an engineer and a scholar.

I must acknowledge the other members of the High Ankle Sprain research group, Professor Matthew Panzer, Dr. Jason Forman, and Dr. Bingbing Nie for their continuous support throughout my time at $\mathrm{CAB}$. From hours in the prep room, to improvements on test fixtures, to editing publications, and enhancing my presentation skills, your guidance undeniably helped me develop as a person, engineer, and researcher.

I would like to thank Dr. Robert Salzar, Dr. E. Meade Spratley, and Dr. Bronek Gepner for their valuable advice and feedback throughout my time at CAB. I received exceptional support from the CAB staff, including Sara Heltzel, Brian Overby, Joey White, Kevin Kopp, Meghan Darney, and Ted Miller, while performing my experimental work, for that I want to thank all of you.

I wish to thank my fellow CAB graduate students, especially Brandon Perry, Daniel Perez-Rapela, Lee Gabler, Adwait Mane, and John Paul Donlon, for making my time at CAB memorable. From coursework help to publication revisions to social gatherings, your support and friendship will be forever important and meaningful to me.

Lastly, but certainly not least, I must thank my parents Barbara and Russell Mait for their neverending love and support throughout my life. I would not be the person nor the scholar I am today without your encouragement and guidance. Your confidence in my dreams and abilities is humbling and I will be forever grateful to both of you. 


\begin{abstract}
$\underline{\text { Abstract }}$
Syndesmotic ankle sprains, i.e. injuries to the distal tibiofibular syndesmosis, are debilitating injuries often associated with arduous rehabilitation and recovery. External foot rotation, induced by internal rotation of the tibia, is hypothesized as the primary mechanism of these injuries, but the role of ankle flexion remains poorly understood for both injury patterns and tolerances. Furthermore, clinical observations include combinations of ligament and osseous injuries, with unclear links between causation and injury patterns.
\end{abstract}

The main objective of this thesis was to determine the injury pattern and sequence of ankle ligaments during excessive external foot rotation in varying ankle flexion postures: neutral, dorsiflexed $\left(15^{\circ}\right)$, and plantarflexed $\left(30^{\circ}\right)$. Specifically, this thesis aimed to address deficiencies in the literature by improving boundary conditions in experiments testing forceful external foot rotation in cadavers. Furthermore, this thesis aimed to determine an injury occurrence interval for syndesmotic ankle sprains, relative to the leg kinematic input of external foot rotation, in a neutral flexion posture. Changes in this injury interval were considered for varying flexion postures.

Nine matched-pair legs from non-senescent (47 $\pm 11.3 \mathrm{yrs}$.), large $(94.4 \pm 30.9 \mathrm{~kg}, 178.1 \pm 5.9 \mathrm{~cm})$ male cadaver legs were disarticulated at the knee joint. The proximal tibia was fixed and the fibula left unconstrained. External foot rotation was imposed by internally rotating the tibia while motion of the calcaneus and first metatarsal head were constrained. A nominal preload of $2 \mathrm{kN}$ was imposed along the tibia long-axis. Osteoligamentous injury timing was determined from acoustic sensors, strain gauges, force/moment measurements, and three-dimensional bony kinematics as external foot rotation, defined as calcaneus yaw relative to the tibia long-axis, was applied. Posttest necropsies were performed to identify injuries.

In neutral, syndesmotic injuries were identified in five of nine legs and deltoid injuries in nine of nine legs. Plantarflexion was protective of the syndesmotic ligaments, such that zero of four specimens sustained syndesmotic injury, yet three of four sustained a deltoid injury. Dorsiflexion focused loading through the syndesmosis during external rotation, such that four of four specimens sustained a syndesmotic injury and three of four a deltoid injury. As external rotation is applied, the talus creates lateral and posterior tibiofibular diastasis, eventually disrupting the syndesmotic ligaments. When legs are in neutral or dorsiflexion, this is exaggerated, yet protected against when combined with plantarflexion. This indicates plantarflexion does not widen the ankle mortise in the same way as in dorsiflexion or neutral, even under the same applied preload and external rotation.

These injury pattern and incidence results will inform future clinical diagnosis, care, and rehabilitation techniques for syndesmotic ankle sprains. Bone kinematic interactions and injury propagation and occurrence data will be used to validate a finite element ankle surrogate model. Ultimately, this model will act a design tool for assessing future countermeasures aimed at mitigating syndesmotic ankle sprains. 


\section{$\underline{\text { Table of Contents }}$}

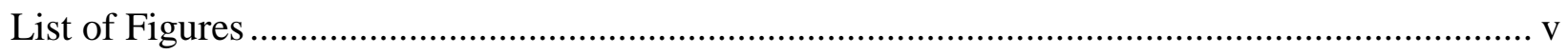

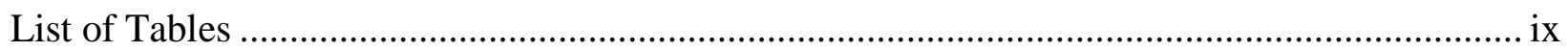

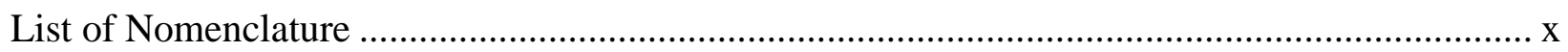

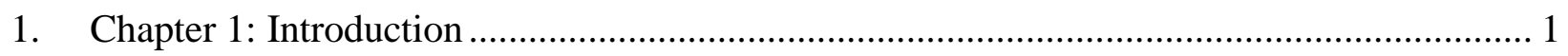

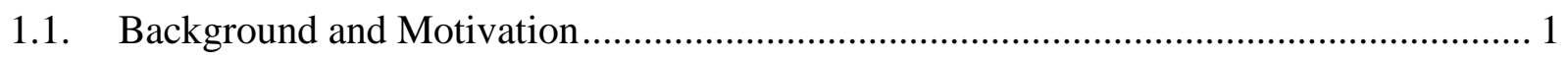

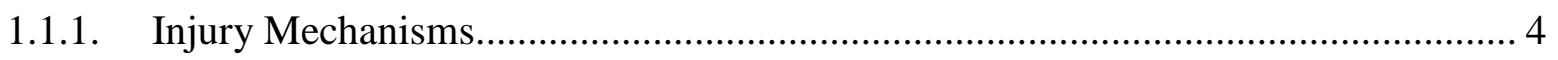

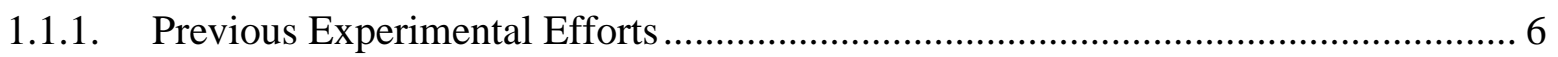

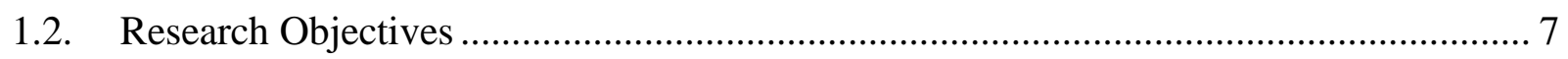

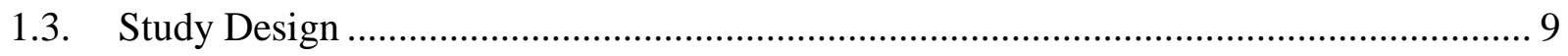

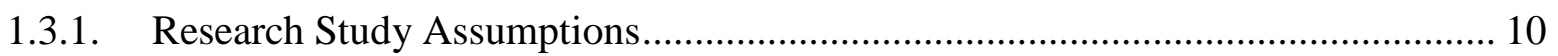

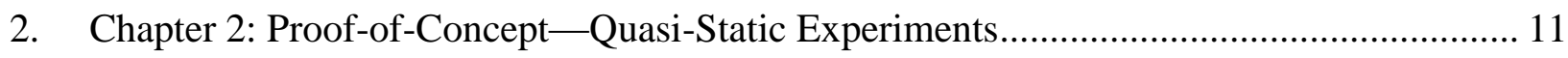

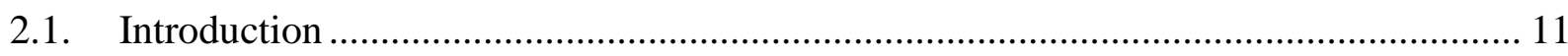

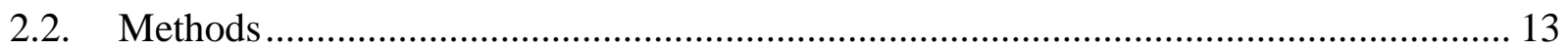

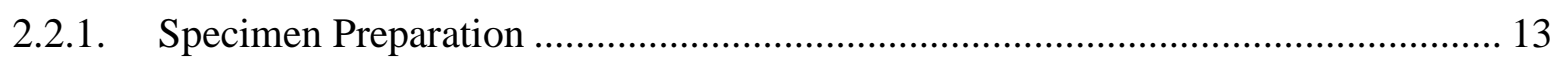

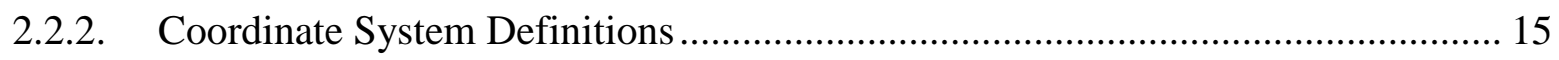

2.2.3. Calculating Bone Kinematics and Clinical Ankle Angles .................................... 20

2.2.4. Experimental Test Procedures, Boundary Condition Improvements, and Bone

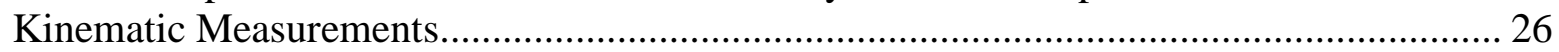

2.2.5. Experimental and Kinematic Measurement Accuracy Assessment......................... 30

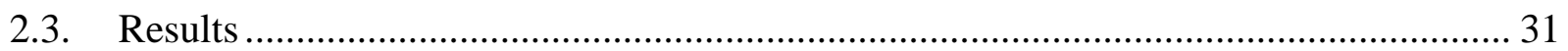

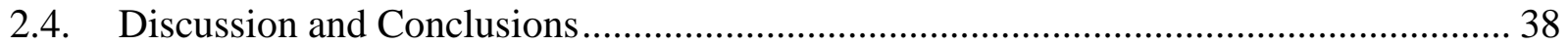

3. Chapter 3: Syndesmotic Injury Incidence and Effects of Ankle Flexion Posture ................. 42

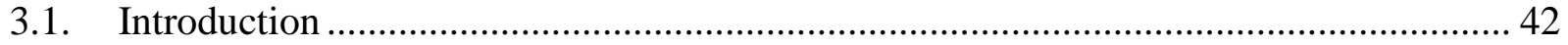

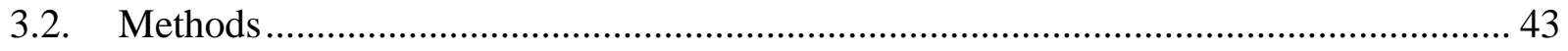

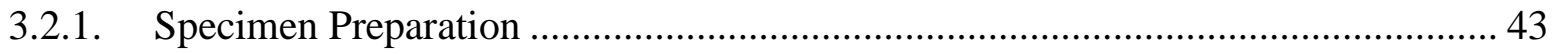

3.2.2. Test Apparatus and Loading Protocol................................................................... 47

3.2.3. Pilot Study: Adapting Quasi-Static Experimental Boundary Conditions ................ 49

3.2.4. Boundary Condition Improvements ................................................................ 53

3.2.5. Clinical Evaluation and Injury Event Sequence Determination ............................. 58

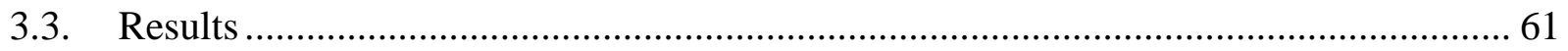

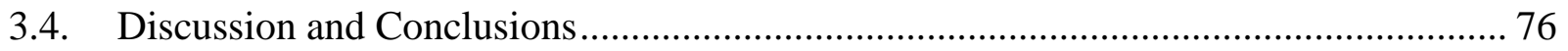

4. Chapter 4: Syndesmotic Injury Occurrence and Tibiofibular Diastasis ................................. 83 


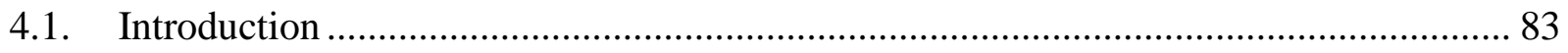

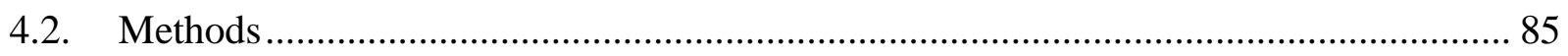

4.2.1. Tibiofibular Diastasis Determination............................................................ 85

4.2.2. Ankle Injury Occurrence Interval Determination .......................................... 88

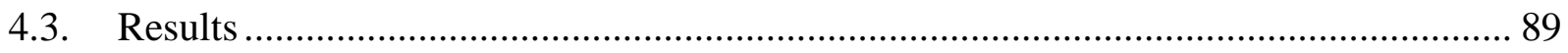

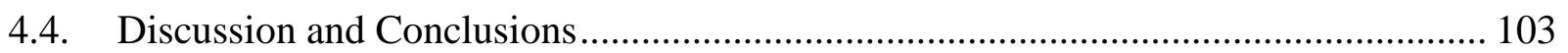

5. Chapter 5: Conclusions and Future Work................................................................ 111

5.1. Implications for Syndesmotic Ankle Sprains ........................................................ 111

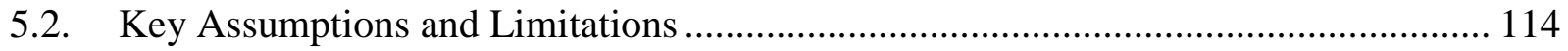

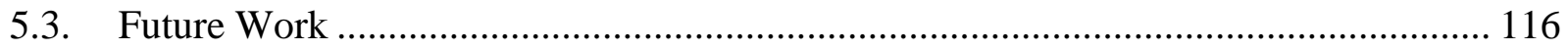

5.3.1. Experimental and Computational Improvements ........................................ 116

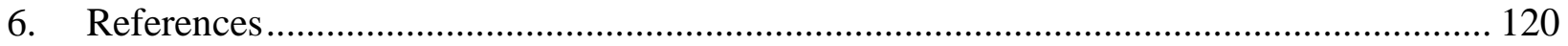




\section{List of Figures}

Figure 1: Gross rotations of the ankle permitted through the talocrural, subtalar, and syndesmosis joints (Mane, 2016; Shin et al., 2012).

Figure 2: Osteoligamentous ankle anatomy with pertinent bones and ligaments labeled in the Antero-Lateral (left), Medial (middle), and Posterior (right) views: adapted from Golano et al. (2010). 3

Figure 3: Hypothesized mechanism of injury on the playing field for American Football players where a lateral blow to the leg causes internal tibial rotation over a planted foot or when a player is lying prone on the ground (Left: Guise, 1976; Right: Norkus and Floyd, 2001)..... 5

Figure 4: Potted tibia of a specimen where wood screws are driven into the proximal shaft of the tibia and with Bondo body filler covering the anterior, posterior, and medial sides of the proximal tibia (left). Placement of the tibia and calcaneus external motion-tracking marker cubes as well as the open-faced mount around the calcaneus with threaded rods driven from lateral to medial (right). Several holes were placed on the lateral face of the calcaneus mount for threaded rods to pass through to accommodate different sizes of specimens, and threaded rods anchored into the medial face made of solid polyvinyl chloride.

Figure 5: External motion-tracking marker cube placement in the tibia, fibula, navicular, and talus bones as well as the calcaneus mount (left). X-ray image of marker cube placement confirmation (right) .......................................................................................... 15

Figure 6: Method to define the local coordinate system of the tibia (left leg) using geometry of the bone (inertial axes) and anatomical landmarks (plafond and medial malleolus).................. 18 Figure 7: Method to define the origin of the fibula (right leg) geometrically in Mimics 18.0. Left: eminence on the fibula most posterior and medial (i.e., closest to lateral-distal tibia), denoted as a red dot, with defined lateral malleolus in yellow. Right: indication of origin in lateral malleolus (yellow dot) with plane denoting the slice taken from fibula shaft to demark lateral malleolus.

Figure 8: Local bone coordinate system definitions for foot and ankle bones in the initially neutral posture. Tibia coordinate system is defined using anatomic landmarks and bone geometry. From this tibia coordinate system, coordinate systems on other foot and ankle bones can be defined at their respective centroids (except fibula, defined at centroid of lateral malleolus), where all other bone coordinate axes are parallel to those of the tibia in the neutral ankle posture. Motion of these other bones are measured relative to the tibia coordinate system.

Figure 9: Process for transforming the coordinate system of each bone to the tibia coordinate system. Global refers to the coordinate system of the laboratory. The solid arrows indicate reference frames and the dashed arrows indicate transformation matrices relating reference frames. Each mount-bone pair was assumed to be a single rigid body.

Figure 10: Top: Antero-Lateral view of the right leg in combined external rotation, dorsiflexion and eversion. Bottom: Definition of rotation, flexion, and version angles based on the coordinate systems of the tibia and calcaneus. Positive rotation, flexion, and version were external rotation, dorsiflexion, and eversion and negative rotation, flexion, and version were internal rotation, plantarflexion, and inversion. .....

Figure 11: Components extracted from $\mathbf{T}_{\text {Tib,Cal }}$ to calculate joint angles $(E R=$ external foot rotation, $\mathrm{EV}=$ ankle eversion, $\mathrm{DF}=$ ankle dorsiflexion). 
Figure 12: (Left) Experimental test rig, with a specimen in its neutral initial position, detailing each component or device used during testing as well as placement of these components in the rig. (Right) Schematic of the experimental boundary conditions with the locations of the input rotation and axial load. The global coordinate system (X-Y-Z) is defined at the proximal tibia connection to the potting cup and load cell (Z-axis is coincident with the tibia long-axis; $X$-axis inserts at the origin of the $\mathrm{Z}$ and $\mathrm{Y}$ axes and is aligned parallel to the second metatarsal). Fibula is unconstrained. Calcaneus transverse $(\mathrm{X}-\mathrm{Y})$ translation is permitted with the bi-directional rail system, and gross Z-translation is permitted with the linear rails. However, calcaneus rotation about the $\mathrm{X}$ and $\mathrm{Y}$ axes is constrained by the mount surrounding the bone.

Figure 13: Illustration of maximum deviation from the mean for points (distance, in $\mathrm{mm}$ ) and for the initial tibia local axes (angular, in ${ }^{\circ}$ ) determined by Mimics 18.0 and the experimenter...... 31

Figure 14: Calculated changes in linear $(\Delta x, \Delta y, \Delta z)$ and angular ( $\Delta$ yaw, $\Delta$ pitch, $\Delta$ roll) displacements of the fibula, calcaneus, talus, and navicular bones relative to the tibia coordinate system after ER. Neutral, everted, inverted, dorsiflexed, and plantarflexed initial ankle postures are reported. 34

Figure 15: Calculated changes in linear $(\Delta x, \Delta y, \Delta z)$ and angular ( $\Delta y a w, \Delta$ pitch, $\Delta$ roll) displacements of the fibula, calcaneus, talus, and navicular bones relative to the tibia coordinate system after IR. Neutral, everted, inverted, dorsiflexed, and plantarflexed initial ankle postures are reported.

Figure 16: Comparison of the three-dimensional reconstruction of bones (specimen 616L) for tests starting from the initially neutral, everted, or dorsiflexed postures, then externally rotated. Reconstructions of bones begin with the leg axially compressed (110 N preload)................... 37 Figure 17: Specimen preparation photos indicating the potting method (left) and kinematic marker placements in the ankle bones and strain gauge attachment on the tibia (right). 45 Figure 18: Initially neutral posture specimen test apparatus and corresponding boundary condition schematics for the BC:B (calcaneus in fixed orientation) test fixture used in Runs 1-3 (top) and the $\mathrm{BC}: \mathrm{C}$ (calcaneus free to invert/evert) test fixture used in Runs 4-9 (bottom)........ 51 Figure 19: Specimen in the test fixture for the injurious pilot study (Runs 1-3) with BC:B where the leg was positioned in an artificially plantarflexed posture (left). The foot was not engaged by the test fixture in $\mathrm{BC}: \mathrm{B}$ during applied rotation, thus allowing the talus and foot to droop (right).

Figure 20: Specimen test apparatus and corresponding boundary condition schematics for the initially plantarflexed (BC:D, Runs 10-13) ankle posture tests (top) and initially dorsiflexed (BC:E, Runs 14-17) ankle posture tests (bottom) ............................................................... 56 Figure 21: Dissection photographs documenting diagnosed injuries in the ankle (Example: Run

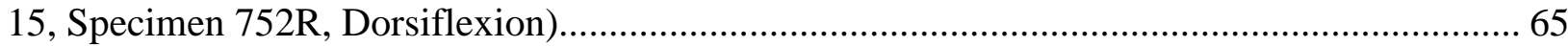
Figure 22: Injury event timing example (Run 15, Specimen 752R, Dorsiflexion) plots for (top) moment and strain and (bottom) moment and acoustic data traces. Injury events are highlighted: Event 1 in a yellow box, Events 2 and 3 in a purple box, and Events 4 and 5 in a green box. Note: Tibia LC - Mz refers to the reaction moment measured by the load cell fixed to the tibia plateau, Instron Rotation refers to the input rotation cycle, Microphone refers to the external microphone, and Acoustic Emission refers to the bone-mounted (on fibula) microphone acoustic sensor...... 66 Figure 23: Injury event timing example (Run 15, Specimen 752R, Dorsiflexion) plots for kinematic data traces of bone-specific (left) rotations and (right) translations. All data indicates the change in orientation or position of each bone in the tibia coordinate system. Injury events are highlighted: Event 1 in a yellow box, Events 2 and 3 in a purple box, and Events 4 and 5 in a 
green box. Note: Tibia LC - Mz refers to the reaction moment measured by the load cell fixed to the tibia plateau and Instron Rotation refers to the input rotation cycle. 68

Figure 24: Representative images of injury event sequences for (top) neutral test with calcaneus in fixed orientation (Run 1, Specimen 696R), (top-middle) neutral test with calcaneus free to invert/evert (Run 9, Specimen 757L), (bottom-middle) initially dorsiflexed test (Run 15, Specimen 752R), and (bottom) initially plantarflexed test (Run 12, Specimen 615R). Medial, Antero-Lateral, and Posterior views are shown with pertinent ankle ligaments and bones labelled. Boxes on the right detail the injury events, with circles in the illustrations highlighting these events.

Figure 25: Illustration of components from fibula displacement vector (in tibia coordinate system) in three-dimensional reconstructions of tibia and fibula. Anterior view illustrates (white arrow) the lateral and inferior displacement components and lateral view illustrates the posterior component.

Figure 26: Injury intervals, relative to external foot rotation, for injuries to the syndesmotic (i.e., ATiFL, IOM, and Weber B/C fibula fractures), deltoid (i.e., superficial and deep deltoid complexes), and lateral (i.e. ATaFL, PTaFL, CFL, and Weber A fibula fractures) ankle regions. Sensitivity to initial ankle flexion posture is indicated among initially plantarflexed (top), neutral (middle), and dorsiflexed (bottom) legs.

Figure 27: Plot of components of defined fibula displacement vector and magnitude of displacement vector relative to external foot rotation in the non-matched pair initially neutral specimen (Run 1). Intervals of displacement are indicated around the external foot rotation timing for diagnosed syndesmotic injuries.

Figure 28: Plot of components of defined fibula displacement vector and magnitude of displacement vector relative to external foot rotation in matched pair specimens for the initially neutral and plantarflexed postures. Intervals of displacement are indicated around the external foot rotation timing for diagnosed syndesmotic injuries.

Figure 29: Plot of components of defined fibula displacement vector and magnitude of displacement vector relative to external foot rotation in matched pair specimens for the initially neutral and dorsiflexed postures. Intervals of displacement are indicated around the external foot rotation timing for diagnosed syndesmotic injuries.

Figure 30: Difference between fibula and tibia yaw in the global coordinate system plotted against external foot rotation in the non-matched pair initially neutral specimen (Run 1). Intervals of rotation difference are indicated around the external foot rotation timing for diagnosed syndesmotic injuries.

Figure 31: Plot of fibula and tibia yaw difference in the global coordinate system relative to external foot rotation in matched pair specimens for the initially neutral and plantarflexed postures. Intervals of rotation difference are indicated around the external foot rotation timing for diagnosed syndesmotic injuries. .98

Figure 32: Plot of fibula and tibia yaw difference in the global coordinate system relative to external foot rotation in matched pair specimens for the initially neutral and dorsiflexed postures. Intervals of rotation difference are indicated around the external foot rotation timing for diagnosed syndesmotic injuries.

Figure 33: Injury intervals, relative to fibula displacement vector magnitude, for injuries to the syndesmosis (i.e., ATiFL, IOM, and Weber B/C fibula fractures. Sensitivity to initial ankle flexion posture is indicated among initially neutral (top) and dorsiflexed (bottom) legs. Syndesmotic injury did not occur in initially plantarflexed specimens. 101 
Figure 34: Injury intervals, relative to tibia and fibula yaw difference, for injuries to the syndesmosis (i.e., ATiFL, IOM, and Weber B/C fibula fractures. Sensitivity to initial ankle flexion posture is indicated among initially neutral (top) and dorsiflexed (bottom) legs.

Syndesmotic injury did not occur in initially plantarflexed specimens. 103

Figure 35: Computational finite element model ankle surrogate which should be used to test the viability of future countermeasure designs aimed at mitigating syndesmotic ankle sprains..... 117 


\section{List of Tables}

Table 1: Previous ankle rotation studies with mined data for specimen age, vertical preload, measured torque, and injuries at failure. Experimental deficiencies for each study are detailed... 6 Table 2: Whole-body specimen anthropometry (gender, age at time of death, height, and weight) for the three specimens tested in the small-scale, proof-of-concept study. ............................... 13 Table 3: Definitions of ankle rotation, flexion, and version angles: clinical vs. geometric (based on tibia and calcaneus local coordinate systems). ......................................................... 16 Table 4: Absolute and percent differences for repeated ER tests on each specimen. Differences among the linear and angular displacements from the fibula, calcaneus, talus, and navicular are

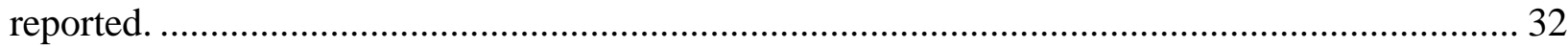

Table 5: Error estimation with resultant deviations from the mean for geometric coordinate system definition. Centroids of fibula, calcaneus, talus, and navicular calculated automatically by Mimics 18.0. Tibia z-axis calculated automatically by Mimics 18.0, but origin and medial malleolus points selected by experimenter (repeated selection of these points 5 times)............ 33 Table 6: Specimen whole-body anthropometric data with corresponding test input conditions. Cadaver anthropometric data is compared to that the NFL average player in 2014 (Manfred, 2014).

Table 7: Summary of instrumentation data channels measured in all Neutral Tests. Note: the instrumentation used in Runs 7-9 were repeated for the tests in initial plantarflexion (Runs 10-

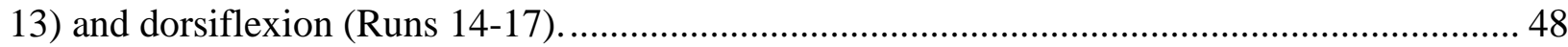
Table 8: Summary of boundary condition evolution used in applied foot rotation experiments. Iterations of boundary condition changes are detailed from the quasi-static tests to injury tests in various initial ankle flexion postures. .......................................................................... 58

Table 9: Injury event sequences for initially Neutral tests, Runs 1-3 (BC:B)........................ 61

Table 10: Injury event sequences for initially Neutral tests, Runs 4-9 (BC:C), ...................... 62

Table 11: Injury event sequences for initially Plantarflexed tests, Runs 10-13 (BC:D)............. 63

Table 12: Injury event sequences for initially Dorsiflexed tests, Runs 14-17 (BC:E). .............. 64 Table 13: Example (Run 15, Specimen 752R, Dorsiflexion) injury event sequence determination using all diagnostic tools (kinetics, kinematics, strain, and audio). Corresponding injuries are

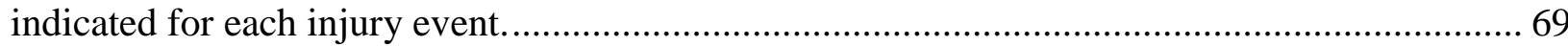

Table 14: Injury incidence for clinically significant documented injuries in the initially neutral (n $=9)$, plantarflexed $(n=4)$, and dorsiflexed $(n=4)$ ankle postures. .................................... 70

Table 15: Injury timings relative to external foot rotation (ER) measured during tests (Runs 1-3, $\mathrm{BC}: \mathrm{B})$ in the initially neutral ankle flexion posture ................................................... 73 Table 16: Injury timings relative to external foot rotation (ER) measured during tests (Runs 4-9, $\mathrm{BC}: \mathrm{C})$ in the initially neutral ankle flexion posture. 74 Table 17: Injury timings relative to external foot rotation (ER) measured during tests (Runs 10$13, \mathrm{BC}: \mathrm{D})$ in the initially plantarflexed ankle flexion posture. 75 Table 18: Injury timings relative to external foot rotation (ER) measured during tests (Runs 14$17, \mathrm{BC}: \mathrm{E})$ in the initially dorsiflexed ankle flexion posture. 


\section{List of Nomenclature}

Ligament Naming Convention:

Lateral Ligaments

ATaFL - Anterior Talofibular Ligament

CFL - Calcaneofibular Ligament

PTaFL - Posterior Talofibular Ligament

Medial Ligaments

Superficial deltoid complex - Tibionavicular Ligament (TiNL), Tibiospring Ligament, Anterior Tibiotalar Ligament (ATiTaL)

Deep deltoid complex - Two components: one branching from tibia to talus, one branching from tibia to calcaneus

Syndesmotic Ligaments

ATiFL - Anterior Tibiofibular Ligament

IOM - Interosseous Membrane

PTiFL - Posterior Tibiofibular Ligament

Ankle Motion Abbreviations:

IR - Internal Foot Rotation

ER - External Foot Rotation

DF - Dorsiflexion

PF - Plantarflexion

EV - Eversion

IV - Inversion

Unit and Mathematical Abbreviations:

$\mathrm{N}$ - Newtons

$\mathrm{kN}$ - Kilonewtons

$\mathrm{m}-$ Meters

$\mathrm{cm}-$ Centimeters

$\mathrm{mm}$ - Millimeters

yr. - Age in years

$\mathrm{kg}$ - Kilograms

$\mathrm{N}-\mathrm{m}$ - Newton-meters

$\mu \mathrm{s}$ - Micro-strain

$\mathrm{mV}$ - Millivolts

${ }^{\circ}$ - Degrees

$\Delta$ - Change in (de-biased)

$\sqrt{ }$ - Square Root

T - Transformation Matrix

$\mathbf{P}$ - Position Vector 


\section{Chapter 1: Introduction}

\subsection{Background and Motivation}

Human ankles are complicated mechanical structures featuring two main osteoligamentous joints: the talocrural ('ankle') joint which articulates between the tibia, fibula, and talus; and the talocalcaneal ('subtalar') joint which articulates between the talus and calcaneus (Funk, 2011; Kaumeyer and Malone, 1980; Leardini et al., 2000; Norkus and Floyd, 2001; Sclafani, 1985). The talocrural joint is a uniaxial, modified-hinge joint formed with the talar dome fitting between the medial and lateral malleoli, which define the ankle mortise (Norkus and Floyd, 2001). Whereas, the talocalcaneal joint, inferior to the talocrural joint, is a gliding joint between the talus and calcaneus joined by articular capsule and ligaments (Norkus and Floyd, 2001). Superior to the talocrural joint is the distal tibiofibular syndesmosis joint which is described as a synovial joint permitting articulations between the distal tibia and fibula (Bartonicek, 2003; Peña and Coetzee, 2006). Ligaments constrain these joints and their associated bones but permit gross physiological ranges of motion (Figure 1) such as ankle eversion and inversion, dorsiflexion and plantarflexion, and external and internal rotation (Funk, 2011; Mane, 2016; Nigg et al., 1990; Norkus and Floyd, 2001; Roaas and Andersson, 1982; Shin et al., 2012).

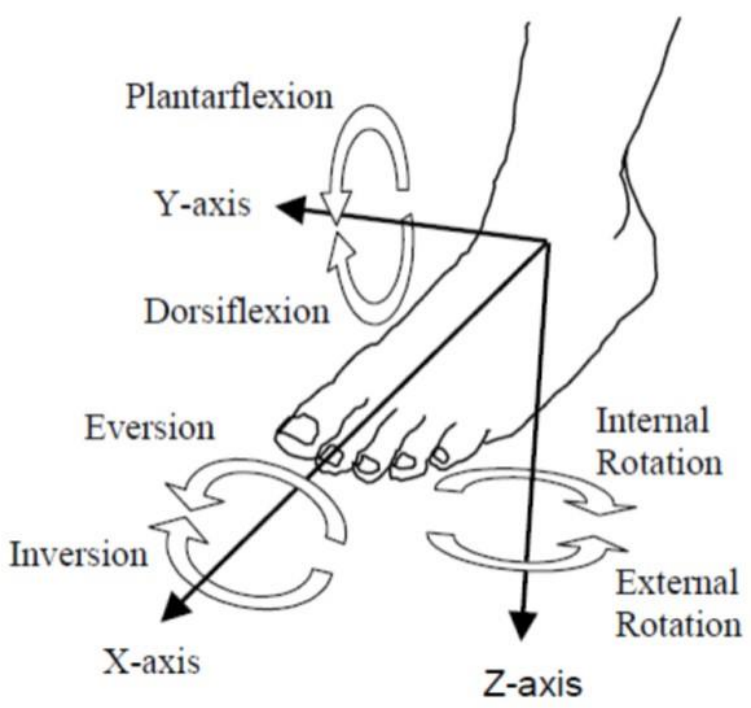

Figure 1: Gross rotations of the ankle permitted through the talocrural, subtalar, and syndesmosis joints (Mane, 2016; Shin et al., 2012). 
The foot and ankle is among the most frequently injured regions of the lower extremity in all levels of athletics (Barker et al., 1997; Hintermann, 1999), with lateral and syndesmotic ankle sprains accounting for approximately 85 to $90 \%$ of those injuries (Mane, 2016; Rubin and Sallis, 1996). Commonly in athletics, injuries to the ankle joint occur to the lateral structures due to severe inversion of the foot (Anderson et al., 2010; Golanó et al., 2010; Kaumeyer and Malone, 1980; Wolfe et al., 2001). The lateral structures (Figure 2) of the anterior talofibular ligament (ATaFL), calcaneofibular ligament (CFL), and posterior talofibular ligament (PTaFL), collectively the lateral collateral ligaments, constrain the calcaneus and talus from displacing relative to the fibula, thus preventing excessive inversion of the subtalar joint (Golanó et al., 2010; Kaumeyer and Malone, 1980; Norkus and Floyd, 2001).

Less common are injuries to the tibiofibular syndesmosis, occurring in less than $20 \%$ of all ankle sprains (Anderson et al., 2010; Bloemers and Bakker, 2006; Boytim et al., 1991; Gerber et al., 1998; Hopkinson et al., 1990; Lin et al., 2006; McCollum et al., 2012; Norkus and Floyd, 2001; Rammelt et al., 2008; Rose et al., 2002; Williams et al., 2007). Distally, the tibiofibular syndesmosis joint (Figure 2) is constrained by the anterior and posterior tibiofibular ligaments (ATiFL and PTiFL) and interosseous membrane (IOM). These ligaments prevent excessive motions of the fibula relative to the tibia and stabilize the leg during weight-bearing by transmitting forces from the tibia to the fibula (Norkus and Floyd, 2001; Skraba and Greenwald, 1984).

Concomitant to lateral and syndesmotic injuries are injuries to the deltoid ligament complex (Boytim et al., 1991; Campbell et al., 2014; Guise, 1976; McCollum et al., 2012; Teramoto et al., 2008; Williams et al., 2007). Positioned on the medial aspects of the medial malleolus, talus, and calcaneus, the deltoid ligament complex is often characterized with two main layers: superficial and deep (Campbell et al., 2014; Golanó et al., 2010; Kaumeyer and Malone, 1980; McCollum et al., 2012). The superficial deltoid complex (Figure 2) consists mainly of the anterior aspects of the fan-like deltoid ligament complex, termed the tibionavicular and tibiospring ligaments, spanning from the anterior medial malleolus to the navicular, talus, and calcaneus (Campbell et al., 2014; Norkus and Floyd, 2001). Inferior and posterior to the superficial deltoid complex is the deep deltoid complex (Figure 2), described as having two components branching from the tibia to 
the talus and to the calcaneus. These branches are termed as the tibiotalar and tibiocalcaneal ligaments (Campbell et al., 2014; Golanó et al., 2010; Kaumeyer and Malone, 1980). The deltoid complex is considered the strongest of the ankle ligaments and resists excessive subtalar eversion and talar external rotation (Norkus and Floyd, 2001).
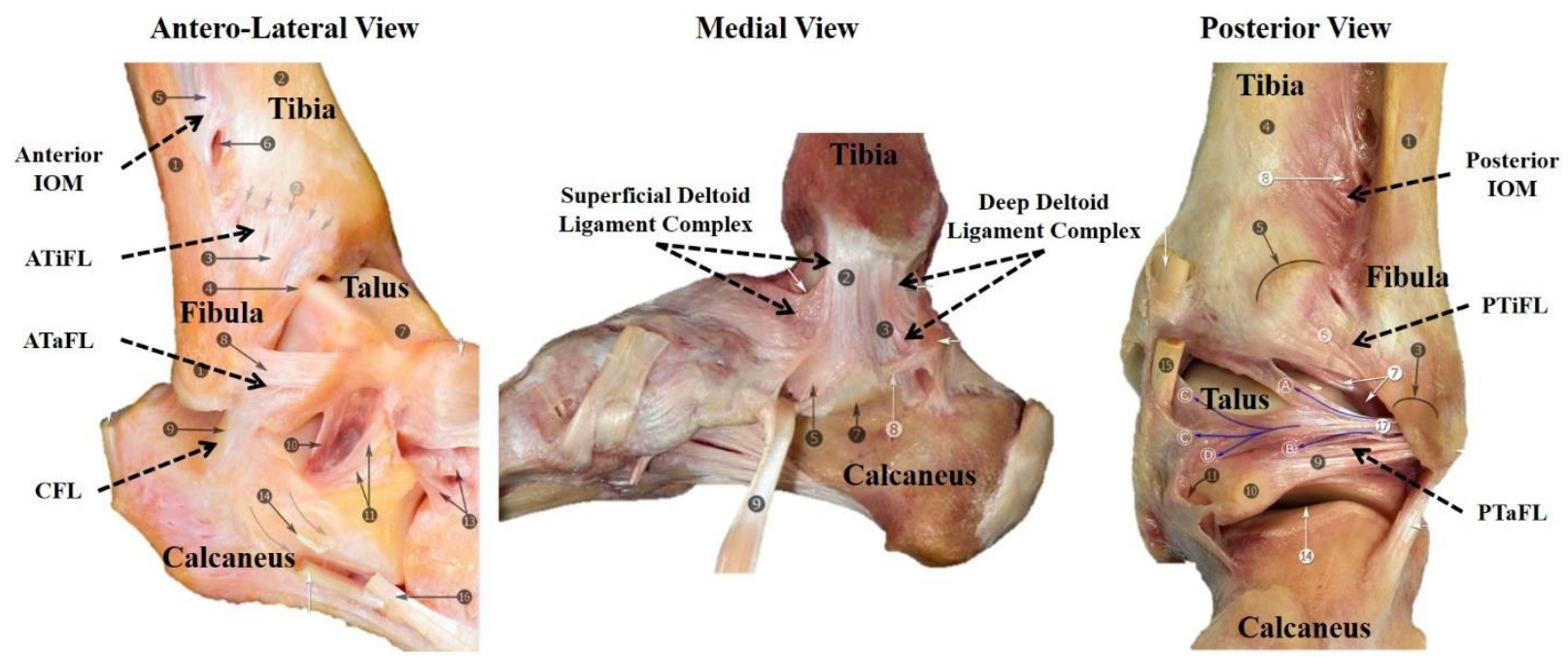

Figure 2: Osteoligamentous ankle anatomy with pertinent bones and ligaments labeled in the Antero-Lateral (left), Medial (middle), and Posterior (right) views: adapted from Golano et al. (2010).

Despite a lower occurrence rate compared to lateral ankle sprains, syndesmotic ('high') ankle sprains are accompanied by a lengthy recovery and rehabilitation period (Anderson et al., 2010; Boytim et al., 1991; Guise, 1976; Jones and Amendola, 2007; Lin et al., 2006; Norkus and Floyd, 2001; Teramoto et al., 2008; Williams et al., 2007). Syndesmotic ankle sprains are often attributed to one or all of the following concomitant injuries: tears and/or avulsions of the ATiFL, PTiFL, and IOM or fibula fractures (e.g., Danis-Weber B and C classified fractures) (Beumer, 2007; Dias and Foerster, 1974; Funk, 2011; Hopkinson et al., 1990; Hughes et al., 1979; Lauge-Hansen, 1950; Lin et al., 2006; McCollum et al., 2012; Williams et al., 2007; Wolfe et al., 2001).

American football is a widely cited contact sport for syndesmotic ankle injury, but these injuries are also common in sports such as ice hockey, skiing, lacrosse, and soccer (Bloemers and Bakker, 2006; Boytim et al., 1991; Fritschy, 1989; Lin et al., 2006; Rammelt et al., 2008; Teramoto et al., 2008; Wright et al., 2004). Over a five-year span, professional American football players had an 
average, per player, time loss of 2.5 weeks, 11.7 practices, and 1.4 games from syndesmotic ankle sprains compared to 1.25 weeks, 3.5 practices, and 0.3 games from lateral ankle sprains (Guise, 1976; Williams et al., 2007). Recovery time for syndesmotic ankle sprains can exceed 31 days and even up to 55 days, almost twice the recovery time compared to third-degree lateral ankle sprains (Hopkinson et al., 1990; Lin et al., 2006). These injuries are highly debilitating, where athletes have trouble performing moves (e.g., cutting and pushing off) and experience chronic pain (Anderson et al., 2010; Kaumeyer and Malone, 1980; Norkus and Floyd, 2001; Ogilvie-Harris et al., 1994; Taylor et al., 1992; Teramoto et al., 2008; Williams et al., 2007). Proper diagnoses and treatments of syndesmotic ankle sprains are also challenging (Jones and Amendola, 2007; Williams et al., 2007). With such damaging consequences and extended recovery time, understanding syndesmotic ankle sprain mechanics is paramount to inform future clinical care and prevention as well as injury mitigation techniques.

\subsubsection{Injury Mechanisms}

External foot rotation is hypothesized as the key mechanism of syndesmotic ankle injury (Anderson et al., 2010; Bloemers and Bakker, 2006; Guise, 1976; Lin et al., 2006; Norkus and Floyd, 2001; Teramoto et al., 2008; Waterman et al., 2010; Wei et al., 2010, 2012b; Williams et

al., 2007; Wolfe et al., 2001). Typically on the playing field (Figure 3), lateral blows to the leg cause the tibia to rotate internally as players are either lying prone on the ground with contact made to their heel, thus forcing the foot to the ground, or the foot is planted and the body rotates opposite of the foot (Guise, 1976; Norkus and Floyd, 2001). Hyper-dorsiflexion and hyper-eversion of the ankle have also been proposed as contributors to syndesmotic ankle sprains (Funk, 2011; Lin et al., 2006; Wei et al., 2010, 2012b; Williams et al., 2007). 

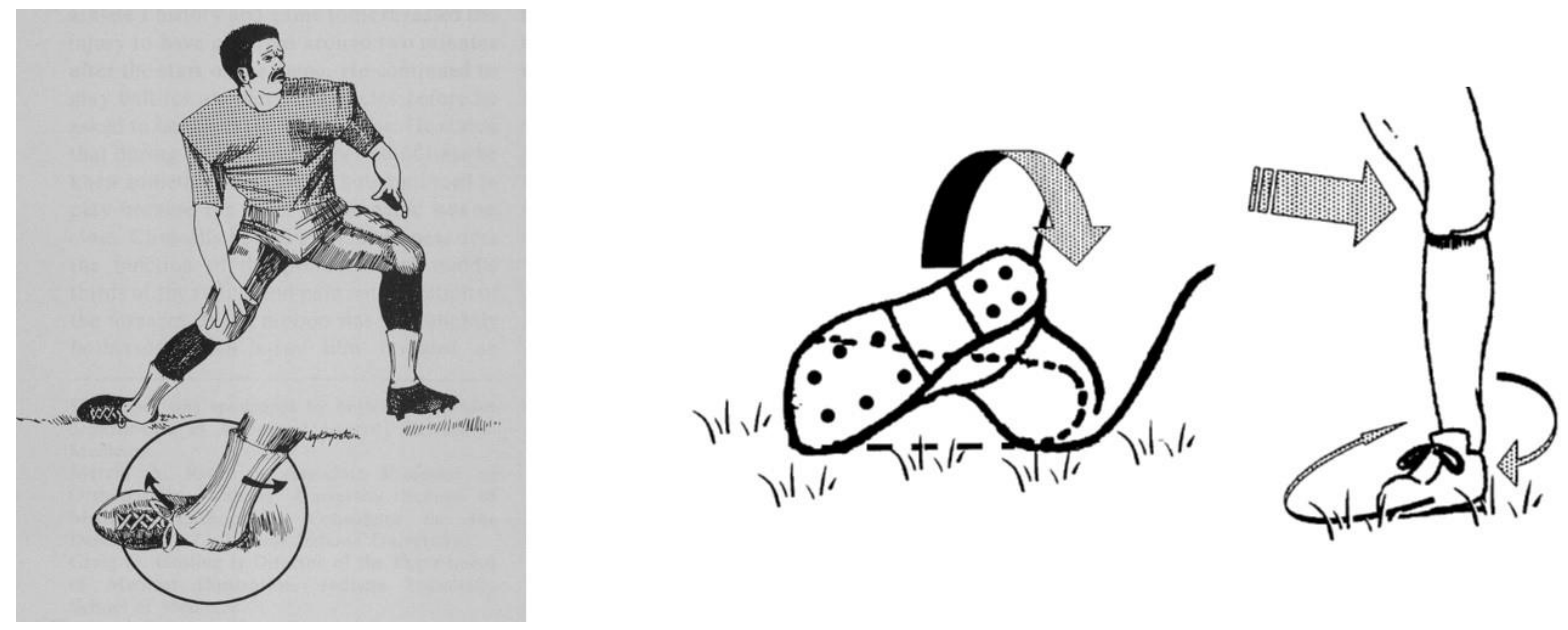

Figure 3: Hypothesized mechanism of injury on the playing field for American Football players where a lateral blow to the leg causes internal tibial rotation over a planted foot or when a player is lying prone on the ground (Left: Guise, 1976; Right: Norkus and Floyd, 2001).

External foot rotation is believed to cause the talus to wedge between the tibia and fibula articular surfaces, which generates tibiofibular diastasis by forcefully displacing the fibula laterally and posteriorly (Bonnin, 1970; Edwards and DeLee, 1984; Lin et al., 2006; Rose et al., 2002; Williams et al., 2007). This diastasis increases the risk of rupture to the ATiFL and PTiFL with possible propagation into the IOM (Anderson et al., 2010; Bloemers and Bakker, 2006; Boytim et al., 1991; Funk, 2011; Lin et al., 2006; Ogilvie-Harris et al., 1994; Wolfe et al., 2001). However, tibiofibular diastasis is a subtle kinematic phenomenon, and is indicated by tibiofibular clear space, normally less than $6 \mathrm{~mm}$ wide (Dattani et al., 2008; Harper and Keller, 1989), increasing by 1 to $6 \mathrm{~mm}$ during syndesmotic injury compared to the uninjured (Bonnin, 1970; Husfeldt, 1937; Rammelt et al., 2008; Sclafani, 1985; Williams et al., 2007). Even further, diastasis amounted to only $2.3 \mathrm{~mm}$ after sectioning of the ATiFL (Xenos et al., 1995). Syndesmotic injury can even be present without the presence of frank diastasis shown in stress radiographs (Edwards and DeLee, 1984; Nussbaum et al., 2001); only after a complete clinical squeeze or external rotation stress test is syndesmotic injury properly diagnosed (Nussbaum et al., 2001). Therefore, with such a subtle phenomenon driving possible debilitating ligamentous injuries, experimental investigations of syndesmotic injuries must allow for natural tibiofibular diastasis. 


\subsubsection{Previous Experimental Efforts}

Previous studies executed experiments to re-create syndesmotic ankle sprains in cadaveric limbs (Lauge-Hansen, 1950; Markolf et al., 1989; Michelson et al., 1997; Wei et al., 2010, 2012b; Xenos et al., 1995). These studies provided a basis for the current investigations regarding syndesmotic ankle sprains; however, several deficiencies (Table 1) were found and needed to be addressed. Older, smaller cadavers were tested (Markolf et al., 1989; Michelson et al., 1997) which resulted in a high occurrence of fibula fracture at failure instead of ligamentous injury (Table 1) compared to studies testing younger cadavers (Villwock et al., 2009; Wei et al., 2010, 2012b). Presumably, bone deterioration in the elderly cadavers contributed to the high occurrence of fibula fracture rather than ligamentous rupture.

Table 1: Previous ankle rotation studies with mined data for specimen age, vertical preload, measured torque, and injuries at failure. Experimental deficiencies for each study are detailed.

\begin{tabular}{c|c|c|c|l|l}
\hline Study & $\begin{array}{c}\text { Age } \\
\text { Range of } \\
\text { Specimens } \\
\text { (yr.) }\end{array}$ & $\begin{array}{c}\text { Vertical } \\
\text { Preload } \\
\text { (N) }\end{array}$ & $\begin{array}{c}\text { Peak } \\
\text { Measured } \\
\text { Torque } \\
\text { (N-m) }\end{array}$ & Injuries at Failure & $\begin{array}{c}\text { Experimental } \\
\text { Deficiencies }\end{array}$ \\
\hline $\begin{array}{c}\text { Markolf et } \\
\text { al., 1989 }\end{array}$ & $\begin{array}{c}\text { Young and } \\
\text { Old }\end{array}$ & 0 & $45.3 \pm 18.7$ & $\begin{array}{l}\text { 12 Fibula fx; } \\
\text { 5 Lateral ligament } \\
\text { injuries }\end{array}$ & $\begin{array}{l}\text { Tibia and Fibula } \\
\text { rigid; } \\
\text { Rigid foot }\end{array}$ \\
\hline $\begin{array}{c}\text { Michelson } \\
\text { et al., 1997 }\end{array}$ & 60 to 80 & 700 & 10 to 40 & $\begin{array}{l}\text { 15 of 30 Fibula fx; } \\
\text { 7 of 30 ATiFL } \\
\text { injuries; } \\
\text { 5 of 30 Deltoid } \\
\text { ligament injuries; } \\
\text { 10 of 30 Lateral } \\
\text { ligament injuries }\end{array}$ & $\begin{array}{l}\text { Tibia and Fibula } \\
\text { rigid; } \\
\text { Foot clamped }\end{array}$ \\
$\begin{array}{c}\text { Villwock et } \\
\text { al., 2009 }\end{array}$ & $40 \pm 11$ & $\sim 2000$ & $\begin{array}{l}70 \pm 12 \\
\text { (potted); } \\
78 \pm 18 \\
\text { (taped) }\end{array}$ & $\begin{array}{l}\text { 5 Fibula fx; } \\
\text { 5 PTaFL injuries; } \\
\text { 4 Superficial } \\
\text { deltoid injury }\end{array}$ & $\begin{array}{l}\text { Tibia and Fibula } \\
\text { rigidly coupled; } \\
\text { Potted and taped } \\
\text { feet }\end{array}$ \\
\hline $\begin{array}{c}\text { Wei et al., } \\
2010\end{array}$ & $43 \pm 17$ & 2000 & $69.5 \pm 11.7$ & $\begin{array}{l}\text { 1 Fibula fx; } \\
\text { 5 PTia fx; } \\
\text { 1 Superficial } \\
\text { deltoid injury }\end{array}$ & $\begin{array}{l}\text { Tibia and Fibula } \\
\text { rigidly coupled; } \\
\text { Potted foot }\end{array}$ \\
\hline $\begin{array}{c}\text { Wei et al., } \\
\text { 2012b }\end{array}$ & $56 \pm 12$ & 1500 & $84.4 \pm 16.9$ & $\begin{array}{l}\text { 1 Fibula fx; } \\
\text { 5 Deltoid ligament } \\
\text { injuries; } \\
\text { 1 ATiFL injury }\end{array}$ & $\begin{array}{l}\text { Tibia and Fibula } \\
\text { rigidly coupled; } \\
\text { Taped foot }\end{array}$ \\
\hline
\end{tabular}


Other studies artificially restricted the motion of the leg (Table 1) during testing by rigidly fixing the fibula to the tibia or not permitting natural foot translation (Markolf et al., 1989; Michelson et al., 1997; Villwock et al., 2009; Wei et al., 2010, 2012b; Xenos et al., 1995). Interactions between the tibia and fibula play an important role in syndesmotic ankle sprain causation, particularly through tibiofibular diastasis (Lin et al., 2006; Teramoto et al., 2008; Xenos et al., 1995). By rigidly coupling the tibia and fibula, this natural tibiofibular kinematic interaction is lost. Some experiments (Markolf et al., 1989; Wei et al., 2010, 2012b; Xenos et al., 1995) prevented the foot from translating or the arch of the foot from flattening, thus hindering natural interactions of all ankle and foot bones and subsequent ligament recruitment during rotation within the ankle. In natural joint motion, external and internal rotation of the foot occurs about a moving axis which is determined by the interactions between the tibia, fibula, talus, and calcaneus (Funk, 2011; Rastegar

et al., 1980). Restricting this moving axis to an artificial, fixed axis of rotation has the potential to result in altered ankle mechanics (Skraba and Greenwald, 1984) and, in turn, produce ankle bone kinematics and injury patterns that are not representative of what would occur naturally during excessive foot rotation.

\subsection{Research Objectives}

The overall objective of this thesis was to determine how syndesmotic ankle sprains are caused, subject to the hypothesized injury mechanism (external foot rotation). Various initial ankle postures were investigated to determine their effects on syndesmotic ankle sprain causation. Specifically, this thesis aimed to accurately and realistically re-create ligamentous syndesmotic ankle sprains in cadavers. Significant efforts were made to improve the boundary conditions enacted on specimens during applied foot rotation experiments from those of previous studies. The boundary condition improvements permitted the realistic re-creation of gross leg motion that typically induces syndesmotic ligamentous injury in vivo. With the improved experimental boundaries, the resulting bony motion within the cadaveric leg was measured as external foot rotation was applied. This measured bony motion was then related to the incidence of injuries within the ankle. A complete list of objectives follows: 
Objective 1: Improve experimental boundary conditions for which ankle mechanical responses to applied foot rotation are measured.

Objective 1A: Perform proof-of-concept non-injurious experiments with improved boundary conditions and implement proper mechanical response (e.g., kinematic) measurement techniques.

Objective 1B: Implement a coordinate system defined with bone geometry and anatomical landmarks which can quantify relative bone kinematics in the foot and ankle and is interpretable for clinical definitions of ankle rotations.

Objective 2: Characterize syndesmotic ankle sprains in non-senescent, sizable male cadaver legs oriented in an initially neutral ankle flexion posture.

Objective 2A: Confirm the hypothesized injury mechanism of external foot rotation.

Objective 2B: Accurately determine a consistent ankle injury sequence and propagation during forceful external foot rotation by utilizing multiple ankle mechanical response measurement techniques (i.e., kinetic, kinematic, strain, etc.).

Objective 2C: Determine a kinematics-based injury occurrence limit, relative to external foot rotation, for syndesmotic injury in an initially neutral ankle posture.

Objective 3: Describe effects of initial ankle flexion posture variations on syndesmotic injury incidence compared to the neutral posture.

Objective 3A: Determine the change in ankle injury propagation when subject to forceful external foot rotation in multiple ankle flexion postures.

Objective 3B: Determine the effects of ankle flexion posture on syndesmotic injury occurrence relative to external foot rotation.

Objective 3C: Determine clinical implications for syndesmotic ankle sprain diagnosis with the improved injury re-creation experiments.

Objective 4: Deliver accurate and reliable injury sequence and kinematic data for syndesmotic injury to be implemented in a finite element model.

Objective 4A: Characterize syndesmotic injury occurrence limits in multiple loading conditions to inform future countermeasure designs intending to mitigate syndesmotic ankle sprains. 


\subsection{Study Design}

Previous experiments investigating the effects of forceful external foot rotation implemented artificially-restrictive boundary conditions such that the tibia and fibula were rigidly coupled and natural foot motion was constrained. The experiments detailed in this thesis improved upon these boundary conditions by leaving the fibula unconstrained and permitting foot translation during applied foot rotation. Chapter 2 details the non-injurious proof-of-concept study completed to determine the viability of these new boundary conditions, not only for proper re-creation of ankle loading but also for accurate measurement of ankle mechanical responses during applied foot rotation (Objective 1).

Chapter 3 details the implementation of these new boundary conditions into an injurious, dynamic scenario. After the first iteration of injurious experiments, these boundary conditions were further improved to more accurately re-create a realistic syndesmotic injury scenario. From these injurious experiments a confirmation of injury mechanism and injury sequence was determined for the ankle during applied foot rotation (Objective 2).

Chapter 4 describes the data analysis completed to determine a kinematic injury occurrence interval limit for syndesmotic ankle sprains (Objective 2). Effects of initial ankle flexion posture on this injury interval are also discussed (Objective 3). Clinical implications of the improved experimental syndesmotic injury re-creation are conferred for possible improvements to future diagnosis and care techniques (Objective 3).

Chapter 5 describes the overall implications and conclusions of the experimental work detailed in this thesis. Applicability and limitations of the data presented in this thesis are discussed. Future uses of the data in this thesis are proposed including, improving the biofidelic response of computational ankle surrogate models and countermeasure designs aimed at mitigating syndesmotic ankle sprains (Objective 4). 


\subsubsection{Research Study Assumptions}

Before discussing the results and conclusions of this thesis, a discussion of the major assumptions utilized in the experimentation must be detailed. A kinematic predictor of syndesmotic injury was assumed, thus muscle forces were not considered during experimentation. Therefore, a nominal compressive load was applied to every specimen to simulate weight bearing during dynamic, injurious scenarios (Wei et al., 2012a, 2010). This was assumed sufficient to induce joint congruency and subsequent ligament recruitment during applied foot rotation. To limit inertial effects, legs were rotated at a rate lower than those experienced by athletes on-field. Sensitivity to the rate of rotation was not performed, therefore a dependence on rate of loading (rotation) and magnitude of loading (preload) was deemed negligible in this research.

With a predominantly ligamentous injury, and since ligaments constrain bony motion within joints, the experimental efforts were focused on reproducing proper leg and ankle bony motion, such that tibiofibular diastasis was induced. Ligament elongation can be inferred from this bony motion, and confirmed with injury dissection results, as the talus rotates in the intra-malleolar mortise and the fibula displaces from the tibia. External foot rotation and tibiofibular diastasis are widely cited in the literature as contributing factors to syndesmotic injury, however muscle force is not. Furthermore, reproducing muscle forces in cadaveric specimens is difficult. Though muscle forces, especially loads through the Achilles tendon, may play a role in the leg's mechanical response to external rotation on the athletic field, bone motion (kinematics) is the proposed determining factor for syndesmotic ankle sprains clinically. Therefore, it was assumed if the kinematic predictor was correct, then a proper injury occurrence limit for syndesmotic ankle sprains, regardless of muscle forces, could be ascertained. 


\section{Chapter 2: Proof-of-Concept-Quasi-Static Experiments}

\subsection{Introduction}

Forceful external foot rotation causes tibiofibular diastasis as the talus rotates within the intramalleolar mortise, forcing the distal fibula laterally and posteriorly from its articular surface the tibia (Boytim et al., 1991; Lin et al., 2006; Rose et al., 2002; Williams et al., 2007). This can injure structures in the distal tibiofibular syndesmosis, causing significant athletic time lost and clinical treatment challenges (Anderson et al., 2010; Bloemers and Bakker, 2006; Funk, 2011; Guise, 1976). Tibiofibular diastasis is a subtle kinematic phenomenon, such that tibiofibular clear space increases by as little as $6 \mathrm{~mm}$ (Williams et al., 2007). Previous experiments provide coordinate definitions to measure gross joint motion (Siegler et al., 1988; Wei et al., 2011; Wu et al., 2002). However, subtle bone-specific kinematic interactions (e.g., tibiofibular diastasis) are difficult to distinguish in these gross joint coordinate systems.

Changes in the three-dimensional orientation of bodies requires careful definition. Anatomical joints in the human body are often modeled as mathematical and mechanical joints to describe gross joint motion (Lundberg and Svensson, 1993). However, clinical professionals have their own definitions of joint motion in terms of gross anatomical motion (Gray, 1918; Houglum and Bertoti, 2012; Moore et al., 2010). Therefore, a fundamental part of biomechanics is to traverse the disparities between gross joint motion mathematical definitions and practical clinical terms common among those who examine, diagnose, and treat injuries in the human body (Cappozzo et al., 2005; Fioretti et al., 1997). However, different conventions have been used to describe joint motion. Cappozzo et al. (2005) compared these conventions, and concluded that the choice of convention was less important than the clear reporting of convention. However, Cappozzo et al. (2005) did admit that a geometric method (Allard et al., 1997; Paul, 1992) could be qualitatively close to clinical descriptions of joint motion.

Clinical definitions of the rotation (external/internal), flexion (dorsi-/plantar-) and version (e-/in-) angles of the human ankle describe the gross relative motions of the leg and foot in terms conducive to geometric interpretation (Gray, 1918; Houglum and Bertoti, 2012; Moore et al., 
2010). However, biomechanics studies favor an overall description of gross joint motion (e.g., Siegler et al., 1988; Wu et al., 2002), or Euler angles convention (e.g., de Asla et al., 2006; Yamaguchi et al., 2009). Lundberg et al. (1989) and Svensson et al. (1989) tracked motion of the tibia and fibula in vivo, however the six-degree-of-freedom bone-specific motion of all pertinent ankle bones has not been measured in cadaveric specimens during applied foot rotation. All of these previous coordinate system definitions informed the work detailed in this chapter, however coordinate systems that describe the relative motion of bones within the ankle are necessary to interpret measured bone kinematics in clinically relevant terms as well as infer ligament elongation between individual bones. Inferring ligament elongation between specific bones in the ankle is important for distinguishing ligament injuries within the ankle during applied foot rotation.

Though defining coordinate systems which are technically rigorous yet practical for clinical interpretations is important to fully understand the effects of applied foot rotation on ankle bone kinematic responses, these kinematics cannot be considered reliable without the use of proper experimental boundary conditions. These boundary conditions must not artificially restrict gross joint motion yet still permit relative motion of bones, e.g. tibiofibular diastasis, to properly recreate a potential syndesmotic injury scenario. Previous experiments investigated the effects of foot rotation on leg mechanics and the syndesmosis joint (Markolf et al., 1989; Michelson et al., 1997; Villwock et al., 2009; Wei et al., 2012b, 2010; Xenos et al., 1995), but with limitations (Table 1). Markolf et al. (1989), Michelson et al. (1997), Villwock et al. (2009), and Wei et al. (2012b, 2010) disarticulated the tibia and fibula mid-shaft, thus disrupting the proximal interosseous membrane (IOM) and tibiofibular joint. These studies rigidly fixed the tibia and fibula together, preventing the fibula from moving freely during rotation, therefore altering ankle mechanics (Skraba and Greenwald, 1984). These studies also restricted foot translation by rigidly fixing or taping the foot (Table 1). Though Xenos et al. (1995) allowed the fibula to move freely, the leg was rigidly constrained in multiple locations and foot translation was not permitted. This created an unclear axis of rotation in the ankle by preventing the foot from translating naturally during foot rotation. These boundary conditions artificially constrained fibula and foot motion which prevents natural interactions among ankle anatomical structures and restricts accurate recreation of foot rotation about the ankle's moving axis (Funk, 2011; Rastegar et al., 1980). 
Geometric coordinate system conventions proposed previously (Allard et al., 1997; Cappozzo et al., 2005; Paul, 1992) mainly focused on human locomotion in the knee joint, with one for the ankle (Green et al., 2011). Therefore, the main objective of this chapter was to adapt these geometric conventions for the ankle joint to quantify relative rigid-body (bone-specific) kinematics in cadaveric legs during applied foot rotation (Objective 1). Another objective of this chapter was to impose functionally relevant boundary conditions on the leg (Objective 1), such that a more realistic anatomic configuration is created where the fibula is unconstrained and foot translation is permitted (Mait et al., 2015, 2017b). Feasibility and applicability of the geometry-based coordinate system definition and functionally relevant boundary conditions were determined in a small-scale, proof-of-concept study on a small sample of cadaveric legs in a non-injurious, repeated trial experimental protocol. With imposing boundary conditions designed to re-create a more realistic loading within the leg, reliable kinematic responses to applied foot rotation can be measured and will be used to inform future experiments focused on describing ankle syndesmosis injury characteristics.

\subsection{Methods}

\subsubsection{Specimen Preparation}

Experiments were conducted on fresh-frozen left lower limbs from three male cadavers (Table 2). The specimens were acquired with the approval of and prepared in accordance with the policies and procedures of the UVA Center for Applied Biomechanics Oversight Committee (Ethics Approval \#: CAB2014-07). All specimens were confirmed free of infectious diseases, including $\mathrm{HIV}$ and Hepatitis $\mathrm{B} / \mathrm{C}$, stored at $-15^{\circ} \mathrm{C}$, and thawed at room temperature for 48 hours prior to test preparation.

Table 2: Whole-body specimen anthropometry (gender, age at time of death, height, and weight) for the three specimens tested in the small-scale, proof-of-concept study.

\begin{tabular}{ccccc}
\hline Specimen ID\# & Gender & Age (yr.) & Height (cm) & Weight (kg) \\
\hline 616L & Male & 46 & 177 & 113 \\
739L & Male & 47 & 183 & 107 \\
743L & Male & 31 & 188 & 100 \\
\hline
\end{tabular}


Specimens were disarticulated at the knee and soft tissue surrounding the tibial plateau was removed. Soft tissue was also removed from the anterior, medial, and posterior aspects of the tibia shaft from the tibial plateau to approximately $10 \mathrm{~cm}$ distal. However, soft tissue surrounding the fibula was left intact. All tibiofibular ligaments and interosseous membrane (distal and proximal) were left intact (Mait et al., 2015, 2017b).

The proximal tibia was rigidly attached to a potting cup using a set of wood screws across the top of the tibial plateau and along the anterior, medial, and posterior aspects (at varying heights and angles) of the proximal tibia shaft (Figure 4). To complete the rigid connection between proximal tibia and potting cup, Bondo body filler (part \#261, 3M Company, St. Paul, MN, USA) was applied to fill all gaps between the tibial plateau and potting cup top. Therefore, the proximal tibia was rigidly fixed but the fibula was unconstrained (Mait et al., 2015, 2017b).
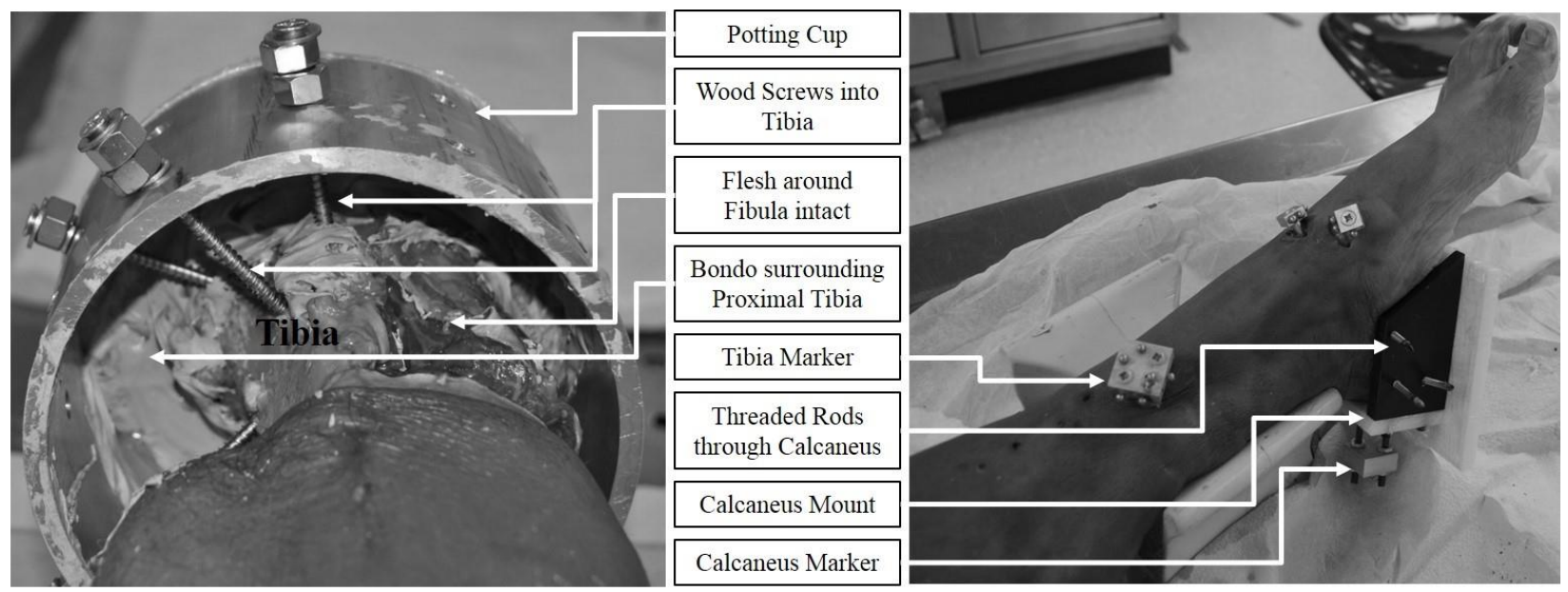

Figure 4: Potted tibia of a specimen where wood screws are driven into the proximal shaft of the tibia and with Bondo body filler covering the anterior, posterior, and medial sides of the proximal tibia (left). Placement of the tibia and calcaneus external motion-tracking marker cubes as well as the open-faced mount around the calcaneus with threaded rods driven from lateral to medial (right). Several holes were placed on the lateral face of the calcaneus mount for threaded rods to pass through to accommodate different sizes of specimens, and threaded rods anchored into the medial face made of solid polyvinyl chloride.

Aluminum external motion-tracking marker cubes were affixed to the tibia, fibula, talus, and navicular via transdermal screws passing through standoffs (Figure 5). The insertion paths of these screws were designed for minimal disruption of surrounding soft tissue. A mount assembled with 
acetal homopolymer resin and polyvinyl chloride, reinforced with machine screws, was fixed to the calcaneus using threaded rods passing from lateral to medial directly tapping into the polyvinyl chloride (Figure 4). A motion-tracking marker cube was fixed to the posterior face of this mount (Mait et al., 2017b). Placement of these markers was confirmed (Figure 5) with static X-rays (Model\#: EPX-F2400, ECOTRON, Seoul, Korea). Collimated spiral computed tomography (CT) scans (slice resolution $=0.625 \mathrm{~mm} ; \mathrm{FOV}=45 \mathrm{~cm}$; in-plane resolution $=0.9 \mathrm{~mm} \times 0.9 \mathrm{~mm}$ ) of each specimen were taken before testing to confirm absence of bony trauma and measure the positions of the motion-tracking markers relative to each bone (Mait et al., 2015). For the CT scans, specimens were placed in a radio-opaque apparatus that held the legs in a neutral posture (Figure 5), defined such that the first phalanx, approximate calcaneus centroid, and tibia long-axis formed a right angle in the sagittal plane.
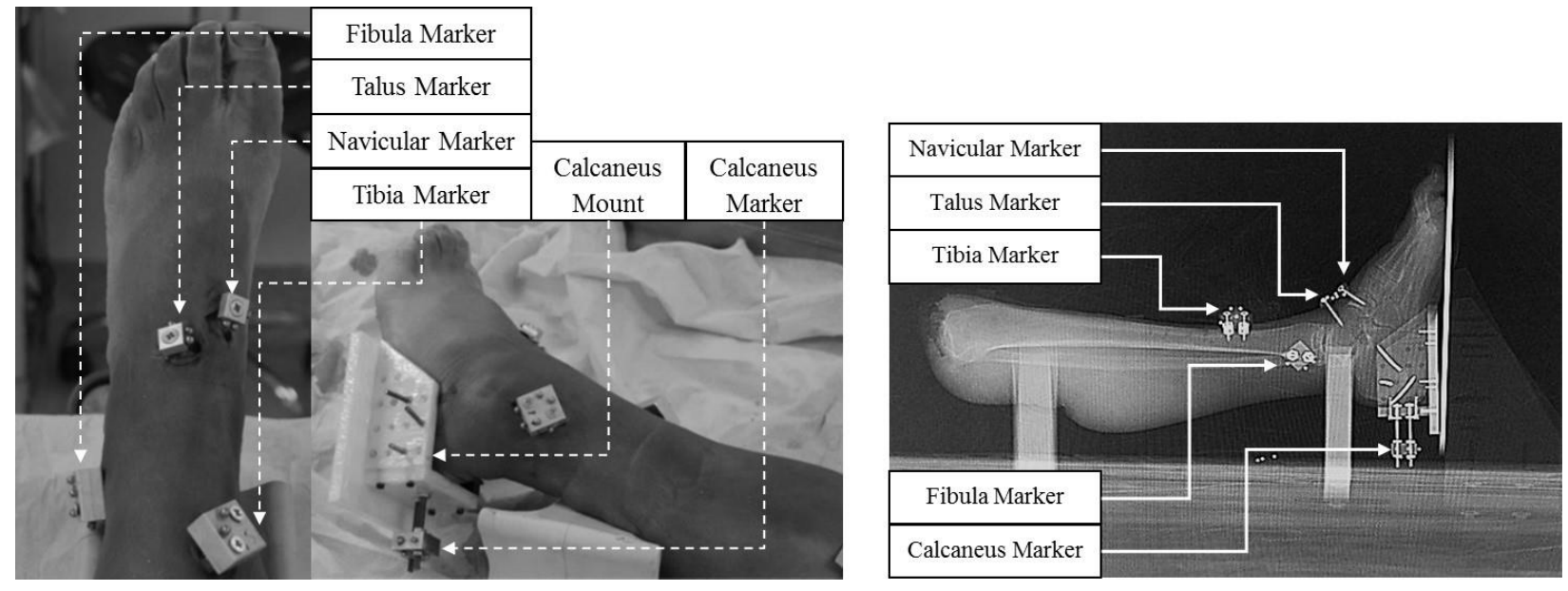

Figure 5: External motion-tracking marker cube placement in the tibia, fibula, navicular, and talus bones as well as the calcaneus mount (left). X-ray image of marker cube placement confirmation (right).

\subsubsection{Coordinate System Definitions}

Clinical studies (Gray, 1918; Houglum and Bertoti, 2012; Moore et al., 2010) describe ankle joint rotations as motion of the foot relative to the leg (i.e., tibia) within the planes of the body (Table 3). External/internal rotation of the ankle is described as rotation of the foot relative to the leg's long-axis in the transverse plane. Dorsi/plantarflexion of the ankle is described as rotation of the foot toward/away from the leg's long-axis in the sagittal plane which contains both the foot and leg's long-axis. Finally, e/inversion of the ankle is described as rotation of the leg's long-axis relative to the foot's long-axis in the coronal plane. To relate these clinical definitions of ankle 
rotations, a coordinate system that relates the motion of the leg's long-axis to the foot's long-axis must be defined. A coordinate system defined locally on the tibia and calcaneus and described with geometric bone features on each specimen was chosen (Table 3). Though the ankle joint is comprised of three separate joints (subtalar, talocrural, and syndesmosis; Chapter 1), the subtalar and talocrural joints act as one, on the whole through ligamentous constraints, to permit ankle rotations such as e/inversion, dorsi/plantarflexion, and external/internal rotation (Nigg et al., 1990). Independent physiologic motions between the talocrural and subtalar joints do occur (Roach et al., 2016) through these ligamentous constrains, however these two joints combine to permit most of the ankle e/inversion and dorsi/plantarflexion. Therefore, if calcaneus motion is prescribed experimentally, the ligamentous constraints between the talus and calcaneus will force the talocrural joint to follow the input motion in the subtalar joint. Thus, motion of the foot was assumed to be synonymous with motion of the calcaneus in the geometrically defined coordinate system (Table 3). This allows for the description of relative motion among ankle bones to be interpreted in clinical terms for ankle e/inversion, dosi/plantarflexion, and external/internal rotation.

Table 3: Definitions of ankle rotation, flexion, and version angles: clinical vs. geometric (based on tibia and calcaneus local coordinate systems).

\begin{tabular}{c|c|c}
\hline Angle & $\begin{array}{c}\text { Clinical Definition } \\
\text { (Gray, 1918; Houglum \& } \\
\text { Bertoti, 2012; Moore et al. 2010) }\end{array}$ & $\begin{array}{c}\text { Proposed Local Coordinate System } \\
\text { Definition (Geometric) }\end{array}$ \\
\hline $\begin{array}{c}\text { Rotation } \\
\text { (external +, } \\
\text { internal -) }\end{array}$ & $\begin{array}{c}\text { Rotation of the foot about the leg's } \\
\text { long axis }\end{array}$ & $\begin{array}{c}\text { Angle in the tibia's x-y plane between the } \\
\text { tibia's x axis and the projection of the } \\
\text { calcaneus'x axis on the tibia's x-y plane }\end{array}$ \\
$\begin{array}{c}\text { Flexion } \\
\text { (dorsiflexion +, } \\
\text { plantarflexion -) }\end{array}$ & $\begin{array}{c}\text { Rotation of the foot toward/away } \\
\text { from the leg in a plane containing } \\
\text { both the leg and the foot }\end{array}$ & $\begin{array}{c}\text { Angle in the calcaneus' z-x plane between } \\
\text { the calcaneus' z axis and the projection of } \\
\text { the tibia's z axis on the calcaneus' z-x plane }\end{array}$ \\
\hline $\begin{array}{c}\text { Version } \\
\text { (eversion }+, \\
\text { inversion -) }\end{array}$ & $\begin{array}{c}\text { Rotation of the leg about the foot's } \\
\text { long axis }\end{array}$ & $\begin{array}{c}\text { Angle in the calcaneus'y-z plane between } \\
\text { the calcaneus' z axis and the projection of } \\
\text { the tibia's z axis on the calcaneus'y-z plane }\end{array}$ \\
\hline
\end{tabular}

Vectors can be expressed in multiple coordinate systems where the coordinate system is first defined by specifying the location of the origin and then the direction of three mutually orthogonal axes with corresponding unit vectors (Bate et al., 1971; Shaw et al., 2009). These vectors can then be expressed in any reference frame by transforming the vector components to the desired 
reference frame. To adhere to the clinical interpretations of ankle joint motion described in Table 3 , this desired reference frame was chosen as the local coordinate system of the tibia. This was defined intentionally since experimental inputs of gross leg motion (i.e., applied foot rotation and compressive preload) were designed to act through the fixed proximal tibia. The resultant motion of all other ankle and foot bones react to this input tibia motion. Therefore, relative motion of the foot and ankle bones were expressed relative to the tibia coordinate system $\left(\mathrm{CS}_{\mathrm{Tibia}}\right)$.

Now to define this local tibia coordinate system, geometry of the bone and anatomical landmarks (Camacho et al., 2002; Cappozzo et al., 2005; Fioretti et al., 1997; Green et al., 2011) were utilized. All bone geometry and anatomical landmarks were digitized in Mimics 18.0 (Materialise NV, Leuven, Belgium) using the pre-test CT scans in the neutral ankle posture. Inertial axes of the tibia were defined automatically in Mimics 18.0. The long-axis of the tibia (i.e., leg) was defined as the axis about which the mass moment of inertia of the tibia was least, assuming homogenous density. The long-axis was then defined as the local z-axis, positive inferiorly (Figure 6, top). The origin of $\mathrm{CS}_{\text {Tibia }}$ was then defined where the z-axis and exterior surface of the tibial plafond intersected (Figure 6, top-middle). A plane was formed by the z-axis and a line ( $\left.\mathrm{y}^{\prime}\right)$ drawn between the anterior-inferior tip of the medial malleolus (chosen by the experimenter) and the origin (Figure $\mathbf{6}$, middle). The local $\mathrm{x}$-axis was defined as perpendicular to this plane containing the $\mathrm{z}$-axis and $y^{\prime}$ and was positive anteriorly (Figure 6, bottom-middle). Finally, the y-axis was defined as perpendicular (cross-product of vectors) to the $\mathrm{x}$ - and $\mathrm{z}$-axes and passed through the origin (Figure 6, bottom), positive medially for left legs and positive laterally for right legs (Mane, 2016). 

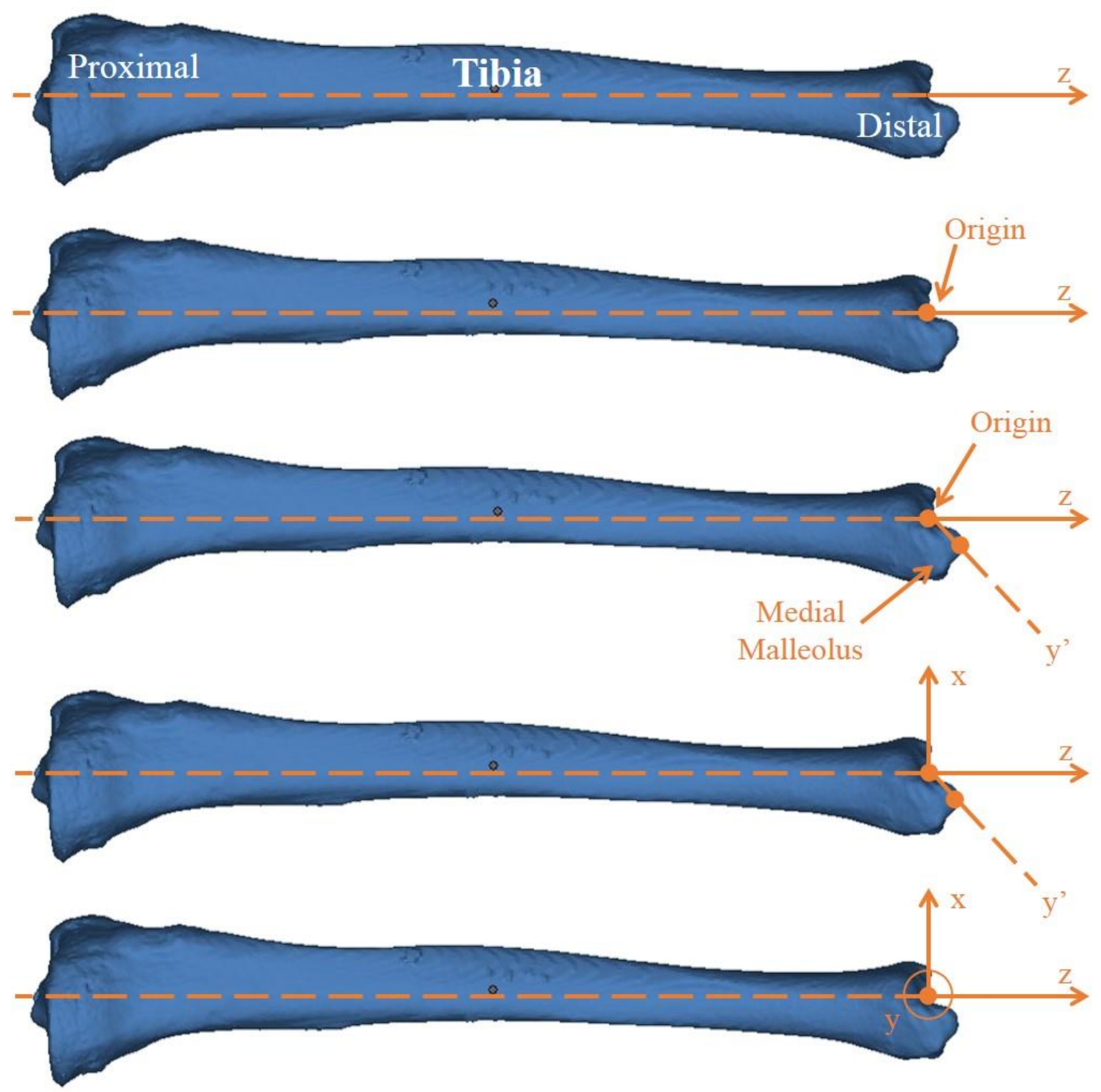

Figure 6: Method to define the local coordinate system of the tibia (left leg) using geometry of the bone (inertial axes) and anatomical landmarks (plafond and medial malleolus).

For the fibula, the origin was defined at the centroid of the lateral malleolus. The lateral malleolus was defined using the most postero-medial eminence of the distal fibula (i.e., closest to the lateraldistal tibia), which was chosen by the experimenter (Figure 7, left). A plane perpendicular to the fibula local z-axis (defined with Mimics 18.0) and containing the postero-medial eminence landmark was defined and separated the lateral malleolus from the rest of the fibula shaft. From 
this, the origin of the fibula was defined at the centroid of the lateral malleolus (Figure 7, right). The local axes for the fibula ( $\mathrm{x}, \mathrm{y}$, and $\mathrm{z}$ ), with origin in the lateral malleolus, were then defined as parallel to those in the $\mathrm{CS}_{\text {Tibia }}$ (Mane, 2016) in a neutral ankle posture.
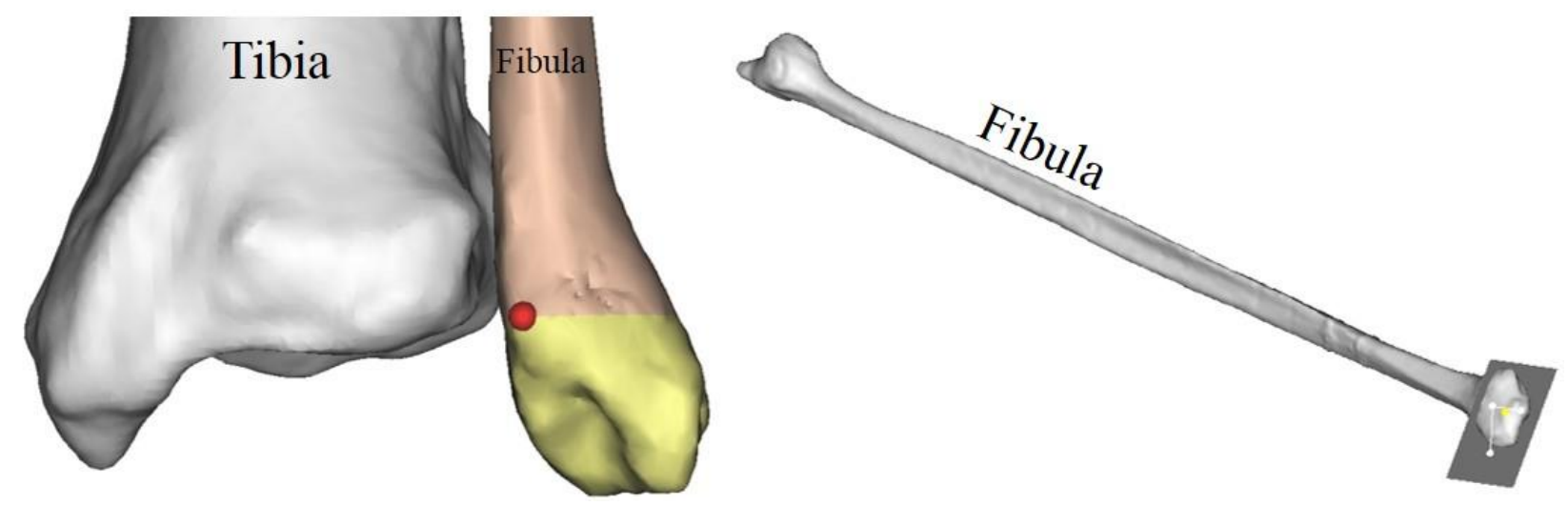

Figure 7: Method to define the origin of the fibula (right leg) geometrically in Mimics 18.0. Left: eminence on the fibula most posterior and medial (i.e., closest to lateral-distal tibia), denoted as a red dot, with defined lateral malleolus in yellow. Right: indication of origin in lateral malleolus (yellow dot) with plane denoting the slice taken from fibula shaft to demark lateral malleolus.

For any other ankle or foot bones (i.e., talus, calcaneus, navicular, and metatarsals), the origin of their respective coordinate systems were defined at the bone's centroid. All centroids were digitized automatically by Mimics 18.0. The axes (x, y, and z) of each bone's local coordinate system were defined as parallel to those of the $\mathrm{CS}_{\text {Tibia }}$ in the neutral ankle posture (Figure 8). Therefore, for each specimen, the tibia local coordinate system was defined based on tibia anatomical landmarks and geometry and all other bone local coordinate systems were defined parallel to that on the tibia with the bone's origin defined geometrically at the respective centroids. From these local bone coordinate system definitions, each bone's motion can be transformed into $\mathrm{CS}_{\text {Tibia }}$ to describe the angular and linear displacements of the bones relative to the tibia. 


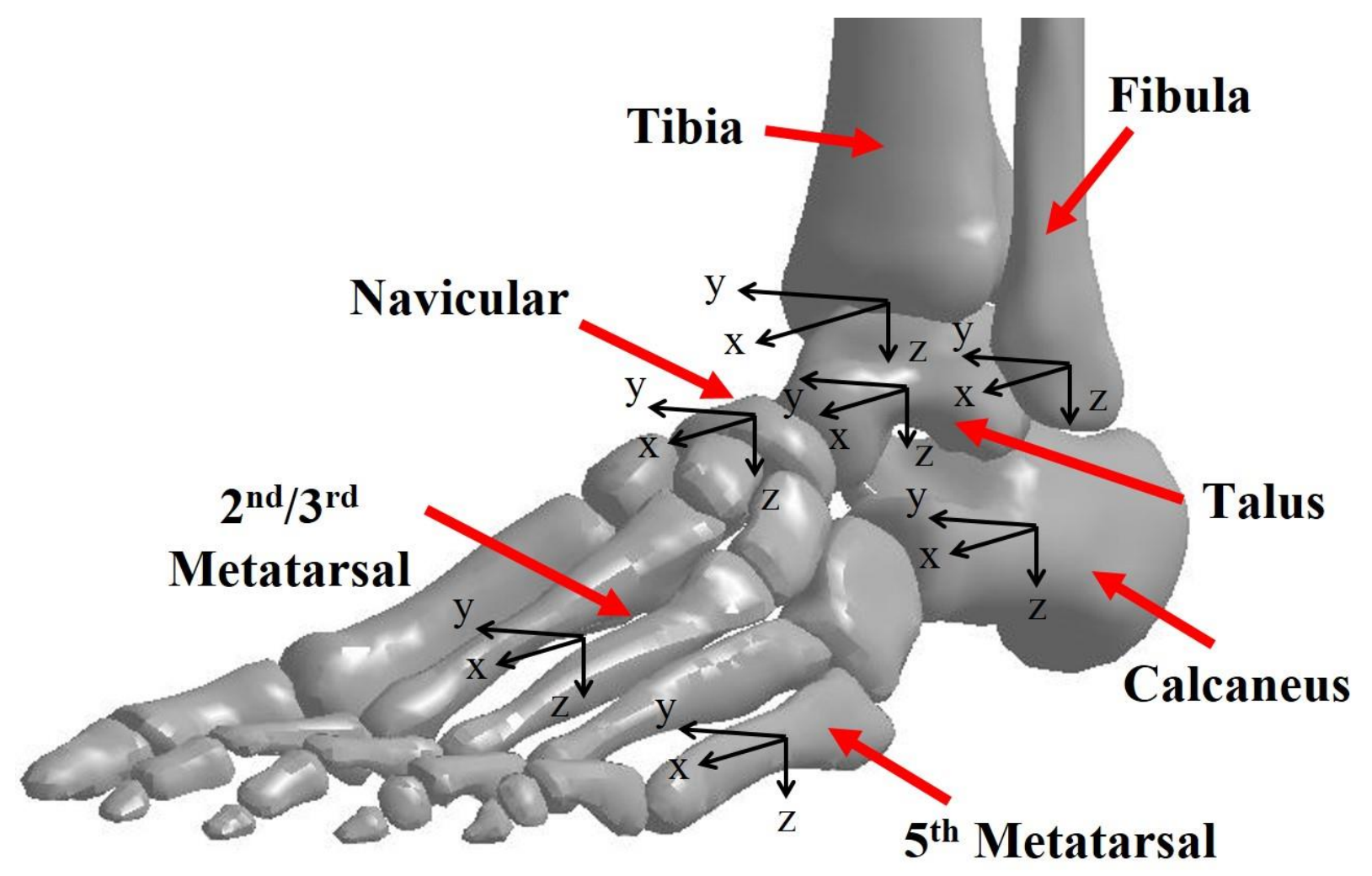

Figure 8: Local bone coordinate system definitions for foot and ankle bones in the initially neutral posture. Tibia coordinate system is defined using anatomic landmarks and bone geometry. From this tibia coordinate system, coordinate systems on other foot and ankle bones can be defined at their respective centroids (except fibula, defined at centroid of lateral malleolus), where all other bone coordinate axes are parallel to those of the tibia in the neutral ankle posture. Motion of these other bones are measured relative to the tibia coordinate system.

\subsubsection{Calculating Bone Kinematics and Clinical Ankle Angles}

According to Cappozzo et al. (1997), movement analysis should aim to reconstruct the instantaneous spatial position of bony landmarks in the laboratory (global) coordinate system. Orthogonal coordinate axes must be defined locally on bones and the instantaneous position and orientation (pose) of these local axes with respect to the global axes are determined experimentally during movement (Cappozzo et al., 1997). After experimental measurement of bone motion, information regarding the anatomical landmarks of the bones can then be added to determine the instantaneous position of these landmarks in the global coordinate system (Cappozzo et al., 1997). In this chapter, this movement analysis was adapted for the foot and ankle bones (tibia, fibula, 
calcaneus, talus, and navicular) using the transformation methodologies detailed by Shaw et al. (2009).

First, CT scans of the instrumented bones with 'marker clusters' attached (for this chapter, marker clusters refer to the aluminum marker cubes screwed into each bone) are performed. From these CT scans, the anatomical landmarks and geometry (inertial axes) of each bone can be digitized and local coordinate axes can be defined (Section 2.2.2), in the neutral ankle posture. Also, the positions of the marker cubes relative to each bone can be defined with transformation matrices that relate the coordinate systems defined on each marker cube to the local coordinate systems of each bone (Shaw et al., 2009). Then, while experiments are conducted, the position of the marker cubes are recorded with respect to the global coordinate system using some kind of optoelectronic stereometric system which reconstructs the position of points in a three-dimensional space (Cappozzo et al., 1997; Shaw et al., 2009). The transformation matrices relating each marker cube's coordinate system to the global coordinate system are calculated using initial position scans (before tests begin) and a least-squares pose estimator (Cappozzo et al., 1997; Shaw et al., 2009), where experimental errors (e.g., marker coordinate reconstruction errors and marker-bone relative motion errors due to soft tissue interactions) are additive. Lastly, with the transformation matrices relating marker cube position to the global coordinate system, the transformation matrices calculated in the CT scans relating the bone local coordinate systems to the marker cube coordinate systems are then used to determine the pose of each bone in the global coordinate system. Since this chapter aimed to determine the relative motion of each bone to the tibia, the motion of each bone in the global coordinate system was then transformed relative to $\mathrm{CS}_{\text {Tibia. }}$. Relative motion of the ankle bones was chosen to be described in the $\mathrm{CS}_{\text {Tibia }}$ since applied rotation and preload (detailed below) were input to a fixed proximal tibia and all other bones react to these inputs. This chain of homogeneous transformation matrices, to relate the relative motion of each instrumented bone to the tibia, is illustrated in Figure 9 among the representative coordinate axes depicted on the tibia (and attached marker), on an arbitrary bone (and attached marker), and in the global space. 


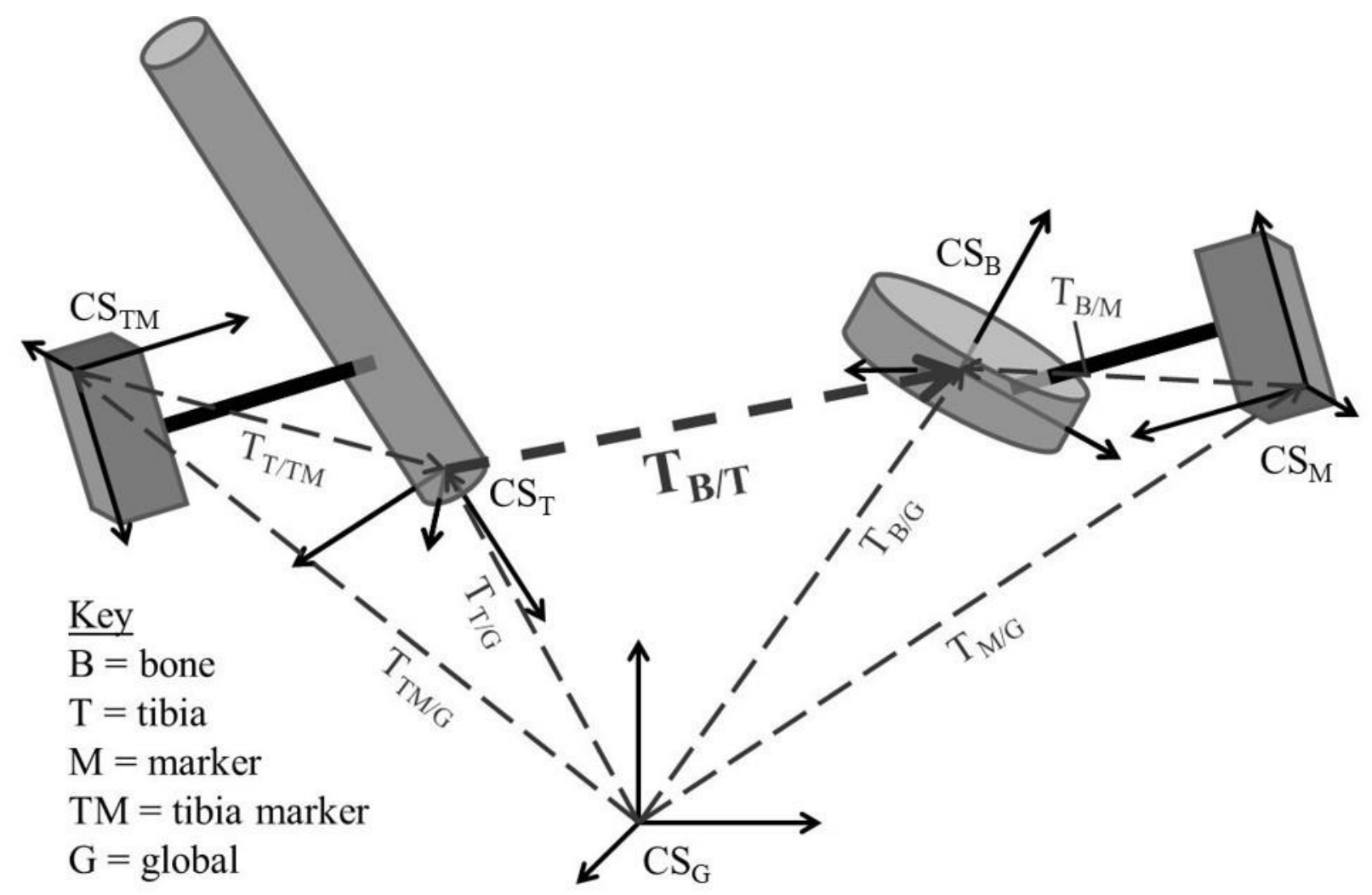

Figure 9: Process for transforming the coordinate system of each bone to the tibia coordinate system. Global refers to the coordinate system of the laboratory. The solid arrows indicate reference frames and the dashed arrows indicate transformation matrices relating reference frames. Each mount-bone pair was assumed to be a single rigid body.

Now to extract the kinematics of each ankle and foot bone during foot rotation testing, the methodologies detailed by Shaw et al. (2009) are applied to the geometrically defined local bone coordinate systems (Section 2.2.2) in the ankle. Let $\mathbf{i}_{\text {Tibia }}, \mathbf{j}$ Tibia, $\mathbf{k}_{\text {Tibia }}$ and $\mathbf{i}_{\text {Bone, }}, \mathbf{j}_{\text {Bone }}, \mathbf{k}_{\text {Bone }}$ be the unit vectors which describe the orientations of the $\mathrm{x}, \mathrm{y}$, and $\mathrm{z}$ axes of the tibia coordinate system ( $\left.\mathrm{CS}_{\text {Tibia }}\right)$ and any other bone's coordinate system $\left(\mathrm{CS}_{\mathrm{Bone}}\right)$ with respect to the global coordinate system, and $\mathbf{P}_{\text {Bone/Tibia }}=\left\langle\mathrm{x}_{\text {Bone/Tibia, }} \mathrm{y}_{\text {Bone/Tibia, }} \mathrm{Z}_{\mathrm{Bone} / \mathrm{Tibia}}\right\rangle$ be the vector which describes the position of the origin of $\mathrm{CS}_{\mathrm{B} \text { one }}$ with respect to $\mathrm{CS}_{\text {Tibia. }}$. Then the homogeneous transformation matrix from $\mathrm{CS}_{\text {Tibia }}$ to $\mathrm{CS}_{\text {Bone, }}$ which describes the geometric relationship between the position and orientation of $\mathrm{CS}_{\text {Bone }}$ with respect to $\mathrm{CS}_{\text {Tibia, }}$, is given by Equation (1). A chain of transformations (Figure 9) was used with additional coordinate systems defined on the bone marker cubes in the global coordinate system and the CT scans to calculate $\mathbf{T}_{\text {Tibia,Bone }}$ (Shaw et al., 2009). The columns of the first $3 \times 3$ submatrix of $\mathbf{T}_{\text {Tibia,Bone, which describes the difference in orientation between } \mathrm{CS}_{\text {Tibia }} \text { and }}$ 
$\mathrm{CS}_{\mathrm{Bone}}$, express the unit vectors of $\mathrm{CS}_{\mathrm{Bone}}$ in $\mathrm{CS}_{\text {Tibia }}$ Equation (2). The rows of the first $3 \times 3$ submatrix of $\mathbf{T}_{\text {Tibia,Bone }}$ express the unit vectors of $\mathrm{CS}_{\text {Tibia }}$ in $\mathrm{CS}_{\text {Bone }}$ Equation (3).

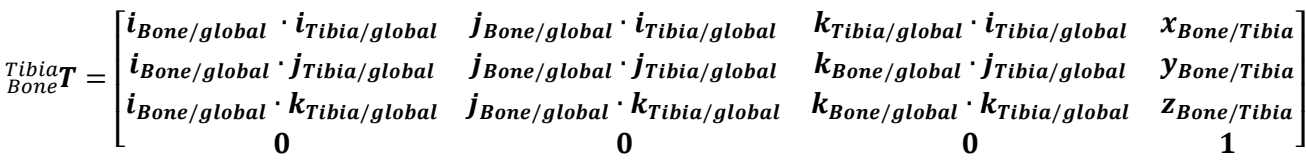

$$
\begin{aligned}
& \boldsymbol{T}_{\text {Tibia }, \text { Bone }}=\left[\begin{array}{cccc}
\left\{\boldsymbol{i}_{\text {Bone } / \text { Tibia }}\right\} & \left\{\boldsymbol{j}_{\text {Bone } / \text { Tibia }}\right\} & \left\{\boldsymbol{k}_{\text {Bone } / \text { Tibia }}\right\} & \left\{P_{\text {Bone } / \text { Tibia }}\right\} \\
0 & 0 & 0 & 1
\end{array}\right] \\
& \boldsymbol{T}_{\text {Tibia,Bone }}=\left[\begin{array}{cc}
\left\{\boldsymbol{i}_{\text {Tibia/Bone }}\right\}^{\prime} & x_{\text {Bone } / \text { Tibia }} \\
\left\{\boldsymbol{j}_{\text {Tibia/Bone }}\right\}^{\prime} & y_{\text {Bone } / \text { Tibia }} \\
\left\{\boldsymbol{k}_{\text {Tibia/Bone }}\right\}^{\prime} & z_{\text {Bone } / \text { Tibia }} \\
\{\boldsymbol{0}\}^{\prime} & 1
\end{array}\right]
\end{aligned}
$$

The linear (Equations (4), (5), and (6)) and angular (Equations (7), (8), and (9)) displacements of the bones from the neutral ankle posture to a non-neutral ankle posture were expressed as three linear displacements along the axes of $\operatorname{CS}_{\text {Tibia }}(\Delta x, \Delta y$, and $\Delta z)$ and three Euler Angles in the z-y$\mathrm{x}$ convention ( $\Delta$ yaw, $\Delta$ pitch, $\Delta$ roll).

$$
\begin{aligned}
& \Delta \mathrm{x}={ }_{\text {Bone }}^{\text {Tibia }} \boldsymbol{T}_{41} \\
& \Delta \mathrm{y}={ }_{\text {Bone }}^{\text {Tibia }} \boldsymbol{T}_{42} \\
& \Delta \mathrm{z}={ }_{\text {Bone }}^{\text {Tibia }} \boldsymbol{T}_{43} \\
& \text { syaw }=\tan ^{-1}\left(\begin{array}{l}
\text { Tibia } \\
\begin{array}{l}
\text { Bone } \\
\text { Toibia }_{21} \\
\text { Bone }
\end{array} \\
\boldsymbol{T}_{11}
\end{array}\right) \\
& \Delta \text { pitch }=\tan ^{-1}\left(\frac{-{ }_{\text {Bone }}^{\text {Tibia }} \boldsymbol{T}_{31}}{\sqrt{\text { Tibia }_{\text {Bone }} \boldsymbol{T}_{32}^{2}+{ }_{\text {Bone }}^{\text {Tibia }} \boldsymbol{T}_{33}^{2}}}\right) \\
& \text { soll }=\tan ^{-1}\left(\begin{array}{l}
\begin{array}{l}
\text { Tibia } \\
\text { Bone }
\end{array} \\
\begin{array}{l}
\text { Tibia } \\
\text { Bone }
\end{array} \\
\boldsymbol{T}_{33}
\end{array}\right)
\end{aligned}
$$

To relate the measured motion of the ankle bones in the geometric coordinate system definition to clinical definitions (Table 3), the ankle's rotation, flexion, and version angles were calculated from 
the relative motion of the calcaneus and tibia (Figure 10). Given the homogeneous transformation matrix, $\mathbf{T}_{\mathrm{Tib}, \mathrm{Cal}}$, which defines the position and orientation of the calcaneus' coordinate system (CS $\mathrm{CSal}_{\text {) }}$ with respect to $\mathrm{CS}_{\text {Tibia }}$ (Figure 11), components of $\mathbf{T}_{\text {Tib,Cal }}$ are extracted to calculate the clinical joint angles as detailed by Table 3 .

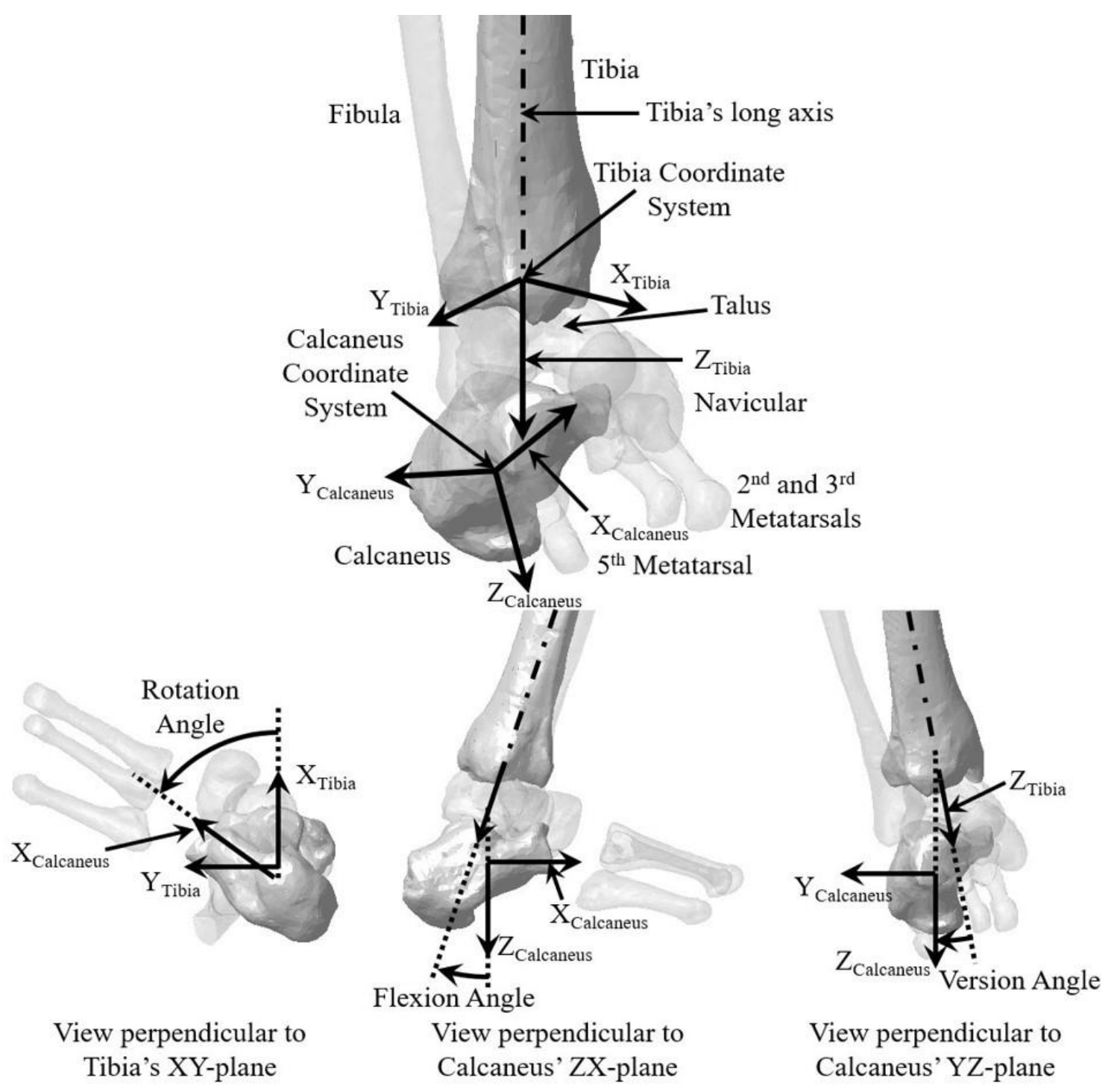

Figure 10: Top: Antero-Lateral view of the right leg in combined external rotation, dorsiflexion and eversion. Bottom: Definition of rotation, flexion, and version angles based on the coordinate systems of the tibia and calcaneus. Positive rotation, flexion, and version were external rotation, dorsiflexion, and eversion and negative rotation, flexion, and version were internal rotation, plantarflexion, and inversion. 


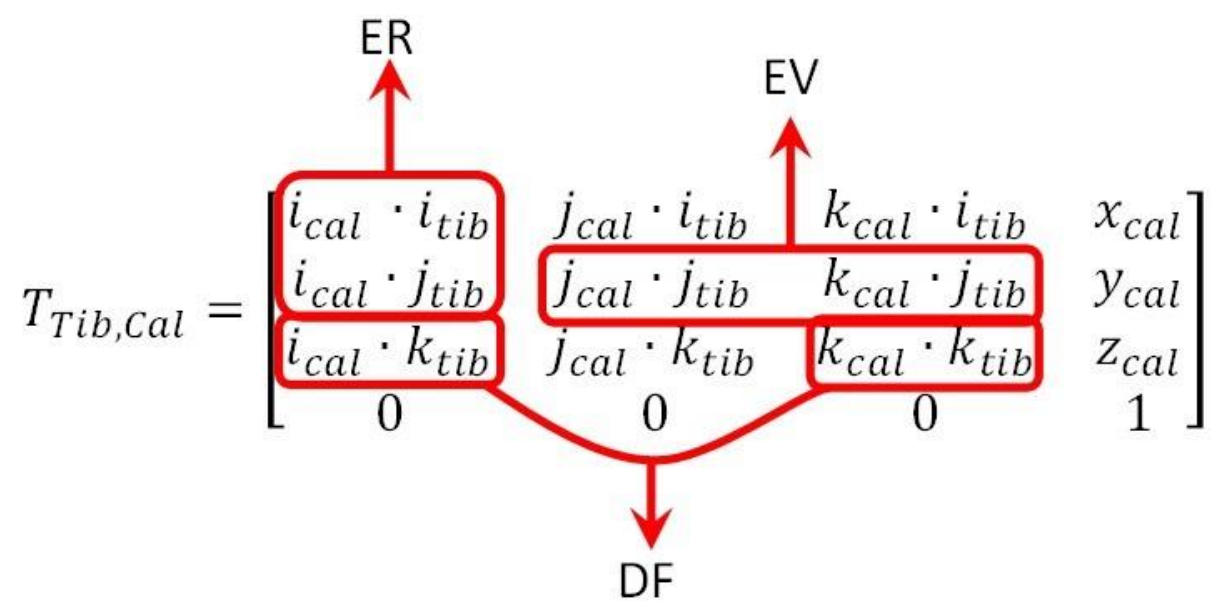

Figure 11: Components extracted from $\mathbf{T}_{\text {Tib,Cal }}$ to calculate joint angles $(\mathrm{ER}=$ external foot rotation, $\mathrm{EV}=$ ankle eversion, $\mathrm{DF}=$ ankle dorsiflexion).

To summarize, the ankle's rotation, flexion, and version angles were calculated from the motion of the calcaneus relative to the tibia in the geometrically defined coordinate systems (Table 3). Similar to the geometric approach for joint angle calculations in the knee proposed by Allard et al. (1997), Equations (10), (11), and (12) calculate these clinical angles instead for ankle rotation, flexion, and version based on the motion of the tibia and calcaneus. External/internal rotation of the foot in the ankle is calculated as the angle in the tibia's $x-y$ plane between the tibia's $x$-axis and the projection of the calcaneus x-axis on the tibia's x-y plane. Dorsi/plantarflexion of the ankle is calculated as the angle in the calcaneus z-x plane between the calcaneus z-axis and the projection of the tibia z-axis on the calcaneus z-x plane. E/inversion of the ankle is calculated as the angle in the calcaneus $y-z$ plane between the calcaneus z-axis and the projection of the tibia $z$-axis in the calcaneus y-z plane.

These clinical ankle angle calculations are closely related to the $\Delta$ yaw, $\Delta$ pitch, and $\Delta$ roll angles calculated in Equations (7), (8), and (9). Recall that Euler angles are a sequence of rotations. Yaw is the first rotation about the tibia's original z-axis, which is equivalent here to a rotation of the calcaneus about the tibia's long-axis (z-axis), which was defined as external/internal rotation of the foot/ankle. Subsequently, roll is the third rotation about the tibia's final $\mathrm{x}$-axis, which is equivalent here to a rotation of the tibia about the calcaneus' long-axis (x-axis), which was defined as e/inversion of the ankle. Pitch is the second rotation, about an intermediate axis, and the 
direction of this intermediate axis depends on the yaw angle. However, ankle flexion was defined as the rotation of the tibia about the calcaneus' medial-lateral axis (y-axis). Therefore, pitch and ankle flexion are not synonymous, as roll and ankle version or yaw and foot/ankle rotation are, but still closely related.

$$
\begin{aligned}
& \text { rotation }=-\tan ^{-1}\left(\frac{{ }_{\text {Calc }}^{\text {Cib }} \boldsymbol{T}_{21}}{{ }_{\text {Calc }} \boldsymbol{T}_{11}}\right) \\
& \text { flexion }=-\tan ^{-1}\left(\frac{\stackrel{\text { Tib }}{\text { Calc }} \boldsymbol{T}_{31}}{\operatorname{Calc}_{\text {Calc }} \boldsymbol{T}_{33}}\right) \\
& \text { version }=-\tan ^{-1}\left(\frac{\stackrel{\text { Tib }}{\text { Calc }} \boldsymbol{T}_{32}}{\text { Cib }_{\text {Calc }} \boldsymbol{T}_{33}}\right)
\end{aligned}
$$

Shaw et al. (2009) completed an assessment of kinematic displacement (linear and angular) data integrity to assess the accuracy of the displacement data and estimate the error. This error mainly depends on two factors: a) quality of optoelectronic data measured and b) integrity of bone marker cube hardware (Shaw et al., 2009). A similar assessment was done for the current study to determine possible accuracy deficiencies in the optoelectronic data collection, bone marker hardware instrumentation, and geometrically defined bone coordinate systems using repeated trials from the three specimens tested. This assessment will act to improve future experiments, aimed at studying injurious applied foot rotation, by determining sources of error and compliance among the optoelectronic data collection, hardware fixation (both on bone and overall test fixture), and geometric bone coordinate system definitions.

\subsubsection{Experimental Test Procedures, Boundary Condition Improvements, and Bone Kinematic Measurements}

To test the applicability and feasibility of the geometric method for defining coordinate systems detailed above (Section 2.2.2), internal and external rotation tests were performed on three cadaveric legs in a non-injurious, repeated trial protocol. This non-injurious test series was designed to confirm the utility of the geometric coordinate definitions in a small sample of cadaveric experiments. Furthermore, this small-scale study was designed to test the feasibility of 
the improved boundary conditions (detailed below) imposed on the legs during applied foot rotation. Various initial ankle postures (i.e. neutral, plantar/dorsiflexion, and e/inversion) were tested on each specimen. The goal of this small-scale, non-injurious test series was to measure bone-specific motion of a select number of pertinent ankle bones (tibia, fibula, calcaneus, talus, and navicular bones) in multiple initial ankle postures. This will determine the feasibility and applicability of the geometric coordinate definitions at describing ankle bone kinematics during applied foot rotation under functionally relevant boundary conditions. Experimental testing methods for this chapter are similar to those in Mait et al. (2015, 2017b), but are detailed below.

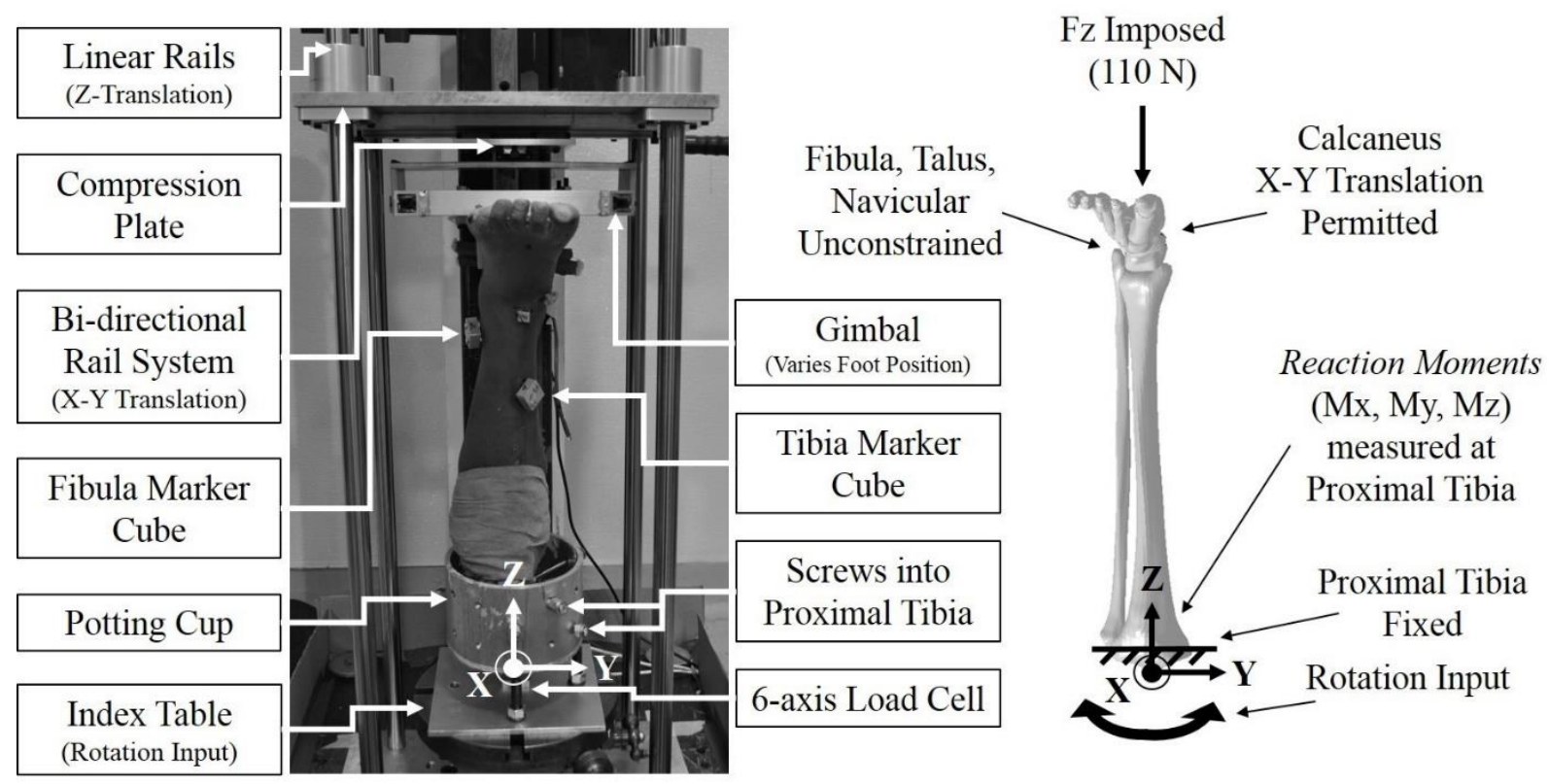

Figure 12: (Left) Experimental test rig, with a specimen in its neutral initial position, detailing each component or device used during testing as well as placement of these components in the rig. (Right) Schematic of the experimental boundary conditions with the locations of the input rotation and axial load. The global coordinate system $(\mathrm{X}-\mathrm{Y}-\mathrm{Z})$ is defined at the proximal tibia connection to the potting cup and load cell ( $\mathrm{Z}$-axis is coincident with the tibia long-axis; $\mathrm{X}$-axis inserts at the origin of the $\mathrm{Z}$ and $\mathrm{Y}$ axes and is aligned parallel to the second metatarsal). Fibula is unconstrained. Calcaneus transverse $(\mathrm{X}-\mathrm{Y})$ translation is permitted with the bi-directional rail system, and gross Z-translation is permitted with the linear rails. However, calcaneus rotation about the $\mathrm{X}$ and $\mathrm{Y}$ axes is constrained by the mount surrounding the bone.

A custom rig was designed to rotate the specimen to a desired effective angle of non-injurious foot rotation in the global X-Y-Z coordinate system. The global coordinate system was defined where the center of the tibial plateau was fixed to the center of the potting cup (Figure 12). A constant 
compressive load $(110 \mathrm{~N})$, chosen to not overly constrain the leg but still initiate ligament recruitment and joint congruency, was applied via static weight down the Z-axis.

The potting cup around the proximal tibia was attached to a rotary index table which was used to manually impart the desired quasi-static effective foot rotation about the Z-axis. Maximum effective foot rotation of $\pm 30^{\circ}$ was determined to be non-injurious (Wei et al., 2012b), yet greater than physiological range of motion (Nigg et al., 1990; Roaas and Andersson, 1982). A bidirectional linear rail system (Figure 12) attached to the calcaneus mount allowed the foot to translate in the global $\mathrm{X}$ and $\mathrm{Y}$ directions, and linear bearing tracks permitted motion in the Z-axis (vertical direction), thus allowing the $\mathrm{Z}$-axis of rotation to adjust to the natural axis of rotation in the leg (Funk, 2011; Rastegar et al., 1980).

The foot was mounted to this rail system using two gimbals with orthogonal rotation axes, originally parallel to the global coordinate system axes in a neutral ankle posture when specimens were placed into the test apparatus. Neutral posture was defined such that the first phalanx, approximate calcaneus centroid, and tibia long-axis formed a right angle in the sagittal plane. The gimbals (fixed to the calcaneus mount) allowed the foot to be locked in this neutral posture or rotated the calcaneus into varying degrees of eversion (EV), inversion (IV), dorsiflexion (DF), and plantarflexion (PF). These foot orientations were defined grossly as rotations of the calcaneus relative to the tibia within a nominal range of $10^{\circ}$ to $20^{\circ}$, used since within physiological range of motion of the ankle joint (Nigg et al., 1990; Roaas and Andersson, 1982).

A six-axis load cell (Model \#5024J, Robert A Denton, Inc., Rochester Hills, MI, USA) and rotary potentiometer (Model \#SP22GS, ETI Systems, Carlsbad, CA, USA), aligned between the centers of the potting cup and index table, measured forces and moments acting at the proximal tibia and the imposed effective foot rotation (Figure 12). To summarize the boundary conditions imposed, the proximal tibia was fixed, yet the fibula, talus, and navicular were unconstrained. The calcaneus was fixed in the calcaneus mount, thus restricting localized motion (translation and rotation) of the calcaneus. However, gross calcaneus translation was permitted through the rail system in all directions, and the gimbals permitted gross calcaneus rotation relative to the tibia to accommodate the initial ankle postures (neutral, EV, IV, DF, and PF). These functionally relevant boundary conditions address deficiencies in previous studies (Markolf et al., 1989; Michelson et al., 1997; 
Villwock et al., 2009; Wei et al., 2012b, 2010; Xenos et al., 1995), so that the leg is not artificially constrained and more realistic interactions among ankle bones and ligaments are attained.

In the initial neutral posture, all specimens were preconditioned before testing by rotating the index table for 10 cycles from $+30^{\circ}$ to $-30^{\circ}$, chosen to ensure binding within test fixture would not occur during testing yet still be non-injurious. The specimens were then either kept in neutral or placed into a desired configuration of EV, IV, DF, or PF. To measure bony kinematics, three adjacent sides on the surface of each motion-tracking marker cube (fixed to tibia, fibula, talus, navicular, and calcaneus) were digitized using a three-dimensional laser scanner (Model\#: RA-7330SI-2, Hexagon Metrology, Inc., North Kingstown, RI, USA) after placing the specimen in the desired initial ankle posture. Then, the specimens were rotated manually to the predetermined degree of quasi-static effective foot rotation by the index table. This final rotated position was held while the orientation and position (pose) of each bones' marker cubes were scanned again. The digitized scans of the three sides of each marker cube were considered the 'marker cluster' (Cappozzo et al., 1997; Shaw et al., 2009), defined in the global coordinate system. From these scans, the reconstructed marker cube coordinates were then related to the pose of each bone using the CT scans, pose estimator, and rigid-body transformation matrices (Sections 2.2.2 and 2.2.3). Then, the pose of all bones (except tibia) was transformed to $\mathrm{CS}_{\text {Tibia }}$ to calculate the relative motion of each bone to the tibia after applied rotation. External foot rotation (ER) was defined as the change in calcaneus yaw relative to the tibia long-axis (positive magnitude) in the transverse plane (Section 2.2.3), imposed by rotating the tibia internally with the rotary index table $\left(-30^{\circ}\right.$ effective foot rotation). Consequently, internal foot rotation (IR) was the change in calcaneus yaw relative to the tibia long-axis (negative magnitude), imposed by rotating the tibia externally $\left(+30^{\circ}\right.$ effective foot rotation). Since these were non-injurious tests, repeated tests were performed on multiple specimens to test the validity of the boundary conditions and kinematic measurement techniques.

With coordinate systems defined (Figure 8) for a left leg, such that the x-axis was positive anteriorly, y-axis was positive medially, and the z-axis was positive inferiorly, the measured displacements during testing (relative to the tibia) of each bone can be interpreted as follows. For linear displacements (translations), $+\Delta \mathrm{x}$ corresponded to anterior motion and $-\Delta \mathrm{x}$ to posterior motion, $+\Delta \mathrm{y}$ corresponded to medial motion and $-\Delta \mathrm{y}$ to lateral motion, and $+\Delta \mathrm{z}$ corresponded to 
inferior motion and $-\Delta \mathrm{z}$ to superior motion. For angular displacements (rotations), $\pm \Delta y a w$ corresponded to rotation in the transverse plane (for the calcaneus, $+\Delta$ yaw was ER and $-\Delta y a w$ was IR), $\pm \Delta$ pitch corresponded to rotation in the sagittal plane, and $\pm \Delta$ roll corresponded to rotation in the coronal plane.

Post-test necropsies were performed to confirm the lack of ligament damage and bone fracture. The ATiFL, PTiFL, IOM, ATaFL, PTaFL, CFL, superficial deltoid complex, and deep deltoid complex were all inspected.

\subsubsection{Experimental and Kinematic Measurement Accuracy Assessment}

Repeated tests were performed on each specimen for the initially neutral posture with imposed maximum ER ( $-30^{\circ}$ effective foot rotation). These repeated trials were used to assess the utility of the boundary conditions, test fixture, instrumentation hardware, and the geometric coordinate system definition used in this proof-of-concept study. Absolute and percent differences of the angular and linear displacements for the fibula, calcaneus, talus, and navicular bones between these two repeated trials on each specimen were determined. This was used to nominally determine the inaccuracy of the geometric coordinate system definition and compliance within the test fixture hardware and kinematics instrumentation on each bone.

To estimate the error in the geometric coordinate system determination, anatomical landmark points (centroids of fibula, calcaneus, talus, and navicular; tibia origin; and anterior-inferior tip of the medial malleolus) determined by the experimenter and automatically calculated by Mimics 18.0 were digitized for five separate trials (i.e. five centroids for each bone and five tibia origins) in the same specimen's CT scan. The mean position $\left(x_{\text {mean }}, y_{\text {mean }}, z_{\text {mean }}\right)$ of each point was calculated with the points digitized from the five trials $\left(i=5 ; x_{i}, y i, z_{i}\right)$. Maximum resultant (distance) deviation from this mean position was then determined with $d_{i}=$ $\sqrt{\left(x_{\text {mean }}-x_{i}\right)^{2}+\left(y_{\text {mean }}-y_{i}\right)^{2}+\left(z_{\text {mean }}-z_{i}\right)^{2}}$. From these points (from the five trials), the tibia local x, $\mathrm{y}$-, and z-axes $\left(i=5, k=3 ; \mathbf{u}_{i, \mathrm{k}}\right)$ were determined as described in Section 2.2.2 and the mean $\mathrm{x}-$, $\mathrm{y}$-, and $\mathrm{z}$ - axes $\left(\mathrm{k}=3 ; \mathbf{u}_{\text {mean, } \mathrm{k}}\right)$ were then determined. Maximum angular deviation from the mean 
axes was determined with $\theta_{i}=\cos ^{-1}\left(\mathbf{u}_{\text {mean }} \cdot \mathbf{u}_{i}\right)$. An illustration of this process is given below in Figure 13.

\section{Resultant (distance) Deviations}

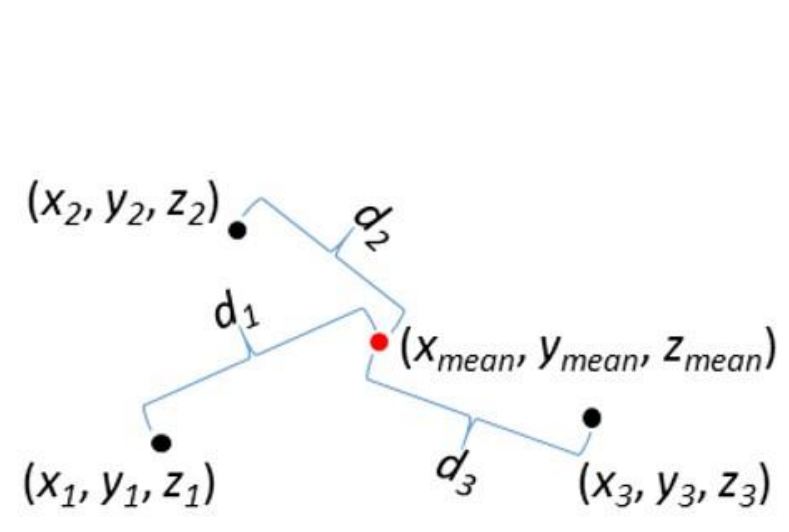

\section{Angular Deviations}

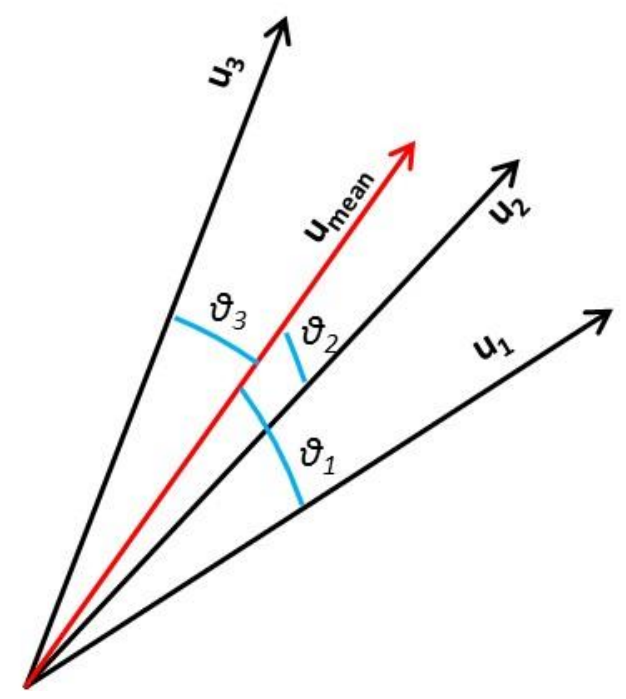

Figure 13: Illustration of maximum deviation from the mean for points (distance, in $\mathrm{mm}$ ) and for the initial tibia local axes (angular, in ${ }^{\circ}$ ) determined by Mimics 18.0 and the experimenter.

\subsection{Results}

Post-test necropsies revealed no ligament tears, avulsions, or bone fractures on any specimen. The absolute and percent differences between the linear $(\Delta x, \Delta y$, and $\Delta z)$ and angular ( $\Delta y a w, \Delta$ pitch, $\Delta$ roll) displacements of the fibula, calcaneus, talus, and navicular for each repeated test on all specimens are shown in Table 4. Though the majority of the absolute differences are submillimeter and sub-degree in magnitude, there are several displacement measurements between these repeated trials where magnitudes of differences are greater than $1 \mathrm{~mm}$ or $1^{\circ}(2.4 \mathrm{~mm}$ and $4.2^{\circ}$ maximum), most noticeably for displacements of the calcaneus and navicular. 
Table 4: Absolute and percent differences for repeated ER tests on each specimen. Differences among the linear and angular displacements from the fibula, calcaneus, talus, and navicular are reported.

\begin{tabular}{|c|c|c|c|c|c|c|c|c|c|c|c|c|}
\hline Specimen \#, Trial & $\begin{array}{c}\text { ib_ayaw } \\
{\left[{ }^{\circ}\right]}\end{array}$ & 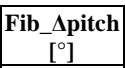 & \begin{tabular}{|c|} 
Fib_Aroll \\
$\left.{ }^{\circ}{ }^{\circ}\right]$
\end{tabular} & $\begin{array}{c}\begin{array}{c}\text { Fib_bx } \\
{[\mathrm{mm}]}\end{array} \\
\end{array}$ & $\begin{array}{c}\text { Fib_Ay } \\
{[\mathbf{m m}]}\end{array}$ & $\begin{array}{c}\text { Fib_Az } \\
{[\mathbf{m m}]}\end{array}$ & $\begin{array}{c}\text { Cal_tyaw } \\
{\left[{ }^{\circ}\right]}\end{array}$ & \begin{tabular}{|c} 
Cal_spitch \\
{$\left[{ }^{\circ}\right]$}
\end{tabular} & \begin{tabular}{|c|} 
Cal_troll \\
$\left.{ }^{\circ}{ }^{\circ}\right]$
\end{tabular} & $\begin{array}{c}\text { Cal_Ax } \\
{[\mathrm{mm}]}\end{array}$ & \begin{tabular}{|c} 
Cal_Ay \\
{$[\mathbf{m m}]$} \\
\end{tabular} & $\begin{array}{c}\text { Cal_Az } \\
{[\mathrm{mm}]} \\
\end{array}$ \\
\hline 616L, ER30 Trial 1 & -5.524 & -2.490 & -1.322 & -5.707 & -0.092 & -0.086 & -26.355 & 0.944 & 0.236 & -2.557 & 8.065 & 0.493 \\
\hline 616L, ER30 Trial 2 & -5.106 & -2.466 & -1.133 & -5.632 & -0.295 & 0.234 & -26.423 & 1.297 & 0.492 & -2.826 & 7.516 & 0.401 \\
\hline Absolute Difference & -0.418 & -0.024 & -0.190 & -0.074 & 0.204 & 0.148 & 0.068 & -0.354 & -0.255 & 0.269 & 0.549 & 0.093 \\
\hline Perce & 7.864 & 0.957 & 15.469 & 1.314 & 105.324 & 92.599 & 0.257 & 31.553 & 70.076 & 9.986 & 7.048 & 20.758 \\
\hline 7391 & -4.410 & .105 & -1.559 & 35 & 617 & 7 & -22.312 & & 29 & .052 & & 4.8 \\
\hline $739 \mathrm{~L}$ & -3.769 & -1.250 & -1.703 & -2.192 & 1.354 & 99 & -22.897 & 6.491 & 2.442 & 4.653 & 0.431 & 3.931 \\
\hline $\mathrm{Abs}$ & .642 & 146 & 0.144 & .757 & -0.736 & 47 & 85 & .299 & 1.088 & 2.399 & -0.337 & 0.903 \\
\hline & 689 & 389 & 8.829 & 1.724 & 74.695 & .826 & 2.588 & 2.240 & 36.436 & 40.996 & 28.221 & 20.598 \\
\hline & .781 & 16 & .035 & 22 & 1.385 & 578 & -21.737 & .926 & 0.121 & 5.101 & 6.395 & 3.61 \\
\hline & -7.708 & -1.169 & -1.179 & -2.785 & 1.571 & .360 & -22.797 & 1.568 & 0.222 & 5.056 & $\begin{array}{l}6.688 \\
\end{array}$ & 3.735 \\
\hline Absc & -0.072 & 0.153 & 0.145 & 0.063 & -0.187 & 0.219 & 1.061 & 0.357 & -0.101 & 0.045 & -0.293 & -0.117 \\
\hline Per & 0.935 & 13.989 & 13.066 & 2.298 & 12.631 & 46.595 & 4.763 & 20.462 & 58.829 & 0.880 & 4.478 & 3.179 \\
\hline Spe & $\begin{array}{c}\text { Cal_uyaw } \\
{\left[{ }^{\circ}\right]}\end{array}$ & $\begin{array}{c}\text { Tal_spitch } \\
{\left[{ }^{\circ}\right]}\end{array}$ & $\begin{array}{c}\text { Tal_Aroll } \\
\left.{ }^{\circ}{ }^{\circ}\right]\end{array}$ & $\begin{array}{c}\text { Tal__t } \\
{[\mathbf{m m}]}\end{array}$ & [mm] & {$[\mathrm{mm}]$} & $\begin{array}{c}\text { Nav_Ayaw } \\
{\left[{ }^{\circ}\right]}\end{array}$ & $\begin{array}{c}\text { Nav_Lpitch } \\
{\left[{ }^{\circ}\right]}\end{array}$ & $\underset{\left[{ }^{\circ}\right]}{\text { Nav_Aroll }}$ & $\begin{array}{c}\text { Nav_ux } \\
{[\mathrm{mm}]}\end{array}$ & $\begin{array}{c}\text { Nav_Ay } \\
{[\mathbf{m m}]}\end{array}$ & $\begin{array}{c}\text { Nav_uz } \\
{[\mathrm{mm}]}\end{array}$ \\
\hline 616 & -19.374 & 0.268 & 2.604 & 3.459 & 0.114 & -0.116 & -22.279 & 0.407 & 2.922 & 10.829 & -9.493 & 1.564 \\
\hline 61 & 3.234 & 432 & 2.981 & .428 & -0.364 & 0.210 & -24.198 & 4.619 & 1.903 & 9.756 & -8.871 & 2.189 \\
\hline & -1.140 & 164 & .378 & 032 & 0.478 & 26 & 1.919 & -4.212 & 1.019 & 1.073 & -0.622 & -0.625 \\
\hline Perce & 6.064 & 46.772 & 13.530 & 0.918 & 382.619 & 699.275 & 8.259 & 167.573 & 42.243 & 10.426 & 6.777 & 33.292 \\
\hline & .269 & .185 & 1.608 & 2.902 & -2.230 & -0.439 & -14.083 & 7.940 & 5.144 & 7.290 & 0.960 & -4.280 \\
\hline 739L, & -14.834 & 10.154 & 2.151 & 2.860 & -1.394 & 0.620 & -14.537 & 7.856 & 6.131 & 7.716 & \begin{tabular}{|l|}
-10.916 \\
\end{tabular} & -1.778 \\
\hline Absolt & 0.565 & 2.031 & -0.543 & 0.042 & -0.836 & -1.059 & 0.455 & 0.084 & -0.987 & -0.425 & -0.045 & -2.502 \\
\hline Perce & 3.884 & 18.183 & 28.873 & 1.458 & 46.156 & 1172.662 & 3.178 & 1.059 & 17.515 & 5.667 & 0.408 & 82.599 \\
\hline & 700 & 6.291 & -0.839 & 4.309 & -0.825 & 1.379 & -12.206 & 3.138 & 1.877 & 8.290 & $\mid-12.615$ & -2.072 \\
\hline & .033 & 4.446 & -1.025 & 4.966 & -0.227 & 1.333 & -15.800 & 2.190 & 1.052 & 8.551 & -9.584 & -0.218 \\
\hline & 1.334 & 1.845 & 0.186 & -0.657 & -0.599 & 0.045 & 3.594 & 0.948 & 0.825 & -0.261 & -3.031 & -1.854 \\
\hline Percent Difference & 8.149 & 34.364 & 9.947 & 14.172 & 113.814 & 3.356 & 25.666 & 35.577 & 56.367 & 3.105 & 27.312 & 161.93 \\
\hline
\end{tabular}

As expected, accumulation of error relied on the experimenter's ability to determine the anatomical landmark in question. The maximum deviations from the mean (Table 5) were greatest for picking the anterior-inferior point on the medial malleolus $(0.73 \mathrm{~mm})$ and the tibia origin $(0.25 \mathrm{~mm})$, where the tibia z-axis intersected with the external geometry of the tibial plafond. This error propagated further into the angular deviations for determining the $\mathrm{x}$ - and $\mathrm{y}$ - axes of the tibia since defining those axes depended on the experimenter's ability for picking the correct anatomical landmark point on the medial malleolus and tibial plafond. Not surprisingly, deviations from the mean were small for points (centroids of the fibula, talus, calcaneus, and navicular) and axes (tibia z-axis) calculated automatically by Mimics 18.0 (Table 5). 
Table 5: Error estimation with resultant deviations from the mean for geometric coordinate system definition. Centroids of fibula, calcaneus, talus, and navicular calculated automatically by

Mimics 18.0. Tibia z-axis calculated automatically by Mimics 18.0, but origin and medial malleolus points selected by experimenter (repeated selection of these points 5 times).

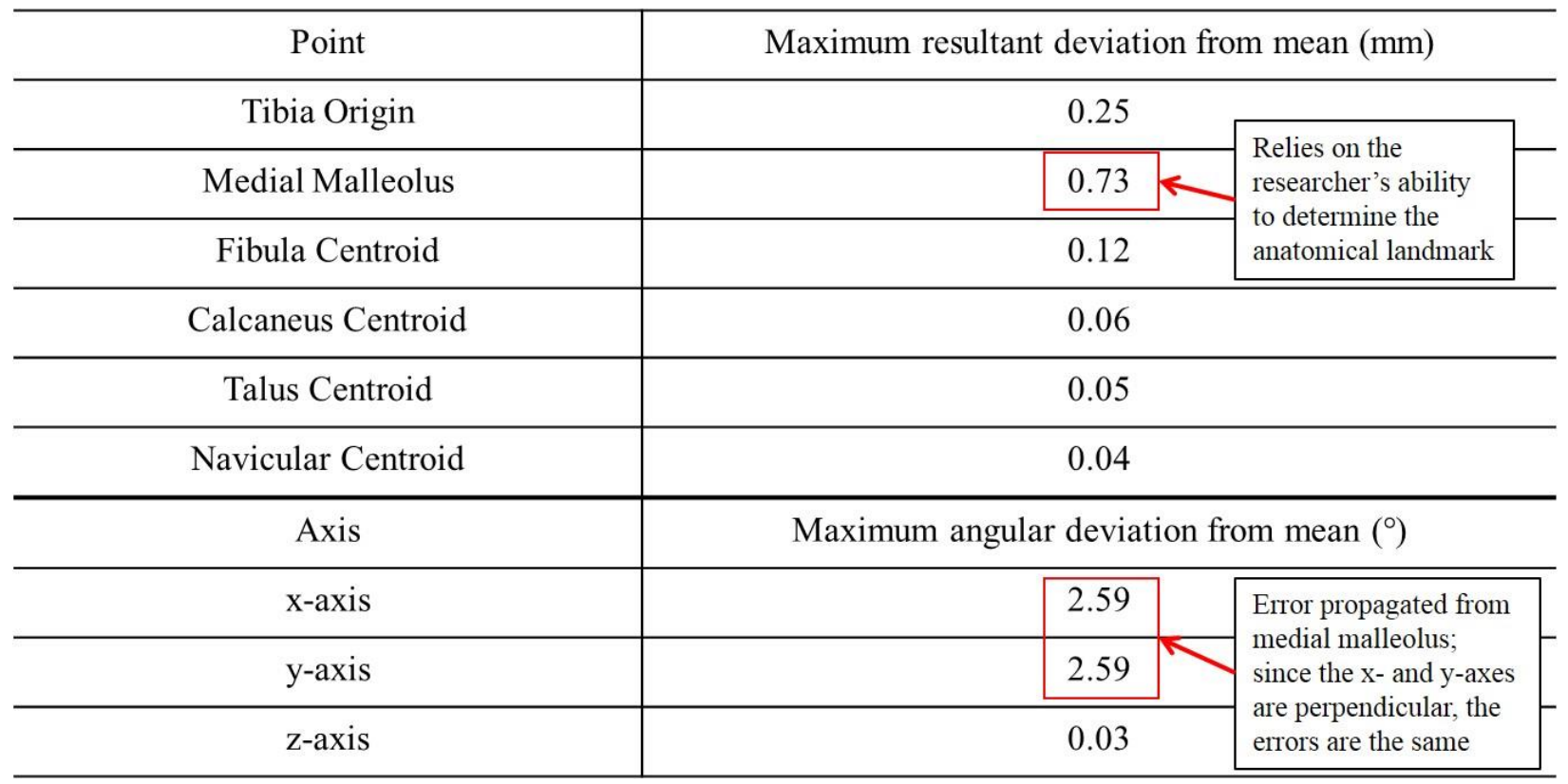

The changes in position and orientation (linear and angular displacements) of the instrumented bones (fibula, talus, navicular, and calcaneus) after ER and IR from initially neutral, everted, inverted, dorsiflexed, and plantarflexed ankle postures were calculated for all three specimens relative to the tibia coordinate system (Figure 14 and Figure 15). All calcaneus $\Delta$ yaw rotations (synonymous to ER and IR, see Section 2.2.3) were less in magnitude than the applied tibia rotation (effective foot rotation of $\pm 30^{\circ}$ ) with a minimum of $20.3^{\circ}\left(739 \mathrm{~L} / \mathrm{EV} / \mathrm{ER}\right.$ ) and maximum of $28.5^{\circ}$ (616L/DF/ER). 


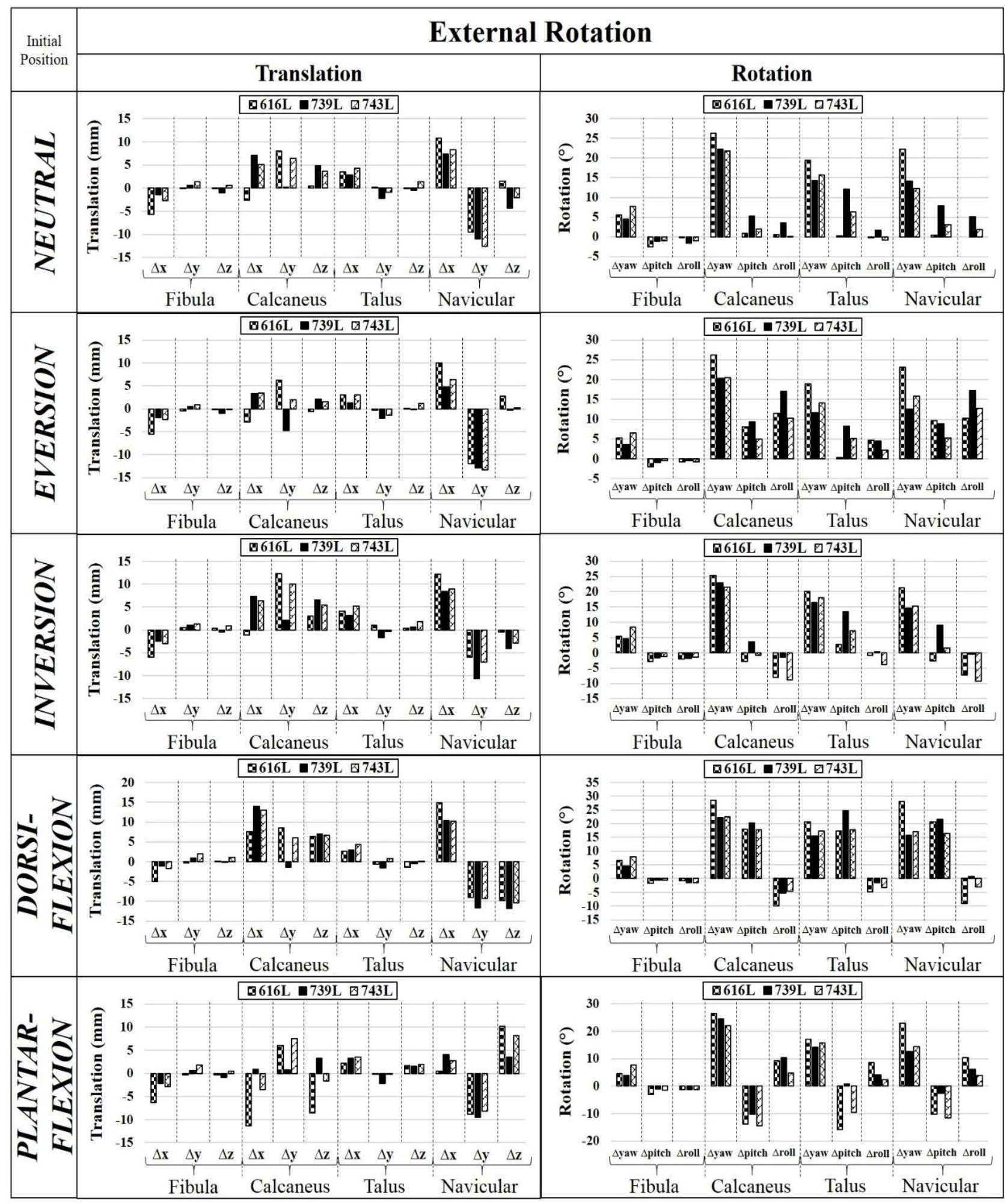

Figure 14: Calculated changes in linear $(\Delta \mathrm{x}, \Delta \mathrm{y}, \Delta \mathrm{z})$ and angular $(\Delta \mathrm{yaw}, \Delta \mathrm{pitch}, \Delta$ roll $)$ displacements of the fibula, calcaneus, talus, and navicular bones relative to the tibia coordinate system after ER. Neutral, everted, inverted, dorsiflexed, and plantarflexed initial ankle postures are reported. 


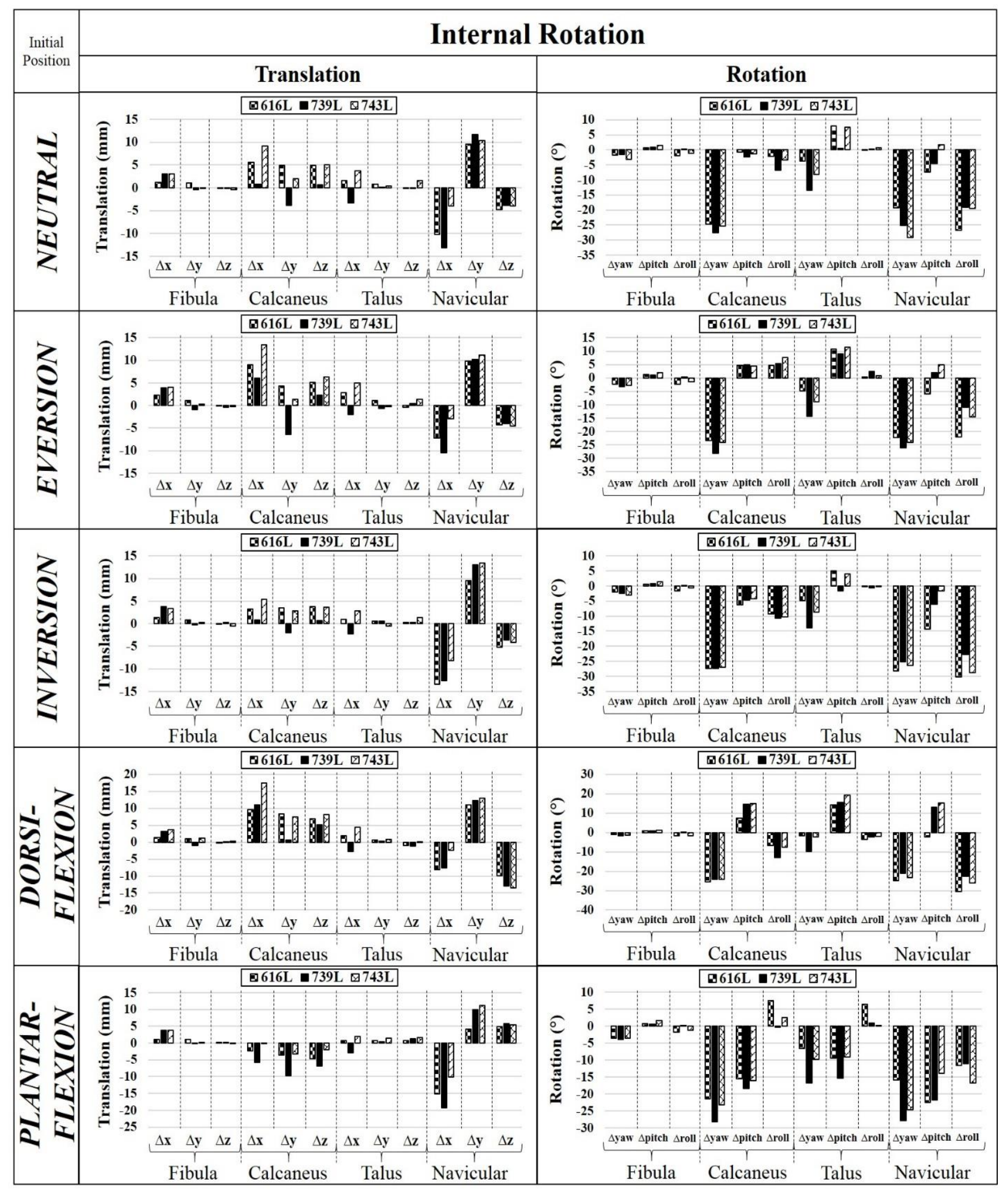

Figure 15: Calculated changes in linear $(\Delta \mathrm{x}, \Delta \mathrm{y}, \Delta \mathrm{z})$ and angular ( $\Delta \mathrm{yaw}, \Delta \mathrm{pitch}, \Delta \mathrm{roll})$ displacements of the fibula, calcaneus, talus, and navicular bones relative to the tibia coordinate system after IR. Neutral, everted, inverted, dorsiflexed, and plantarflexed initial ankle postures are reported. 
For all initial ankle postures (Figure 14 and Figure 15), the fibula translated anteriorly $(+\Delta x$; maximum $=4.0 \mathrm{~mm}, 743 \mathrm{~L} / \mathrm{EV} / \mathrm{IR})$ after $\mathrm{IR}$ but posteriorly $(-\Delta \mathrm{x} ;$ minimum $=-6.3 \mathrm{~mm}$, 616L/PF/ER) after ER. For all initial ankle postures, the talus translated anteriorly after ER. The navicular, across all specimens and foot positions, translated anteriorly and laterally $(-\Delta y)$ when subjected to ER, but posteriorly and medially $(+\Delta y)$ when subjected to IR. Navicular linear and angular displacements were larger in magnitude than all other bones relative to the tibia. Overall, the talus experienced larger $\Delta$ yaw magnitudes after ER than IR (ER maximum: 20.6 ${ }^{\circ}$, 616L/DF/ER; IR maximum: $\left.16.7^{\circ}, 739 \mathrm{~L} / \mathrm{PF} / \mathrm{IR}\right)$. The talus pitched away from the tibia after plantarflexion $\left(-15.7^{\circ} \Delta\right.$ pitch maximum), but toward after dorsiflexion $\left(24.8^{\circ} \Delta\right.$ pitch maximum).

Three-dimensional reconstructions (using Mimics 18.0) of the five instrumented bones were developed for a qualitative comparison of bone kinematics after preload and ER across a few initial ankle postures (Figure 16). Initial eversion rolled the talus toward the fibula, thus decreasing talofibular diastasis. However as the fibula displaced posteriorly, both talofibular and tibiofibular diastasis increased after applied ER. Initial dorsiflexion pitched the talus toward the tibia and displaced the fibula superiorly, and the fibula displaced again but posteriorly after applied ER, thus increasing tibiofibular diastasis. 


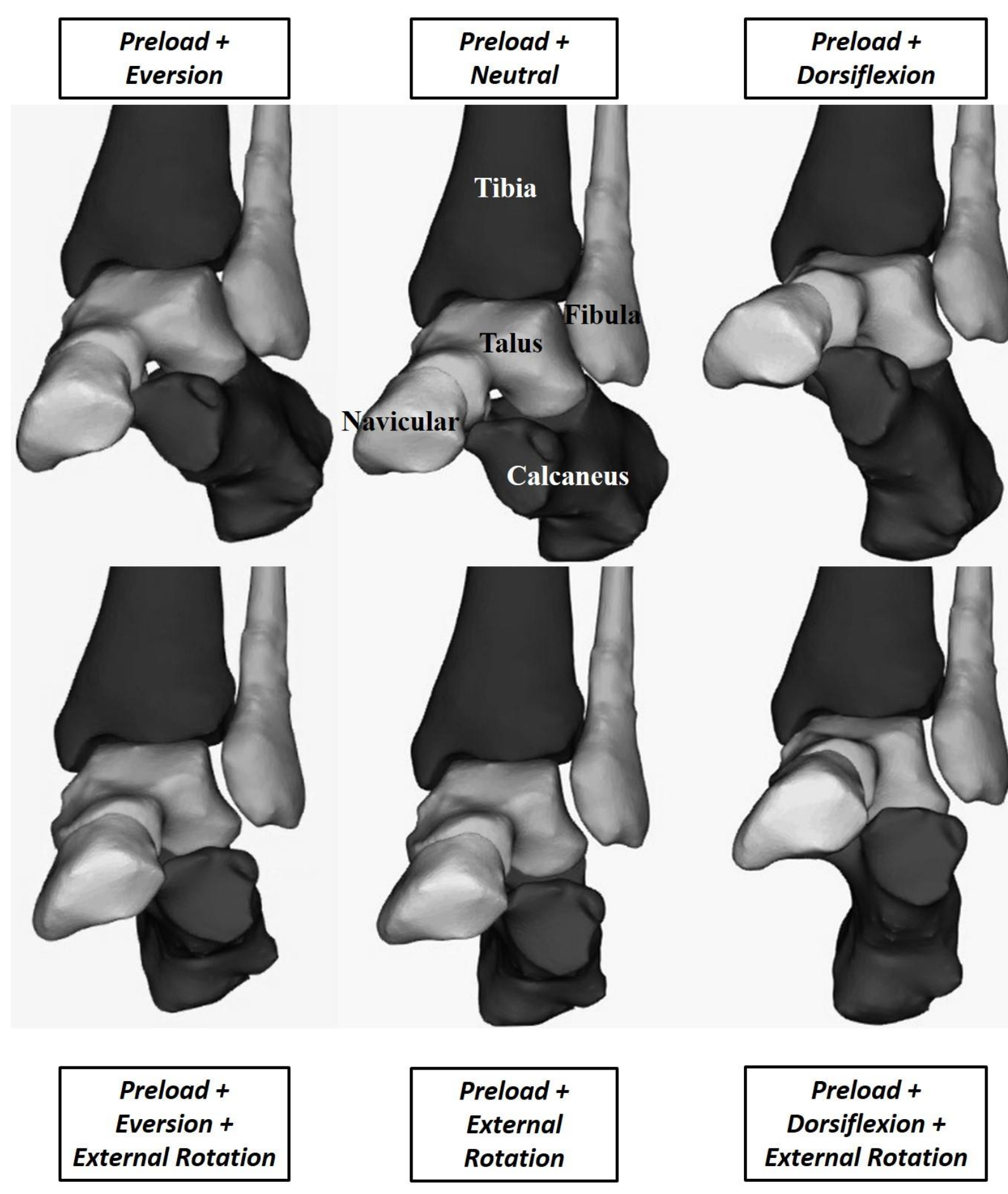

Figure 16: Comparison of the three-dimensional reconstruction of bones (specimen 616L) for tests starting from the initially neutral, everted, or dorsiflexed postures, then externally rotated. Reconstructions of bones begin with the leg axially compressed (110 N preload). 


\subsection{Discussion and Conclusions}

In this chapter, a coordinate system based on bone geometry and anatomical landmarks was defined locally on several foot and ankle bones. With this geometric coordinate system definition, clinically interpretable ankle rotations were quantified based on the relative kinematics of the calcaneus and the tibia. By capturing the relative motion of several foot and ankle bones in the geometrically defined coordinate system, ligament elongation can be inferred. Additionally, foot rotation was applied to cadaveric legs under functionally relevant boundary conditions which allowed the axis of rotation in the leg to move based on the natural bony motion of the foot-ankle complex (Funk, 2011; Rastegar et al., 1980).

Experiments employing artificially restrictive boundary conditions cannot accurately nor realistically re-create ankle mechanical response during applied foot rotation. Whether rigidly constraining the fibula to the tibia or preventing foot translation (Markolf et al., 1989; Michelson et al., 1997; Villwock et al., 2009; Wei et al., 2012b; Xenos et al., 1995), these artificially restrictive boundary conditions impair the ankle's ability to naturally respond to loading. Fixing the fibula to the tibia prevents natural fibula motion relative to the tibia, which creates unreliable tibiofibular interactions such as diastasis, the main indicator for syndesmotic injury. Preventing foot translation denies the foot the ability to react naturally to loading by constraining ligamentous and bony interactions, which could alter the mechanical response of the ankle. While using functionally relevant boundary conditions of an unconstrained fibula and permitted foot translation (Mait et al., 2015, 2017b), this chapter showed that, at a non-injurious level of applied foot rotation, pertinent kinematic data could be measured during applied foot rotation experiments (Objective 1).

Though joint coordinate systems are valuable for overall kinematic descriptions of human leg motion (Siegler et al., 1988; Wei et al., 2011; Wu et al., 2002), it is difficult to distinguish subtle bone-to-bone kinematic phenomena (e.g., tibiofibular diastasis) experienced during ankle rotation, especially translations of the fibula on the order of less than $7 \mathrm{~mm}$ (Figure 14 and Figure 15). These studies provide a gross ankle coordinate system defined for selected bones relative to each other, but not for all pertinent individual bones relative to one consistently defined bone coordinate system. The tibia coordinate system was chosen as this consistently defined bone coordinate 
system since all other ankle bones respond to the input tibia rotation causing foot rotation. Therefore, the definition of an individual, geometry-based coordinate system for all ankle bones was necessary (Objective 1) to measure subtle linear and angular displacements (Figure 14, Figure 15, and Figure 16). This geometrically defined local coordinate system convention on each pertinent foot and ankle bones allows for quantification of relative bone motion, from which ligament elongations and recruitment can be inferred. Also, the geometric coordinate system convention allows for interpretation of relative bone rotations in clinically defined terms (i.e., ankle rotation, flexion, and version), which will prompt descriptions of measured bone kinematics in terms conducive to ankle injury diagnosis, propagation, and tolerance in multiple ankle postures.

Kinematic motions among ankle bones during non-injurious foot rotation in varying initial ankle postures were quantified. The direction of fibula translation was observed to change with the direction of foot rotation, and the range of rotation of the talus was observed to be less during IR than during ER (Figure 14 and Figure 15). Pre-rotation ankle posture may influence the ankle bones' motions during rotation. The talus was observed (Figure 14 and Figure 16) to pitch into the ankle mortise during ER of a dorsiflexed foot $\left(24.8^{\circ} \Delta\right.$ pitch maximum), consequently increasing tibiofibular diastasis, which may increase the risk of syndesmotic ligament injury. However, the talus pitched away from the tibia after plantarflexion $\left(-15.7^{\circ} \Delta\right.$ pitch maximum), indicating the syndesmosis joint could be protected in plantarflexion. This outcome could indicate an increased risk of syndesmotic ligament injury in dorsiflexion by forcing the talus into the syndesmosis joint (Figure 14 and Figure 16). Conversely, an initially plantarflexed posture could indicate a protective state for the distal tibiofibular syndesmosis (Figure 14). Yet, external rotation of the foot remains the main contributor to tibiofibular and talofibular diastasis for initially neutral, everted, and dorsiflexed legs (Figure 16). This research is the first cadaveric study, to the author's knowledge, to directly measure six-degree-of-freedom bone-specific motion of all ankle bones in various ankle postures with an unconstrained fibula and permitted foot rotation.

For future clinical use, the kinematics displayed in Figure 14 and Figure 15 indicate bounds of bone motion relative to the tibia in a set of three intact, non-injured cadaveric specimens. The fibula translated less than $7 \mathrm{~mm}$ in all directions and rotated less than $10^{\circ}$ about all axes during both ER and IR, possibly indicating ATiFL, PTiFL, and IOM compliance prior to injury. The 
navicular linear and angular displacement magnitudes were often greatest, across all initial ankle postures, among all bones, which could indicate a possible increased compliance in the superficial deltoid complex relative to other ankle ligaments during applied foot rotation. This kinematic data has utility in future ankle injury diagnoses comparing uninjured limbs to injured limbs. Despite the noted inaccuracies in the test fixture and coordinate system definition (Table 4 and Table 5), the order of magnitude of linear and angular displacements ( 0 to $15 \mathrm{~mm}$ and 0 to $30^{\circ}$ ) of ankle bones during applied foot rotation was determined in this chapter for non-injurious tests. Clinicians can use this information to know the difference between bone translations and rotations (relative to the leg, or tibia) from uninjured and injured limbs, especially for the fibula relative to the tibia (less than $7 \mathrm{~mm}$ and $10^{\circ}$ ) when diagnosing syndesmotic injury.

The selection of local coordinate systems for the quantification of ankle bone kinematics is challenging. The amorphous, variable shape of the ankle bones confounds attempts to define local bone-based coordinate systems that are anatomically relevant, technically feasible, and consistently applicable. However, geometric properties and anatomical landmarks, used in this chapter, may provide an objective, consistent method to define relative kinematics of bones (Camacho et al., 2002). Though consistent, the method for defining geometrically based coordinate system conventions does contain inaccuracies, where choice of anatomical landmarks depends on the experimenter (Table 5). These inaccuracies propagate through to the definition of local bone coordinate axes, however the applicability of this geometric coordinate system convention outweighs the inaccuracies. With the geometric convention, ankle motions are clinically interpretable and relative bone-specific motion is quantified which makes inferring ligament elongation possible. These inaccuracies for defining anatomical landmarks can be limited, in part, with one experimenter picking all geometry and landmarks for coordinate system definitions.

Even with quantifying the inaccuracies associated with defining the geometric coordinate system convention (resultant and angular deviations from the mean; Table 5), not all inaccuracy was accounted for in the kinematic measurements (Table 4). According to Shaw et al. (2009), these inaccuracies could be a function of bone-kinematic instrumentation hardware rigidity. Therefore, mounting of instruments during specimen preparation should be improved to increase rigidity, especially on smaller bones such as the talus, navicular, and calcaneus where differences among 
repeated trial angular and linear displacements were high (Table 4). Also, according to Shaw et al. (2009), these inaccuracies could be a function of the optoelectronic system used to measure the bone kinematics. Instead of the ROMER Arm scans used in this proof-of-concept study, which only measured the initial and final positions of legs before and after applied rotation, a robust system such as the optoelectronic stereo photogrammetric system suggested and assessed, for inaccuracies, by Lessley et al. (2011) should be implemented. This system can measure bonekinematics throughout rotation from start of test to end of rotation, which would be informative when interpreting relative bone motion to documented injuries post-test. Finally, the inaccuracies detailed in Table 4, could also be a function of the compliance in the test fixture and potting materials. Since all bone motions are reported relative to the tibia, rigidity of the proximal tibia is essential, such that tibia compliance and inaccuracies propagate into the kinematics measured for the other foot/ankle bones. These proposed changes to kinematics measurement techniques and improvements to boundary conditions should be implemented in any future tests aimed at recreating syndesmotic ankle injury. These changes should limit inaccuracies and improve the reliability of bone kinematic measurements.

This small-scale, proof-of-concept study was limited by testing a small number of specimens. Despite the limitations and noted inaccuracies (Table 4 and Table 5), a baseline for future studies has been developed. An initial investigation of ankle posture effects on cadaveric leg kinematic responses was established. Relative bone kinematics, in a geometrically defined coordinate system, were measured, e.g. quantified the bounds of bone-specific ankle kinematics within a noninjurious ER and IR regime among varying initial ankle postures. These relative bone kinematics will allow for interpretation of ligament elongation, especially between the tibia and fibula which is essential to further understand ankle mechanics during syndesmotic ankle sprains. Similar kinematics measurement techniques and coordinate system definitions should be implemented in future experiments aimed at re-creating syndesmotic injury during applied foot rotation. The noted necessary improvements to kinematic hardware instrumentation, test fixture, and kinematic measurement methodologies, must be accounted for in these injury tests. Thus, further work, utilizing similar realistic and functionally relevant boundary conditions, as detailed in this chapter, will be (Chapter 3) completed to investigate the effects of applied foot rotation on ankle injuries. 


\section{Chapter 3: Syndesmotic Injury Incidence and Effects of Ankle Flexion Posture}

\subsection{Introduction}

In natural joint motion, external and internal rotation of the foot occurs about a moving axis (Funk, 2011; Rastegar et al., 1980). Restricting this joint motion to artificial, fixed axes of rotation can potentially result in ankle injury patterns and bone kinematics which are not representative of what would occur naturally during forceful external foot rotation. Mait et al. (2015, 2017b) developed an experimental method (Section 2.2) to apply external foot rotation in cadaver legs without artificially restricting the motion of the foot or fibula. This method, from the knowledge gained in Chapter 2, was adapted in this chapter and applied to injurious, hyper-external foot rotation experiments.

Though the main injury mechanism is well-documented, the sequence and propagation of injury among the ankle ligaments during external foot rotation, and their sensitivity to ankle orientation in other planes of motion, remains unclear (Mait et al., 2017a). A more complete understanding of syndesmotic injury can be attained by investigating effects of initial ankle flexion posture on this injury sequence. To accurately re-create syndesmotic injury, ligamentous injury must be present (Mait et al., 2017a). Older cadavers (Table 1) were tested in previous studies (Markolf et al., 1989; Michelson et al., 1997), which resulted in a high incidence of fibula fractures, but others tested younger cadavers resulting in higher incidences of ligamentous injury (Villwock et al., 2009; Wei et al., 2012b, 2010). Though testing younger cadavers is important for proper syndesmotic injury re-creation, a complete diagnosis of all injuries throughout the applied foot rotation cycle may be more important. Syndesmotic ankle sprains are complicated injuries involving multiple anatomic structures (Anderson et al., 2010; Williams et al., 2007). Previously, a single injury at failure, whether ligamentous or bony, was reported (Markolf et al., 1989; Michelson et al., 1997; Villwock et al., 2009; Wei et al., 2012b, 2010). However, this does not accurately describe syndesmotic injury re-creation, and thus a clinically significant propagation and sequence of injury must be ascertained post-test. By observing the sequence of injury, propagation of injury through the 
structures of the ankle may be understood, thus informing efforts for injury prediction (e.g., knowing which ligament is injured first) and prevention.

Expanding on the knowledge gained from the work detailed in Chapter 2, the work in this chapter aimed to apply functionally realistic boundary conditions during an injurious, forceful external foot rotation loading. Using these functionally relevant boundary conditions, an objective of this chapter was to re-create syndesmotic ankle sprains in non-senescent, sizable cadaveric legs (Objective 2). From this re-creation, ankle injury incidences and a consistent injury sequence for the proposed injury mechanism, external foot rotation, in cadaveric limbs oriented in an initially neutral ankle posture (Objective 2), can be determined. Also, the work in this chapter intended to evaluate how initial ankle flexion posture (e.g., initial dorsiflexion and plantarflexion) modifies the incidence and sequence of syndesmotic injury (Objective 3). Lastly, the work in this chapter aimed to relate the injury timing among ankle anatomic structures in all initial ankle postures to the ankle bone kinematics measured during experimentation and quantified using the rigorously defined coordinate systems detailed in Chapter 2.

\subsection{Methods}

\subsubsection{Specimen Preparation}

Experiments were conducted on nine matched-pairs of fresh-frozen male cadaver legs (Table 6) in varying initial ankle flexion postures: neutral $(n=9)$, plantarflexed $(n=4)$, and dorsiflexed $(n=$ 4). Non-senescent, sizable cadaver legs were acquired with intention of targeting the average height, weight, and age of professional American football players (Manfred, 2014) in the National Football League (NFL). Specimens were acquired with the approval of and prepared in accordance with the policies and procedures of the UVA Center for Applied Biomechanics Oversight Committee (Ethics Approval \#: CAB2014-07). All specimens were confirmed free of infectious diseases, including HIV and Hepatitis $\mathrm{B} / \mathrm{C}$, stored at $-15^{\circ} \mathrm{C}$, and thawed at room temperature for 48 hours prior to test preparation.

To prepare specimens for testing, as in Section 2.2.1, the tibia and fibula were disarticulated at the knee. Soft tissue surrounding the tibial plateau and the anterior, medial, and posterior portions of 
the tibia shaft from the tibial plateau to approximately $10 \mathrm{~cm}$ distal was removed. To not disturb proximal tibiofibular ligaments and IOM, superficial soft tissue around the fibula was left intact (Mait et al., 2017b, 2017a). However, to improve the rigidity of the tibia potting and ensure better alignment of the tibia in the test fixture (noted deficiencies in the proof-of-concept study in Chapter 2), the medial and lateral intercondylar eminences were shaved off to flatten the tibial plateau. This allowed the potting cup top to be flush with the tibial plateau, improving the alignment of the tibia's long-axis with the global compression and rotation axis (detailed below, Section 3.2.2). This was done in an attempt to limit the inaccuracy of tibia kinematics measurement which should improve the reliability of the kinematics measured for all other foot and ankle bones.

Table 6: Specimen whole-body anthropometric data with corresponding test input conditions. Cadaver anthropometric data is compared to that the NFL average player in 2014 (Manfred,

\begin{tabular}{|c|c|c|c|c|c|c|c|}
\hline Run \#* & Specimen $\#^{* *}$ & Gender & Age (yr.) & $\begin{array}{l}\text { Height } \\
(\mathrm{cm})\end{array}$ & $\begin{array}{c}\text { Weight } \\
(\mathrm{kg})\end{array}$ & $\begin{array}{c}\text { Preload } \\
(\mathrm{kN})\end{array}$ & $\begin{array}{l}\text { Input Rotation } \\
\text { Magnitude }\left({ }^{\circ}\right)\end{array}$ \\
\hline 1 & $696 \mathrm{R}$ & Male & 49 & 175.3 & 61.2 & 2 & 60 \\
\hline 2,10 & $680 \mathrm{~L}, \mathrm{R}$ & Male & 58 & 175.3 & 63.0 & 2 & 53,90 \\
\hline 3,14 & $682 \mathrm{~L}, \mathrm{R}$ & Male & 54 & 183.0 & 57.1 & 2 & 60,90 \\
\hline 4,12 & $615 \mathrm{~L}, \mathrm{R}$ & Male & 57 & 183.0 & 94.3 & 2 & 90,90 \\
\hline 5,13 & 794L, R & Male & 57 & 177.8 & 149.2 & 2 & 90,90 \\
\hline 6,17 & $801 \mathrm{~L}, \mathrm{R}$ & Male & 36 & 173.0 & 92.0 & 2 & 90,90 \\
\hline 7,11 & $612 \mathrm{~L}, \mathrm{R}$ & Male & 36 & 167.5 & 113.4 & 2 & 90,90 \\
\hline 8,15 & 752L, R & Male & 27 & 182.9 & 97.1 & 2 & 90,90 \\
\hline 9,16 & 757L, R & Male & 49 & 185.4 & 122.0 & 2 & 90,90 \\
\hline & $\begin{array}{c}\text { Average } \\
\text { (9 Specimens) } \\
\end{array}$ & & 47 & 178.1 & 94.4 & & \\
\hline & NFL Average & & 27 & 188.1 & 113.6 & & \\
\hline
\end{tabular}

* Specimens in Runs 1 through 9 were Neutral, 10 through 13 Plantarflexed, and 14 through 17 Dorsiflexed

**L indicates left lower limb specimen, tested first, and $\mathrm{R}$ indicates the matched-pair right lower limb specimen, tested second.

As in Section 2.2.1, a potting cup was rigidly fixed to the proximal tibia using wood screws across the tibial plateau and at varying heights and angles around the proximal tibia shaft (Mait et al., 2017a, 2017b). The center of the potting cup was coincident with the center of the tibial plateau. To increase potting rigidity, threaded rods were drilled from anterior to posterior through the proximal tibia and sunk into a plastic insert on the posterior side of the potting cup. Bondo body 
filler (part \#261, 3M Company, St. Paul, MN, USA) surrounded the tibial plateau to ensure a rigid connection between the tibia and potting cup (Figure 17). The anterior-posterior threaded rods through the proximal tibia were used to improve rigidity of the tibia in the test fixture, in an attempt to improve tibia kinematic measurements and subsequent reliability of other ankle/foot bone kinematics.

To complete specimen preparation, tri-axial rosette strain gauges (Micro-Measurements, Vishay Precision Group, Inc., Wendell, NC, USA) were glued to the surfaces of the tibia and fibula (distal third) of the legs and marker sets for measuring kinematics were rigidly affixed to the tibia, fibula, talus, calcaneus, navicular, $5^{\text {th }}$ metatarsal, and $2^{\text {nd }} / 3^{\text {rd }}$ metatarsal bones (Figure 17). Each marker set, considered the 'marker cluster' (Cappozzo et al., 1997; Shaw et al., 2009), contained four reflective, spherical markers attached to an aluminum plate with threaded rods. Wood screws passed through these plates and drilled into each bone. The kinematic marker set plates had countersunk holes on their superior face (Figure 17). To rigidly fix the marker set to each bone, screws passed through aluminum standoffs, which were flush between the bone surface and the inferior side of the plate (Mait et al., 2017a), and tapped into the bone. This allowed for the screws to be tightened into the countersunk holes as the standoffs laid flush with the bone surface, thus keeping a consistent distance standoff distance for each marker set from the bone so that soft tissue did not directly interact with the markers. This design for the kinematic instrumentation hardware was engineered to address the deficiencies of marker hardware rigidity observed in Chapter 2.
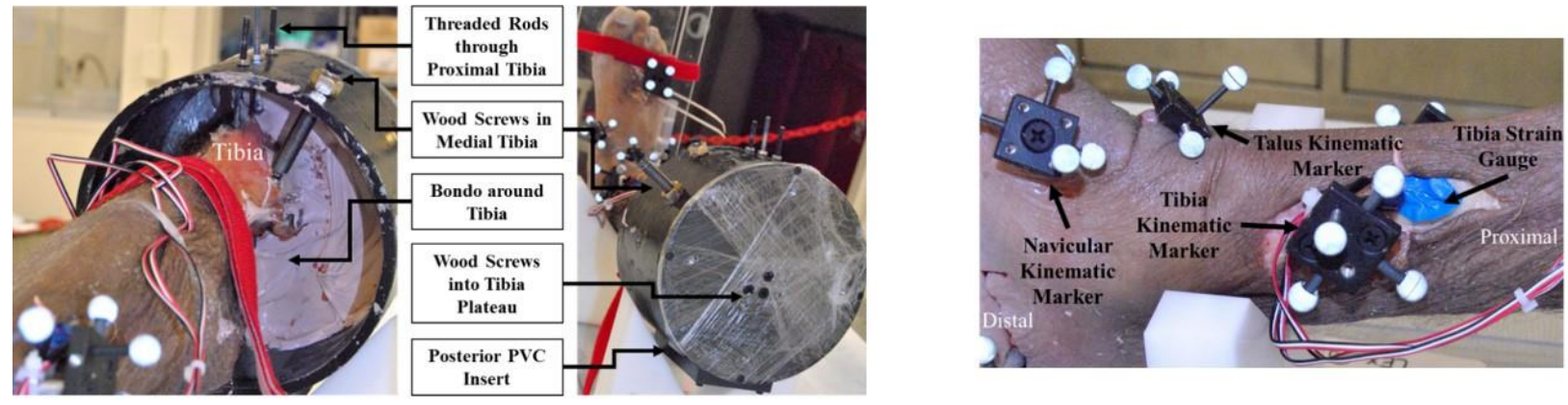

Figure 17: Specimen preparation photos indicating the potting method (left) and kinematic marker placements in the ankle bones and strain gauge attachment on the tibia (right).

CT scans were taken after specimen instrumentation to document the locations of the marker sets relative to each bone and to confirm the absence of bony trauma. A multi-camera, optoelectronic 
stereo photogrammetric motion capture system (Hardware: Vicon T-series; Software: Nexus 2.2; Vicon, CA, USA) was used to measure the three-dimensional motion of each bone in the laboratory (global) space. The underlying motion of each bone was calculated from the measured motion of each marker set using a rigid-body transformation analysis (Lessley et al., 2011; Shaw et al., 2009), as detailed in Sections 2.2.2 and 2.2.3. This optoelectronic stereo photogrammetric motion capture system has been suggested, and furthermore assessed, previously by Lessley et al. (2011) as a sufficient system to reconstruct the movement of bony anatomical landmarks, no matter if the trajectory of these landmarks are observable experimentally or not (Cappozzo et al., 1997). According to Lessley et al. (2011), deviations between actual measured bone center positions and the calculated bone center positions from the optoelectronic photogrammetric motion capture system were sub-millimeter on average. Therefore, this motion capture system was adopted for the current study with the intention of minimizing inaccuracies, as noted in Chapter 2, in bone kinematics measurement experimentally. Additionally, this motion capture system allows for kinematics measurement throughout a continuously applied rotation cycle instead of the initial and final position three-dimensional scans used in Chapter 2. Kinematic measurements throughout the rotation cycle will be informative when interpreting the relationship between injuries induced during the experiments and relative bone motion. Inherent to all experiments are inaccuracies in data measurement, however the work by Lessley et al. (2011) instilled confidence in the experimenters that this motion capture system would be sufficient to measure the underlying motion of the foot and ankle bones during experimentation. Therefore, this motion capture system measures bone kinematic data reliably, throughout the applied rotation cycle and during induced injuries, in a coordinate system convention that is rigorously defined using bone geometry and is interpretable in clinically relevant ankle rotation terms.

For an overall description of kinematics during the test, motion of all bones were analyzed relative to the global $\mathrm{X}-\mathrm{Y}-\mathrm{Z}$ coordinate system: Z-axis positive distally and coincident with tibia long-axis, $\mathrm{X}$-axis positive anteriorly and parallel to the toes, and $\mathrm{Y}$-axis positive medially for left legs and laterally for right legs (Mait et al., 2017a). Motion of the fibula, calcaneus, talus, navicular, $5^{\text {th }}$ metatarsal, and $2^{\text {nd }} / 3^{\text {rd }}$ metatarsals were also analyzed relative to the local coordinate system of the tibia. Based on the geometric coordinate system methodologies detailed in Section 2.2.2, the tibia coordinate system was defined with the z-axis along the long-axis of the tibia (positive distally), 
the $\mathrm{x}$-axis positive anteriorly (parallel to the toes), and the $\mathrm{y}$-axis positive medially for left legs but laterally for right legs (Mane, 2016). Coordinate systems for the calcaneus, talus, navicular, $5^{\text {th }}$ metatarsal, and $2^{\text {nd }} / 3^{\text {rd }}$ metatarsal bones were defined initially at the centroid of each bone with all axes parallel to that of the tibia coordinate system in a neutral posture (Mait et al., 2017b, 2017a; Mane, 2016). Similarly, the initial coordinate axes for the fibula were defined at the centroid of the lateral malleolus. Again, the neutral posture (Section 2.2.1) was defined nominally such that a right angle formed between the distal end of the first phalanx, calcaneus centroid, and long-axis of the tibia in the sagittal plane (Mait et al., 2017a).

Using the geometric coordinate system definitions (Section 2.2.2) and rigid-body transformation analyses (Shaw et al., 2009), the three-dimensional changes in position and orientation (linear and angular displacements) of each bone from the neutral posture to a non-neutral posture were described as three Euler angles ( $\Delta$ yaw, $\Delta$ pitch, $\Delta$ roll) in the z-y-x convention and three linear displacements $(\Delta \mathrm{x}, \Delta \mathrm{y}, \Delta \mathrm{z})$ along the axes of the tibia (Sections 2.2.2 and 2.2.3). These changes in position and orientation from the neutral posture were calculated throughout the rotation cycle. Subsequently, external foot rotation (ER), induced by rotating the tibia internally, was defined as the axial rotation $(\Delta y a w)$ of the calcaneus relative to the long-axis of the tibia in the transverse plane.

\subsubsection{Test Apparatus and Loading Protocol}

Prior to testing, specimens were preconditioned by manually rotating the feet within physiological ranges of motion for ankle external-internal rotation, eversion-inversion, and dorsiflexionplantarflexion (Nigg et al., 1990; Roaas and Andersson, 1982). Specimens were tested in an Instron bi-axial testing machine (Axial-Torsion Servohydraulic Fatigue Testing System, FastTrack 8800 Materials Test Control System, Instron Corporation, Norwood, MA, USA). The proximal tibia potting cup was rigidly attached to the Instron piston head (Figure 18 and Figure 19) through a 6-axis load cell (Model \#3868TF, Denton ATD, Inc., Rochester Hills, MI, USA). Through its linear actuator, the Instron imparted the desired preload along the global Z-axis (defined initially coincident with the long-axis of the tibia) and effective gross external foot rotation by rotating the tibia internally about the global Z-axis. For testing, all specimens were subjected to a compressive preload $\left(\mathrm{F}_{\mathrm{Z}}\right)$ targeting $2 \mathrm{kN}$ (Table 6 ) along the global Z-axis, chosen to simulate weight-bearing 
during typical play situations (Wei et al., 2012a, 2010). Through its rotary actuator, the Instron applied the external rotation in a half-sine waveform. Rotation frequency was $0.05 \mathrm{~Hz}\left(18^{\circ} / \mathrm{s}\right)$ for Run 1 and $0.025 \mathrm{~Hz}\left(9^{\circ} / \mathrm{s}\right)$ for all subsequent runs. Rotation magnitudes were varied, seeking to find a magnitude that would consistently result in injury (Table 6). Low rotation rates were chosen to limit inertial effects and be within physiological range (Wei et al., 2010) during testing, and were decreased after Run 1 to limit binding in the test fixture.

Kinetic, kinematic, and audio data were measured during testing for all specimens (Table 7). Load cells measured forces and moments at the proximal tibia, hindfoot, and forefoot (see Sections 3.2.3 and 3.2.4 below for more details). Strain gauges on the tibia and fibula were used to measure strain changes. An external microphone was placed next to the test area to capture audible pops, indicating injury with sufficient energy release to generate a measurable audio signal. A bonemounted acoustic emission sensor was glued to the surface of the fibula in an effort to record the timing of more subtle ligament injuries. Though not shown in Table 7, the instrumentation for Runs 10-17 was the same as that used for Runs 7-9.

Table 7: Summary of instrumentation data channels measured in all Neutral Tests. Note: the instrumentation used in Runs 7-9 were repeated for the tests in initial plantarflexion (Runs 10-13) and dorsiflexion (Runs 14-17).

\begin{tabular}{ccccccccccc}
\hline & Run \# & 1 & 2 & 3 & 4 & 5 & 6 & 7 & 8 & 9 \\
\cline { 2 - 10 } Instrumentation & Specimen \# & $696 \mathrm{R}$ & $680 \mathrm{~L}$ & $682 \mathrm{~L}$ & $615 \mathrm{~L}$ & $794 \mathrm{~L}$ & $801 \mathrm{~L}$ & $612 \mathrm{~L}$ & $752 \mathrm{~L}$ & $757 \mathrm{~L}$ \\
\hline \multirow{2}{*}{ Tibia LC } & Force-x,y,z & $\checkmark$ & $\checkmark$ & $\checkmark$ & $\checkmark$ & $\checkmark$ & $\checkmark$ & $\checkmark$ & $\checkmark$ & $\checkmark$ \\
& Moment-x,y,z & $\checkmark$ & $\checkmark$ & $\checkmark$ & $\checkmark$ & $\checkmark$ & $\checkmark$ & $\checkmark$ & $\checkmark$ & $\checkmark$ \\
\hline \multirow{2}{*}{ Hindfoot LC } & Force-x,y,z & & & & $\checkmark$ & $\checkmark$ & $\checkmark$ & $\checkmark$ & $\checkmark$ & $\checkmark$ \\
& Moment-x,y,z & & & & & & $\checkmark$ & $\checkmark$ & $\checkmark$ \\
\hline \multirow{2}{*}{ Forefoot LC } & Force-x,y,z & & & $\checkmark$ & $\checkmark$ & $\checkmark$ & $\checkmark$ & $\checkmark$ & $\checkmark$ \\
& Moment-x,y,z & & & $\checkmark$ & $\checkmark$ & $\checkmark$ & $\checkmark$ & $\checkmark$ & $\checkmark$ \\
\hline \multirow{2}{*}{ Strain Gauges } & Tibia-1,2,3 & $\checkmark$ & $\checkmark$ & & $\checkmark$ & $\checkmark$ & $\checkmark$ & $\checkmark$ & $\checkmark$ \\
& Fibula-1,2,3 & $\checkmark$ & $\checkmark$ & & $\checkmark$ & $\checkmark$ & $\checkmark$ & $\checkmark$ & $\checkmark$ \\
\hline Acoustic & & & & & & & & & $\checkmark$ \\
Emission & & & & $\checkmark$ & $\checkmark$ & & $\checkmark$ & $\checkmark$ & $\checkmark$ \\
\hline External & & & & $\checkmark$ & $\checkmark$ & $\checkmark$ & $\checkmark$ & $\checkmark$ & $\checkmark$ \\
Microphone & & & & & & & & & \\
\hline
\end{tabular}

Note. Instrumentation included 6-axis (3 forces and 3 moments) and 3-axis (3 forces) load cells, triaxial rosette strain gauges, bone-mounted microphone (acoustic emission sensor), and external microphone. 


\subsubsection{Pilot Study: Adapting Quasi-Static Experimental Boundary Conditions}

As an initial pilot study for the injurious tests, a subset ( $n=3$, Runs 1 through 3 ) of the initially neutral posture legs were tested with boundary conditions adapted from Chapter 2 (Section 2.2.4) and Mait et al. (2015, 2017b). This was done to further confirm the validity of the improved, functionally relevant boundary conditions in an injurious, dynamic scenario.

To summarize from Section 2.2.4, the proximal tibia was fixed in a potting cup with the fibula unconstrained. The calcaneus was constrained from rotating and translating relative to other bones by placing a mound around the calcaneus, assembled with acetal homopolymer resin and polyvinyl chloride and reinforced with machine screws. A bi-directional linear rail system was rigidly attached to a gimbal system which locked the foot in a neutral posture (Figure 18, top). The calcaneus mount was rigidly fixed to the gimbal system, thus permitting foot translation in the global X (anterior-posterior) and Y (medial-lateral) directions and allowing the axis of external rotation to adjust to the natural axis of rotation in the lower limb (Funk, 2011; Mait et al., 2017b; Rastegar et al., 1980). However, to address the inaccuracies noted in Section 2.3 for kinematic measurements, the boundary conditions in this pilot study were slightly modified. As detailed in Section 3.2.1, rigidity of the tibia potting to the test fixture was improved by shaving off the tibia intercondylar eminences and sinking threaded rods from anterior to posterior in the proximal tibia. To improve calcaneus rigidity to the test fixture, and calcaneus kinematics measurement, Bondo body filler was applied inside the calcaneus mount. All soft tissue, including skin, remained intact around the calcaneus such that the Bondo did not directly influence ligamentous connections. These changes from the quasi-static boundary conditions were made to improve the fixation of the specimen to the test fixture in preparation for the injurious, dynamic rotational loading where compressive preload was increased and higher magnitudes of reaction kinetics in the leg were expected.

In this pilot study of injurious tests (Runs 1-3), the Instron rotated the tibia internally over the locally fixed calcaneus, which was free to translate grossly, thus imparting the external foot rotation. Boundary conditions (Figure 18, top) consisted of one force ( $F_{Z}$ preload) and three 
moments ( $\mathrm{M}_{\mathrm{X}}$ reaction at calcaneus, $\mathrm{M}_{\mathrm{Y}}$ reaction at calcaneus, $\mathrm{M}_{\mathrm{Z}}$ input rotation torque). These boundary conditions, with the calcaneus in a fixed orientation, are referred to, in Table 8, as Boundary Condition B (BC:B). The boundary conditions, without the improvements listed above for this injurious pilot study, utilized in the quasi-static proof-of-concept study (Section 2.2.4) are referred to as Boundary Condition A (BC:A) in Table 8. 


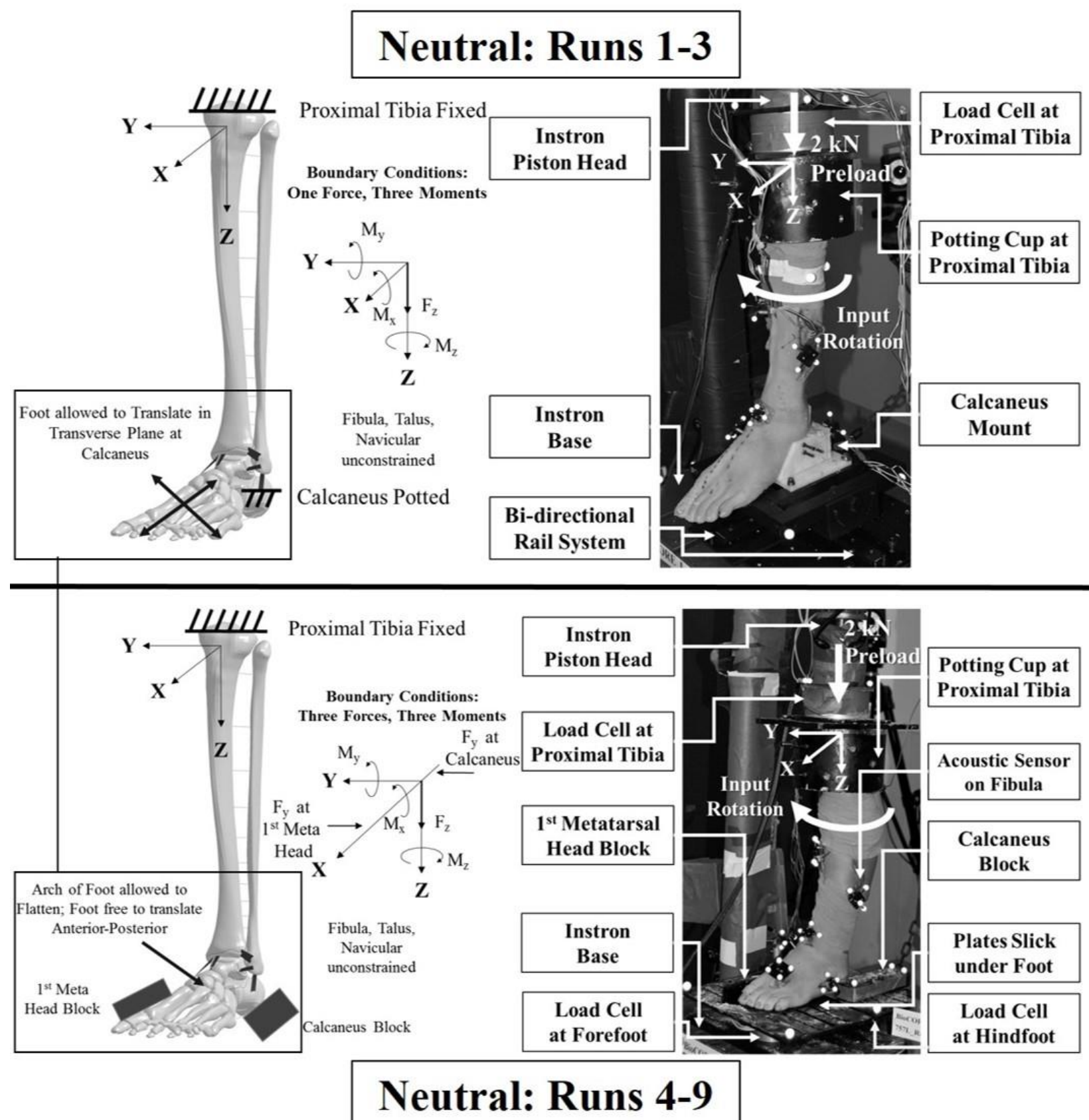

Figure 18: Initially neutral posture specimen test apparatus and corresponding boundary condition schematics for the $\mathrm{BC}: \mathrm{B}$ (calcaneus in fixed orientation) test fixture used in Runs 1-3

(top) and the BC:C (calcaneus free to invert/evert) test fixture used in Runs 4-9 (bottom).

$\mathrm{BC}: \mathrm{B}$ represents an improvement, in terms of being more functionally relevant and realistic for applied foot rotation, compared to previous experiments (Markolf et al., 1989; Michelson et al., 1997; Villwock et al., 2009; Wei et al., 2012b, 2010; Xenos et al., 1995), where the tibia and fibula are rigidly coupled and foot translation is not permitted (Table 1). However, some noted 
deficiencies were found by the experimenters during testing which needed to be addressed in further injurious tests after this pilot study (Runs 1-3). With the bi-directional rail system, alignment of the tibia was challenging for one of the specimens such that its initial position was more of an artificially plantarflexed posture and not completely neutral (Figure 19). The rail system, even though it permits foot translation during rotation, constricts the foot to translate grossly in two predefined directions. Additionally, the calcaneus mount and gimbal did not engage the entire foot during rotation such that the talus and foot (midfoot and anterior) were allowed to droop (Figure 19).

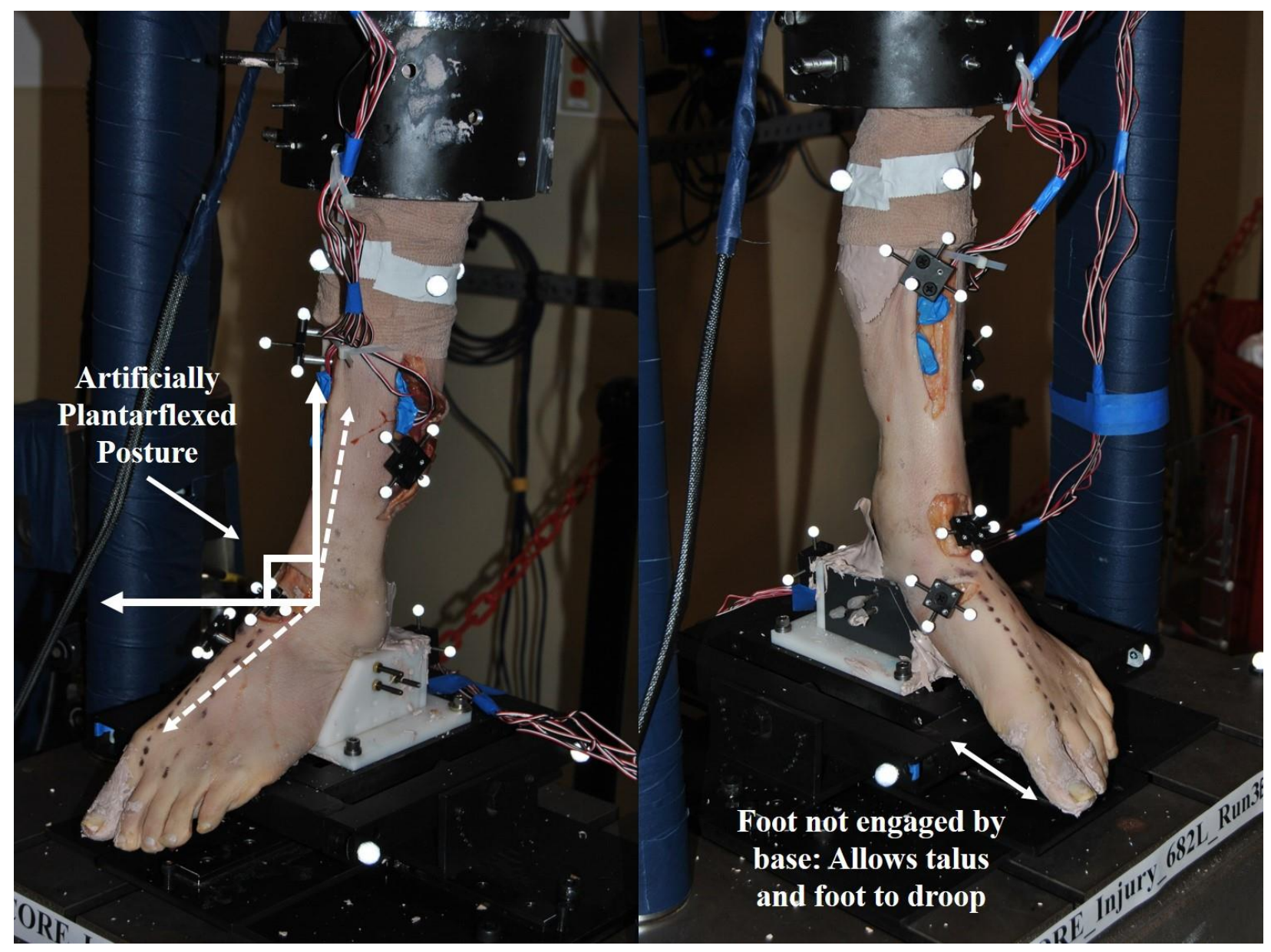

Figure 19: Specimen in the test fixture for the injurious pilot study (Runs 1-3) with BC:B where the leg was positioned in an artificially plantarflexed posture (left). The foot was not engaged by the test fixture in $\mathrm{BC}$ : $\mathrm{B}$ during applied rotation, thus allowing the talus and foot to droop (right).

The boundary conditions implemented in this injury pilot study (BC:B), was an attempt to balance functionally relevant yet mechanically tractable boundary conditions (i.e., experimental 
boundaries are controlled, interpretable, and defined thoroughly, yet still permit functional anatomical motion), with which relevant bony motion can be measured. Compared to previous studies (Markolf et al., 1989; Michelson et al., 1997; Villwock et al., 2009; Wei et al., 2012b, 2010; Xenos et al., 1995), BC:B represents the first concerted effort at identifying a set of external boundary conditions on the leg that impose realistic and injurious loads throughout the ankle, yet are reasonably representative of those that occur in athletes on the field of play. Those previous experiments over-constrained the leg and $\mathrm{BC}: \mathrm{B}$ was less restrictive by permitting leg compliance through an unconstrained fibula and allowable gross foot translation. However, with the artificially plantarflexed posture and talus/foot droop, BC:B imposed a potentially unrealistic loading throughout the leg and foot during applied rotation. Though the natural axis of rotation within the leg was allowed to adjust throughout rotation, through the rail system (calcaneus translation), in both $\mathrm{BC}: \mathrm{A}$ and $\mathrm{BC}: \mathrm{B}$, the entire foot was not engaged during loading. To ensure proper ligament recruitment and therefore a realistic re-creation of syndesmotic injury, the entire foot should be engaged and initial ankle posture should be more well-defined. Simultaneously allowing for controlled loading, functional relevance, and measurement of all applied loads and resulting bony motion in an experiment aimed at re-creating realistic ligamentous injury, as would be expected in athletes, is challenging. However, based on the deficiencies listed above, BC:B creates a somewhat unrealistic re-creation of a syndesmotic injury scenario. Therefore, to address these experimental deficiencies, boundary conditions were adapted for subsequent injury tests to create an experimental protocol which more closely represented the external boundaries expected to be observed on-field in athletes.

\subsubsection{Boundary Condition Improvements}

From the deficiencies in the imposed external boundaries found in the injurious pilot study (above), and to improve realistic syndesmotic injury re-creation, boundary conditions were changed (Figure 18, bottom) for the remainder of tests in the initially neutral ankle flexion posture (Runs 4-9). This change in boundary conditions was intended to mimic feet constrained in a cleat on the football field, where the cleat is planted in the turf and the tibia rotates internally over the constrained foot (Wei et al., 2012a). The test apparatus was designed to allow the foot and calcaneus to undergo natural inversion and eversion during applied external foot rotation. A pair 
of flat plates, orthogonal to the long-axis of the tibia, supported the plantar surface of the foot. These orthogonal plates (to the tibia long-axis) ensured proper neutral ankle posture in the experiments instead of a potentially unrealistic, artificially plantarflexed posture, as seen in the injurious pilot study (Figure 19). Neutral initial ankle posture was maintained by orienting the plantar foot support plates parallel in the transverse (X-Y) plane. To engage the heel and forefoot during foot rotation, lateral and opposing support blocks were placed adjacent to the calcaneus (laterally) and first metatarsal head (medially). This restrained the foot while the tibia was internally rotated by the Instron. All surfaces in contact with the foot were lubricated (Tri-Flow Superior Teflon Chain Lube, The Sherwin-Williams Company, Cleveland, OH, USA) to minimize binding and permit foot arch flattening and gross foot translation in the anterior-posterior direction (Figure 18, bottom).

In addition to the load cell superior to the proximal tibia, six-axis load cells (Model \#s: 6085; 7414, Humanetics Innovative Solutions, Plymouth, MI, USA) were placed under the forefoot and hindfoot (inferior to the plantar foot plates) to measure reaction forces under both portions of the foot (the plantar foot plates were separated by 0.5 inches). Boundary conditions (Figure 18, bottom) consisted of three forces $\left(\mathrm{F}_{Z}\right.$ preload, $\mathrm{F}_{Y}$ reaction at first metatarsal head, FY reaction at calcaneus) and three moments $\left(\mathrm{M}_{\mathrm{X}}\right.$ reaction at forefoot and hindfoot, $\mathrm{M}_{\mathrm{Y}}$ reaction at forefoot and hindfoot, $\mathrm{M}_{\mathrm{Z}}$ input rotation torque). These boundary conditions, with calcaneus inversion and eversion permitted and leg/foot alignment dictated by orthogonal plantar plates to the tibia longaxis, are referred to as Boundary Condition $\mathrm{C}(\mathrm{BC}: \mathrm{C})$ in Table 8. BC:C addresses the deficiencies of $\mathrm{BC}: \mathrm{B}$ detailed above in the injurious pilot study such that a more realistic and functionally relevant representation of the external boundaries experienced by athletes on-field was implemented. The entire foot is engaged, permitting proper bony motion in the foot/ankle and subsequent ligament recruitment, and gross foot translation is still permitted to allow the natural axis of rotation in the leg to adjust during applied rotation (Funk, 2011; Rastegar et al., 1980).

With a set of external boundary conditions (BC:C, Runs 4-9) that mimic feet constrained in a cleat on the football field now established in the neutral ankle flexion posture, changes in these boundary conditions to accommodate varying initial ankle flexion postures were considered. Initial ankle plantarflexion of $30^{\circ}$ and dorsiflexion of $15^{\circ}$ were desired. As in $\mathrm{BC}$ :C, tibia potting and alignment 
was the same as was the boundaries surrounding the foot (plantar plates inferior to the foot and lubricated blocks constraining the calcaneus and first metatarsal head) in the tests on the initially plantarflexed (Runs 10-13) and dorsiflexed legs (Runs 14-17). To impart the desired degrees of ankle flexion, a wedge was rigidly fixed to the plantar foot plates: $30^{\circ}$ and $15^{\circ}$ wedges were used for plantarflexion (Figure 20, top) and dorsiflexion (Figure 20, bottom). To prevent the specimens from slipping down the inclined plates under the preload, a block was placed anterior to the toes in plantarflexion and posterior to the calcaneus in dorsiflexion. This restrained anterior-posterior foot translation; however, the natural axis of rotation of the tibia was still able to adjust throughout the rotation cycle, since all surfaces in contact with the foot were lubricated (including the blocks anterior to the toes, if plantarflexion, and posterior to the calcaneus, if dorsiflexion). This permitted the calcaneus to invert and evert freely (same as in BC:C) and permitted foot arch flattening and gross foot bone motion between the foot block constraints. Boundary conditions for these changes in ankle flexion posture are detailed in Figure 20, with boundary conditions in the initially plantarflexed ankle posture referred to as Boundary Condition D (BC:D) and in the dorsiflexed posture as Boundary Condition E (BC:E) in Table 8. The right limbs from a matched pair were tested in initial plantar- or dorsiflexion (Table 6), with the left limbs tested in neutral (either BC:B or BC:C). One exception was for Run 1 (Specimen 686R) tested in BC:B which did not have a matched pair limb in either of the initial plantar- or dorsiflexion tests. 


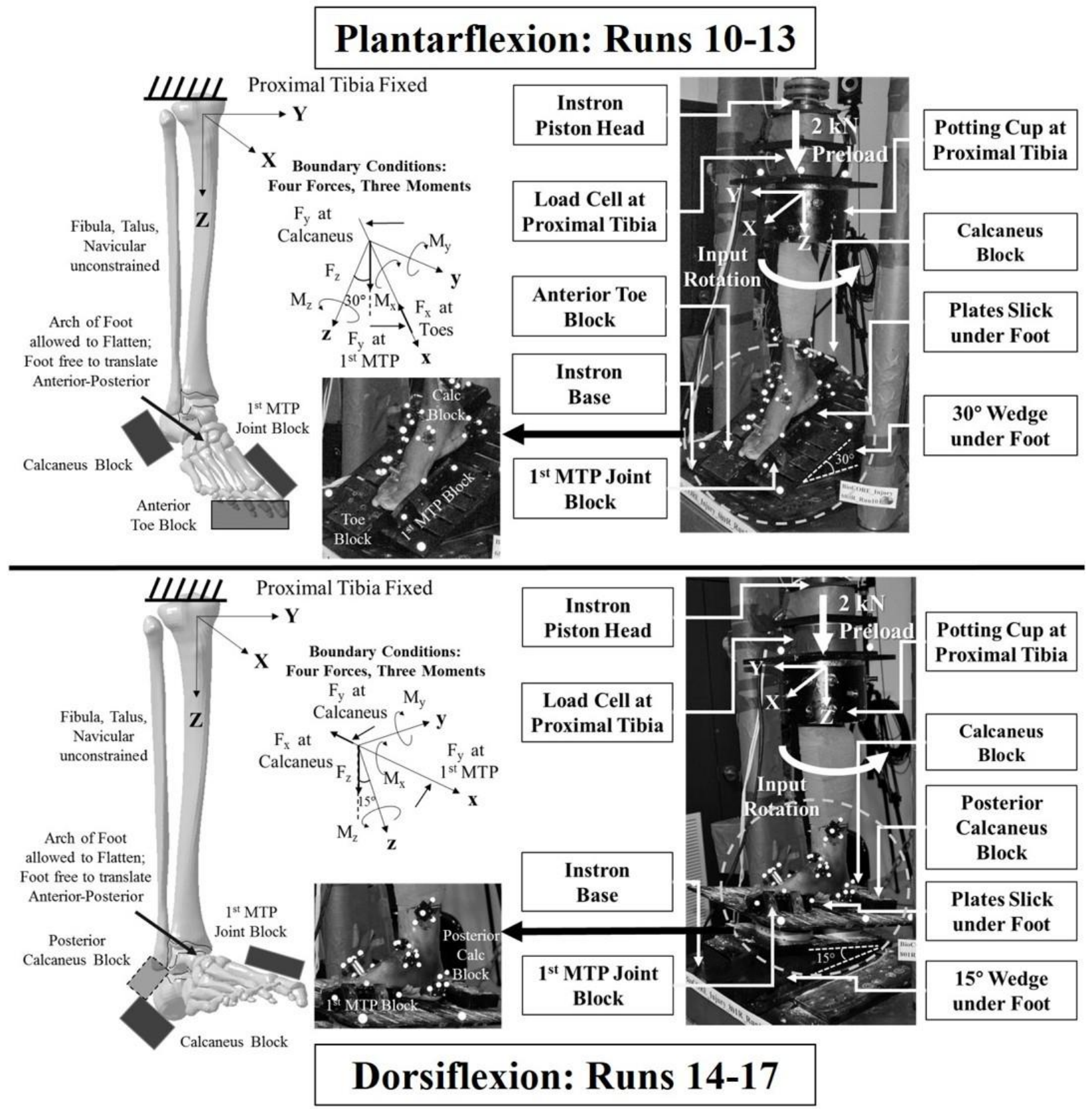

Figure 20: Specimen test apparatus and corresponding boundary condition schematics for the initially plantarflexed (BC:D, Runs 10-13) ankle posture tests (top) and initially dorsiflexed (BC:E, Runs 14-17) ankle posture tests (bottom).

To conceptualize the evolution of the external boundary conditions imposed on the legs in all the experiments discussed thus far in this chapter and Chapter 2, Table 8 details each iteration of the boundary conditions for improvements to specimen preparation and the respective test fixtures from the quasi-static, proof-of-concept tests to the injury tests in initially plantar- and dorsiflexed 
ankle postures. The boundary conditions implemented in Runs 4-9 (BC:C), Runs 10-13 (BC:D), and Runs 14-17 (BC:E) are a result of several iterations, with accompanying incremental improvements, made to the external boundary conditions imposed on the leg during applied foot rotation experiments. These iterations were made in an attempt to balance the need for functionally relevant yet mechanically tractable boundary conditions imposed on the leg. Little work has been done previously, for applied foot rotation experiments, which explicitly identifies a set of boundary conditions designed to impose reasonable and injurious loads throughout the ankle joint while simultaneously allowing for measurement of all applied loads and resulting relative bony kinematics. $\mathrm{BC}: \mathrm{C}, \mathrm{BC}: \mathrm{D}$, and $\mathrm{BC}: \mathrm{E}$ are reasonably representative, compared to the boundary conditions used in previous studies, of the external boundaries imposed on an athlete's leg during a syndesmotic injury scenario on-field by mimicking feet constrained in a cleat on the football field (Wei et al., 2012a). The evolution of boundary conditions from BC:A to BC:E (Table 8) encompass the efforts made to improve applied foot rotation experiments from the overlyconstrained legs in previous studies to a realistic re-creation of syndesmotic injury in cadaveric legs, from which reliable bone kinematics can be measured. Rigidly coupling the tibia and fibula and preventing foot translation certainly over-constrains the leg (Table 1) and is deficient for realistically re-creating syndesmotic injury. Fixing the tibia only and permitting fibula compliance (BC:A through BC:E) addresses these deficiencies in part. Permitting gross foot translation through the calcaneus along a predefined set of axes (BC:A and BC:B) addresses these deficiencies, again in part. However, BC:A and BC:B still artificially constrain the leg, partially, by imparting foot rotation about a calcaneus constrained from rotating naturally. Allowing the calcaneus (and foot) to invert and evert naturally during applied foot rotation, with fibula compliance and gross foot translation still permitted (BC:C, BC:D, and $\mathrm{BC}: \mathrm{E})$, more closely mimics a loading experienced by athletes during play. However, with the added compliance in the calcaneus and foot, interactions between test fixture and bone instrumentation is possible. Regardless of imposed boundary condition choice, tradeoffs are inherent and must be acknowledged. Despite the tradeoffs, BC:C, BC:D, and BC:E represent (in multiple ankle flexion postures) the closest representation of boundary conditions, compared to previous studies, imposed on athlete's during play while still being functionally relevant and mechanically tractable, such that resulting bone kinematics can be measured during syndesmotic injury re-creation. 
Table 8: Summary of boundary condition evolution used in applied foot rotation experiments. Iterations of boundary condition changes are detailed from the quasi-static tests to injury tests in various initial ankle flexion postures.

\begin{tabular}{|c|c|c|c|c|}
\hline $\begin{array}{l}\text { Test } \\
\text { Series }\end{array}$ & $\begin{array}{l}\text { Boundary } \\
\text { Condition } \\
\text { Designation }\end{array}$ & $\begin{array}{l}\text { Initial } \\
\text { Ankle } \\
\text { Posture* }\end{array}$ & $\begin{array}{l}\text { Bone Fixation } \\
\text { Boundaries }\end{array}$ & $\begin{array}{c}\text { External Test Fixture Boundaries on } \\
\text { Legs }\end{array}$ \\
\hline $\begin{array}{l}\text { Quasi- } \\
\text { Static }\end{array}$ & BC:A & $\begin{array}{l}\text { Neutral, } \\
\text { EV, IV, DF, } \\
\text { and PF }\end{array}$ & $\begin{array}{l}\text { Fibula, talus, navicular } \\
\text { unconstrained; } \\
\text { Proximal tibia rigid; } \\
\text { Calcaneus fixed in rotation }\end{array}$ & $\begin{array}{l}\text { Gross (global) translation of foot (through } \\
\text { calcaneus) permitted in all directions; } \\
\text { Gimbal used to place legs in varying ankle } \\
\text { postures }\end{array}$ \\
\hline $\begin{array}{l}\text { Injury, } \\
\text { Runs } \\
1-3\end{array}$ & $\mathrm{BC}: \mathrm{B}$ & Neutral & $\begin{array}{l}\text { Fibula, talus, navicular } \\
\text { unconstrained; } \\
\text { Tibia rigidity increased; } \\
\text { Calcaneus fixed in rotation } \\
\text { and rigidity increased }\end{array}$ & $\begin{array}{l}\text { Gross (global) translation of foot (through } \\
\text { calcaneus) permitted in X and Y directions; } \\
\text { Gimbal used to place legs in initially neutral } \\
\text { posture }\end{array}$ \\
\hline $\begin{array}{l}\text { Injury, } \\
\text { Runs } \\
4-9\end{array}$ & $B C: C$ & Neutral & $\begin{array}{l}\text { Fibula, talus, navicular } \\
\text { unconstrained; } \\
\text { Tibia rigidity increased; } \\
\text { Calcaneus free to } \\
\text { invert/evert }\end{array}$ & $\begin{array}{l}\text { Plantar plates constrain ankle in neutral posture; } \\
\text { External foot rotation imposed by constraining } \\
\text { lateral calcaneus motion and medial motion of } \\
\text { the first metatarsal head; } \\
\text { Arch of foot allowed to flatten; } \\
\text { Lubricated surfaces permit anterior-posterior } \\
\text { translation of foot; }\end{array}$ \\
\hline $\begin{array}{l}\text { Injury, } \\
\text { Runs } \\
10-13\end{array}$ & $B C: D$ & PF & $\begin{array}{l}\text { Fibula, talus, navicular } \\
\text { unconstrained; } \\
\text { Tibia rigidity increased; } \\
\text { Calcaneus free to } \\
\text { invert/evert }\end{array}$ & $\begin{array}{l}\text { Plantar plates and wedge impose ankle } \\
\text { plantarflexion posture; } \\
\text { External foot rotation imposed by constraining } \\
\text { lateral calcaneus motion and medial motion of } \\
\text { the first metatarsal head; } \\
\text { Arch of foot allowed to flatten; } \\
\text { Lubricated surfaces permit gross foot } \\
\text { translation within fixture constraints; } \\
\text { Block anterior to toes prevents foot from } \\
\text { slipping under preload }\end{array}$ \\
\hline $\begin{array}{l}\text { Injury, } \\
\text { Runs } \\
14-17\end{array}$ & $\mathrm{BC}: \mathrm{E}$ & DF & $\begin{array}{l}\text { Fibula, talus, navicular } \\
\text { unconstrained; } \\
\text { Tibia rigidity increased; } \\
\text { Calcaneus free to } \\
\text { invert/evert }\end{array}$ & $\begin{array}{l}\text { Plantar plates and wedge impose ankle } \\
\text { dorsiflexion posture; } \\
\text { External foot rotation imposed by constraining } \\
\text { lateral calcaneus motion and medial motion of } \\
\text { the first metatarsal head; } \\
\text { Arch of foot allowed to flatten; } \\
\text { Lubricated surfaces permit gross foot } \\
\text { translation within fixture constraints; } \\
\text { Block posterior to calcaneus prevents foot from } \\
\text { slipping under preload }\end{array}$ \\
\hline
\end{tabular}

$* \mathrm{EV}=$ ankle eversion, $\mathrm{IV}=$ ankle inversion, $\mathrm{PF}=$ ankle plantarflexion, $\mathrm{DF}=$ ankle dorsiflexion

\subsubsection{Clinical Evaluation and Injury Event Sequence Determination}

After test completion, post-test CT scans and X-Rays were taken to document any possible bony trauma. Post-test necropsies were performed by a board-certified orthopaedic surgeon, with 
fellowship training in sports medicine, on all specimens to document injuries caused. Necropsies began at the proximal tibia and fibula and moved distally. Soft tissue was carefully removed to expose anatomic structures in the ankle, namely syndesmotic ligaments (ATiFL, PTiFL, and IOM), then medial ligaments (superficial deltoid complex and deep deltoid complex), and finally lateral ligaments (ATaFL, CFL, and PTaFL) (Golanó et al., 2010; Kaumeyer and Malone, 1980). The necropsy was considered the gold standard diagnostic modality.

After post-test necropsies, injury events were determined for all test runs during the rotation loading cycle by a group of biomechanical engineers, each with at least 2 years of experience in the study of lower extremity injury mechanics, with consultation from orthopaedic surgeons. Features of interest in the mechanical response data traces (i.e., reaction moments/forces, strain, audio, and bone kinematics) were identified as possible injury events. Large changes in magnitude of the mechanical responses, especially in signals for strain, audio, and moment about the Z-axis in the tibia load cell, were typically attributed to failure of stout, large ligaments (e.g., deep deltoid complex, ATiFL, and IOM) and bone fractures. Small changes in magnitude of the mechanical responses were typically attributed to failure of small ligaments (e.g., superficial deltoid complex and CFL) (Golanó et al., 2010; Kaumeyer and Malone, 1980; Wolfe et al., 2001).

For example, significant changes in strain on the tibia indicated injuries to the medial and syndesmotic ankle ligaments, whereas changes in strain on the fibula indicated injuries to the lateral and syndesmotic ankle ligaments. Understanding of the strength and stoutness of certain ligaments, e.g. deep deltoid complex relative to the CFL or ATiFL relative to the superficial deltoid complex or IOM relative to the ATaFL (Golanó et al., 2010; Kaumeyer and Malone, 1980; Wolfe et al., 2001), helped further distinguish injury events. A stronger ligament injury was indicated in conjunction with a larger drop in magnitude of force and moment response during testing.

Combining this anatomical understanding with dissection results and study of mechanical response data, and with the assistance of orthopaedic surgeons intimately familiar with foot and ankle anatomy, the experimenters matched measured mechanical response events with injuries identified during the post-test dissections. The strain, audio, and moment/force traces distinguished catastrophic injury events such as bone fractures from the more subtle ligament ruptures or 
avulsions. The bone kinematic data elucidated location differences between injury events for these subtle ligament ruptures where a distinction between medial, lateral, and syndesmotic ligament injuries could be made. With a geometrically defined coordinate system, relative motion of the ankle bones to the tibia was measured, from which ligament elongation was inferred for the injury event distinctions. Motions of the fibula, calcaneus, and talus relative to the tibia were especially important for these distinctions. For example, changes in fibula translation relative to the tibia was indicative for distinguishing syndesmotic ligament injury, whereas changes in the motion of the fibula in conjunction with changes in talus or calcaneus motion was indicative of lateral ligament injury. Similarly, changes in calcaneus motion relative to the tibia, in conjunction with talus motion changes, were indicative of medial ligament injury.

After reviewing all data traces for each test run, the expert panel of biomechanical engineers and orthopaedic surgeons, as objectively as possible, attempted to attribute a single injury event to a single diagnosed injury. However, complete confidence in injury event distinctions is difficult and one injury event was sometimes associated with multiple injuries. The panel's confidence in matching injury events to particular injuries were affected by a number of factors, including the available mechanical response data traces (e.g., no strain data for Runs 1 and 4 or no audio data for Runs 1-3; Table 7), loss of kinematics data due to interference with test hardware (detailed in Section 3.3 below), and accuracy of kinematics data (see Section 3.2.1). To account for these uncertainties, a confidence level, similar to Kent et al. (2014), was assigned to each diagnosed injury matched to an event: unknown, possible, probable, or certain. Some injuries were patently obvious to match (e.g., bone fractures with large drop in moment magnitude combined with burst in audio signals) and were classified as certain. However, others (e.g., differences between small ligament ruptures such as between the superficial deltoid complex and CFL) were difficult to match with complete certainty and were classified as either probable or possible. For each matched injury event, the time at which the injury occurred during testing was determined from the mechanical response data traces. From this temporal data, the corresponding external foot rotation value (calcaneus $\Delta$ yaw relative to the tibia) was mined for each matched injury event. If matching an injury event to a diagnosed injury was not deemed certain, the confidence levels (unknown, possible, probable) were factored into subsequent data analyses by accounting for the range of 
external foot rotation (minimum to maximum for each event) at which a particular injury occurred (see Chapter 4 for more details).

\subsection{Results}

Clinically significant injuries (i.e., ligament tears and avulsions, capsule tears, and bone fractures) were documented following all seventeen tests. These full diagnoses of injuries with their corresponding event in the injury sequence are reported in Table 9 (Neutral posture, Runs 1-3, BC:B), Table 10 (Neutral posture, Runs 4-9, BC:C), Table 11 (Plantarflexed posture, Runs 10-13, BC:D), and Table 12 (Dorsiflexed posture, Runs 14-17).

Table 9: Injury event sequences for initially Neutral tests, Runs 1-3 (BC:B).

\begin{tabular}{|c|c|c|c|}
\hline Run \# & Specimen \# & Event \# & Injury Description \\
\hline \multirow{4}{*}{1} & \multirow{4}{*}{$696 \mathrm{R}$} & 1 & $\begin{array}{l}\text { ATaFL partial avulsion off fibula; Lateral Capsule rupture with avulsion } \\
\text { off talus }\end{array}$ \\
\hline & & 2 & $\mathbf{X}$ ATiFL complete rupture; Anterior IOM avulsion from tibia \\
\hline & & 3 & X Weber B Fibula Fracture \\
\hline & & 4 & Superficial Deltoid Ligament avulsion off tibia \\
\hline \multirow[b]{2}{*}{2} & \multirow[b]{2}{*}{$680 \mathrm{~L}$} & 1 & Superficial Deltoid Ligament avulsion off tibia \\
\hline & & 2 & $\begin{array}{l}\text { X Inferior ATiFL (AiTiFL) complete rupture with small avulsion off } \\
\text { lateral malleolus }\end{array}$ \\
\hline \multirow{4}{*}{3} & \multirow{4}{*}{$682 \mathrm{~L}$} & 1 & Initiation of Superficial Deltoid Ligament avulsion \\
\hline & & 2 & Superficial Deltoid Ligament 2-piece avulsion off tibia \\
\hline & & 3 & ATaFL 2-piece avulsion off fibula \\
\hline & & $4-7$ & Continued propagation of avulsions \\
\hline
\end{tabular}

Note. $\mathbf{X}$ indicates a syndesmotic injury. 
Table 10: Injury event sequences for initially Neutral tests, Runs 4-9 (BC:C).

\begin{tabular}{|c|c|c|c|}
\hline Run \# & Specimen \# & Event \# & Injury Description \\
\hline \multirow{4}{*}{4} & \multirow{4}{*}{$615 \mathrm{~L}$} & 1 & CFL avulsion off fibula \\
\hline & & 2 & $\begin{array}{l}\text { Either Superficial Deltoid Ligament avulsion off tibia or PTaFL } \\
\text { partial avulsion off fibula }\end{array}$ \\
\hline & & 3 & $\begin{array}{l}\text { Either Superficial Deltoid Ligament avulsion off tibia or PTaFL } \\
\text { partial avulsion off fibula }\end{array}$ \\
\hline & & 4 & Deep Deltoid Ligament avulsion off calcaneus \\
\hline \multirow{5}{*}{5} & \multirow{5}{*}{$794 \mathrm{~L}$} & 1 & Superficial Deltoid Ligament avulsion off tibia \\
\hline & & 2 & Deep Deltoid Ligament complete rupture \\
\hline & & 3 & $\mathbf{X}$ ATiFL complete rupture \\
\hline & & 4 & X $33 \mathrm{~mm}$ tear of Anterior IOM \\
\hline & & 5 & Artefactual Fibula Fracture \\
\hline \multirow{2}{*}{6} & \multirow{2}{*}{$801 \mathrm{~L}$} & 1 & Superficial Deltoid Ligament 2-piece avulsion off tibia \\
\hline & & 2 & Weber A Fibula Fracture \\
\hline \multirow{7}{*}{7} & \multirow{7}{*}{$612 \mathrm{~L}$} & 1 & $\mathbf{X}$ AiTiFL and ATiFL complete ruptures \\
\hline & & 2 & Antero-Lateral Joint Capsule and Posterior Capsule tears \\
\hline & & 3 & Superficial Deltoid Ligament sleeve avulsion off tibia \\
\hline & & 4 & $\mathbf{X}$ Distal IOM partial disruption \\
\hline & & 5 & X Weber C Fibula Fracture \\
\hline & & 6 & Talus component of Deep Deltoid Ligament complete rupture \\
\hline & & 7 & Artefactual Tibia Fracture \\
\hline \multirow{7}{*}{8} & \multirow{7}{*}{$752 \mathrm{~L}$} & 1 & Either Superficial Deltoid Ligament avulsion off tibia or off talus \\
\hline & & 2 & $\mathbf{X}$ AiTiFL avulsion off tibia \\
\hline & & 3 & Either Superficial Deltoid Ligament avulsion off tibia or off talus \\
\hline & & 4 & $\begin{array}{l}\text { Either partial rupture of calcaneus component or complete rupture of } \\
\text { talus component of Deep Deltoid Ligament }\end{array}$ \\
\hline & & 5 & $\begin{array}{l}\text { Either partial rupture of calcaneus component or complete rupture of } \\
\text { talus component of Deep Deltoid Ligament }\end{array}$ \\
\hline & & 6 & PTaFL complete rupture \\
\hline & & 7 & CFL complete rupture \\
\hline \multirow{6}{*}{9} & \multirow{6}{*}{$757 \mathrm{~L}$} & 1 & $\begin{array}{l}\text { Either Antero-Lateral Joint Capsule tear or Posterior Tibial Sheath } \\
\text { rupture }\end{array}$ \\
\hline & & 2 & Superficial Deltoid Ligament sleeve avulsion off tibia \\
\hline & & 3 & $\begin{array}{l}\text { Either calcaneus or talus component of Deep Deltoid Ligament } \\
\text { complete rupture }\end{array}$ \\
\hline & & 4 & $\begin{array}{l}\text { Either calcaneus or talus component of Deep Deltoid Ligament } \\
\text { complete rupture }\end{array}$ \\
\hline & & 5 & CFL complete rupture \\
\hline & & 6 & $\begin{array}{l}\text { Either Antero-lateral Joint Capsule tear or Posterior Tibial Sheath } \\
\text { rupture }\end{array}$ \\
\hline
\end{tabular}

Note. $\mathbf{X}$ indicates a syndesmotic injury. 
Table 11: Injury event sequences for initially Plantarflexed tests, Runs 10-13 (BC:D).

\begin{tabular}{|c|c|c|c|}
\hline Run \# & Specimen \# & Event \# & Injury Description \\
\hline \multirow{6}{*}{10} & \multirow{6}{*}{$680 \mathrm{R}$} & 1 & $\begin{array}{l}\text { CFL } 90 \% \text { rupture or Superficial Deltoid Ligament sleeve } \\
\text { avulsion off medial malleolus }\end{array}$ \\
\hline & & 2 & $\begin{array}{l}\text { CFL } 90 \% \text { rupture or Superficial Deltoid Ligament sleeve } \\
\text { avulsion off medial malleolus }\end{array}$ \\
\hline & & 3 & $\begin{array}{l}\text { Initiation of Deep Deltoid Ligament rupture (talus or } \\
\text { calcaneus component) }\end{array}$ \\
\hline & & 4 & $\begin{array}{l}\text { Deep Deltoid Ligament rupture (talus or calcaneus } \\
\text { component) }\end{array}$ \\
\hline & & 5 & $\begin{array}{l}\text { Deep Deltoid Ligament rupture (talus or calcaneus } \\
\text { component) }\end{array}$ \\
\hline & & 6 & PTaFL avulsion off fibula \\
\hline \multirow{4}{*}{11} & \multirow{4}{*}{$612 \mathrm{R}$} & 1 & CFL midsubstance rupture \\
\hline & & 2 & PTaFL partial rupture \\
\hline & & 3 & Tibia comminuted fracture \\
\hline & & 4 & Fibula comminuted fracture \\
\hline \multirow{4}{*}{12} & \multirow{4}{*}{$615 \mathrm{R}$} & 1 & $\begin{array}{l}\text { Superficial Deltoid Ligament sleeve avulsion off medial } \\
\text { malleolus }\end{array}$ \\
\hline & & 2 & CFL complete rupture (avulsed off fibula) \\
\hline & & 3 & PTaFL complete rupture (avulsed off fibula) \\
\hline & & $4-8$ & $\begin{array}{l}\text { Deep Deltoid Ligament avulsion of calcaneus component } \\
\text { and midsubstance rupture of talus component }\end{array}$ \\
\hline \multirow{3}{*}{13} & \multirow{3}{*}{$794 \mathrm{R}$} & 1 & $\begin{array}{l}\text { Superficial Deltoid Ligament sleeve avulsion off medial } \\
\text { malleolus }\end{array}$ \\
\hline & & 2 & $\begin{array}{l}\text { Deep Deltoid Ligament complete rupture (talus or calcaneus } \\
\text { component) }\end{array}$ \\
\hline & & 3 & $\begin{array}{l}\text { Deep Deltoid Ligament complete rupture (talus or calcaneus } \\
\text { component) }\end{array}$ \\
\hline
\end{tabular}

Note. An absence of syndesmotic injury for these specimens initially in plantarflexion (no black $\mathrm{Xs})$. 
Table 12: Injury event sequences for initially Dorsiflexed tests, Runs 14-17 (BC:E).

\begin{tabular}{|c|c|c|c|}
\hline Run \# & Specimen \# & Event \# & Injury Description \\
\hline \multirow{6}{*}{14} & \multirow{6}{*}{$682 \mathrm{R}$} & 1 & $\begin{array}{l}\text { Superficial Deltoid Ligament sleeve avulsion off medial } \\
\text { malleolus }\end{array}$ \\
\hline & & 2 & $\begin{array}{l}\mathbf{X} \text { ATiFL mild attenuation or initiation of Deep Deltoid } \\
\text { Ligament rupture (talus component) }\end{array}$ \\
\hline & & 3 & $\begin{array}{l}\mathbf{X} \text { ATiFL mild attenuation or further propagation of Deep } \\
\text { Deltoid rupture (talus component) }\end{array}$ \\
\hline & & 4 & CFL midsubstance rupture \\
\hline & & 5 & Deep Deltoid Ligament rupture (talus component) \\
\hline & & 6 & $\begin{array}{l}\text { Further propagation of Deltoid Ligament ruptures (talus } \\
\text { component) }\end{array}$ \\
\hline \multirow{5}{*}{15} & \multirow{5}{*}{$752 \mathrm{R}$} & 1 & Superficial Deltoid Ligament avulsion off medial malleolus \\
\hline & & 2 & $\mathbf{X}$ ATiFL avulsion off anterior-lateral distal tibia \\
\hline & & 3 & $\begin{array}{l}\text { Initiation of IOM tear or Deep Deltoid Ligament rupture } \\
\text { (talus or calcaneus component) }\end{array}$ \\
\hline & & 4 & $\mathbf{X}$ Anterior IOM tear \\
\hline & & 5 & $\begin{array}{l}\text { Deep Deltoid Ligament rupture propagation (talus or } \\
\text { calcaneus component) }\end{array}$ \\
\hline \multirow{4}{*}{16} & \multirow{4}{*}{$757 \mathrm{R}$} & 1 & X ATiFL complete rupture \\
\hline & & 2 & Fibula Fracture \\
\hline & & 3 & ATaFL mild attenuation \\
\hline & & 4 & $\mathbf{X}$ Anterior IOM tear \\
\hline \multirow{5}{*}{17} & \multirow{5}{*}{$801 \mathrm{R}$} & 1 & $\begin{array}{l}\text { Superficial Deltoid Ligament sleeve avulsion off medial } \\
\text { malleolus }\end{array}$ \\
\hline & & 2 & $\begin{array}{l}\text { Deep Deltoid Ligament rupture initiation (talus or calcaneus } \\
\text { component) }\end{array}$ \\
\hline & & 3 & $\mathbf{X}$ ATiFL complete rupture \\
\hline & & 4 & $\begin{array}{l}\text { Deep Deltoid Ligament rupture propagation (talus or } \\
\text { calcaneus component) }\end{array}$ \\
\hline & & 5 & $\mathbf{X}$ Weber B Fibula Fracture \\
\hline
\end{tabular}

Note. A consistency of syndesmotic injury for all specimens initially in dorsiflexion. $\mathbf{X}$ indicates a syndesmotic injury.

As an example (Mait et al., 2017a), the injuries documented for Run 15 (Specimen 752R) in an initially dorsiflexed posture are reported (Figure 21). Two deltoid ligament and syndesmotic ligament injuries were documented in this specimen. The superficial deltoid complex avulsed off the medial malleolus and both components of the deep deltoid complex sustained complete midsubstance ruptures. Also, the ATiFL avulsed off the antero-lateral distal tibia (approx. 2 x $1 \mathrm{x}$ $1 \mathrm{~cm}$ bony fragment) with a $4 \mathrm{~cm}$ (approximate) tear of the anterior IOM propagating proximally from the tibiofibular syndesmosis joint. 


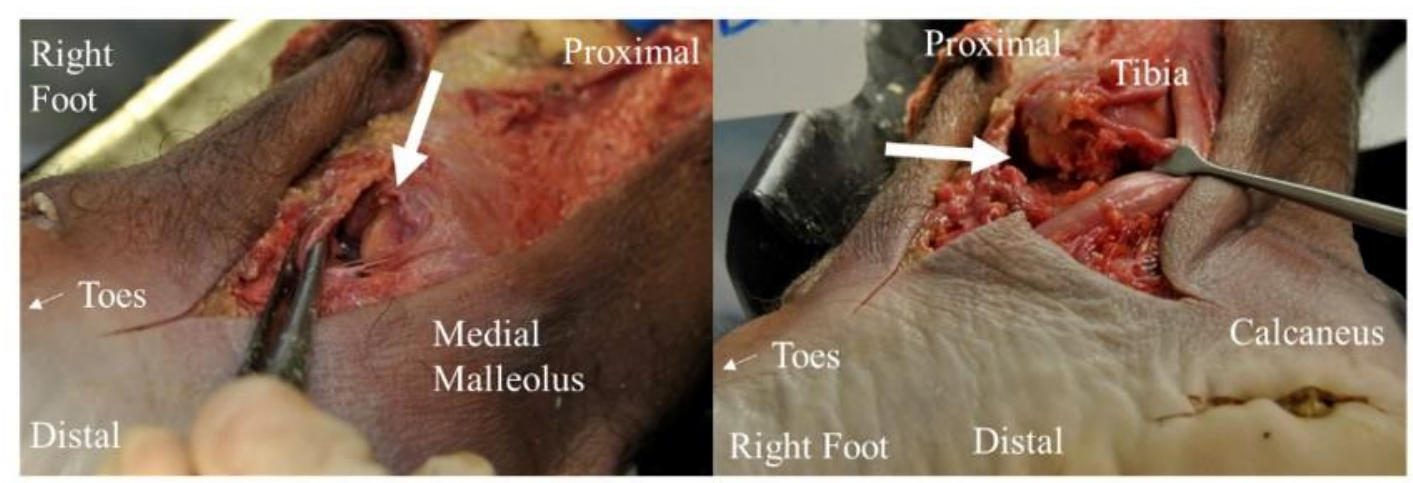

Superficial Deltoid: Sleeve Avulsion

Deep Deltoid:

Complete Rupture of both Components

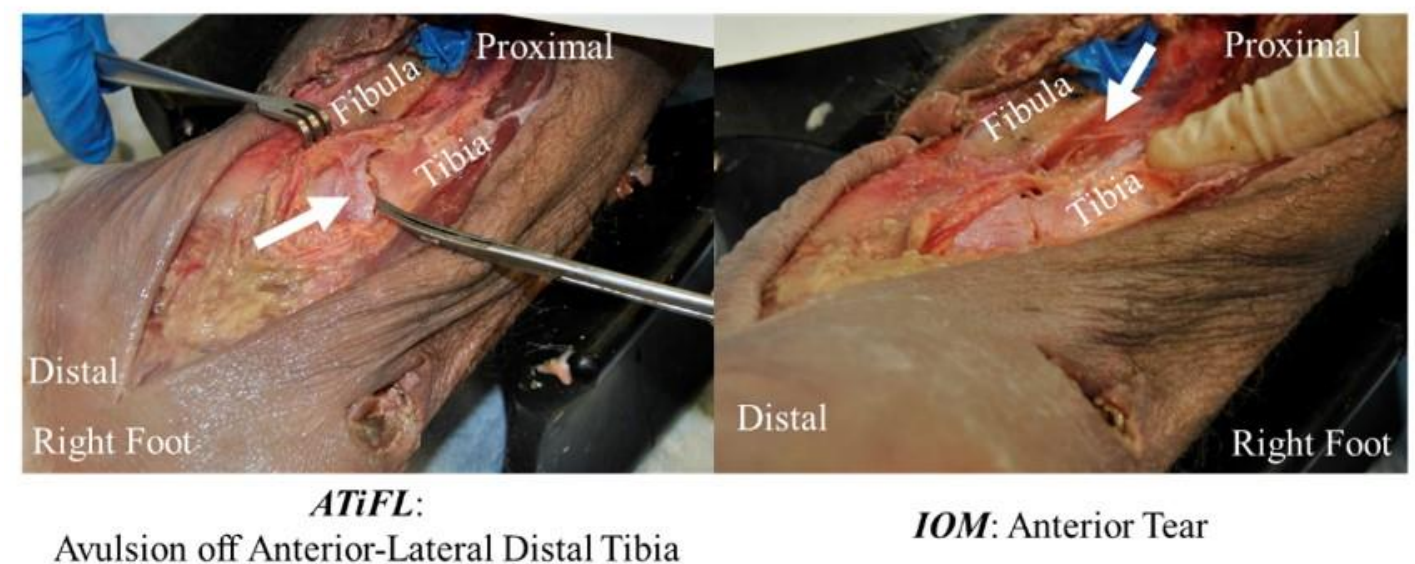

Figure 21: Dissection photographs documenting diagnosed injuries in the ankle (Example: Run 15, Specimen 752R, Dorsiflexion).

The injury timing determination process is illustrated below using the data correlated to the necropsy findings for this specimen. Post-dissection, mechanical response data traces (kinetic, audio, and kinematic) are plotted against time to distinguish possible injury events throughout the rotation cycle (Mait et al., 2017a). Kinetic data for tibia and fibula strain and moment (Mz from the tibia load cell) are plotted against time with input rotation (Instron Rotation) included for comparison. Separate plots were generated for moment, external microphone, and bone-mounted microphone (acoustic emission) data traces plotted against time, again with input rotation included for comparison (Figure 22). Also, kinematic data for the calcaneus, fibula, talus, and navicular were plotted against time relative to the tibia coordinate system for linear and angular displacements (rotations and translations) throughout the rotation cycle (Figure 23). Injury events 
during the rotation cycle were distinguished by sudden changes in the data traces and are highlighted in boxes.
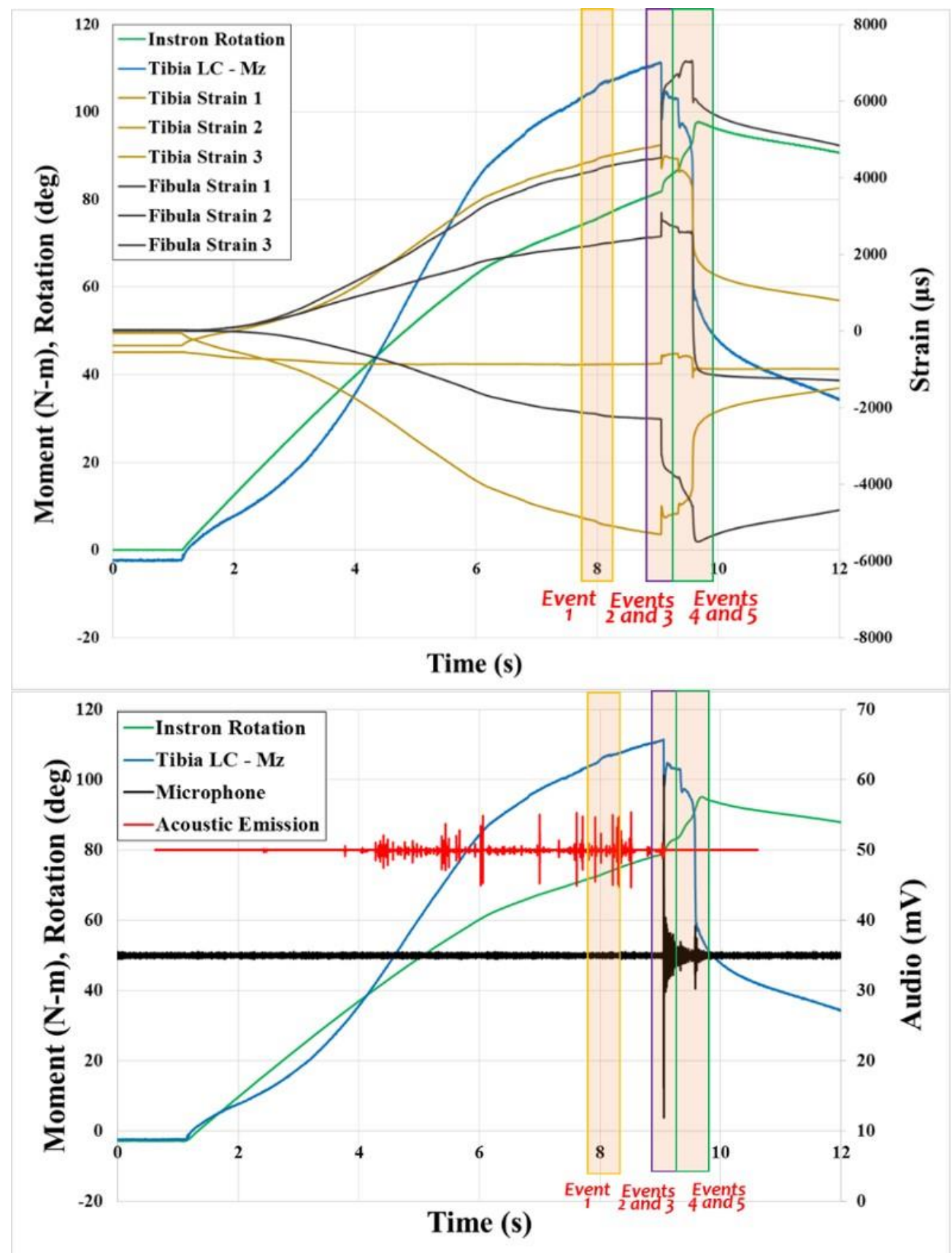

Figure 22: Injury event timing example (Run 15, Specimen 752R, Dorsiflexion) plots for (top) moment and strain and (bottom) moment and acoustic data traces. Injury events are highlighted: Event 1 in a yellow box, Events 2 and 3 in a purple box, and Events 4 and 5 in a green box. Note: Tibia LC - Mz refers to the reaction moment measured by the load cell fixed to the tibia plateau, Instron Rotation refers to the input rotation cycle, Microphone refers to the external microphone, and Acoustic Emission refers to the bone-mounted (on fibula) microphone acoustic sensor. 
As an example of the injury sequence determination review process (Table 13) for Run 15 on Specimen 752R (Mait et al., 2017a), the five events distinguished in Figure 22 and Figure 23 were matched to diagnosed injuries (Figure 21). From the large spike in external microphone (Figure 22) in conjunction with abrupt changes in tibia and fibula strain and moment, the ATiFL avulsion off the tibia was attributed to event 2 . Next, from the small change in moment but sharp changes in talus translations only (Figure 22 and Figure 23), combined with the spikes in acoustic emission, the avulsion of the smaller, less stout superficial deltoid complex was attributed to event 1. Events 4 and 5 both had large, abrupt drops in moment (Figure 22). Event 5 had the larger drop in moment and significant perturbations in the talus and calcaneus kinematics (Figure 23). Event 4 had a smaller drop in moment and perturbations in all bone motions, but most noticeably in the fibula, therefore was attributed to the IOM tear. Lastly, this left the deep deltoid complex (larger, stouter structure than the IOM) complete rupture attributed to event 5, confirmed with the large burst in the external microphone data trace (Figure 22). Matching injuries to events 3, 4, and 5 (Table 13) illustrate the uncertainties the expert panel experienced during the injury timing determination process. Relative to time, events 3, 4, and 5 occurred close to each other (Figure 22 and Figure 23). While events 1 and 2 were distinguished with certainty, initiation and subsequent rupture of the IOM and deep deltoid complex (events 3-5) were assigned confidence levels of unknown and probable. 


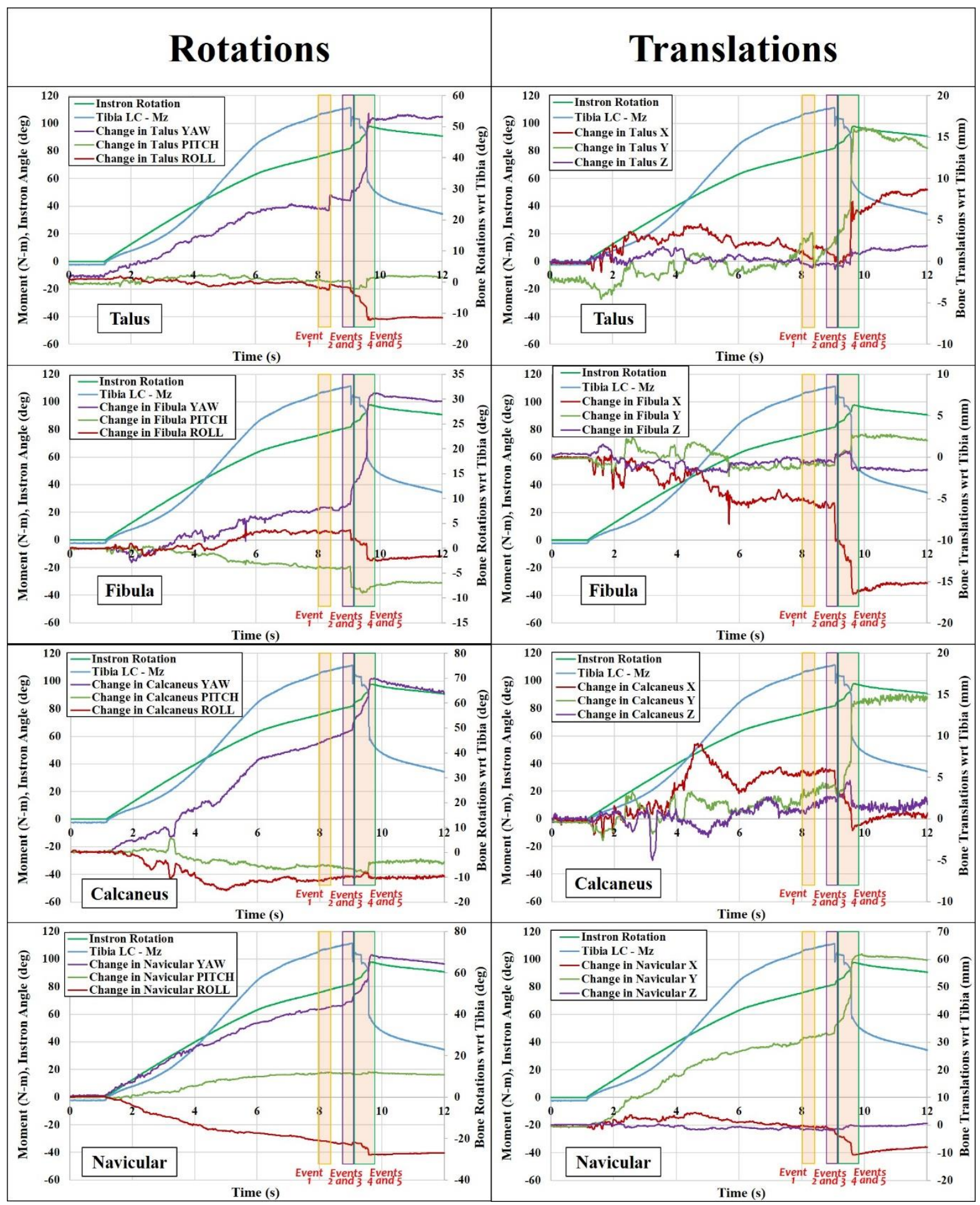

Figure 23: Injury event timing example (Run 15, Specimen 752R, Dorsiflexion) plots for kinematic data traces of bone-specific (left) rotations and (right) translations. All data indicates the change in orientation or position of each bone in the tibia coordinate system. Injury events are highlighted: Event 1 in a yellow box, Events 2 and 3 in a purple box, and Events 4 and 5 in a green box. Note: Tibia LC - Mz refers to the reaction moment measured by the load cell fixed to the tibia plateau and Instron Rotation refers to the input rotation cycle. 
Table 13: Example (Run 15, Specimen 752R, Dorsiflexion) injury event sequence determination using all diagnostic tools (kinetics, kinematics, strain, and audio). Corresponding injuries are indicated for each injury event.

\begin{tabular}{|c|c|c|c|c|c|c|c|c|c|}
\hline $\begin{array}{c}\text { Event } \\
\#\end{array}$ & $\begin{array}{l}\text { Start } \\
\text { Time } \\
\text { (s) }\end{array}$ & $\begin{array}{c}\text { Instron } \\
\text { Angle } \\
\left(^{\circ}\right)\end{array}$ & $\begin{array}{l}\text { Ext. } \\
\text { Rot. } \\
\left({ }^{\circ}\right)^{*}\end{array}$ & $\begin{array}{c}\text { Effect } \\
\text { on } \\
\text { Moment }\end{array}$ & $\begin{array}{c}\text { Effect on } \\
\text { Kinematics }\end{array}$ & $\begin{array}{l}\text { Effect } \\
\text { on } \\
\text { Strain }\end{array}$ & $\begin{array}{l}\text { Effect on } \\
\text { Audio } \uparrow\end{array}$ & $\begin{array}{l}\text { Corresponding } \\
\text { Injury }\end{array}$ & Confidence \\
\hline 1 & 8.00 & 75.7 & 44.1 & $\begin{array}{l}\text { Small } \\
\text { change in } \\
\text { slope } \\
(\sim 1 \mathrm{~N}-\mathrm{m})\end{array}$ & $\begin{array}{l}\text { Sharp changes in all } \\
\text { Talus translations } \\
\text { and rotations post- } \\
\text { event }\end{array}$ & $\begin{array}{l}\text { Small } \\
\text { changes in } \\
\text { fibula and } \\
\text { tibia strain }\end{array}$ & $\begin{array}{l}\text { Series of } \\
\text { spikes in } \\
\mathrm{AE}\end{array}$ & $\begin{array}{l}\text { Superficial Deltoid } \\
\text { sleeve avulsion }\end{array}$ & Certain \\
\hline 2 & 9.01 & 81.6 & 49.0 & $\begin{array}{l}\text { Abrupt } \\
\text { drop } \\
(\sim 12 \mathrm{~N}- \\
\mathrm{m})\end{array}$ & $\begin{array}{l}\text { Sharp changes in } \\
\text { most bone rotations } \\
\text { and translations, } \\
\text { especially for Fibula } \\
\text { and Talus }\end{array}$ & $\begin{array}{l}\text { Abrupt } \\
\text { changes in } \\
\text { fibula and } \\
\text { tibia strain }\end{array}$ & $\begin{array}{l}\text { Large spike } \\
\text { in Mic and } \\
\text { small spike } \\
\text { in AE }\end{array}$ & $\begin{array}{l}\text { ATiFL rupture/ } \\
\text { Tibia avulsion }\end{array}$ & Certain \\
\hline 3 & 9.17 & 85.1 & 54.4 & $\begin{array}{l}\text { Small, } \\
\text { gradual } \\
\text { drop } \\
(\sim 1.5 \mathrm{~N}- \\
\mathrm{m})\end{array}$ & $\begin{array}{l}\text { Changes in Calc. and } \\
\text { Talus yaw and roll } \\
\text { with respect to Tibia }\end{array}$ & $\begin{array}{l}\text { Small } \\
\text { changes in } \\
\text { tibia strain }\end{array}$ & $\begin{array}{l}\text { Spike in } \\
\text { Mic within } \\
\text { cone after } \\
\text { Tibia } \\
\text { Avulsion }\end{array}$ & $\begin{array}{l}\text { Initiation of Deep } \\
\text { Deltoid rupture or } \\
\text { IOM tear? }\end{array}$ & Possible \\
\hline 4 & 9.34 & 86.9 & 56.4 & $\begin{array}{l}\text { Abrupt } \\
\text { drop } \\
(\sim 6 \mathrm{~N}-\mathrm{m})\end{array}$ & $\begin{array}{l}\text { Abrupt changes in } \\
\text { Talus yaw and roll } \\
\text { with respect to Tibia; } \\
\text { Abrupt changes in all } \\
\text { Calc. and Nav. } \\
\text { Rotations; Abrupt } \\
\text { changes in most } \\
\text { Talus and Calc. } \\
\text { translations; } \\
\text { Perturbations in most } \\
\text { Fibula translations } \\
\text { and rotations }\end{array}$ & $\begin{array}{l}\text { Small, } \\
\text { abrupt } \\
\text { changes in } \\
\text { fibula and } \\
\text { tibia strain }\end{array}$ & $\begin{array}{l}\text { Spike in } \\
\text { Mic }\end{array}$ & IOM tear & Probable \\
\hline 5 & 9.43 & 90.1 & 59.6 & $\begin{array}{l}\text { Abrupt } \\
\text { drop } \\
(\sim 40 \mathrm{~N}- \\
\mathrm{m})\end{array}$ & $\begin{array}{l}\text { Sharp changes in } \\
\text { most bone rotations } \\
\text { and translations; } \\
\text { most noticeable in } \\
\text { Talus, Fibula, and } \\
\text { Calc. }\end{array}$ & $\begin{array}{l}\text { Abrupt } \\
\text { changes in } \\
\text { fibula and } \\
\text { tibia strain }\end{array}$ & $\begin{array}{l}\text { Spike in } \\
\text { Mic }\end{array}$ & $\begin{array}{l}\text { Deep Deltoid } \\
\text { rupture } \\
\text { propagation }\end{array}$ & Probable \\
\hline
\end{tabular}

*This is defined as the change in calcaneus rotation about the tibia $\mathrm{z}$-axis in the transverse plane from time $=0$ seconds until the time of the event.

†Mic refers to external microphone and $\mathrm{AE}$ refers to bone-mounted (on fibula) microphone acoustic sensor.

From the injury sequences identified in Table 9, Table 10, Table 11, and Table 12, injury incidences by anatomical structure and location within the ankle were determined for all tests (Table 14). Syndesmotic injury was observed in 5 of the 9 initially neutral specimens, 0 of 4 for initially plantarflexed, and 4 of 4 for initially dorsiflexed (Table 14). Medial ankle injury to the deltoid ligament complex was also common. The superficial deltoid complex was injured in 9 of 9 neutral specimens and 3 of 4 specimens for both plantarflexion and dorsiflexion. These injuries propagated into the deep deltoid for 5 of 9 neutral specimens, 3 of 4 specimens for plantarflexion, 
and 3 of 4 specimens for dorsiflexion. Lateral ankle injury was observed in 6 of 9 neutral specimens, 1 of 4 dorsiflexion specimens, and 3 of 4 plantarflexion specimens. These results suggest that foot flexion posture possibly influences injury patterns in the ankle caused by applied external foot rotation.

Table 14: Injury incidence for clinically significant documented injuries in the initially neutral (n $=9)$, plantarflexed $(n=4)$, and dorsiflexed $(n=4)$ ankle postures.

\begin{tabular}{|c|c|c|c|}
\hline Anatomical Structure & Plantarflexion & Neutral & Dorsiflexion \\
\hline ATiFL & 0 & 5 & 4 \\
\hline IOM & 0 & 3 & 2 \\
\hline PTiFL & 0 & 0 & 0 \\
\hline $\begin{array}{l}\text { Fibula Fracture } \\
\text { (Weber B or C) }\end{array}$ & 0 & 1 & 1 \\
\hline Superficial Deltoid & 3 & 9 & 3 \\
\hline Deep Deltoid & 3 & 5 & 3 \\
\hline ATaFL & 0 & 2 & 1 \\
\hline CFL & 3 & 3 & 1 \\
\hline PTaFL & 3 & 2 & 0 \\
\hline $\begin{array}{c}\text { Fibula Fracture } \\
\text { (Weber A) }\end{array}$ & 0 & 1 & 0 \\
\hline $\begin{array}{l}\text { Tibia Fracture } \\
\text { (Artefactual) }\end{array}$ & 1 & 1 & 0 \\
\hline $\begin{array}{l}\text { Fibula Fracture } \\
\text { (Artefactual) }\end{array}$ & 1 & 1 & 1 \\
\hline
\end{tabular}

Note. Clinically significant ligament and bone fractures are noted beginning with syndesmotic region, then deltoid, and then lateral. Ligament injury severity varied among specimens: all severities (attenuations, avulsions, partial tears, and midsubstance ruptures) are grouped together. Artefactual (either through kinematic marker mounts or potting fixtures) tibia and fibula fractures are distinguished separately.

For a qualitative comparison of injury sequence across boundary conditions (initially neutral: $\mathrm{BC}: \mathrm{B}$ and $\mathrm{BC}: \mathrm{C}$, initially plantarflexed: $\mathrm{BC}: \mathrm{D}$, and initially dorsiflexed: $\mathrm{BC}: \mathrm{E})$, injury results were illustrated on one specimen from each of the four test conditions (Figure 24). Approximate locations of pertinent ankle ligaments are illustrated on the tibia, fibula, talus, navicular, and calcaneus bones within the ankle. Injury sequences are shown on these illustrations and in boxes next to each illustration for reference. For the initially neutral posture, the specimen under $\mathrm{BC}: \mathrm{B}$ (Figure 24, top) sustained injuries to the syndesmosis, medial, and lateral ankle regions, however 
the specimen under BC:C (Figure 24, middle-top) sustained injuries to the medial and lateral ankle regions, which presumably offloaded the syndesmosis. For the initially dorsiflexed posture (Figure 24, middle-bottom), the specimen sustained injuries to the syndesmosis and medial ankle regions, however the initially plantarflexed specimen (Figure 24, bottom) sustained injuries to the medial and lateral ankle regions, again presumably offloading the syndesmosis. 


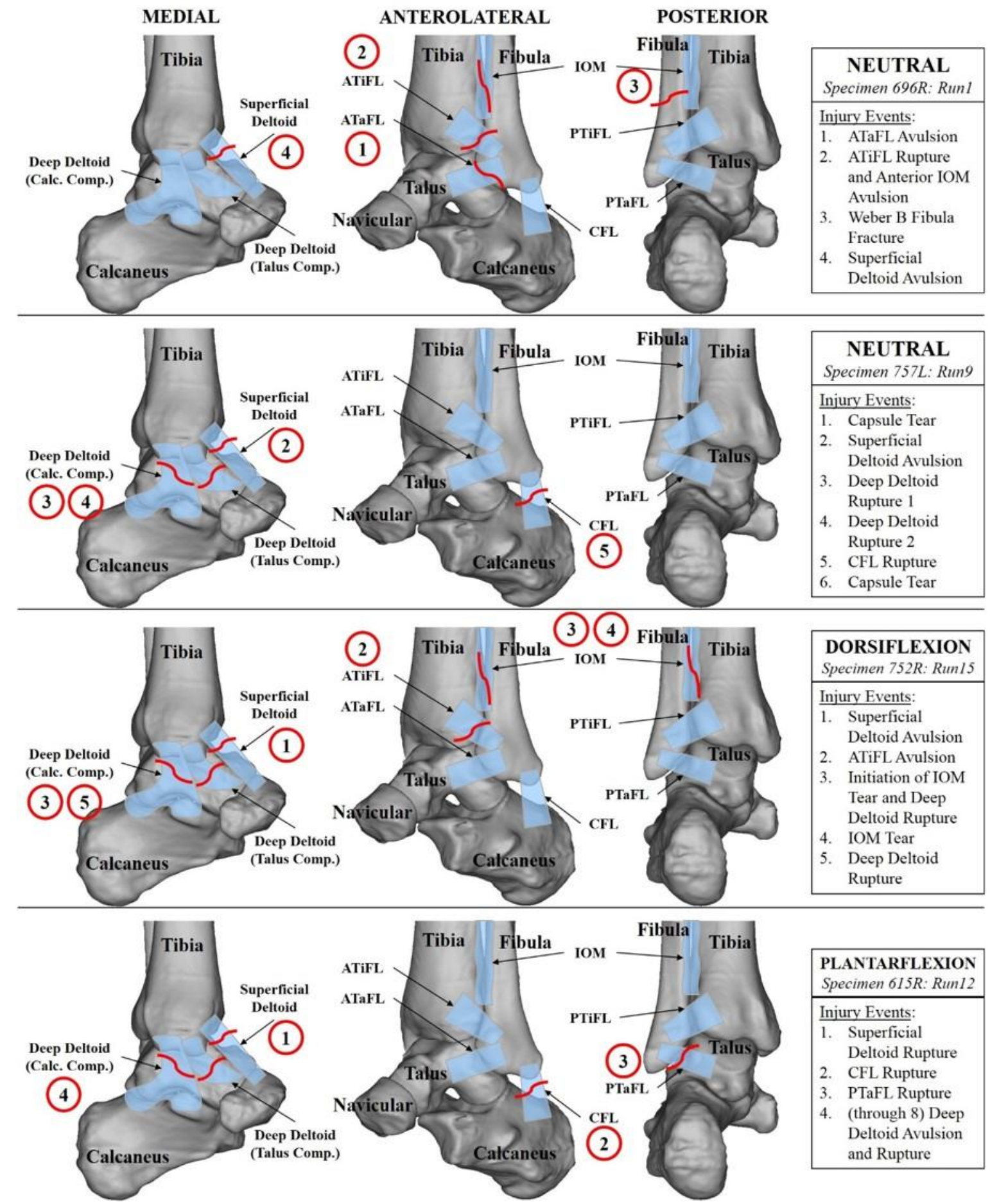

Figure 24: Representative images of injury event sequences for (top) neutral test with calcaneus in fixed orientation (Run 1, Specimen 696R), (top-middle) neutral test with calcaneus free to invert/evert (Run 9, Specimen 757L), (bottom-middle) initially dorsiflexed test (Run 15, Specimen 752R), and (bottom) initially plantarflexed test (Run

12, Specimen 615R). Medial, Antero-Lateral, and Posterior views are shown with pertinent ankle ligaments and bones labelled. Boxes on the right detail the injury events, with circles in the illustrations highlighting these events. 
With all the efforts to determine injury incidences (Table 14) and injury sequences (Table 9, Table 10, Table 11, Table 12, and Figure 24), the injury timing, relative to external foot rotation (ER), of each event during the rotation cycle was ascertained. Injury events and corresponding ER values are listed in Table 15, Table 16, Table 17, and Table 18 for the initially neutral (Runs 1-3, BC:B and then Runs 4-9, BC:C), plantarflexed (Runs 10-13, BC:D), and dorsiflexed (Runs 14-17, BC:E) ankle flexion postures. These ER values were used to determine an injury occurrence limit for syndesmotic injury across all initial ankle flexion postures (see Chapter 4). In Runs 12 and 13 (plantarflexion), calcaneus kinematic data was partially lost during testing. Therefore, the ER values were estimated based on talus axial rotation about the tibia long-axis. Calcaneus axial rotation was plotted relative to talus axial rotation ( $\Delta$ yaw, about the tibia long-axis) for all test runs, regardless of initial ankle flexion posture. A linear relationship was found between these two rotations with high determination $\left(\mathrm{R}^{2}>0.94\right.$ across all tests), therefore talus axial rotation was determined as a good estimator of calcaneus axial rotation. To keep testing initial conditions similar, the slopes of the calcaneus vs. talus axial rotation plots from Runs 10 and 11 were used to scale the talus axial rotations in Runs 12 and 13. Thus, these slope values from Runs 10 and 11 give ranges of the minimum to maximum calcaneus axial rotation (ER) estimations for Runs 12 and 13 (Table 17).

Table 15: Injury timings relative to external foot rotation (ER) measured during tests (Runs 1-3, BC:B) in the initially neutral ankle flexion posture.

\begin{tabular}{cclcc}
\hline \multirow{2}{*}{ Run \# } & Specimen \# & \multicolumn{1}{c}{ Injury Event } & Ankle Region & External Rotation $\left(^{\circ}\right)$ \\
\hline \multirow{3}{*}{1} & \multirow{3}{*}{$696 \mathrm{R}$} & 1: ATaFL Avulsion & Lateral & 28.3 \\
& & 2: ATiFL Rupture/IOM Avulsion & Syndesmosis & 33.5 \\
& & 3: Weber B Fibula Fracture & Syndesmosis & 40.3 \\
& & 4: Superficial Deltoid Avulsion & Deltoid & 48.0 \\
\hline \multirow{2}{*}{2} & \multirow{2}{*}{$680 \mathrm{~L}$} & 1: Superficial Deltoid Avulsion & Deltoid & 37.2 \\
& & 2: AiTiFL Rupture & Syndesmosis & 45.3 \\
\hline \multirow{3}{*}{3} & \multirow{3}{*}{$682 \mathrm{~L}$} & 1: Superficial Deltoid Initiation & Deltoid & 35.2 \\
& & 2: Superficial Deltoid Avulsion & Deltoid & 40.5 \\
& & 3: ATaFL Avulsion & Lateral & 44.3 \\
& & 4 to 7: Avulsion Propagations & Lateral/Deltoid & 49.6 to 55.1 \\
\hline
\end{tabular}


Table 16: Injury timings relative to external foot rotation (ER) measured during tests (Runs 4-9, BC:C) in the initially neutral ankle flexion posture.

\begin{tabular}{|c|c|c|c|c|}
\hline Run \# & Specimen \# & Injury Event & Ankle Region & External Rotation $\left(^{\circ}\right)$ \\
\hline \multirow{3}{*}{4} & \multirow{3}{*}{$615 \mathrm{~L}$} & 1: Either CFL or PTaFL Avulsion & Lateral & 35.6 \\
\hline & & $\begin{array}{l}\text { 2: Either CFL, PTaFL, or Superficial Deltoid } \\
\text { Avulsion }\end{array}$ & Lateral/Deltoid & 43.2 \\
\hline & & 3: Deep and Superficial Deltoid Avulsion & Deltoid & 54.3 \\
\hline \multirow{5}{*}{5} & \multirow{5}{*}{$794 \mathrm{~L}$} & 1: Superficial Deltoid Avulsion & Deltoid & 20.1 \\
\hline & & 2: Deep Deltoid Rupture & Deltoid & 24.3 \\
\hline & & 3: ATiFL Rupture & Syndesmosis & 30.3 \\
\hline & & 4: IOM Tear & Syndesmosis & 41.2 \\
\hline & & 5: Fibula Fracture & Artefactual* & 52.0 \\
\hline \multirow{2}{*}{6} & \multirow{2}{*}{$801 \mathrm{~L}$} & 1: Superficial Deltoid Avulsion & Deltoid & 31.1 \\
\hline & & 2: Weber A Fibula Fracture & Lateral & 53.2 \\
\hline \multirow{6}{*}{7} & \multirow{6}{*}{$612 \mathrm{~L}$} & 1: ATiFL and AiTiFL Rupture & Syndesmosis & 28.6 \\
\hline & & 2: Superficial Deltoid Avulsion & Deltoid & 36.4 \\
\hline & & 3: IOM/IOL Disruption & Syndesmosis & 46.9 \\
\hline & & 4: Weber C Fibula Fracture & Syndesmosis & 51.9 \\
\hline & & 5: Deep Deltoid Rupture & Deltoid & 55.7 \\
\hline & & 6: Tibia Fracture & Artefactual* & 60.9 \\
\hline \multirow{7}{*}{8} & \multirow{7}{*}{$752 \mathrm{~L}$} & 1: Superficial Deltoid Avulsion 1 & Deltoid & 26.0 \\
\hline & & 2: AiTiFL Avulsion/ATiFL Attenuation & Syndesmosis & 31.7 \\
\hline & & 3: Superficial Deltoid Avulsion 2 & Deltoid & 36.4 \\
\hline & & 4: Deep Deltoid Rupture 1 & Deltoid & 40.6 \\
\hline & & 5: Deep Deltoid Rupture 2 & Deltoid & 47.3 \\
\hline & & 6: PTaFL Rupture & Lateral & 60.4 \\
\hline & & 7: CFL Rupture & Lateral & 62.3 \\
\hline \multirow{4}{*}{9} & \multirow{4}{*}{$757 \mathrm{~L}$} & 1: Superficial Deltoid Avulsion & Deltoid & 33.4 \\
\hline & & 2: Deep Deltoid Rupture 1 & Deltoid & 39.7 \\
\hline & & 3: Deep Deltoid Rupture 2 & Deltoid & 51.4 \\
\hline & & 4: CFL Rupture & Lateral & 59.8 \\
\hline
\end{tabular}


Table 17: Injury timings relative to external foot rotation (ER) measured during tests (Runs 10-13, $\mathrm{BC}: \mathrm{D})$ in the initially plantarflexed ankle flexion posture.

\begin{tabular}{|c|c|c|c|c|}
\hline Run \# & Specimen \# & Injury Event & Ankle Region & External Rotation $\left(^{\circ}\right)$ \\
\hline \multirow{6}{*}{10} & \multirow{6}{*}{$680 \mathrm{R}$} & $\begin{array}{l}\text { 1: CFL Rupture or Superficial } \\
\text { Deltoid Avulsion }\end{array}$ & Lateral/Deltoid & 21.6 \\
\hline & & $\begin{array}{l}\text { 2: CFL Rupture or Superficial } \\
\text { Deltoid Avulsion }\end{array}$ & Lateral/Deltoid & 22.9 \\
\hline & & $\begin{array}{l}\text { 3: Initiation of Deep Deltoid } \\
\text { Rupture }\end{array}$ & Deltoid & 32.9 \\
\hline & & $\begin{array}{l}\text { 4: Propagation of Deep Deltoid } \\
\text { Rupture } 1\end{array}$ & Deltoid & 43.1 \\
\hline & & $\begin{array}{l}\text { 5: Propagation of Deep Deltoid } \\
\text { Rupture } 2\end{array}$ & Deltoid & 45.0 \\
\hline & & 6: PTaFL Avulsion & Lateral & 57.3 \\
\hline \multirow{4}{*}{11} & \multirow{4}{*}{$612 \mathrm{R}$} & 1: CFL Rupture & Lateral & 20.5 \\
\hline & & 2: PTaFL Partial Rupture & Lateral & 28.6 \\
\hline & & 3: Tibia Fracture & Artefactual* & 29.8 \\
\hline & & 4: Fibula Fracture & Artefactual* & 39.6 \\
\hline \multirow{5}{*}{12} & \multirow{5}{*}{$615 \mathrm{R}$} & 1: Superficial Deltoid Rupture & Deltoid & 17.1 to $20.9^{\wedge}$ \\
\hline & & 2: CFL Rupture & Lateral & 20.6 to $25.1^{\wedge}$ \\
\hline & & 3: PTaFL Avulsion & Lateral & 22.8 to $27.7^{\wedge}$ \\
\hline & & $\begin{array}{l}\text { 4: Initiation of Deep Deltoid } \\
\text { Rupture }\end{array}$ & Deltoid & 23.8 to $29.0^{\wedge}$ \\
\hline & & $\begin{array}{l}5 \text { to 8: Deep Deltoid Rupture } \\
\text { Propagations }\end{array}$ & Deltoid & 25.3 to $50.7^{\wedge}$ \\
\hline \multirow{3}{*}{13} & \multirow{3}{*}{ 794R } & 1: Superficial Deltoid Avulsion & Deltoid & 16.0 to $19.5^{\wedge}$ \\
\hline & & $\begin{array}{l}\text { 2: Deep Deltoid Rupture: Talus } \\
\text { Component }\end{array}$ & Deltoid & 17.9 to $21.8^{\wedge}$ \\
\hline & & $\begin{array}{l}\text { 3: Deep Deltoid Rupture: } \\
\text { Calcaneus Component }\end{array}$ & Deltoid & 24.6 to $29.9^{\wedge}$ \\
\hline
\end{tabular}

*Artefactual fractures occurred at the insertion points of the screws attaching either the potting cup or kinematic marker to the bones

${ }^{\wedge}$ Calcaneus bone motion was lost during parts of Runs 12 and 13, therefore external rotation values are given as a range of minimum to maximum estimates using talus axial rotation values (scaled by slope of the linear regression found for talus vs. calcaneus axial rotations in Runs 10 and 11) about the tibia long-axis 
Table 18: Injury timings relative to external foot rotation (ER) measured during tests (Runs 14-17, $\mathrm{BC}: \mathrm{E})$ in the initially dorsiflexed ankle flexion posture.

\begin{tabular}{|c|c|c|c|c|}
\hline Run \# & Specimen \# & Injury Event & Ankle Region & External Rotation $\left(^{\circ}\right)$ \\
\hline \multirow{6}{*}{14} & \multirow{6}{*}{$682 \mathrm{R}$} & $\begin{array}{l}\text { 1: Superficial Deltoid Sleeve } \\
\text { Avulsion }\end{array}$ & Deltoid & 38.7 \\
\hline & & $\begin{array}{l}\text { 2: Initiation of Deep Deltoid } \\
\text { Rupture or ATiFL Attenuation }\end{array}$ & Deltoid/Syndesmosis & 45.5 \\
\hline & & $\begin{array}{l}\text { 3: Propagation of Deep Deltoid } \\
\text { Rupture or ATiFL Attenuation }\end{array}$ & Deltoid/Syndesmosis & 49.6 \\
\hline & & 4: CFL Rupture & Lateral & 52.6 \\
\hline & & $\begin{array}{l}\text { 5: Propagation of Deep Deltoid } \\
\text { Rupture } 1\end{array}$ & Deltoid & 60.3 \\
\hline & & $\begin{array}{l}\text { 6: Propagation of Deep Deltoid } \\
\text { Rupture } 2\end{array}$ & Deltoid & 66.5 \\
\hline \multirow{5}{*}{15} & \multirow{5}{*}{$752 \mathrm{R}$} & 1: Superficial Deltoid Avulsion & Deltoid & 44.1 \\
\hline & & 2: Tibia Avulsion/ATiFL Rupture & Syndesmosis & 49.0 \\
\hline & & $\begin{array}{l}\text { 3: Initiation of Deep Deltoid } \\
\text { Rupture or IOM Tear }\end{array}$ & Deltoid/Syndesmosis & 54.4 \\
\hline & & 4: IOM Tear & Syndesmosis & 56.4 \\
\hline & & $\begin{array}{l}\text { 5: Propagation of Deep Deltoid } \\
\text { Rupture }\end{array}$ & Deltoid & 59.6 \\
\hline \multirow{4}{*}{16} & \multirow{4}{*}{$757 \mathrm{R}$} & 1: ATiFL Rupture & Syndesmosis & 39.0 \\
\hline & & 2: Fibula Fracture & Artefactual* & 46.0 \\
\hline & & 3: ATaFL Attenuation & Lateral & 62.4 \\
\hline & & 4: IOM Tear & Syndesmosis & 71.1 \\
\hline \multirow{5}{*}{17} & \multirow{5}{*}{$801 \mathrm{R}$} & 1: Superficial Deltoid Avulsion? & Deltoid & 26.2 \\
\hline & & $\begin{array}{l}\text { 2: Initiation of Deep Deltoid or } \\
\text { Superficial Deltoid Avulsion }\end{array}$ & Deltoid & 31.5 \\
\hline & & 3: ATiFL Rupture & Syndesmosis & 38.7 \\
\hline & & 4: Deep Deltoid Rupture & Deltoid & 48.5 \\
\hline & & 5: Weber B Fibula Fracture & Syndesmosis & 58.0 \\
\hline
\end{tabular}

*Artefactual fractures occurred at the insertion points of the screws attaching either the potting cup or kinematic marker to the bones

\subsection{Discussion and Conclusions}

This chapter examined the functionally relevant boundary conditions $(\mathrm{BC}: \mathrm{B})$, implemented in Chapter 2 for non-injurious tests (BC:A), in new experiments investigating syndesmotic injury during excessive external foot rotation (Objective 2). Further iterations of boundary conditions improvements were also implemented in this chapter to find a set of external boundaries imposed 
on the foot that would be realistic for syndesmotic injury re-creation, yet mechanically tractable for proper ankle mechanical response data collection. From the excessive rotation experiments, the sequence of injury in ankle ligaments for legs, subject to the proposed injury mechanism (external foot rotation), in an initially neutral ankle flexion posture (Objective 2) was determined. Sensitivity of this sequence to other initial ankle flexion postures (dorsiflexion and plantarflexion; Objective 3) was also determined. Finally, this chapter tested non-senescent, sizable cadavers (Table 6 ) in order to re-create ligamentous syndesmotic injury instead of fibula fracture at failure as in previous studies (Markolf et al., 1989; Michelson et al., 1997).

Medial ligament injury (superficial and deep deltoid complexes) has been proposed to be directly correlated to forceful external foot rotation (Boytim et al., 1991; Guise, 1976; Teramoto et al., 2008; Williams et al., 2007). The results of this chapter upheld this proposal with 15 of 17 specimens sustaining a medial ligament injury, regardless of initial ankle flexion (Table 9, Table 10, Table 11, Table 12, and Table 14). Wei et al. (2012b) reported a similarly high incidence of injury to medial ligaments: 5 of 6 specimens suffered deltoid injuries, and further reported an absence of injury to the PTiFL, which is also consistent with the results in this chapter (Table 14). However, Wei et al. (2012b) reported only one specimen with injury to the ATiFL and no injuries to the IOM, whereas this chapter reported that 9 specimens suffered an ATiFL injury, with 5 of those 9 suffering further injury to the IOM (Table 9, Table 10, Table 11, Table 12, and Table 14).

Wei et al. (2010) intended to re-create syndesmotic ankle sprains under forceful external foot rotation but found mostly fibula fractures (4 of 10) and PTaFL avulsions off the fibula (4 of 10), with only 1 specimen sustaining a medial ligament injury. In the external rotation experiments of Markolf et al. (1989) 12 of 19 specimens experienced fibula fractures, 1 experienced calcaneus fracture, 1 subtalar dislocation, and 5 lateral ligament failures. Similarly, Michelson et al. (1997) also generated a high occurrence of fibula fractures (15 of 30 specimens), a low occurrence of deltoid ligament injury (4 of 30), and a low occurrence of ATIFL injury (7 of 30). In contrast, in this chapter, ATIFL injuries were more common than fibula fractures, occurring in 9 of 17 specimens tested, compared to 7 of 17 experiencing fractures (Table 14). 
These discrepancies in injury generation could be attributed to differences in rotation magnitudes (i.e., current study rotated tibia internally up to $90^{\circ}$ ) and boundary conditions. Markolf et al. (1989), Michelson et al. (1997), and Wei et al. (2012b, 2010) all rigidly constrained the fibula to the tibia and either rigidly fixed the foot or placed the foot in an unspecified, initially taped position. Rigidly constraining the tibia to the fibula artificially constrains the fibula in a manner that limits the natural occurrence of diastasis in the tibiofibular syndesmosis. When the ankle is forced into external rotation (under vertical preload), the talar dome wedges against the medial and lateral malleoli, forcing the syndesmosis to spread apart. Under natural boundary constraints with the fibula only constrained by ligament attachments, this diastasis continues to build until the distal syndesmotic ligaments and IOM rupture. When the fibula is unnaturally fixed to the tibia, however, any attempt to force the syndesmosis into diastasis also forces the fibula into bending (constrained by the attachment to the tibia). This artificial bending stress in the fibula builds faster, presumably, than diastatic strain in the syndesmotic ligaments, and, as a result, the failure mode changes from syndesmotic ligament injury (as would be expected in athletes on the field) to artefactual fracture in the fibula. Thus, this highlights the importance of re-creating realistic boundary conditions (i.e., unconstrained fibula) when studying injury mechanics in the ankle.

In addition to the boundary conditions on the tibia and fibula, care is also needed to ensure boundary conditions on the foot are also appropriate for studying ankle injury. Reduced constraint of the foot permits leg deformation (tibiofibular diastasis), foot deformation (arch flattening), and foot translation. Each of these natural responses of the lower extremity are necessary to accurately recreate syndesmotic ankle sprains (Lin et al., 2006; Williams et al., 2007). Previous experiments constrained the tibia and fibula together and rotated these coupled bones over a rigid foot (Markolf et al., 1989; Michelson et al., 1997; Wei et al., 2010). Others permitted fibula motion over a fixed foot (Xenos et al., 1995) or constrained the foot with athletic tape (Wei et al., 2012b), thus creating an artificially restrictive boundary condition at the foot. Allowing natural fibula motion and foot translation affects bony kinematics and ligament recruitment in the ankle during foot rotation (Chapter 2), likely affecting resulting injury patterns and propagation sequences.

Another possible contributing factor to the differences in injury generation between the current study and previous experiments is the difference in age and size of tested cadavers. The non- 
senescent, sizable cadavers (Table 6) tested in the current study (average age at death, 47 years; average height and weight, $178.1 \mathrm{~cm}$ and $94.4 \mathrm{~kg}$ ) played an important role in replicating the mostly ligamentous syndesmotic injury scenario seen among the athletic population. Prior studies used older subjects, e.g. age ranged from 49 to 85 years (Markolf et al., 1989), 60 to 80 years (Michelson et al., 1997), or 56 years on average (Wei et al., 2012b). Bone deterioration with aging could have contributed to more bony fractures rather than soft tissue rupture in those studies.

This chapter reports full injury diagnoses and a method for determining ankle injury event sequences, by an expert panel with acknowledgment of confidence and uncertainty, in external foot rotation experiments (Table 13; Figure 22 and Figure 23), which is the first to the author's knowledge. Previous experiments (Markolf et al., 1989; Michelson et al., 1997; Wei et al., 2012b, 2010) simply report documented injuries, often limited to one observed injury, but do not report a sequence of injury initiations and propagations throughout the tests. Despite the deficiencies, the methods used by previous studies informed the loading protocol for the study in this chapter so that a full injury sequence could be determined.

Michelson et al. (1997) rotated all specimens up to $90^{\circ}$ (same as the current study) and recorded the measured reaction torque relative to applied rotation. Torque at failure is reported between 10 and $55 \mathrm{~N}-\mathrm{m}$, however the current study measured reaction moments about the tibia on the order of 60 to 110 N-m (maximum reaction moment was 110 N-m during test on Specimen 752R; Figure 22). Presumably, this difference in measured reaction kinetics can be attributed to the overly constrained testing conditions employed by Michelson et al. (1997) and their testing on older cadavers, where the overly constrained older cadaver legs failed at artificially lower torque values compared to the measured moments in the current study. Wei et al. (2012b, 2010) plot measured torque against applied external rotation and indicate "failure" by a single drop in torque during the loading phase. Rotation was applied in increments until a drop in torque was measured and specimens were then offloaded. Failure torques reported by Wei et al. (2012b, 2010) are similar in magnitude (approximately $90 \mathrm{~N}-\mathrm{m}$ ) as those measured in the current study (max. $110 \mathrm{~N}-\mathrm{m}$ during test on Specimen 752R; Figure 22), however only one injury was documented per specimen, which cannot depict all the possible injuries athletes may suffer during syndesmotic ankle sprains on the field. Markolf et al. (1989) does acknowledge further ligament tears following the fibula 
fractures observed at failure during testing as more applied torque is resisted post-fracture. However, indication of specific ligament injuries, relative to applied torque or foot rotation, after these fractures are not reported. Similarly, the current study found that multiple injuries happen throughout the entire rotation loading phase, often without or prior to a catastrophic drop in moment. Ligaments failed both pre- and post-fracture of the tibia and fibula such that deltoid, ATiFL, and IOM failures occurred concomitant to fibular fractures. These concomitant ligament injuries are now reported, in this chapter, relative to external foot rotation in addition to the ankle failure injuries from catastrophic fibula or tibia fractures. Therefore, though the loading protocols of previous studies informed the current study, such that reaction kinetics and kinematics were measured throughout a continuous applied rotation cycle $\left(90^{\circ}\right.$ of internal tibia rotation), a full determination of the propagation and sequence of diagnosed injuries was implemented in this chapter. This ensures a proper classification of syndesmotic injury scenarios with reaction kinetics and relative bone kinematics measured throughout applied rotation and used to determine an event sequence for all diagnosed injuries, from which injury timing relative to external foot rotation can be ascertained.

Previous studies have investigated the occurrence of syndesmotic injury in varying foot postures by combining external rotation with initially everted feet (Wei et al., 2012b) and with initially inverted feet combined with ankle dorsi/plantarflexion (Michelson et al., 1997). However, this chapter reports syndesmotic injuries in varying angles of initial foot flexion alone combined with external foot rotation, which is the first to the author's knowledge to do so. Close investigation of diagnosed injury incidences (Table 14) and injury event sequences (Table 9, Table 10, Table 11, and Table 12) suggests that foot flexion has an effect on syndesmotic injury occurrence in external foot rotation. In the neutral ankle flexion, syndesmotic injury was observed in 5 of 9 specimens. However, when combining external foot rotation with initial dorsiflexion, syndesmotic injury occurred in all specimens (4 of 4). When external rotation was combined with initial plantarflexion, syndesmotic injury occurred in no specimens. This result suggests that plantarflexion might be protective of the syndesmosis, shifting the injury to other ankle ligaments such as the superficial and deep deltoid complexes or lateral ligaments (ATaFL, CFL, PTaFL). 
Though not observed in all specimens tested, a possible injury propagation sequence for syndesmotic ankle sprains was observed in some specimens. Injuries began in the medial ligaments, and then propagated into either the syndesmotic or lateral ligaments, depending on foot flexion. For the neutral initial flexion posture, just over half of the specimens (5 of 9) had propagation of medial injury into the syndesmosis, whereas further medial or concomitant lateral injuries were caused in the other neutral specimens (4 of 9), presumably offloading the syndesmosis. However, for initially dorsiflexed specimens, injuries started in either the medial or syndesmosis regions of the ankle with further propagation into the syndesmosis (IOM and fibula fracture) later in the rotation cycle. For initially plantarflexed specimens, injuries started in either the medial or lateral regions of the ankle with further propagation into those regions, thus offloading the syndesmosis. Therefore, from these injury incidence results, the talus sufficiently wedged into the ankle mortise during applied external foot rotation to induce syndesmotic injury in the initially neutral and dorsiflexed legs. However, this was not the case for plantarflexed legs where the talus did not wedge into the ankle mortise, even under the same preload and imposed external rotation, to induce syndesmotic injury. This suggests that the talar dome is sufficiently rotated out of the ankle mortise when legs are placed in plantarflexion before external foot rotation is imposed such that tibiofibular diastasis does not occur and the syndesmotic ligaments are protected.

Determining the injury sequence under external rotation helps elucidate the possible injury combinations that may be associated with a syndesmotic injury scenario. As deltoid injuries were common (occurred in 15 of 17 specimens; Table 14) across all of the loading scenarios, these results suggest that it may be pertinent to suspect deltoid injuries in any clinical situation where syndesmotic ankle injury is indicated. The injury sequence information determined in this chapter should inform future clinical classifications of syndesmotic ankle sprains, with the ultimate goal of improving future care, recovery, and prevention methods. Additionally, from the determined injury sequences, a classification of injury timing (relative to applied external foot rotation) is possible. This injury timing can be used to confirm the hypothesized injury mechanism for syndesmotic ankle sprains and determine injury occurrence intervals, caused by external foot rotation, for the syndesmotic, deltoid, and lateral ankle regions. Furthermore, analysis of other 
contributing factors (e.g., tibiofibular diastasis) relative to syndesmotic injury during experiments investing hyper-rotation of the foot is possible with this injury timing and sequence data. 


\section{Chapter 4: Syndesmotic Injury Occurrence and Tibiofibular Diastasis}

\subsection{Introduction}

Injury biomechanics research provides a necessary link between clinical injuries and mechanical function of human anatomy. At failure, the mechanical function of anatomical structures manifests as an injury tolerance based on a parameter's (e.g., measurable stimuli) utility for causing injury (Kent and Funk, 2004). For sprains to the distal tibiofibular syndesmosis, this measurable stimulus is hypothesized as external rotation of the foot (Anderson et al., 2010; Bloemers and Bakker, 2006; Guise, 1976; Lin et al., 2006; Mait et al., 2017b; Norkus and Floyd, 2001; Teramoto et al., 2008; Williams et al., 2007; Wolfe et al., 2001).

As the proposed mechanism of injury for syndesmotic ankle sprains, external foot rotation induces tibiofibular diastasis as the talus rotates within the ankle mortise (Bloemers and Bakker, 2006; Guise, 1976; Norkus and Floyd, 2001; Teramoto et al., 2008). This diastasis forces the fibula laterally and posteriorly from its articular surface with the tibia (Bonnin, 1970; Edwards and DeLee, 1984; Lin et al., 2006; Rose et al., 2002; Williams et al., 2007), potentially injuring the stabilizing ligaments in the syndesmosis such as the ATiFL, PTiFL, and IOM (Anderson et al., 2010; Bloemers and Bakker, 2006; Boytim et al., 1991; Lin et al., 2006; Norkus and Floyd, 2001; Williams et al., 2007; Wolfe et al., 2001).

Tibiofibular diastasis is a subtle kinematic phenomenon where, compared to the uninjured leg, the clear space between the tibia and fibula increases by as little as 1 to $6 \mathrm{~mm}$ during syndesmotic ligament injury (Bonnin, 1970; Husfeldt, 1937; Rammelt et al., 2008; Sclafani, 1985; Williams et al., 2007). Furthermore, after sectioning of the ATiFL, tibiofibular diastasis amounted to only 2.3 $\mathrm{mm}$ (Xenos et al., 1995). Moreover, diagnosis of this kinematic phenomenon with radiographs is unreliable (Hopkinson et al., 1990; Ogilvie-Harris et al., 1994), where syndesmotic injury can be present without the prevalence of frank diastasis (Edwards and DeLee, 1984; Nussbaum et al., 2001). This presents a challenge for clinicians diagnosing syndesmotic ankle sprains preoperatively. 
Though other experiments have studied the effects of forceful external foot rotation (Markolf et al., 1989; Michelson et al., 1997; Villwock et al., 2009; Wei et al., 2012b, 2010; Xenos et al., 1995), to the authors' knowledge, tibiofibular kinematic interactions have not been documented during external foot rotation tests in varying ankle flexion postures. Also, these studies did not determine a relationship between a measurable stimulus and syndesmotic ankle sprain occurrence for legs positioned in various ankle flexion postures.

Based on the hypothesized injury mechanism, a kinematic predictor of injury was assumed (external foot rotation) in this chapter. To focus on this kinematic predictor, muscle forces were not considered. Instead, as detailed in Chapter 3, a compressive load was applied to all specimens through the tibia, intending to simulate weight-bearing during typical dynamic play situations (Wei et al., 2012b, 2012a, 2010).

Based on the results from the injury tests detailed in Chapter 3, the work in this chapter intended to determine injury occurrence intervals, relative to input leg kinematics (external foot rotation), within the medial, lateral, and syndesmotic regions of the ankle (Objectives 2 and 3). Also, this chapter aimed to quantify fibular motions relative to the tibia before, during, and after syndesmotic injuries (Objective 3). External foot rotation and tibiofibular diastasis are classified as closely related clinically, such that the external load on the foot (external rotation) causes the internal load in the ankle joint (tibiofibular diastasis), thus inducing syndesmotic injury as the fibula displaces from the tibia. Therefore, to link these two related kinematic phenomena, fibular displacements (relative to the tibia) in all directions (i.e., anterior-posterior, medial-lateral, and inferior-superior) were investigated to discern which displacements contribute most to tibiofibular diastasis. Differences in the tibiofibular rotations before, during, and after syndesmotic injury were also quantified to discern the influence of fibula rotations on tibiofibular diastasis. Injury occurrence intervals, fibular displacements, and tibiofibular rotation differences were quantified in varying initial ankle postures (neutral, dorsiflexion, and plantarflexion) to discern their sensitivity to ankle flexion posture (Objectives 2 and 3). The reported injury intervals should inform future countermeasure designs (e.g., footwear and ankle braces) aimed at mitigating syndesmotic injuries (Objective 4), and the tibiofibular kinematic data reported in this chapter will act as a resource for future clinical use during diagnoses of syndesmotic ankle sprains (Objective 3). 


\subsection{Methods}

The data analyzed in this chapter were all sourced from the experiments detailed in Chapter 3; please see Section 3.2 for full methodologies regarding specimen preparation, experimentation, post-test necropsies, and injury event identifications.

\subsubsection{Tibiofibular Diastasis Determination}

As detailed in Chapter 3 (Section 3.2.1), a multi-camera optoelectronic motion capture system (Hardware: Vicon T-series; Software: Nexus 2.2; Vicon, CA, USA) was used to measure bony kinematics within the ankle during testing. Motions of the fibula, tibia, and calcaneus were tracked throughout the rotation cycle. Bony motions, calculated with rigid-body transformations derived from CT scans (Lessley et al., 2011; Shaw et al., 2009), are reported in a geometrically defined tibia-based coordinate system (Figure 8). The coordinate axes of the fibula (origin in the centroid of the lateral malleolus) and calcaneus (origin at bone centroid) were defined initially parallel to those of the tibia coordinate system in a neutral ankle posture (Figure 8).

With bone coordinate systems defined rigorously using bone geometry and anatomical landmarks, relative motion between the foot and ankle bones caused by the input motion of the tibia can be quantified. This is advantageous for quantifying tibiofibular kinematic interactions (tibiofibular diastasis) before, during, and after syndesmotic injury. Clinicians traditionally define tibiofibular diastasis as a lateral displacement of the fibula relative to the tibia, with posterior fibular displacement possibly contributing to tibiofibular diastasis (Bonnin, 1970; Edwards and DeLee, 1984; Lin et al., 2006; Rose et al., 2002; Williams et al., 2007). However, arbitrarily restricting tibiofibular diastasis definitions to one component or another of fibula motion relative to the tibia does not fully quantify tibiofibular kinematic interactions during and after syndesmotic injury. Instead, with the six degree-of-freedom tibia and fibula kinematics measured in Chapter 3, a displacement vector $(<\mathrm{Dx}, \mathrm{Dy}, \mathrm{Dz}\rangle)$ relating the position of the fibula origin relative to the tibia origin can be defined. This displacement vector has three components of linear displacement (translations) of the fibula's origin along the tibia's axes such that $\mathrm{Dx}=\Delta \mathrm{x}, \mathrm{Dy}=\Delta \mathrm{y}$, and $\mathrm{Dz}=\Delta \mathrm{z}$ (calculated with Equations (4), (5), and (6)). Since the fibula is continuously translating relative to 
the tibia throughout applied rotation, this displacement vector quantifies all fibula translations, along the $\mathrm{x}, \mathrm{y}$, and $\mathrm{z}$ axes of $\mathrm{CS}_{\text {Tibia, }}$ at any time during the rotation cycle. This allows for all components of fibula displacement to be measured before, during, and after syndesmotic injury instead of just one component during diagnosis on post-injury radiographs (Hopkinson et al., 1990; Ogilvie-Harris et al., 1994).

To illustrate the contributions of fibular displacement in all three directions along the tibia's axes, three-dimensional reconstructions of the tibia and fibula were generated using Mimics 18.0 at the beginning of a neutral test (Chapter 3) and after syndesmotic injury (Figure 25). The white arrows indicate a combination of inferior, lateral, and posterior fibula translation relative to the tibia postsyndesmotic injury. As a reminder, for the coordinate systems defined in Figure 8, inferiorsuperior linear displacement (translation) is $+\Delta \mathrm{z}$ and $-\Delta \mathrm{z}$, anterior-posterior translation is $+\Delta \mathrm{x}$ and $-\Delta \mathrm{x}$, and medial-lateral is $+\Delta \mathrm{y}$ and $-\Delta \mathrm{y}$ for left legs but $-\Delta \mathrm{y}$ and $+\Delta \mathrm{y}$ for right legs. Since tibiofibular kinematic interactions are composed of a combination of inferior-superior, mediallateral, and anterior-posterior displacements throughout the rotation cycle, the magnitude of the displacement vector between the fibula and tibia origins $\left(\sqrt{ }\left(\Delta \mathrm{x}^{2}+\Delta \mathrm{y}^{2}+\Delta \mathrm{z}^{2}\right)\right)$ was calculated throughout the rotation cycle. The magnitude of this displacement vector takes into account the three-dimensional translations of the fibula relative to the tibia throughout the rotation cycle, instead of relying on one component of fibula translation post-injury. With the origins of the tibia and fibula defined based on geometry and anatomical landmarks, each displacement vector is defined for each specimen and accounts for anatomical variabilities. Therefore, this displacement vector can be compared across the sample of specimens.

The fibula also rotates continuously relative to the tibia during applied rotation. Therefore, similar to quantifying the fibula displacement relative to the tibia before, during, and after syndesmotic injury, the difference between tibia and fibula yaw, pitch, and roll angles were also quantified throughout the rotation cycle. This difference was calculated simply, for every time step during the rotation cycle, as fibula rotation minus tibia rotation for each of the three rotation components. These rotations were quantified relative to the global laboratory coordinate system since the global axes were initially coincident to the tibia's local axes. As the tibia is rotated constantly, the fibula rotates independently, since it is not connected rigidly to the tibia and is instead connected by 
ligamentous constrains, as it reacts to the input tibia rotation. Therefore, to ascertain the degree to which the syndesmosis joint opens rotationally, the difference between tibia and fibula rotation in the global coordinate system must be quantified. Quantifying fibula rotations relative to the tibia coordinate system would not discern independent rotations between the two bones, instead continuously changing tibia rotations would influence fibula rotations. These independent rotations add to tibiofibular diastasis inducing syndesmotic injury. Therefore, tibiofibular diastasis is considered to be a combination of the fibula displacement vector magnitude and fibula rotation difference from tibia rotation.

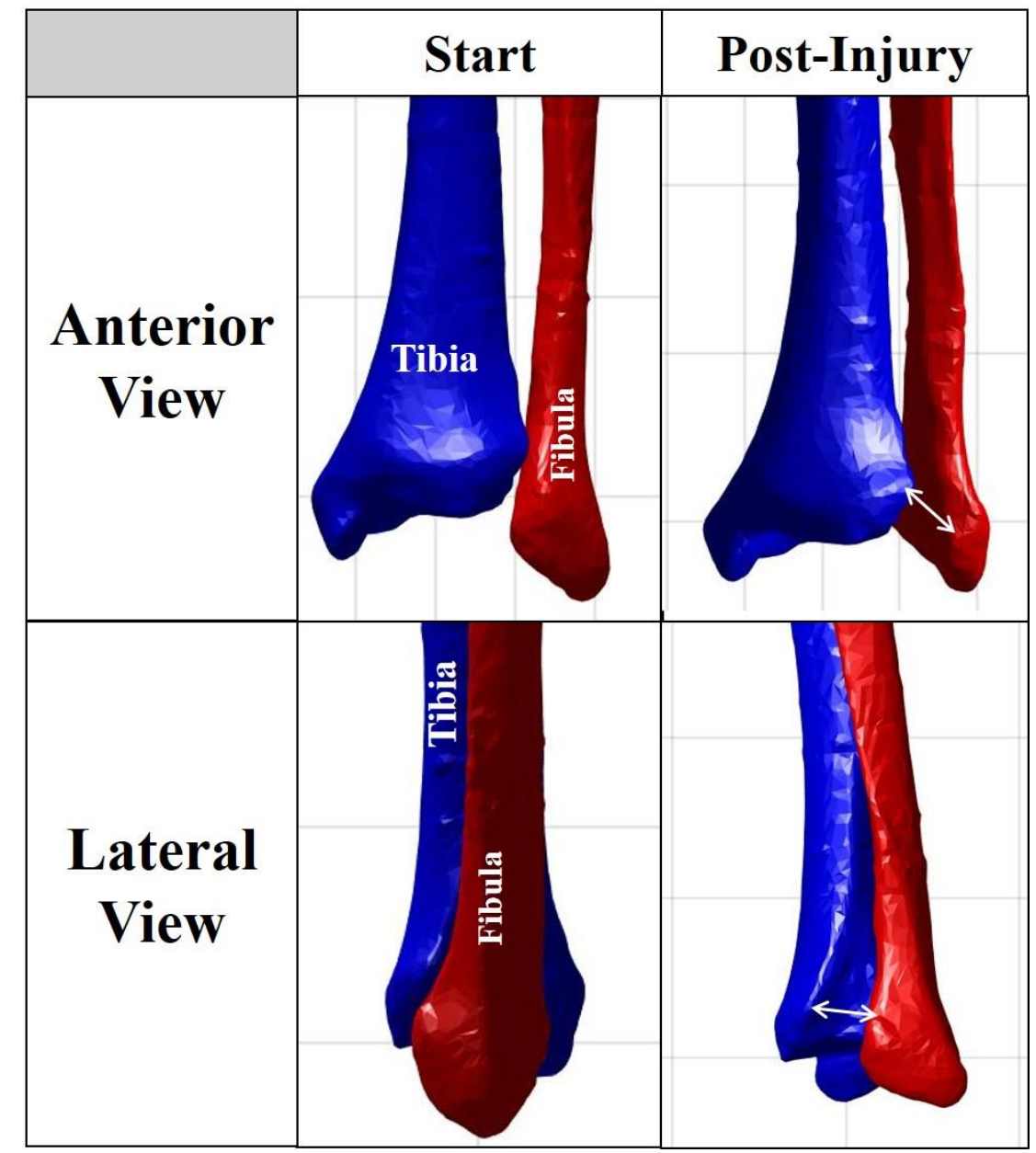

Figure 25: Illustration of components from fibula displacement vector (in tibia coordinate system) in three-dimensional reconstructions of tibia and fibula. Anterior view illustrates (white arrow) the lateral and inferior displacement components and lateral view illustrates the posterior component. 
To determine the relationship between the externally applied injury mechanism (external foot rotation) and the internal (within ankle joint) injury mechanism (tibiofibular diastasis) inducing syndesmotic injury, the components of the fibula displacement vector relative to the tibia and the magnitude of this vector were plotted against external foot rotation. Also, the difference between tibia and fibula rotation (in the global coordinate system) components were plotted against external foot rotation. Ideally, these plots will indicate an increase in fibula displacement and rotation relative to the tibia for the limbs where syndesmotic injury was diagnosed, compared to those limbs diagnosed without syndesmotic injury (Table 9, Table 10, Table 11, and Table 12).

\subsubsection{Ankle Injury Occurrence Interval Determination}

Determining the ankle's tolerance of external foot rotation (ER) for syndesmotic injury in the form of statistical models (e.g., injury risk functions; Eppinger et al., 1999; Kent and Funk, 2004) is difficult for the experimental work and injury diagnoses detailed in this thesis. Injuries are confounded as excessive external rotation (internal tibia rotation of up to $90^{\circ}$ ) is applied such that multiple injuries are caused in each specimen. Discerning a particular injury as a statistically independent event relative to another is difficult as a superficial deltoid injury could predispose the ankle to a subsequent syndesmotic or lateral injury depending on initial ankle flexion posture (Table 9, Table 10, Table 11, and Table 12). This is the difficulty with ligamentous injury in general, and specifically in the ankle joint where load paths are shared across a number of ligaments, in any particular posture, thus an increase in the risk of rupture to one ligament after the rupture of another ligament is always a possibility. Instead, to summarize the data in Table 15, Table 16, Table 17, and Table 18, a range of ER for injuries in the syndesmotic (injuries to ATiFL or IOM or Weber B/C classified fibula fractures; Hughes et al., 1979), medial (injuries to superficial and deep deltoid complexes), and lateral (injuries to ATaFL, CLF, or PTaFL or Weber A classified fibula fracture; Hughes et al., 1979) ankle regions was determined. These ranges of ER indicate an injury occurrence interval such that minimum, median, and maximum ER values were mined from Table 15, Table 16, Table 17, and Table 18 in all initial ankle flexion postures. Comparisons across these initial ankle postures were considered to determine sensitivity of ankle injury occurrences, relative to applied external foot rotation, to ankle posture. 
All intervals of ER were calculated with the uncertainty of the injury event sequence determination (Section 3.2.5; Table 8) taken into account. Only the injury events that were classified, by the expert panel, as certain for diagnosed injury matching were given one value of ER. Ranges of ER for all other injury events, with classifications of probable, possible, and unknown, were also implemented into the injury interval. For example, in Table 16, events 1 and 2 for Run 4 were classified as either injuries to the CFL, PTaFL, or superficial deltoid. The ER values for events 1 and $2\left(35.6^{\circ}\right.$ and $\left.43.2^{\circ}\right)$ were both included in the injury interval for the lateral ankle region among initially neutral specimens, and event 2 was included in the injury interval for the medial ankle region among initially neutral specimens. Where syndesmotic, lateral, or medial injury was not diagnosed in a particular specimen post-test, the maximum ER value, imposed on that specimen, was included in the injury intervals for each initial ankle posture. Including these maximum values takes into account the censoring of injury data where the maximum ER values indicate rightcensored data and the exact or interval ER values for particular injuries in each test indicate uncensored data. With the ER values from Table 15, Table 16, Table 17, and Table 18, a similar interval was determined for the magnitude of the displacement vector of the fibula origin relative to the tibia origin and the fibula and tibia rotation differences among specimens where syndesmotic injury was diagnosed (initially neutral and dorsiflexed specimens only). These intervals were generated for ATiFL, IOM, and Weber B/C fibula fracture syndesmotic injuries only to indicate the tibiofibular diastasis caused, within this particular sample of cadavers, which induced syndesmotic injury (i.e., does not include right-censored data for displacement magnitude or rotation difference). This will allow for direct comparison between the externally applied injury mechanism and the internal (within ankle joint) injury mechanism for syndesmotic ankle sprains.

\subsection{Results}

For comparison across initial ankle flexion postures, the injury intervals, relative to external foot rotation ( $\Delta$ yaw of calcaneus about tibia long-axis) from the injury timing data in Table 15, Table 16, Table 17, and Table 18, for the legs in the initially neutral, dorsiflexed, and plantarflexed postures are given in Figure 26. In the neutral posture, the syndesmotic (i.e., ruptures and avulsions

of the ATiFL or IOM or Weber B and C classified fibula fractures) injury interval was $28.6^{\circ}$ to $80.8^{\circ}$ of ER (minimum to maximum) and $33.5^{\circ}$ to $55.7^{\circ}$ (quartile 1 to quartile 3 ), with a median 
of $45.3^{\circ}$. Recall, syndesmotic injury occurred in only 5 of 9 specimens in the neutral ankle posture sample, therefore the maximum ER value for this injury interval indicates the maximum ER (rightcensored) imposed on a specimen that did not sustain a syndesmotic injury. Deltoid injury (i.e., ruptures and avulsions of the superficial and deep deltoid complexes) in the neutral posture occurred earlier in the rotation cycle compared to syndesmotic injury with a median of $40.1^{\circ}\left(34.8^{\circ}\right.$ to $48.4^{\circ}$ for quartile 1 to quartile 3 ), and minimum to maximum of $20.1^{\circ}$ to $55.7^{\circ}$. Recall, deltoid injury occurred in all nine neutral specimens, therefore the ER values for this injury interval contains all uncensored data. However, lateral injury (i.e., ruptures and avulsions of the ATaFL, CFL, or PTaFL or Weber A classified fibula fractures) occurred later in the rotation cycle compared to syndesmotic injury with a median of $53.2^{\circ}\left(44.3^{\circ}\right.$ to $60.4^{\circ}$ for quartile 1 to quartile 3 ), and minimum to maximum of $28.3^{\circ}$ to $72.9^{\circ}$. Recall, lateral injury occurred in only 6 of 9 specimens in the neutral ankle posture sample, therefore the maximum ER value for this injury interval indicates the maximum ER (right-censored) imposed on a specimen that did not sustain a lateral injury.

For the plantarflexed posture, syndesmotic injury did not occur, but compared to neutral (Figure 26), deltoid injury occurred earlier in the rotation cycle with a median of $28.9^{\circ}\left(21.8^{\circ}\right.$ to $34.1^{\circ}$ for quartile 1 to quartile 3), and minimum to maximum of $16.0^{\circ}$ to $50.7^{\circ}$. Similarly, lateral injury occurred earlier in the rotation cycle during plantarflexion compared to neutral with a median of $25.1^{\circ}\left(22.2^{\circ}\right.$ to $37.4^{\circ}$ for quartile 1 to quartile 3$)$, and minimum to maximum of $20.5^{\circ}$ to $57.3^{\circ}$. For the dorsiflexed posture, compared to neutral (Figure 26), all ankle region injuries occurred slightly later in the rotation cycle. Syndesmotic injury in dorsiflexion occurred with a median of $49.6^{\circ}$ $\left(45.5^{\circ}\right.$ to $56.4^{\circ}$ for quartile 1 to quartile 3 ), with minimum to maximum of $38.7^{\circ}$ to $71.1^{\circ}$. Similarly, deltoid injury in dorsiflexion occurred with a median of $49.1^{\circ}\left(42.8^{\circ}\right.$ to $60.0^{\circ}$ for quartile 1 to quartile 3 ) and minimum to maximum of $26.2^{\circ}$ to $75.4^{\circ}$, and lateral injury occurred with a median of $63.6^{\circ}\left(60.0^{\circ}\right.$ to $66.1^{\circ}$ for quartile 1 to quartile 3$)$ and minimum to maximum of $52.6^{\circ}$ to $70.0^{\circ}$. 

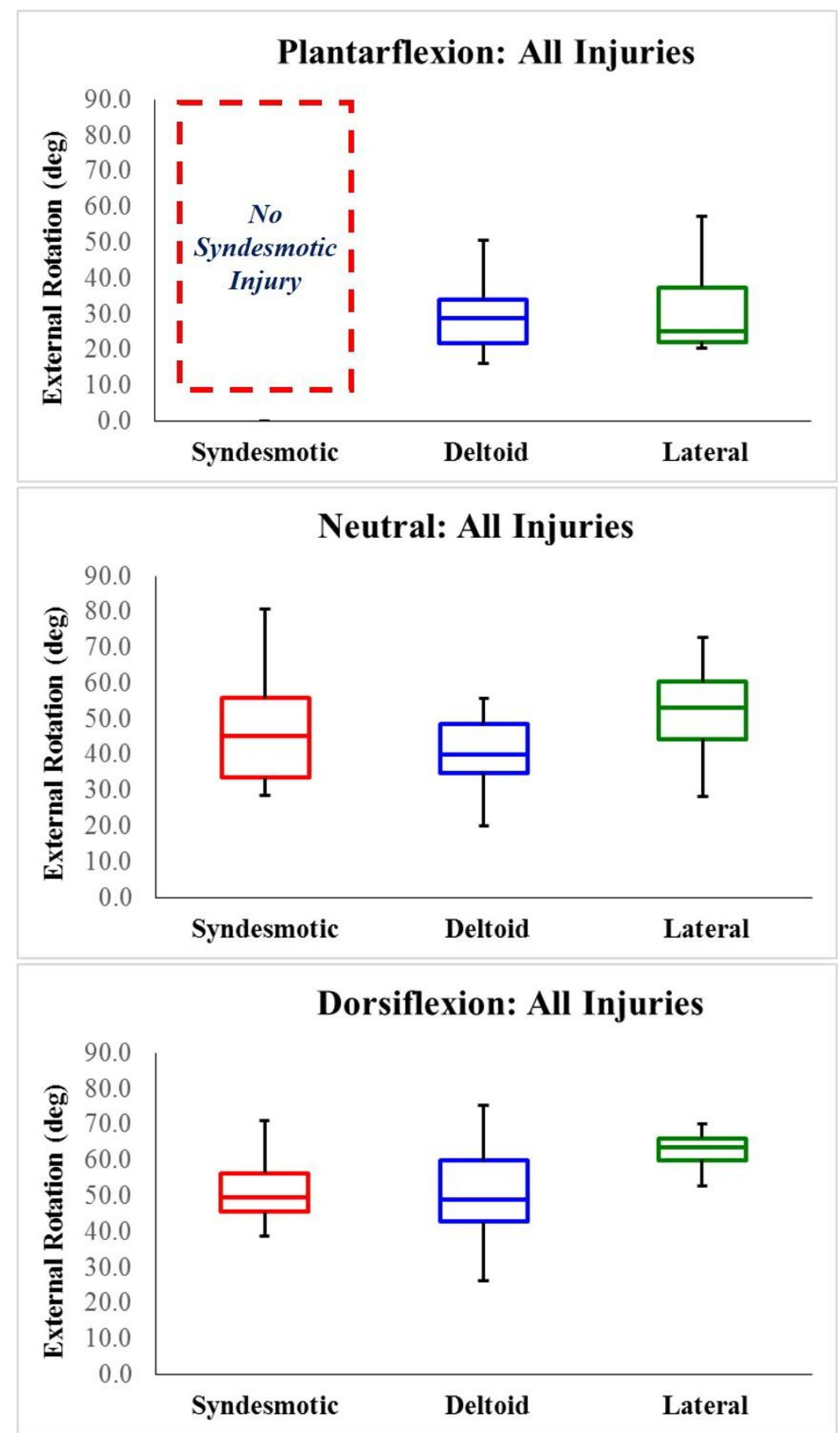

Figure 26: Injury intervals, relative to external foot rotation, for injuries to the syndesmotic (i.e., ATiFL, IOM, and Weber B/C fibula fractures), deltoid (i.e., superficial and deep deltoid complexes), and lateral (i.e. ATaFL, PTaFL, CFL, and Weber A fibula fractures) ankle regions. Sensitivity to initial ankle flexion posture is indicated among initially plantarflexed (top), neutral (middle), and dorsiflexed (bottom) legs. 
To compare the externally applied (external foot rotation) injury mechanism for syndesmotic injury to the resultant internal (within ankle joint) injury mechanism, plots of the components of the displacement vector $(<\mathrm{Dx}, \mathrm{Dy}, \mathrm{Dz}\rangle)$ of the fibula origin along the tibia axes and the vector's magnitude $\left(\sqrt{ }\left(\Delta \mathrm{x}^{2}+\Delta \mathrm{y}^{2}+\Delta \mathrm{z}^{2}\right)\right)$ against external foot rotation (ER) were generated (for loading portion of rotation cycle only) for all seventeen injury test runs (Figure 27, Figure 28, and Figure 29). Figure 27 illustrates the increase in fibula displacement from the tibia as external foot rotation was applied for the non-matched pair leg (Specimen 696R) in the neutral posture. Windows of ER, centered around a syndesmotic injury's timing, are depicted to illustrate the change in fibula displacement before, during, and after syndesmotic injury. In Figure 27, all displacement components, and thus the magnitude of the displacement vector, change during and after the diagnosed ATiFL and IOM injury at $33.5^{\circ} \mathrm{ER}$. More noticeably, for the Weber B fibula fracture at $40.3^{\circ} \mathrm{ER}$, the displacement vector magnitude increases sharply (corresponding sharp decrease in Dx) before the fracture and then decreases steeply as the fracture propagates through the bone. Dx decreases, indicating posterior fibula translation, and the displacement vector magnitude increases throughout the rotation cycle until the fibula fracture occurs. However, the medial-lateral (Dy) and inferior-superior (Dz) translations remain relatively constant throughout the rotation cycle until the syndesmotic injuries occur. 


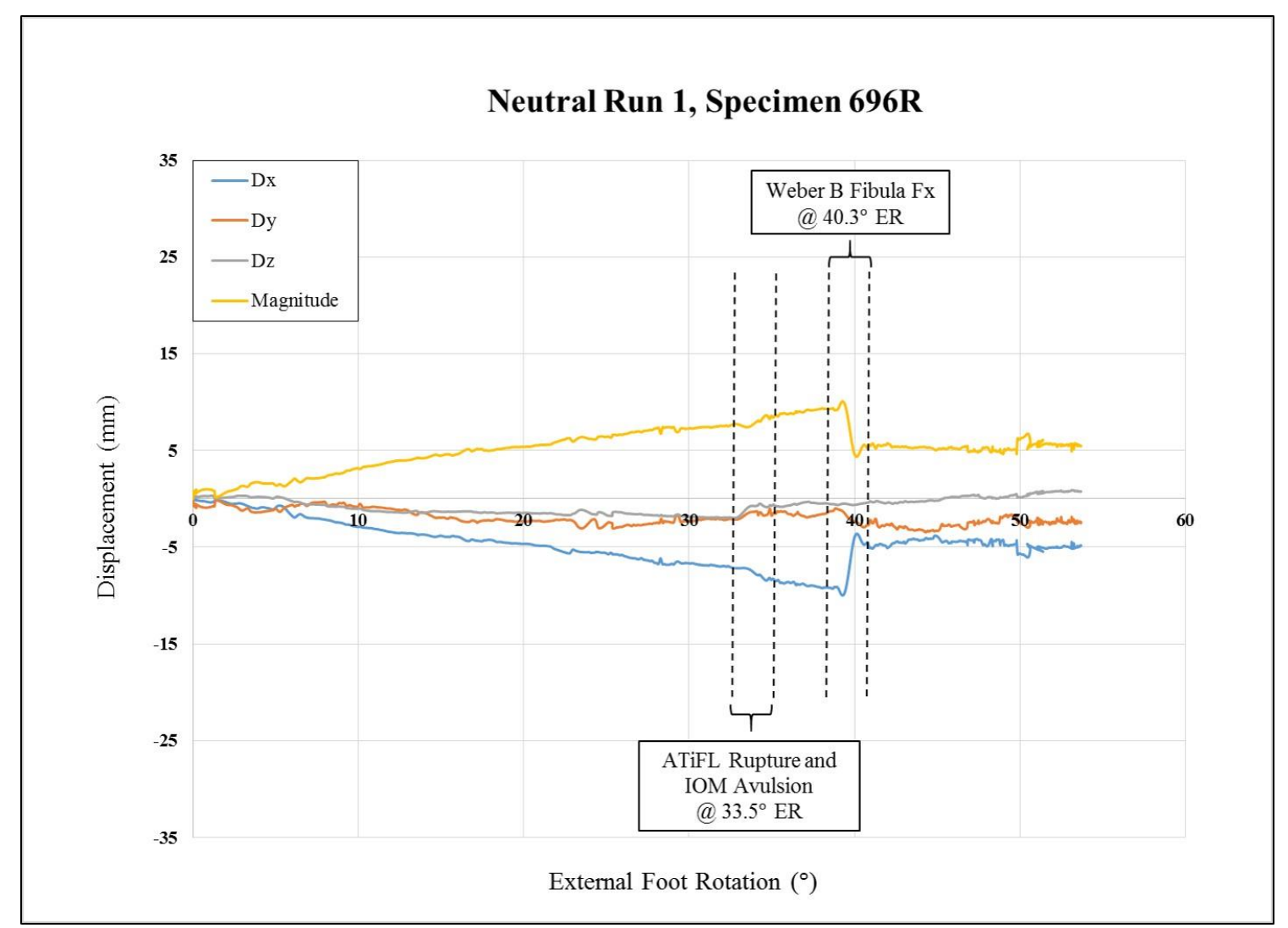

Figure 27: Plot of components of defined fibula displacement vector and magnitude of displacement vector relative to external foot rotation in the non-matched pair initially neutral specimen (Run 1). Intervals of displacement are indicated around the external foot rotation timing for diagnosed syndesmotic injuries.

To compare across initial ankle flexion postures, plots of displacement vector components and magnitude were generated for the remainder of neutral injury tests and the corresponding matchedpair plantarflexed and dorsiflexed legs (Figure 28 and Figure 29). Consistent with Figure 27, the Dx component decreased (posterior fibula translation), with subsequent increase in the vector magnitude, during and after syndesmotic injury as ER was increasingly applied for the neutral and dorsiflexed limbs (Figure 28 and Figure 29). Displacement vector magnitude was typically on the order of 5 to $10 \mathrm{~mm}$ for most neutral and dorsiflexed tests during and after syndesmotic injury, however maximum measured displacement magnitude occurred after an IOM tear $(27.8 \mathrm{~mm})$ in Run 16 on Specimen 757R (Figure 29). In the plantarflexed tests, the displacement vector components were consistently measured at approximately $5 \mathrm{~mm}$ throughout the rotation cycles, which was less than the components measured in the matched-pair neutral tests where displacement increased to almost $25 \mathrm{~mm}$ after syndesmotic injury (Figure 28). 


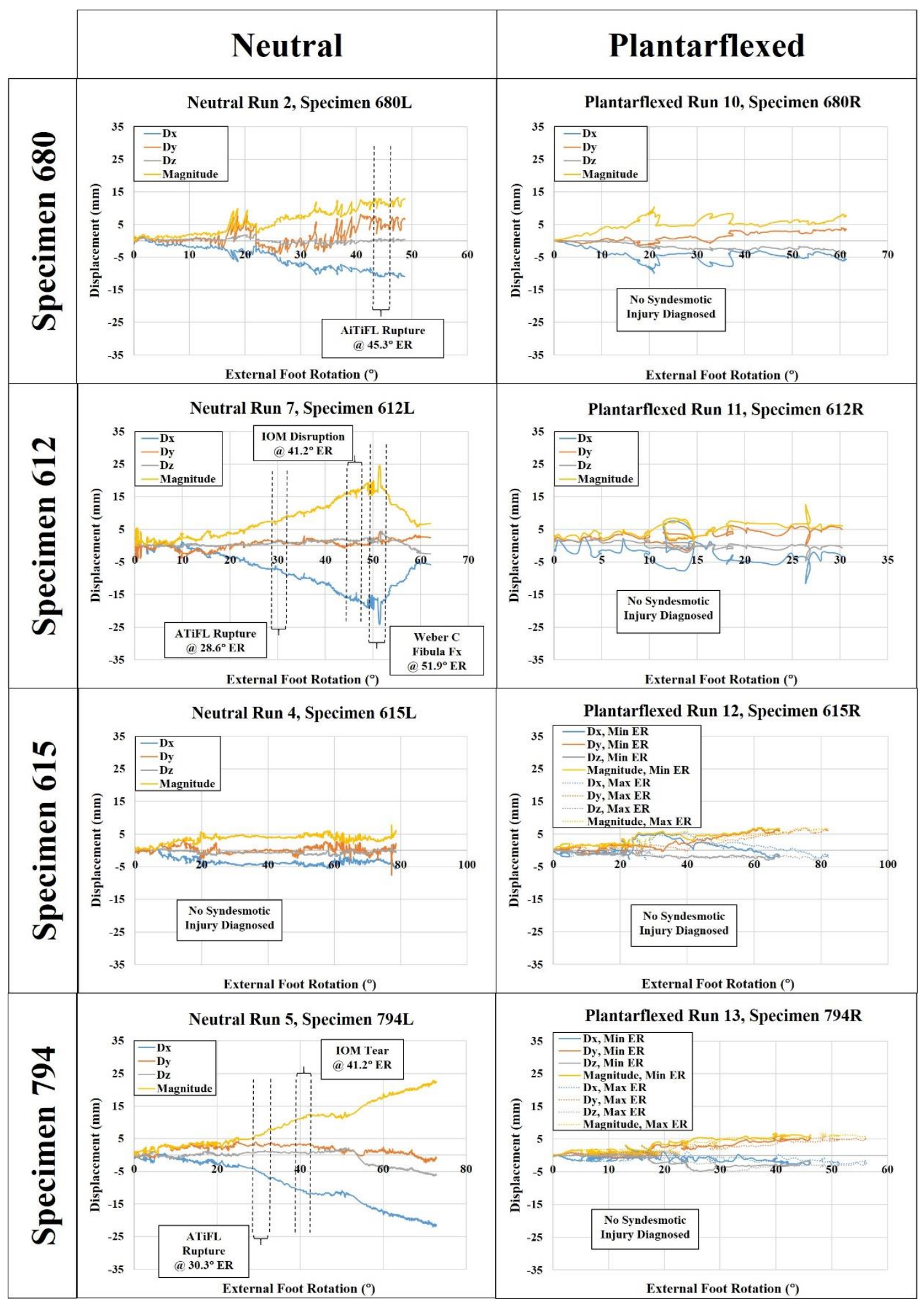

Figure 28: Plot of components of defined fibula displacement vector and magnitude of displacement vector relative to external foot rotation in matched pair specimens for the initially neutral and plantarflexed postures. Intervals of displacement are indicated around the external foot rotation timing for diagnosed syndesmotic injuries. 


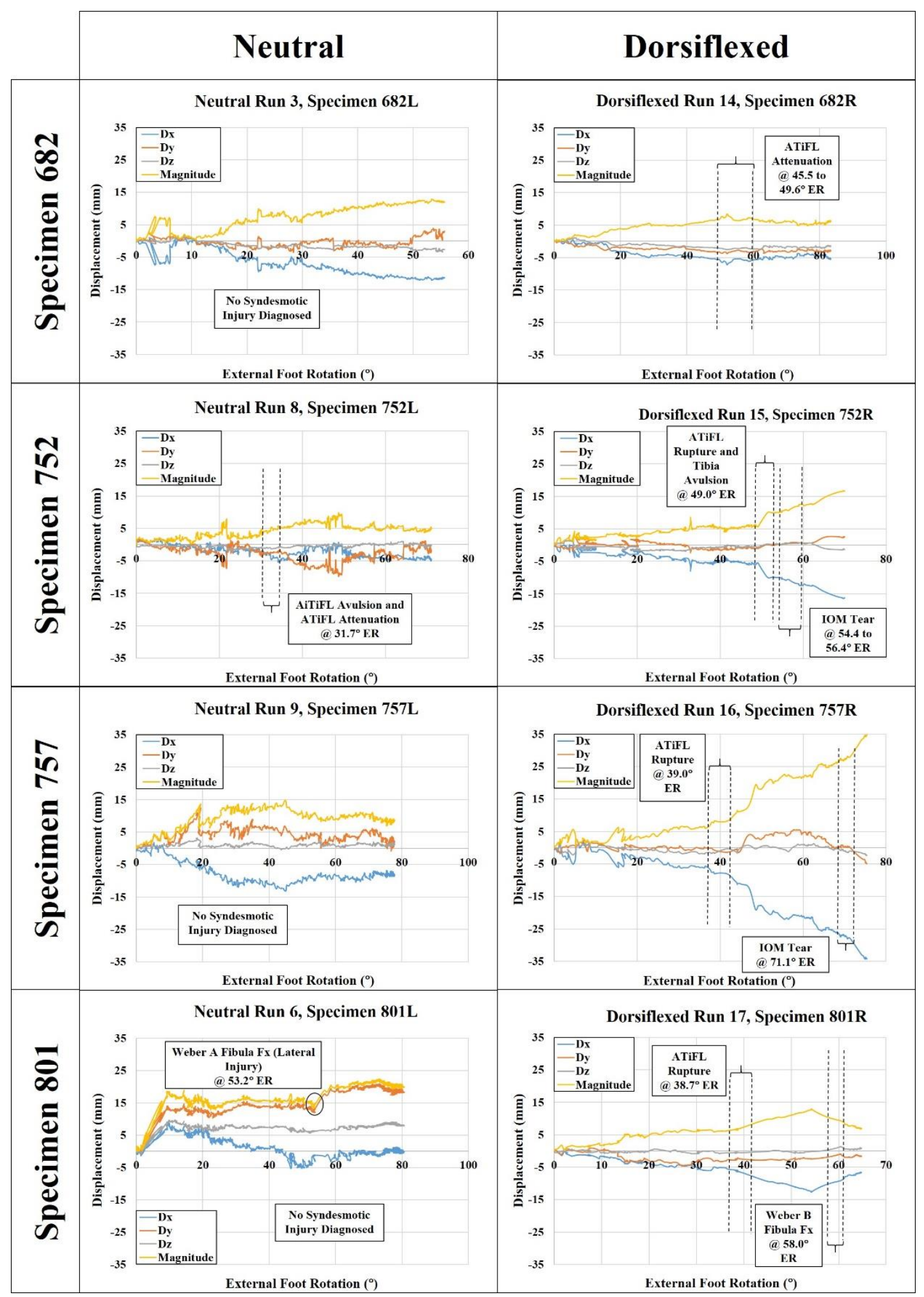

Figure 29: Plot of components of defined fibula displacement vector and magnitude of displacement vector relative to external foot rotation in matched pair specimens for the initially neutral and dorsiflexed postures. Intervals of displacement are indicated around the external foot rotation timing for diagnosed syndesmotic injuries. 
To further compare the externally applied (external foot rotation) injury mechanism for syndesmotic injury to the resultant internal (within ankle joint) injury mechanism, plots of the components of the differences between tibia and fibula rotations (yaw, pitch, and roll with respect to the global coordinate system) against external foot rotation (ER) were generated (for loading portion of rotation cycle only) for all seventeen injury test runs. The difference between fibula and tibia yaw was consistently greater in all tests than the difference between fibula and tibia pitch and roll rotations, thus only the yaw differences are plotted in Figure 30, Figure 31, and Figure 32. Figure 30 illustrates the increase in fibula and tibia yaw difference as external foot rotation was applied for the non-matched pair leg (Specimen 696R) in the neutral posture. Windows of ER, centered around a syndesmotic injury's timing, are depicted to illustrate the change in yaw difference before, during, and after syndesmotic injury. In Figure 30, the fibula and tibia yaw difference increases in conjunction with both syndesmotic injuries, with a sharp decrease after Weber B fibula fracture. 


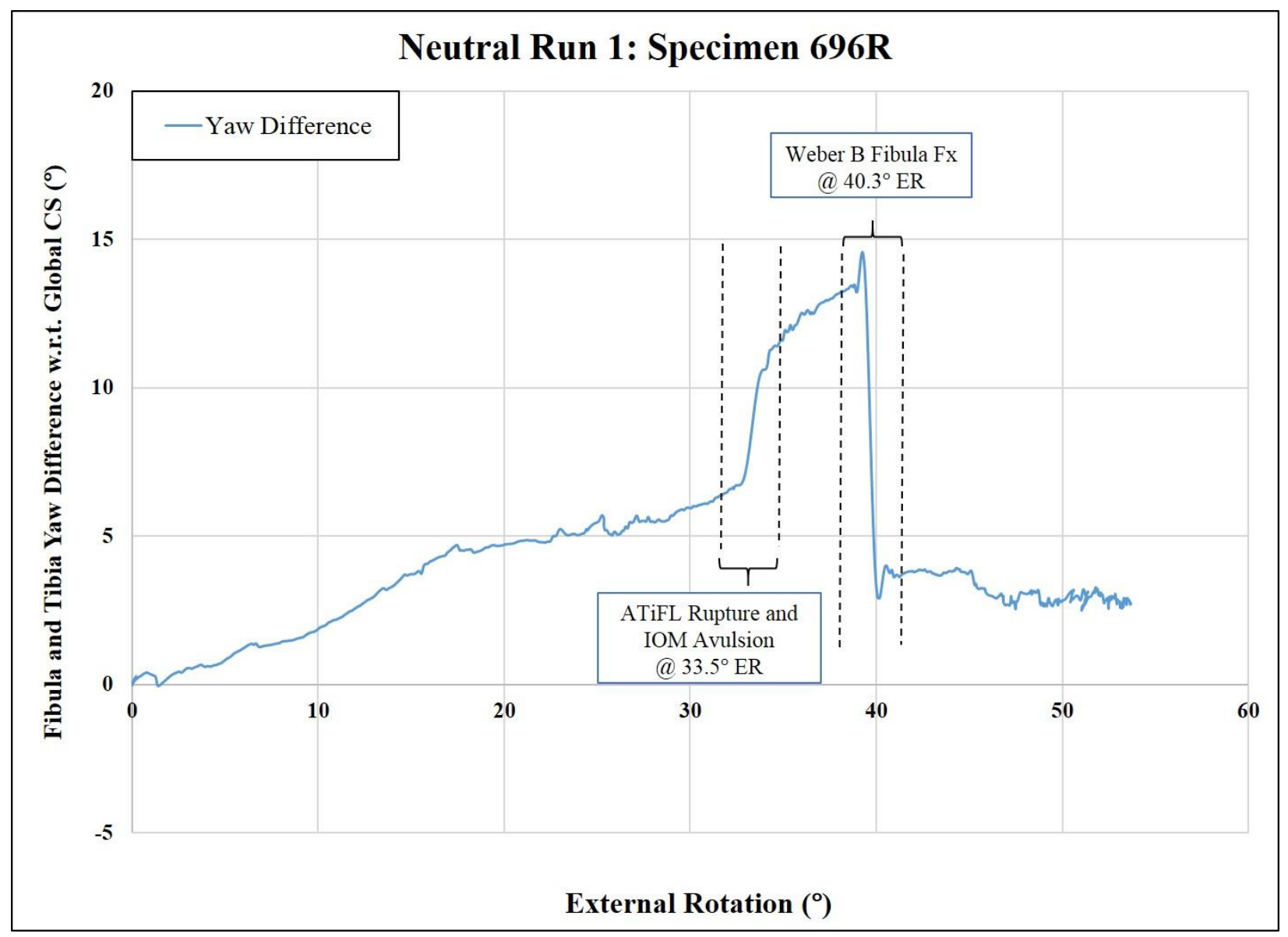

Figure 30: Difference between fibula and tibia yaw in the global coordinate system plotted against external foot rotation in the non-matched pair initially neutral specimen (Run 1). Intervals of rotation difference are indicated around the external foot rotation timing for diagnosed syndesmotic injuries.

To compare across initial ankle flexion postures, plots of fibula and tibia yaw difference were generated for the remainder of neutral injury tests and the corresponding matched-pair plantarflexed and dorsiflexed legs (Figure 31 and Figure 32). Consistent with Figure 30, fibula and tibia yaw difference increased before and during syndesmotic injury as ER was increasingly applied for the neutral and dorsiflexed limbs (Figure 31 and Figure 32), with sharp drops in yaw difference after fibula fracture. In the plantarflexed tests, the yaw difference was consistently measured at approximately less than $10^{\circ}$ throughout the rotation cycles (Figure 31). In the plantarflexed and neutral tests where syndesmotic injury did not occur, the slope of the yaw difference relative to ER plot was relatively constant after, approximately, the initial $20^{\circ}$ of ER (Figure 31 and Figure 32). However, where syndesmotic injury was diagnosed in the dorsiflexed 
and neutral tests, a sharp increase in slope of the yaw difference relative to ER was indicated with some yaw differences reaching to between $30^{\circ}$ and $40^{\circ}$ (Figure 31 and Figure 32).

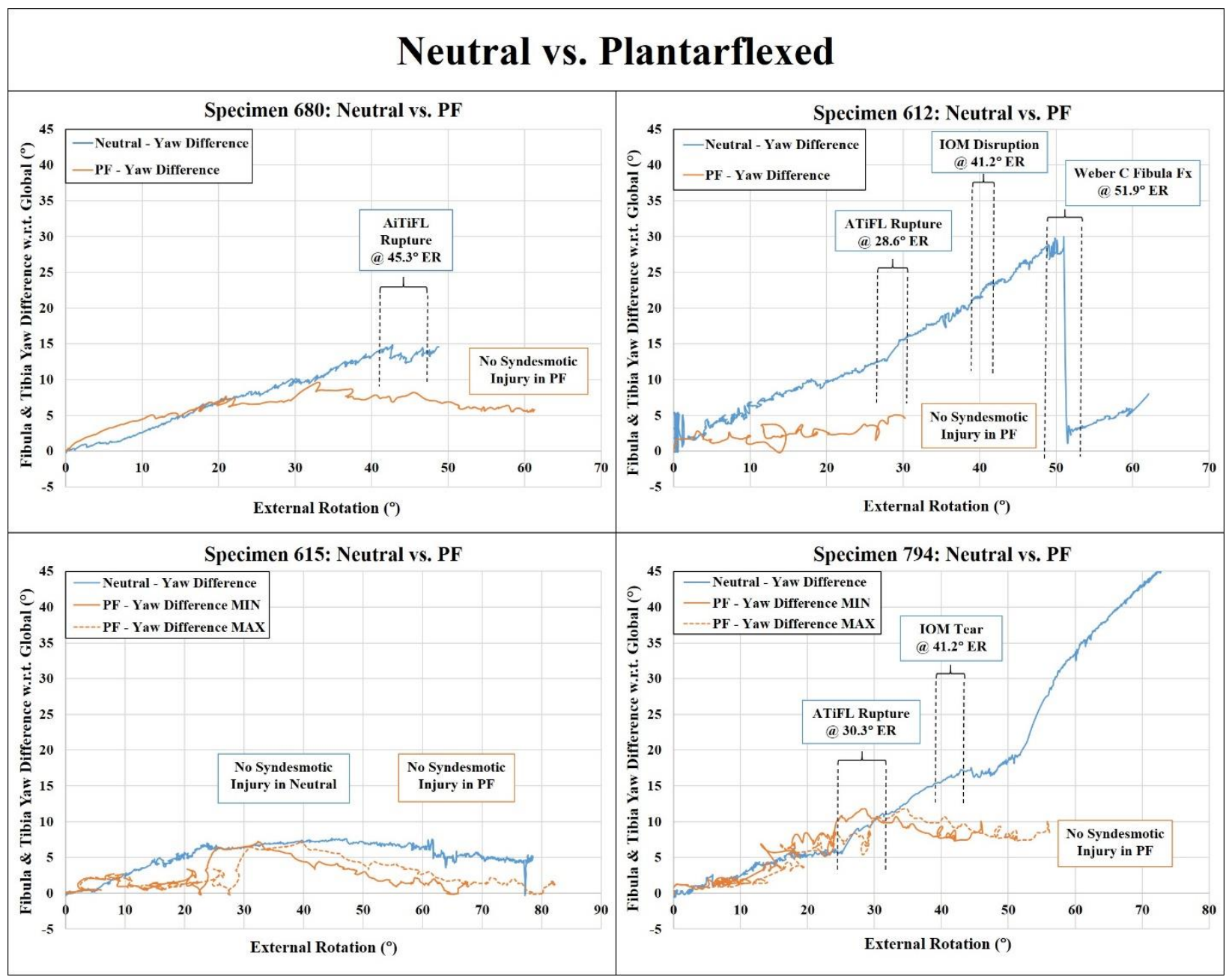

Figure 31: Plot of fibula and tibia yaw difference in the global coordinate system relative to external foot rotation in matched pair specimens for the initially neutral and plantarflexed postures. Intervals of rotation difference are indicated around the external foot rotation timing for diagnosed syndesmotic injuries. 


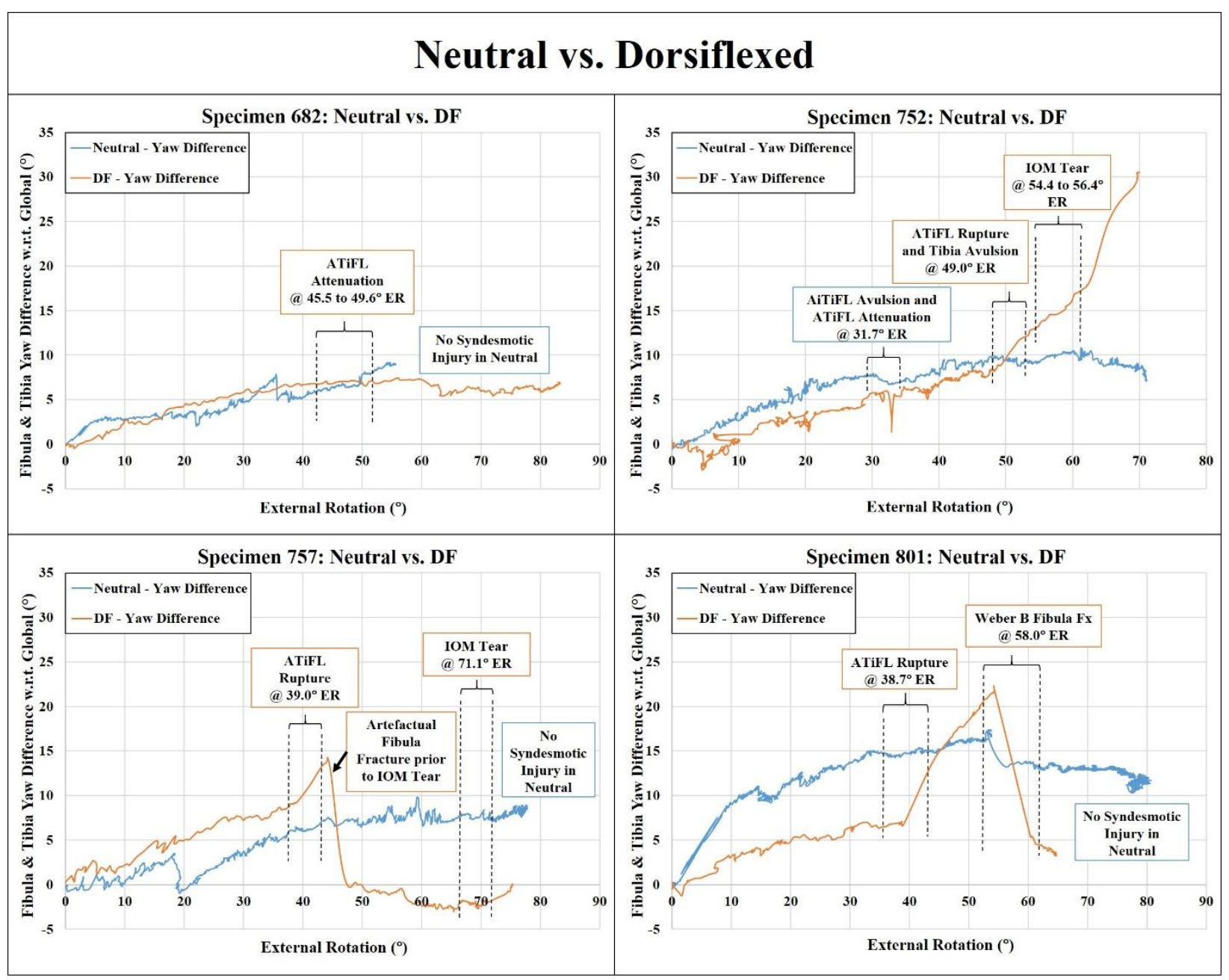

Figure 32: Plot of fibula and tibia yaw difference in the global coordinate system relative to external foot rotation in matched pair specimens for the initially neutral and dorsiflexed postures. Intervals of rotation difference are indicated around the external foot rotation timing for diagnosed syndesmotic injuries.

To summarize the results in Figure 27, Figure 28, and Figure 29, injury intervals relative to fibula displacement vector magnitude and based on injury timing (Table 15, Table 16, Table 17, and Table 18) were generated for the syndesmotic injuries (i.e., ruptures and avulsions of the ATiFL or IOM or Weber B and C classified fibula fractures) in the neutral and dorsiflexed ankle posture tests (Figure 33). Recall syndesmotic injury was not diagnosed in plantarflexed posture tests. In the neutral posture, the ATiFL injury interval for fibula displacement magnitude was 3.8 to 10.9 $\mathrm{mm}$ (minimum to maximum) and 6.1 to $7.4 \mathrm{~mm}$ (quartile 1 to quartile 3), with a median of 7.3 $\mathrm{mm}$. The IOM injury interval in the neutral posture occurred with a median of $11.6 \mathrm{~mm}(9.5$ to 
$14.0 \mathrm{~mm}$ for quartile 1 to quartile 3 ), and minimum to maximum of 7.4 to $16.4 \mathrm{~mm}$. Additionally, the Weber B and C fibula fracture injury interval in the neutral posture occurred with a median of $11.4 \mathrm{~mm}$ ( 8.1 to $14.7 \mathrm{~mm}$ for quartile 1 to quartile 3 ), and minimum to maximum of 4.7 to 18.0 $\mathrm{mm}$.

For the dorsiflexed posture, compared to neutral (Figure 33), ATiFL injury occurred at similar fibula displacement magnitudes with a median of $6.7 \mathrm{~mm}(6.2$ to $6.9 \mathrm{~mm}$ for quartile 1 to quartile 3), and minimum to maximum of 5.7 to $8.2 \mathrm{~mm}$. However, for the dorsiflexed posture, IOM injury occurred at slightly higher fibula displacement magnitudes, compared to neutral, with interquartile range of 10.4 to $19.2 \mathrm{~mm}$ (median of $10.6 \mathrm{~mm}$ ), but minimum to maximum of 10.1 to $27.8 \mathrm{~mm}$. Only one Weber B fracture was diagnosed in the dorsiflexed legs and had a fibula displacement magnitude of $10.3 \mathrm{~mm}$ at injury, with this single injury denoted by a dashed box in Figure $\mathbf{3 3}$. Fibula displacement magnitude increased in both the neutral and dorsiflexed legs from ATiFL to IOM injury (Figure 33). 


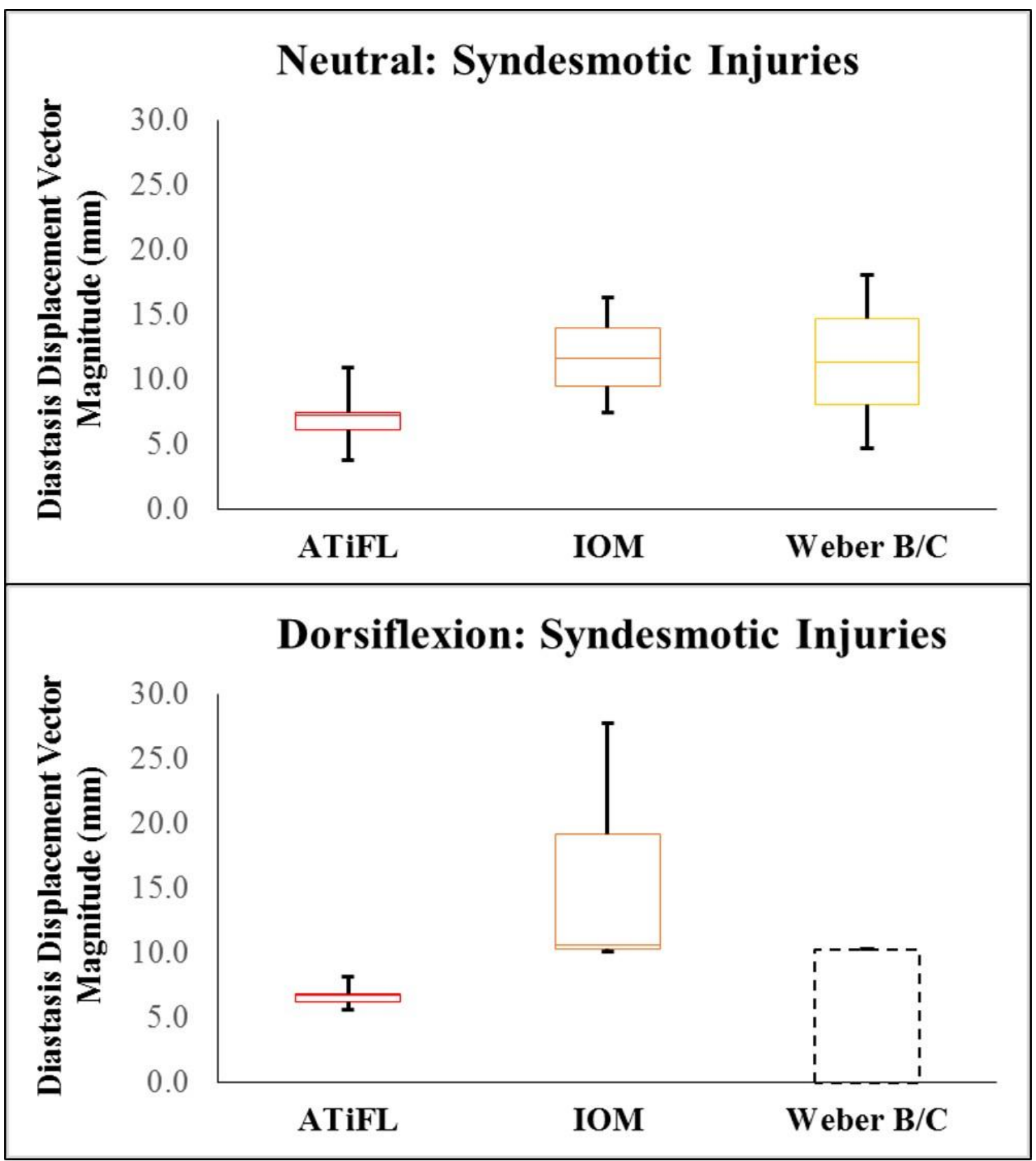

Figure 33: Injury intervals, relative to fibula displacement vector magnitude, for injuries to the syndesmosis (i.e., ATiFL, IOM, and Weber B/C fibula fractures. Sensitivity to initial ankle flexion posture is indicated among initially neutral (top) and dorsiflexed (bottom) legs. Syndesmotic injury did not occur in initially plantarflexed specimens.

To summarize the results in Figure 30, Figure 31, and Figure 32, injury intervals relative to tibia and fibula yaw difference and based on injury timing (Table 15, Table 16, Table 17, and Table 18) were generated for the syndesmotic injuries (i.e., ruptures and avulsions of the ATiFL or IOM or 
Weber B and C classified fibula fractures) in the neutral and dorsiflexed ankle posture tests (Figure 34). In the neutral posture, the ATiFL injury interval for yaw difference was $7.1^{\circ}$ to $13.8^{\circ}$ (minimum to maximum) and $9.4^{\circ}$ to $13.1^{\circ}$ (quartile 1 to quartile 3 ), with a median of $10.2^{\circ}$. The IOM injury interval in the neutral posture occurred with a median of $16.3^{\circ}\left(12.9^{\circ}\right.$ to $21.6^{\circ}$ for quartile 1 to quartile 3 ), and minimum to maximum of $9.4^{\circ}$ to $26.9^{\circ}$. Additionally, the Weber B and $\mathrm{C}$ fibula fracture injury interval in the neutral posture occurred with a median of $22.2^{\circ}\left(18.4^{\circ}\right.$ to $25.9^{\circ}$ for quartile 1 to quartile 3 ), and minimum to maximum of $14.6^{\circ}$ to $29.7^{\circ}$.

For the dorsiflexed posture, compared to the neutral (Figure 34), ATiFL injury occurred at slightly lower tibia and fibula yaw differences with a median of $7.1^{\circ}\left(7.0^{\circ}\right.$ to $8.9^{\circ}$ for quartile 1 to quartile 3 ), and minimum to maximum of $6.9^{\circ}$ to $9.3^{\circ}$. Also, IOM injury occurred at slightly lower yaw differences in the dorsiflexed posture, compared to neutral, with interquartile range of $13.4^{\circ}$ to $14.1^{\circ}$ (median of $13.7^{\circ}$ ), and minimum to maximum of $13.0^{\circ}$ to $14.4^{\circ}$. Only one Weber B fracture was diagnosed in the dorsiflexed legs and had a yaw difference of $22.3^{\circ}$ at injury, with this single injury denoted by a dashed box in Figure 34. For one of the dorsiflexed specimens (Run 16, Specimen 757R: Figure 32), an artefactual fibula fracture occurred before the IOM tear, and therefore was not included in the injury interval for yaw difference since fibula kinematics are unreliable post-fracture. Yaw difference increased in both the neutral and dorsiflexed legs from ATiFL to IOM injury (Figure 34). 


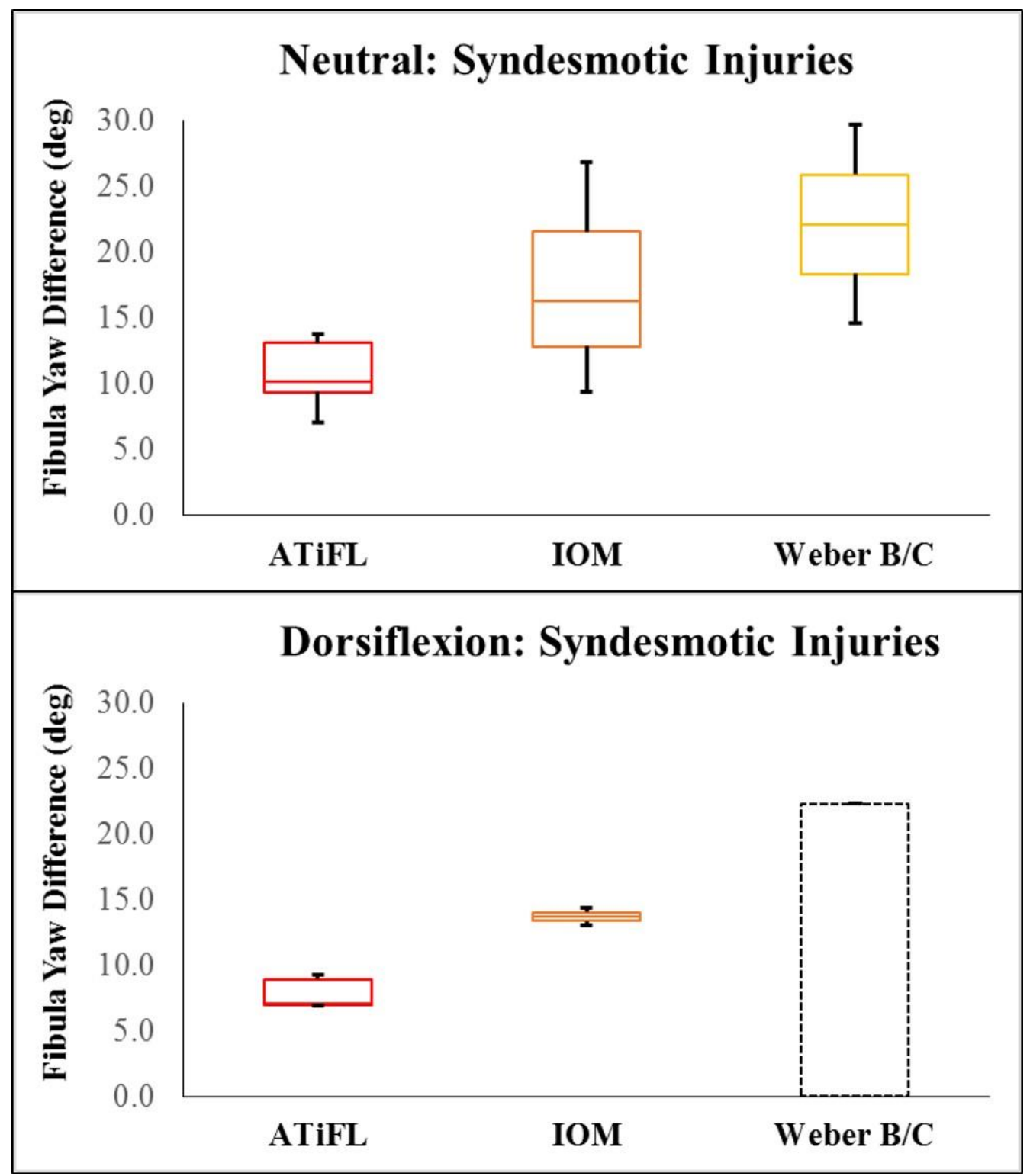

Figure 34: Injury intervals, relative to tibia and fibula yaw difference, for injuries to the syndesmosis (i.e., ATiFL, IOM, and Weber B/C fibula fractures. Sensitivity to initial ankle flexion posture is indicated among initially neutral

(top) and dorsiflexed (bottom) legs. Syndesmotic injury did not occur in initially plantarflexed specimens.

\subsection{Discussion and Conclusions}

Employing the injury timing methods as detailed in Section 3.2.5, the work in this chapter aimed to determine a kinematics-based injury occurrence interval, relative to external foot rotation, in the medial, lateral, and syndesmotic regions of the ankle (Objectives 2 and 3). Quantification of the 
tibiofibular interactions, specifically tibiofibular diastasis, before, during, and after syndesmotic injury was also a goal of this chapter (Objectives 2 and 3). Considerations were made to investigate the sensitivity of applied external foot rotation and tibiofibular diastasis to initial ankle flexion posture (Objective 3). The results presented in this chapter should have implications on clinical efforts for diagnosing and treating syndesmotic ankle sprains as well as on countermeasure efforts to mitigate injury (Objectives 3 and 4).

To develop injury risk functions for syndesmotic injuries, the boundary conditions detailed in this thesis should be applied to a rotation loading protocol, similar to that used by Wei et al. (2012b, 2010), where increments of foot rotation is applied until a single injury (indicated by changes in mechanical response data such as moment or strain drop) occurs and the leg is then offloaded. This allows for independent injuries to be caused and documented relative to applied rotation such that confounding of other injuries would not affect other load paths within the ankle. Experiments in this thesis were not conducted in this manner since multiple injuries do happen to athletes on the playing field and the sequence of ankle injuries (e.g., high occurrence of deltoid injury found in conjunction with syndesmotic injury), reacting to applied excessive foot rotation, would be of more utility to those diagnosing, treating, and mitigating syndesmotic ankle sprains.

Instead of developing statistical models (i.e., injury risk functions) which predict injury based on a measurable parameter such as external foot rotation, injury intervals were developed for the syndesmotic, medial, and lateral ankle regions in multiple initial ankle postures (Figure 26). These intervals indicate that syndesmotic and deltoid injuries occur, in the neutral ankle flexion posture, between $34^{\circ}$ and $56^{\circ}$ and $35^{\circ}$ and $48^{\circ}$ (interquartile ranges), respectively, of applied external foot rotation, whereas lateral injuries occurred between $44^{\circ}$ and $60^{\circ}$. For the plantarflexed legs (Figure 26), the syndesmotic injury interval is undefined since no syndesmotic injury was diagnosed in these tests. However, relative to external foot rotation, deltoid (between $22^{\circ}$ and $34^{\circ}$ ) and lateral (between $22^{\circ}$ and $37^{\circ}$ ) injuries occurred earlier in the rotation cycle in plantarflexed legs compared to neutral. For the dorsiflexed legs, despite a consistent incidence in the sample compared to neutral (Table 14), syndesmotic injury occurred slightly later in the rotation cycle, between $46^{\circ}$ and $56^{\circ}$ of ER, than in neutral (Figure 26). Deltoid (between $43^{\circ}$ and $60^{\circ}$ ) and lateral (between 
$60^{\circ}$ and $66^{\circ}$ ) injury in the dorsiflexed legs also occurred later in the rotation cycle compared to neutral.

Frank tibiofibular diastasis, indicated by lateral fibula displacement, was examined previously on radiographs to determine the presence of syndesmotic injury (Dattani et al., 2008; Edwards and DeLee, 1984; Harper and Keller, 1989; Rammelt et al., 2008; Sclafani, 1985). However, syndesmotic injury can be present without frank diastasis (Edwards and DeLee, 1984; Nussbaum et al., 2001), such that tibiofibular clear space increases by as little as $2.3 \mathrm{~mm}$ after sectioning the ATiFL (Xenos et al., 1995). Clinical diagnosis techniques such as the squeeze, external rotation, and fibular anterior-posterior translation tests have been suggested to identify syndesmotic injury (Dattani et al., 2008). Yet, similar to radiographs (Hopkinson et al., 1990), the external rotation and squeeze tests are inconsistent for evaluating syndesmotic injury (Beumer et al., 2003, 2002). However, the fibular anterior-posterior translation test, introduced by Ogilvie-Harris and Reed (1994) in addition to anterior-posterior and lateral radiographs (Anderson et al., 2010; Xenos et al., 1995), may provide a solution to these diagnosis technique inconsistencies, thus leading to additional confirmations of syndesmotic ankle sprains. In Figure 27, Figure 28, and Figure 29, posterior fibula translation (-Dx) was consistently the largest component of the displacement vector between the tibia and fibula in neutral and dorsiflexed legs where syndesmotic injury occurred. The magnitude of the fibula displacement vector increased as the Dx component decreased (i.e., increasing posterior fibula translation). Medial-lateral and inferior-superior translation (Dy and Dz components of the fibula displacement vector) did contribute to the magnitude calculation, however the magnitude trace did not follow the Dy and Dz data traces as apparently as it did with the Dx trace (Figure 27, Figure 28, and Figure 29). Therefore, posterior fibula translation contributes most to fibular displacement relative to the tibia and should be investigated when diagnosing syndesmotic ankle sprains.

From Figure 27, Figure 28, and Figure 29, injury intervals for syndesmotic injuries only, relative to the magnitude of the fibular displacement vector, were generated in the neutral and dorsiflexed legs (Figure 33). These intervals indicate the fibula displacement caused within this particular sample of cadavers that induced syndesmotic injury (ATiFL and IOM ruptures and Weber B/C fibula fractures). These intervals show that fibula displacement is similar for legs initially in neutral 
and dorsiflexion at ATiFL and IOM injury. For the neutral posture, ATiFL injury occurred between 6.1 and $7.4 \mathrm{~mm}$ (interquartile range) and IOM injury occurred between 9.5 and $14.0 \mathrm{~mm}$; whereas, for the dorsiflexed posture, ATiFL injury occurred between 6.2 and $6.9 \mathrm{~mm}$ and IOM injury occurred between 10.4 and $19.2 \mathrm{~mm}$. The small interquartile range for ATiFL injury compared to IOM injury can possibly be attributed to the structure of each ligament. The ATiFL is a taut ligament spanning the surfaces of the lateral malleolus and the distal-lateral aspect of the tibia with limited range of allowable fibula displacement from the tibia, whereas the IOM is a long, less taut structure permitting more compliance between the tibia and fibula (Beumer et al., 2002; Golanó et al., 2010; Kaumeyer and Malone, 1980; Skraba and Greenwald, 1984).

From Figure 30, Figure 31, and Figure 32, injury intervals for syndesmotic injuries only, relative to the difference between tibia and fibula yaw, were generated in the neutral and dorsiflexed legs (Figure 34). These intervals indicate the tibiofibular yaw difference caused within this particular sample of cadavers that induced syndesmotic injury (ATiFL and IOM ruptures and Weber B/C fibula fractures). These intervals show that tibiofibular yaw difference slightly decreases for legs initially in dorsiflexion compared to those in neutral at ATiFL and IOM injury. For the neutral posture, ATiFL injury occurred between $9.4^{\circ}$ and $13.1^{\circ}$ (interquartile range) and IOM injury occurred between $12.9^{\circ}$ and $21.6^{\circ}$; whereas, for the dorsiflexed posture, ATiFL injury occurred between $7.0^{\circ}$ and $8.9^{\circ}$ and IOM injury occurred between $13.4^{\circ}$ and $14.1^{\circ}$.

Tibiofibular diastasis has been defined previously as lateral displacement of the fibula from the tibia (Dattani et al., 2008; Edwards and DeLee, 1984; Harper and Keller, 1989; Rammelt et al., 2008; Sclafani, 1985). However, this study found that posterior fibula translation from the tibia is the largest component of fibula displacement before, during, and after syndesmotic injury (Figure 27, Figure 28, and Figure 29). In addition to the posterior fibula translation, this study found that the difference between tibia and fibula yaw rotation also increases before, during, and after syndesmotic injury (Figure 30, Figure 31, and Figure 32). Therefore, tibiofibular diastasis should be thought of as a combination of both fibula linear (translation) and angular (rotation) displacement from the tibia. The posterior fibula translation and tibiofibular yaw rotation difference observed in the cadavers for this study induces ATiFL and IOM injuries, with propagation into fibula fracture, after excessive external foot rotation. Injuries to the posterior 
tibiofibular structures (PTiFL or posterior IOM) were not diagnosed in the cadavers tested in this study (Table 14). This indicates that the syndesmosis joint opens anteriorly, under the experimental boundary conditions used in this study, during external foot rotation to induce tibiofibular diastasis (a combination of posterior fibula translation and tibiofibular yaw difference) and injures the anterior tibiofibular structures (ATiFL and anterior IOM). At ATiFL and IOM injury, fibula displacement magnitude was similar for both neutral and dorsiflexed legs (Figure 33); however, tibiofibular yaw difference (Figure 34) was less for dorsiflexed legs than for neutral. This could indicate that tibiofibular diastasis is influenced by the initial position and geometry of the ankle bones in dorsiflexion compared to neutral; such that, the syndesmotic ligaments can withstand similar linear elongations but less elongation resulting from fibula rotation in dorsiflexion than in neutral during similar applied external foot rotation.

As expected, tibiofibular diastasis (fibula displacement magnitude and tibia and fibula yaw difference) increased in both the neutral and dorsiflexed legs from ATiFL to IOM injury (Figure 33 and Figure 34). As the talus wedges further into the ankle mortise with increasingly applied external foot rotation, thus continually displacing the fibula from the tibia, the ATiFL is sacrificed first and then the IOM is sacrificed (Table 9, Table 10, and Table 12). Disrupting these ligamentous structures sequentially increases the displacement of the fibula relative to the tibia and rotates the fibula away from the tibia, thus opening the syndesmosis joint. Therefore, diastasis should increase as injuries propagate through the syndesmosis joint and then between the shafts of the tibia and fibula. However, with no occurrence of syndesmotic injury in plantarflexed legs (Table 11), despite being subject to the same preload and applied rotation, this suggests that the talus does not sufficiently wedge into the ankle mortise in plantarflexion as in neutral or dorsiflexion during applied foot rotation. Therefore, plantarflexion spares the syndesmotic ligaments as sufficient fibula displacement is not induced by the externally applied injury mechanism (external foot rotation) to initiate the internal injury mechanism (tibiofibular diastasis) and rupture the syndesmotic ligaments.

Anatomically, the talocrural joint acts as modified-hinge joint as the talar dome fits between the medial and lateral malleoli, which define the ankle mortise, and permits rotation of the foot to and from the leg in the sagittal plane (Norkus and Floyd, 2001). In neutral ankles, the talar dome is 
positioned coincident with the center of the tibial plafond and centered within the malleoli. As external foot rotation is applied, the calcaneus and talus rotate away from the medial malleolus, which puts the medial ankle ligaments (superficial and deep deltoid) in tension. If the medial ligaments are sacrificed, compliance of the talus within the ankle mortise increases and then forces the supero-lateral eminence of the talar dome to engage with the center of the lateral malleolus, which then displaces the fibula away from the tibia. As the fibula displaces from the tibia, the lateral ankle ligaments (ATaFL, CFL, and PTaFL) and syndesmotic ligaments (ATiFL and IOM) are placed into tension. From Table 9 and Table 10, at least one lateral injury was induced in 6 of 9 specimens and at least one syndesmotic injury was induced in 5 of 9 specimens. This suggests that the talar dome wedged into the ankle mortise sufficiently, during continually applied external foot rotation, in just over half of the specimens in neutral to induce enough tibiofibular diastasis which disrupted the syndesmotic ligaments. However, in the specimens where syndesmotic injury did not occur, deltoid injuries presumably offloaded the syndesmosis enough to not disrupt the syndesmotic ligaments or concomitant lateral injuries, occurring after the deltoid injuries, shared the talar dome loading on the fibula enough to protect the syndesmosis. Conversely, in the initially plantarflexed legs $\left(30^{\circ}\right)$, the supero-lateral eminence of the talar dome was initially displaced out of the ankle mortise, such that it did not engage the center of the lateral malleolus and only engaged the inferior tip. Even with the applied external foot rotation, the talus did not sufficiently wedge between the malleoli to displace the fibula and cause syndesmotic injury. Instead, the fibula displacements in the plantarflexed legs induced lateral ligament injury concomitant to deltoid injuries which presumably offloaded the syndesmosis. In the initially dorsiflexed legs $\left(15^{\circ}\right)$, the entire lateral talar dome was initially congruent with the center of the lateral malleolus. Therefore, as external foot rotation is applied, the deltoid ligaments are again sacrificed and the fibula is forced to displace from the tibia. With the entire lateral portion of the talar dome coincident within the ankle mortise, syndesmotic ligaments are forced into tension, as the fibula displaces from the tibia, and are eventually, consistently sacrificed. With excessive external foot rotation applied (internal tibia rotation up to $90^{\circ}$ ) in this thesis's experiments, lateral ligament injuries occurred after syndesmotic ligament injuries in the rotation cycle since the lateral structures were the only ligaments left to resist fibular displacements and rotations away from the tibia (diastasis) after sacrificing the syndesmotic ligaments. 
Though this is the first study to determine a kinematics-based injury interval for injuries in multiple ankle regions and among multiple initial ankle flexion postures, some limitations should be noted. Muscle forces were not considered in the experimental work in this thesis, though these forces could be a factor for syndesmotic injury re-creation. Sample size was limited ( $\mathrm{n}=9$ for neutral, $\mathrm{n}$ $=4$ for plantarflexion, and $\mathrm{n}=4$ for dorsiflexion) and anthropometry of the sample is not applicable to the greater human population. Cadaveric specimens were acquired to match average NFL player size and age as closely as possible (Manfred, 2014). Therefore, the injury intervals should not be considered comprehensive to the greater human population and are applicable to large males. With the limited sample size, the injury intervals indicated in this thesis lack statistical power and significance. Further tests could be performed, under the same external boundary conditions detailed in this study where the external injury mechanism (external foot rotation) induces the ankle internal injury mechanism (tibiofibular diastasis), to determine a statistically significant syndesmotic injury tolerance relative to external foot rotation or tibiofibular diastasis in the form of injury risk functions. The definition of tibiofibular diastasis (combination of fibula displacement magnitude and tibiofibular yaw difference) is limited in that the two measures are not combined into one cohesive measure to quantify tibiofibular diastasis on the whole. However, this is the first study, to the author's knowledge, to attempt to quantify tibiofibular diastasis with translational and rotational tibiofibular interactions during applied external foot rotation cadaveric experiments.

Despite the limitations of the injury intervals, the intervals do indicate that syndesmotic injury occurrence changes based on initial ankle flexion posture. Syndesmotic injury did not occur in initially plantarflexed specimens and, compared to the neutral, syndesmotic injury occurred later in the rotation cycle for initially dorsiflexed specimens. However, similar fibula displacement magnitude induced ATiFL and IOM injury among the neutral and dorsiflexed specimens (Figure 33), yet tibiofibular yaw difference was less for dorsiflexed specimens than neutral at ATiFL and IOM injury (Figure 34). Recall, tibiofibular diastasis was defined, in this thesis, as a combination of the fibula displacement magnitude and tibiofibular yaw difference. This is an interesting finding such that, though the dorsiflexed limbs withstood greater applied ER before syndesmotic injury occurred, the fibula translational displacement was similar at the occurrence of syndesmotic injury among neutral and dorsiflexed legs, but rotational displacement was less for dorsiflexed legs. This suggests linear tibiofibular compliance is similar among ankle postures before syndesmotic injury 
regardless of the amount of external foot rotation applied, with rotational compliance decreasing for dorsiflexed legs. Conversely, sparing the syndesmotic ligaments in plantarflexed legs comes at a cost. Deltoid and lateral ligaments are sacrificed earlier relative to applied external foot rotation in the plantarflexed legs (Figure 26), and the ATiFL is consistently sacrificed when excessive external foot rotation is combined with dorsiflexion (Table 14).

This is the first study for syndesmotic injury, to the author's knowledge, to quantify fibular motions in all anatomical directions and to determine injury intervals for injuries to multiple ankle regions during excessive external foot rotation experiments in cadavers. Intervals for syndesmotic, deltoid, and lateral ankle injuries are given for multiple ankle flexion postures relative to external foot rotation, which could be used in future countermeasure (e.g., footwear uppers and ankle braces) designs aimed at mitigating syndesmotic ankle sprains. This chapter details methods for quantifying fibular motions before, during, and after syndesmotic injury. For clinicians, this repository of tibiofibular interaction data should inform future diagnoses and interventions regarding syndesmotic ankle sprains (i.e., implementing lateral radiographs during diagnosis and investigating posterior fibula motion in the injured leg to compare to the uninjured leg). This tibiofibular kinematic interaction repository also elucidates the connection between the externally applied (external foot rotation) and internal (tibiofibular diastasis), within the ankle joint, injury mechanisms causing syndesmotic injury. In neutral and dorsiflexed legs, external foot rotation forces the supero-lateral eminence of the talar dome to displace the fibula from the tibia both in translation and rotation. This combination of posterior fibula translation and fibula yaw rotation, relative to the tibia, opens the syndesmosis joint anteriorly to induce injury in the ATiFL and IOM, with further propagation to fibula fracture, yet spares the posterior tibiofibular ligament (PTiFL). This finding is possible with the external boundary conditions implemented in this thesis on the cadaveric legs such that the fibula is unconstrained and foot compliance is allowed throughout the rotation cycle. Permitting fibula and foot compliance allows for natural ligamentous recruitment between the foot and ankle bones, thus inducing clinically significant syndesmotic injury under the externally applied (external foot rotation) and subsequent internal (tibiofibular diastasis) injury mechanisms within the ankle joint. 


\section{Chapter 5: Conclusions and Future Work}

\subsection{Implications for Syndesmotic Ankle Sprains}

The purpose of this thesis was to determine the injury pattern and sequence of ankle ligaments during applied excessive external foot rotation in varying ankle flexion postures: neutral, dorsiflexion, and plantarflexion. To accomplish this overall objective, this thesis first rigorously defined a coordinate system on individual foot and ankle bones so that relative six degree-offreedom bony motion could be measured throughout applied rotation (Objective 1). From the relative bony motion, ligament elongation within the ankle joint could be inferred, from which an injury event sequence among the medial, lateral, and syndesmotic ankle ligaments, induced by the imposed external foot rotation, was determined. Furthermore, the coordinate system definition detailed in this thesis allows for ankle motions to be related to clinical terms which can improve future understanding of ankle mechanical responses between those who test ankle mechanics and those who diagnose and treat ankle injuries (Objective 3).

With the geometrically defined coordinate system, a kinematic-based injury occurrence interval for ankle injuries was developed across the neutral, dorsiflexed, and plantarflexed postures (Objectives 2 and 3), in a matched-pair, contralateral leg study design. These injury intervals were developed for the proposed externally applied injury mechanism, external foot rotation, often attributed to syndesmotic ankle sprains. Furthermore, the external mechanism was related to the internal, within the ankle joint, injury mechanism of tibiofibular diastasis. A displacement vector was defined between the tibia and fibula and quantified, throughout the rotation cycle, to determine the magnitude of the linear (translational) displacement between the tibia and fibula before, during, and after syndesmotic injury. Contrary to some clinical studies, the posterior fibula displacement component was found to be the largest for both initially neutral and dorsiflexed specimens, when syndesmotic injury (ATiFL rupture) was induced. Tibiofibular rotation differences (yaw difference between tibia and fibula) were also quantified, throughout the rotation cycle, to determine the angular (rotational) displacement between the tibia and fibula before, during, and after syndesmotic injury. The internal, within the ankle joint, injury mechanism of tibiofibular diastasis is a combination of these linear and angular fibula displacements from the tibia. At ATiFL 
and IOM injury, the linear displacements (Figure 33) were similar for the neutral and dorsiflexed specimens, however the angular displacements (Figure 34) were less for dorsiflexed specimens than neutral. Therefore, the linear component of tibiofibular diastasis is potentially less sensitive to ankle posture than the angular component. The initial position of the talus in dorsiflexed legs, with the talar dome initially congruent with the proximal third of the lateral malleolus, may influence this sensitivity such that, at lower tibiofibular rotational differences syndesmotic injury is induced. However, the linear elongation needed to stretch the ATiFL and IOM to failure is similar for both neutral and dorsiflexed legs. Nevertheless, tibiofibular diastasis, induced by the external load of external foot rotation, should be considered as the main injury mechanism, with both linear and angular components, for syndesmotic ankle sprains by those who diagnose and treat syndesmotic injury (Objective 3).

Even with the geometrically defined coordinate system and six degree-of-freedom measurement of bony kinematics, these kinematics cannot be considered reliable if the external boundaries imposed on the leg are not functionally relevant nor a realistic reconstruction of the boundaries imposed on athletes on the playing field. Considerable experimental efforts were conducted in both non-injurious and injurious testing environments to determine a final set of experimental boundary conditions that reasonably balanced control and realistic joint mechanics. This evolution of experimental boundary conditions (Table 8) was designed to address deficiencies in the literature (Table 1) where fibula and foot compliance were not permitted during testing. Allowable compliance of the fibula and foot are important to induce proper syndesmotic injury re-creation. By imposing realistic joint mechanics, natural joint congruency is imposed and the axis of rotation within the leg is permitted to adjust during applied foot rotation; these are important for prompting natural ligament recruitment within the ankle reacting to the applied foot rotation. From this natural re-creation of osteoligamentous syndesmotic injury (i.e., both ligament and bone failures) a sequence of injury within the ankle in varying initial ankle postures was determined (Objectives 2 and 3). Injuries began in the medial ligaments, and then propagated into either the syndesmotic or lateral ligaments, depending on ankle flexion posture. For the neutral flexion posture, 5 of 9 specimens had propagation of medial injury into the syndesmosis, whereas further medial or concomitant lateral injuries were caused in the other neutral specimens, thus offloading the syndesmosis. However, for initially dorsiflexed specimens, injuries started in either the medial or 
syndesmosis regions of the ankle with further propagation into the syndesmosis (e.g., IOM rupture or fibula fracture) later in the rotation cycle. For initially plantarflexed specimens, injuries started in either the medial or lateral regions of the ankle with further propagation into those regions, thus offloading the syndesmosis. These injury sequences should be informative for clinicians diagnosing and treating syndesmotic ankle sprains, especially the connection between deltoid and syndesmotic injury occurrences, such that deltoid injury should always be investigated if a syndesmotic ankle sprain is diagnosed pre-operatively (Objective 3).

Ultimately, the results in this thesis suggest syndesmotic ankle sprain occurrence is sensitive to initial ankle posture. Plantarflexion of the ankle, combined with external foot rotation, protected the syndesmotic ligaments. Syndesmotic injury occurred in specimens initially oriented in neutral or dorsiflexion, with a $56 \%$ occurrence in neutral specimens and a $100 \%$ occurrence in dorsiflexed specimens. This indicates that the talar dome does not sufficiently wedge into the intra-malleolar ankle mortise during external foot rotation when in plantarflexion as it does in neutral or dorsiflexion to induce fibular linear and rotational displacement from the tibia and, in turn, syndesmotic injury. However, protecting the syndesmosis comes at a cost, since the deltoid and lateral ankle ligaments were sacrificed at lower angles of external foot rotation when combined with plantarflexion.

The results detailed in this thesis were attained using the most realistic and compliant loading scheme on the leg among all studies in the literature, to the author's knowledge. A link between the externally applied injury mechanism (external foot rotation) and the internal, within the ankle joint, injury mechanism (tibiofibular diastasis) was determined with the methods detailed in this thesis. As external foot rotation is applied, the talus engages with the lateral malleolus to displace the fibula from the tibia linearly and angularly, which combine to induce tibiofibular diastasis in the syndesmosis joint. This diastasis opens the syndesmosis joint anteriorly and induces ATiFL and anterior IOM injury with possible propagation to fibula fracture. This link would not have been possible without implementing the external boundaries surrounding the leg detailed in this thesis. These external boundaries left the fibula unconstrained and permitted natural foot compliance (e.g., calcaneus free to invert and evert), which is the first study to do so to the author's knowledge during excessive external foot rotation experiments on cadavers. These boundaries 
should be adapted for any future experiments aiming to re-create syndesmotic ligament injury such that the internal injury mechanism, tibiofibular diastasis, can be realistically re-created within the ankle during application of the external injury mechanism, external foot rotation. These experimental methods will be beneficial for future studies aiming to quantify syndesmotic ankle sprain tolerances to either the external or internal injury mechanism.

\subsection{Key Assumptions and Limitations}

A kinematic predictor of injury was assumed for the research detailed in this thesis and muscle forces (e.g., Achilles tendon load on the calcaneus) were not considered. However, these muscle loads, especially the Achilles tendon load, could affect syndesmotic injury occurrence and causation and the sensitivity of injury occurrence to these loads should be investigated in the future. Significant experimental effort was made to induce proper bone motion and subsequent ligament recruitment, since syndesmotic ankle sprains are induced by a subtle kinematic phenomenon (tibiofibular diastasis) where the talus wedges into the intra-malleolar ankle mortise. However, a compressive preload was placed on each leg to simulate a dynamic, weight-bearing scenario similar to those experienced by athletes on-field (Wei et al., 2012a, 2010). With no muscle forces, a kinetic predictor of injury is unreliable. To limit inertial effects, legs were rotated at a rate lower than those experienced by athletes on-field, and a sensitivity analysis to the rate of rotation was not performed. These assumptions should be taken into account when considering the data included in this thesis in the future.

Cadaveric specimens, especially those of a large size and young age at death, are scarce commodities. Therefore, the minimum possible sample of specimens was tested to complete the objectives of this thesis. This sample of specimens was pragmatically chosen by the experimenters to utilize specimens that were available, yet still matched anthropometric specifications (Manfred, 2014), so that an efficient experimental study was conducted and deliverables were met. However, this limitation is somewhat mitigated by the use of matched-pair, contralateral legs such that more direct correlations of injury incidence sensitivity to initial ankle flexion posture can be ascertained. The applicability of this study should be taken into consideration where injury intervals and diastasis quantifications presented were generated from a sample of cadavers intentionally aimed 
at matching the average NFL player (Manfred, 2014), and therefore should not be considered to be comprehensive for the human population.

Experimental methods in this thesis detail several iterations of external boundaries applied to the leg to accurately and realistically re-create syndesmotic ankle sprains in cadavers. These iterations were completed to find a balance between functionally relevant and mechanically tractable boundaries applied to the leg. This confounding of boundary conditions should be considered when interpreting the kinematic and injury data results presented in this thesis. However, the changes in boundary conditions were important to inform any future experiments aiming to re-create ligamentous syndesmotic ankle sprains and determine ankle tolerances to measurable stimuli. $\mathrm{BC}: \mathrm{C}, \mathrm{BC}: \mathrm{D}$, and $\mathrm{BC}: \mathrm{E}$ (Table 8) represent, in multiple ankle flexion postures, the closest representation of boundary conditions, compared to previous studies, imposed on athlete's during play. These boundary conditions are functionally relevant and mechanically tractable, such that resulting bone kinematics can be measured during ligamentous syndesmotic injury re-creation. As an attempt to quantify tibiofibular diastasis with both translational and rotational tibiofibular interactions, the definition of tibiofibular diastasis (combination of fibula linear displacement magnitude and tibiofibular yaw difference) detailed in this thesis is limited in that rotational and translational displacements are not combined into one cohesive measure for tibiofibular diastasis. Instead, the linear and angular fibula displacements from the tibia act as complimentary measures (Figure 27, Figure 28, Figure 29, Figure 30, Figure 31, and Figure 32) to quantify the overall diastasis between the tibia and fibula before, during, and after syndesmotic injury for the sample of cadavers tested. Nevertheless, this is the first study, to the author's knowledge, to attempt to quantify tibiofibular diastasis in such a way during applied external foot rotation cadaveric experiments. Further studies should investigate the viability of this tibiofibular diastasis definition.

Despite the assumptions and limitations, the results of this thesis represent a significant contribution to improved understanding of ankle mechanics during syndesmotic injury for sizable, non-senescent males. Intervals of injuries, relative to external foot rotation, for syndesmotic, deltoid, and lateral ankle injuries, induced during testing, are given for potential use in the future as design limits for countermeasures to mitigate syndesmotic ankle sprains. Important tibiofibular kinematic interactions before, during, and after syndesmotic injury were also quantified in this 
thesis which will be of future use for clinicians when diagnosing and treating syndesmotic ankle sprains. Intervals of injury occurrence relative to these tibiofibular kinematic interactions (i.e., tibiofibular diastasis) were also given in this thesis. These intervals (relative to external foot rotation, fibula displacement, and tibiofibular rotation differences) present a direct link between the injury mechanisms of syndesmotic ankle sprains, both externally applied (external foot rotation) and the resultant internal load applied to the ankle syndesmosis joint (tibiofibular diastasis) Ultimately, the kinematic-based injury occurrence intervals, in conjunction with the injury sequence data, will be implemented to improve injury-predictive capabilities of a finite element computational model (Mane, 2016; Mane et al., 2015; Nie et al., 2017b, 2017a, 2016b, 2016a), which should be used as a design tool for any future countermeasure or injury mitigation technique.

\subsection{Future Work}

\subsubsection{Experimental and Computational Improvements}

Future cadaveric experimental work could investigate the effects of eversion and inversion on the occurrence of syndesmotic injury, with the evolution of boundary conditions and injury event sequence determination developed in this thesis taken into account, since eversion has been cited as a contributor to syndesmotic injury (Funk, 2011; Lin et al., 2006; Wei et al., 2012b, 2010; Williams et al., 2007). Also, future cadaveric studies could be used to determine syndesmotic injury tolerances, in the form of injury risk functions, for all initial ankle postures for not only kinematic-based predictors of injury such as external foot rotation or tibiofibular diastasis but also kinetic-based predictors such as resultant torque in the leg or applied muscle forces. Combining all these initial ankle postures would complete a full design space for potential tolerance limits regarding syndesmotic ankle sprains. However, predictive statistical models are difficult to define for confounding ligamentous injuries. This should be taken into account when performing future cadaveric experiments, such that the loading protocol and external boundaries implemented in this thesis are adapted to incremental rotational loadings (Wei et al., 2012b, 2010) to induce independent, yet realistic syndesmotic injury events. 
In parallel to the experimental efforts detailed in this thesis, computational modeling improvements were also completed (Mane, 2016; Mane et al., 2015; Nie et al., 2017b, 2017a, 2016b, 2016a) to study syndesmotic ankle sprain mechanics. The experimental kinematic and injury data detailed in this thesis was used to validate this finite element model, with the goal of creating a biofidelic model for predicting syndesmotic ankle sprains (Figure 35). To fully validate this computational foot and ankle surrogate, the injury intervals relative to external foot rotation and tibiofibular diastasis and the full injury sequences (i.e., which ligament fails first), detailed in this thesis, should be implemented (Objective 4). Future cadaveric experiments intending to develop injury risk functions for injuries to particular ankle ligament regions could also be implemented into the computational model to create a completely biofidelic model for predicting syndesmotic ankle sprains. Instead of performing laborious cadaveric experiments, the finite element model could be used to investigate all possible effects of initial ankle posture, boundary conditions, and applied muscle forces on syndesmotic ankle sprain mechanics.

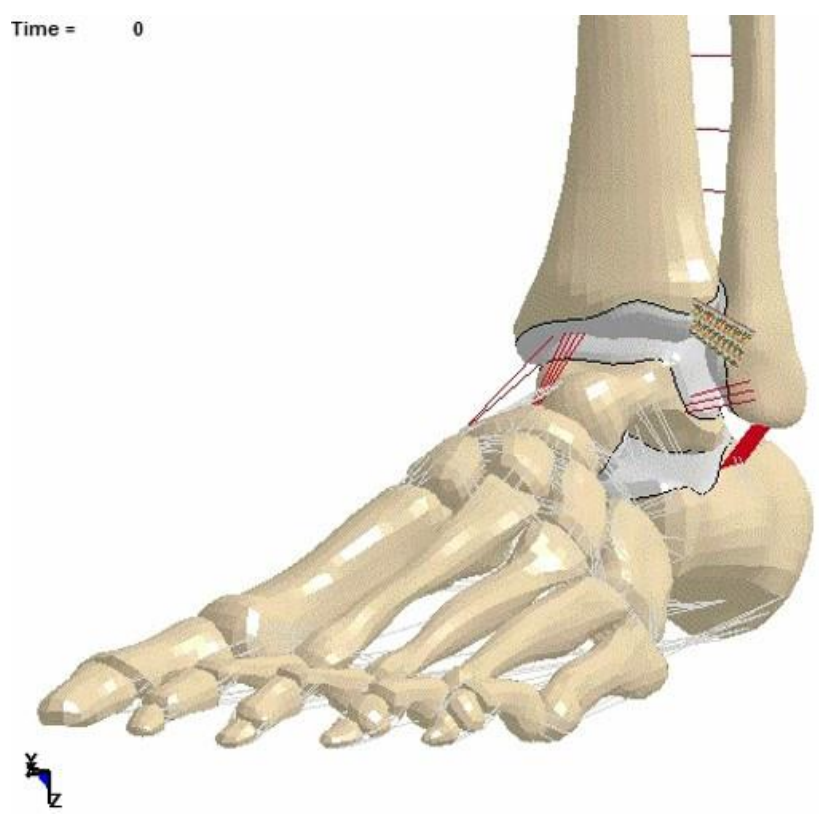

Figure 35: Computational finite element model ankle surrogate which should be used to test the viability of future countermeasure designs aimed at mitigating syndesmotic ankle sprains. 


\subsubsection{Countermeasure Design}

For future syndesmotic ankle sprain countermeasure or injury mitigation designs (Objective 4), dorsiflexion should be avoided since syndesmotic injury occurred in all dorsiflexed specimens. In all initially dorsiflexed and some neutral legs, the talus sufficiently wedged into the ankle mortise causing tibiofibular diastasis which induced syndesmotic injury. However, this was not the case for initially plantarflexed legs such that the talus does not wedge into the ankle mortise the same way as in neutral or dorsiflexed legs even under the same imposed preload and external foot rotation. In the future, the research in this thesis suggests that future countermeasure designs (e.g., ankle braces or footwear uppers) should aim to induce ankle plantarflexion after a certain amount of excessive external foot rotation (before $35^{\circ}$; Figure 26). If the foot and ankle can be forced into plantarflexion before reaching this external foot rotation angle, on-field syndesmotic injury could be mitigated. Loads within the syndesmosis can be shared across the ankle brace or footwear upper, thus decreasing the amount of load concentrated in the ATiFL or IOM. This result comes at a cost, however, since deltoid and lateral structures are sacrificed at lower angles of external foot rotation when combined with plantarflexion (Figure 26). Plantarflexed legs subject to external foot rotation could be susceptible to other lateral and medial ankle injuries such that an ankle dislocation occurs. These medial and lateral ankle injuries could be debilitating to athletes similar to that of syndesmotic injury and should be taken into account for future countermeasure design.

Not only is reducing external foot rotation important, but tibiofibular diastasis should also be reduced to prevent syndesmotic ankle sprains. As the internal (within ankle joint) injury mechanism, tibiofibular diastasis, in this thesis (magnitude of displacement vector between tibia and fibula origins and tibiofibular yaw differences), was found to increase before and during syndesmotic injury in both the neutral and dorsiflexed legs (Figure 33 and Figure 34). Future countermeasures should limit this fibular displacement, both linearly and rotationally, in conjunction with inducing ankle plantarflexion after excessive external foot rotation (externally applied injury mechanism), to reduce the occurrence of syndesmotic injury. These countermeasure designs should limit posterior fibula translation since it was found to be the largest component of the fibula displacement vector (Figure 27, Figure 28, and Figure 29) and limit fibula yaw (Figure 30, Figure 31, and Figure 32) such that the syndesmosis joint is not permitted to open anteriorly. 
Therefore, an ankle brace or footwear upper which is designed to squeeze the tibia and fibula together, intending to limit lateral fibula displacement from the tibia, will not be sufficient to reduce the occurrence of syndesmotic ankle sprains. Instead, countermeasures should limit posterior fibula translation and fibula rotations away from the tibia, yet still permit natural muscle and Achilles tendon activation, in order to best protect the ankle from syndesmotic injury.

A biofidelic finite element model (Figure 35), validated to the ankle bone kinematics and ligament failure propagations detailed in this thesis (Figure 26), can be implemented as an ankle surrogate in the future (Mane, 2016; Mane et al., 2015; Nie et al., 2017b, 2017a, 2016b, 2016a), thus reducing the need for cadaveric testing. This validated ankle surrogate has the capability to investigate the effects of taping/braces, parallel load paths, shoe plate compliance, and cleat-turf interactions on syndesmotic injury. This could include a 'sweet spot' of ankle plantarflexion (i.e., incremental degrees of ankle plantarflexion applied) during external foot rotation where torque within the leg is limited, yet syndesmotic injury is mitigated and medial and lateral ligament injury is reduced. These changes in boundary conditions surrounding the foot and ankle are ones that the specific athlete can control, therefore their effects can be investigated on an individual basis in the future. Understanding the effects of the boundary changes is a crucial step towards creating effective injury mitigation countermeasures, as evidenced by the evolution of boundary conditions used in the experimental work detailed in this thesis (Table 8). The validated finite element model can be utilized to implement any of these boundary conditions in real-time to test a new brace, cleat plate, or shoe upper technology. Ultimately, shoe and cleat designers as well as clinicians and athletic trainers can implement the finite element model to design new cleat plates, shoe uppers, ankle braces, or ankle taping techniques that will meet the ankle injury occurrence limits detailed in this thesis and prevent syndesmotic ankle sprains in the future. 


\section{References}

Allard, P., Cappozzo, A., Lundberg, A., Vaughan, C., 1997. Three-Dimensional Analysis of Human Locomotion. John Wiley \& Sons, Inc.

Anderson, R.B., Hunt, K.J., McCormick, J.J., 2010. Management of common sports-related injuries about the foot and ankle. J. Am. Acad. Orthop. Surg. 18, 546-556.

Barker, H.B., Beynnon, B.D., Renström, P. a. F.H., 1997. Ankle injury risk factors in sports. Sports Med. 23, 69-74.

Bartonicek, J., 2003. Anatomy of the tibiofibular syndesmosis and its clinical relevance. Surg. Radiol. Anat. 25, 379-386. doi:10.1007/s00276-003-0156-4

Bate, R.R., Mueller, D.D., White, J.E., 1971. Fundamentals of astrodynamics. Dover Publications, New York, NY.

Beumer, A., 2007. Chronic instability of the anterior syndesmosis of the ankle (Ph.D. Dissertation). Erasmus University Rotterdam, Rotterdam, The Netherlands.

Beumer, A., Swierstra, B.A., Mulder, P.G., 2002. Clinical diagnosis of syndesmotic ankle instability: evaluation of stress tests behind the curtains. Acta Orthop. Scand. 73, 667669.

Beumer, A., van Hemert, W.L.W., Swierstra, B.A., Jasper, L.E., Belkoff, S.M., 2003. A Biomechanical Evaluation of Clinical Stress Tests for Syndesmotic Ankle Instability. Foot Ankle Int. 24, 358-363. doi:10.1177/107110070302400410

Bloemers, F.W., Bakker, F.C., 2006. Acute Ankle Syndesmosis Injury In Athletes. Eur. J. Trauma 32, 350-356. doi:10.1007/s00068-006-1001-3

Bonnin, J., 1970. Injuries to the ankle. Hafner Publishing Company, Darien, Connecticut, USA.

Boytim, M.J., Fischer, D.A., Neumann, L., 1991. Syndesmotic ankle sprains. Am. J. Sports Med. 19, 294-298. doi:10.1177/036354659101900315

Camacho, D.L.A., Ledoux, W.R., Rohr, E.S., Sangeorzan, B.J., Ching, R.P., 2002. A threedimensional, anatomically detailed foot model: A foundation for a finite element simulation and means of quantifying foot-bone position. J. Rehabil. Res. Dev. Wash. 39, 401-10.

Campbell, K.J., Michalski, M.P., Wilson, K.J., Goldsmith, M.T., Wijdicks, C.A., LaPrade, R.F., Clanton, T.O., 2014. The Ligament Anatomy of the Deltoid Complex of the Ankle: A Qualitative and Quantitative Anatomical Study: J. Bone Jt. Surg.-Am. Vol. 96, e62-1-10. doi:10.2106/JBJS.M.00870

Cappozzo, A., Cappello, A., Croce, U.D., Pensalfini, F., 1997. Surface-marker cluster design criteria for 3-D bone movement reconstruction. IEEE Trans. Biomed. Eng. 44, 11651174. doi:10.1109/10.649988

Cappozzo, A., Della Croce, U., Leardini, A., Chiari, L., 2005. Human movement analysis using stereophotogrammetry: Part 1: theoretical background. Gait Posture 21, 186-196. doi:10.1016/j.gaitpost.2004.01.010

Cole, G.K., Nigg, B.M., Ronsky, J.L., Yeadon, M.R., 1993. Application of the Joint Coordinate System to Three-Dimensional Joint Attitude and Movement Representation: A Standardization Proposal. J. Biomech. Eng. 115, 344-349. doi:10.1115/1.2895496

Dattani, R., Patnaik, S., Kantak, A., Srikanth, B., Selvan, T.P., 2008. Injuries to the tibiofibular syndesmosis. Bone Jt. J. 90, 405-410. 
de Asla, R.J., Wan, L., Rubash, H.E., Li, G., 2006. Six DOF in vivo kinematics of the ankle joint complex: Application of a combined dual-orthogonal fluoroscopic and magnetic resonance imaging technique. J. Orthop. Res. 24, 1019-1027. doi:10.1002/jor.20142

Dias, L.S., Foerster, T., 1974. Traumatic Lesions of the Ankle Joint: The Supination-External Rotation Mechanism. Clin. Orthop. 100, 219-224.

Edwards, G.S., DeLee, J.C., 1984. Ankle diastasis without fracture. Foot Ankle 4, 305-312.

Eppinger, R., Sun, E., Bandak, F., Haffner, M., Khaewpong, N., Maltese, M., Kuppa, S., Nguyen, T., Takhounts, E., Tannous, R., others, 1999. Development of improved injury criteria for the assessment of advanced automotive restraint systems-II. Natl. Highw. Traffic Saf. Adm. 1-70.

Fioretti, S., Cappozzo, A., Lucchetti, L., 1997. Joint kinematics. Wiley \& Sons, Chichester, West Sussex, United Kingdom.

Fritschy, D., 1989. An unusual ankle injury in top skiers. Am. J. Sports Med. doi:10.1177/036354658901700223

Funk, J.R., 2011. Ankle injury mechanisms: Lessons learned from cadaveric studies. Clin. Anat. 24, 350-361. doi:10.1002/ca.21112

Gerber, J.P., Williams, G.N., Scoville, C.R., Arciero, R.A., Taylor, D.C., 1998. Persistent disability associated with ankle sprains: a prospective examination of an athletic population. Foot Ankle Int. 19, 653-660.

Golanó, P., Vega, J., de Leeuw, P.A.J., Malagelada, F., Manzanares, M.C., Götzens, V., van Dijk, C.N., 2010. Anatomy of the ankle ligaments: a pictorial essay. Knee Surg. Sports Traumatol. Arthrosc. 18, 557-569. doi:10.1007/s00167-010-1100-X

Gray, H., 1918. Anatomy of the Human Body, 20th ed. Lea \& Febiger, Philadelphia, PA, USA.

Green, C., Fitzpatrick, C., FitzPatrick, D., Stephens, M., Quinlan, W., Flavin, R., 2011. Definition of Coordinate System for Three-Dimensional Data Analysis in the Foot and Ankle. Foot Ankle Int. 32, 193-199. doi:10.3113/FAI.2011.0193

Guise, E.R., 1976. Rotational ligamentous injuries to the ankle in football. Am. J. Sports Med. 4, 1-6. doi:10.1177/036354657600400101

Harper, M.C., Keller, T.S., 1989. A Radiographic Evaluation of the Tibiofibular Syndesmosis. Foot Ankle 10, 156-160. doi:10.1177/107110078901000308

Hintermann, B., 1999. Biomechanics of the unstable ankle joint and clinical implications. Med. Sci. Sports Exerc. 31, S459-69. doi:10.1097/00005768-199907001-00007

Hopkinson, W.J., Pierre, P.S., Ryan, J.B., Wheeler, J.H., 1990. Syndesmosis sprains of the ankle. Foot Ankle 10, 325-330.

Houglum, P., Bertoti, D., 2012. Brunnstrom's Clinical Kinesiology, 6th ed. Philadelphia, PA, USA.

Hughes, J.L., Weber, H., Willenegger, H., Kuner, E.H., 1979. Evaluation of Ankle Fractures: Non-operative and Operative Treatment. Clin. Orthop. 138, 111-119.

Husfeldt, E., 1937. Significance of roentgenography of ankle joint in oblique projection in malleolar fractures. Hospitalstid 80, 788.

Jones, M.H., Amendola, A., 2007. Syndesmosis Sprains of the Ankle: A Systematic Review. Clin. Orthop. 455, 173-175. doi:10.1097/BLO.0b013e31802eb471

Kaumeyer, G., Malone, T., 1980. Ankle injuries: anatomical and biomechanical considerations necessary for the development of an injury prevention program. J. Orthop. Sports Phys. Ther. 1, 171-177. 
Kent, R.W., Funk, J.R., 2004. Data Censoring and Parametric Distribution Assignment in the Development of Injury Risk Functions from Biochemical Data. SAE Technical Paper.

Kent, R.W., Lievers, W.B., Riley, P.O., Frimenko, R.E., Crandall, J.R., 2014. Etiology and Biomechanics of Tarsometatarsal Injuries in Professional Football Players. Orthop. J. Sports Med. doi:10.1177/2325967114525347

Lauge-Hansen, N., 1950. FRACTURES OF THE ANKLE: II. Combined Experimental-Surgical and Experimental-Roentgenologic Investigations. Arch. Surg. 60, 957-985. doi:10.1001/archsurg.1950.01250010980011

Leardini, A., O'connor, J.J., Catani, F., Giannini, S., 2000. The role of the passive structures in the mobility and stability of the human ankle joint: a literature review. Foot Ankle Int. 21, 602-615.

Lessley, D., Shaw, G., Riley, P., Forman, J., Crandall, J., 2011. Assessment and validation of a methodology for measuring anatomical kinematics of restrained occupants during motor vehicle collisions. J Biosens Bioelectron S 1, 2.

Lin, C.-F., Gross, M.T., Weinhold, P., 2006. Ankle Syndesmosis Injuries: Anatomy, Biomechanics, Mechanism of Injury, and Clinical Guidelines for Diagnosis and Intervention. J. Orthop. Sports Phys. Ther. 36, 372-384. doi:10.2519/jospt.2006.2195

Lundberg, A., Svensson, O.K., 1993. The axes of rotation of the talocalcaneal and talonavicular joints. The Foot 3, 65-70. doi:10.1016/0958-2592(93)90064-A

Lundberg, A., Svensson, O.K., Bylund, C., Selvik, G., 1989. Kinematics of the ankle/foot complex - part 3: influence of leg rotation. Foot Ankle Int. 9, 304-309.

Mait, A., Donlon, J.P., Mane, A., Forman, J., Kent, R., 2015. Kinetics and Kinematics of the Ankle during Foot External Rotation, in: 39th Annual Meeting of the American Society of Biomechanics. Presented at the American Society of Biomechanics, Columbus, OH, USA.

Mait, A.R., Donlon, J.P., Nie, B., Forman, J.L., Anderson, R., Cooper, M.T., Kent, R.W., 2017a. Foot Flexion Alters Ankle Injury Patterns and Tolerance during Forced External Rotation, in: Proceedings of the Forty-Fourth International Workshop. Presented at the Injury Biomechanics Research, Washington, DC.

Mait, A.R., Mane, A., Forman, J.L., Donlon, J.P., Nie, B., Kent, R.W., 2017b. Transient and long-time kinetic responses of the cadaveric leg during internal and external foot rotation. J. Biomech. 53, 196-200. doi:10.1016/j.jbiomech.2017.01.006

Mane, A., 2016. Representing Sub-failure Quasi-static Ligament Mechanics and Bone Kinematics in a Human Ankle Finite Element Model (M.S. Thesis). University of Virginia, Charlottesville, VA, USA.

Mane, A., Nie, B., Panzer, M.B., Donlon, J.P., Mait, A., Kent, R., 2015. Human Ankle Ligament Toe Region Identification through Inverse Finite Element Approach, in: 13th International Symposium on Computer Methods in Biomechanics and Biomedical Engineering (CMBBE). Presented at the Computer Methods in Biomechanics and Biomedical Engineering Conference, Montreal, Canada.

Manfred, T., 2014. Here's The Ideal Body Type For Every Sport. Bus. Insid.

Markolf, K.L., Schmalzried, T.P., Ferkel, R.D., 1989. Torsional Strength of the Ankle In Vitro: The Supination-External-Rotation Injury. Clin. Orthop. 246, 266-272.

McCollum, G.A., van den Bekerom, M.P.J., Kerkhoffs, G.M.M.J., Calder, J.D.F., van Dijk, C.N., 2012. Syndesmosis and deltoid ligament injuries in the athlete. Knee Surg. Sports Traumatol. Arthrosc. 21, 1328-1337. doi:10.1007/s00167-012-2205-1 
Michelson, J., Solocoff, D., Waldman, B., Kendell, K., Ahn, U., 1997. Ankle fractures. The Lauge-Hansen classification revisited. Clin. Orthop. 198-205.

Moore, K., Dalley, A., Agur, A., 2010. Clinically oriented anatomy, 6th ed. Lippincott, Williams \& Wilkins, Philadelphia, PA, USA.

Nie, B., Forman, J.L., Mait, A.R., Donlon, J.P., Kent, R.W., 2017a. Foot Position Shifts Injury Initiation among Ankle Ligaments during External Rotation, in: 2017 IRCOBI Conference Proceedings. Presented at the International Research Council on Biomechanics of Injury, Antwerp, Belgium.

Nie, B., Panzer, M.B., Forman, J.L., Mane, A., Mait, A.R., Donlon, J.-P., Kent, R.W., 2016a. A Fiber-based Modelling Approach of Ankle Ligaments in situ, in: 2016 IRCOBI Conference Proceedings. Presented at the International Research Council on the Biomechanics of Injury, Malaga, Spain.

Nie, B., Panzer, M.B., Mane, A., Mait, A.R., Donlon, J.-P., Forman, J.L., Kent, R.W., 2017 b. Determination of the in situ mechanical behavior of ankle ligaments. J. Mech. Behav. Biomed. Mater. 65, 502-512.

Nie, B., Panzer, M.B., Mane, A., Mait, A.R., Donlon, J.-P., Forman, J.L., Kent, R.W., 2016b. A framework for parametric modeling of ankle ligaments to determine the in situ response under gross foot motion. Comput. Methods Biomech. Biomed. Engin. 19, 1254-1265. doi:10.1080/10255842.2015.1125474

Nigg, B.M., Skarvan, G., Frank, C.B., Yeadon, M.R., 1990. Elongation and Forces of Ankle Ligaments in a Physiological Range of Motion. Foot Ankle 11, 30-40. doi:10.1177/107110079001100107

Norkus, S.A., Floyd, R.T., 2001. The anatomy and mechanisms of syndesmotic ankle sprains. J. Athl. Train. 36, 68.

Nussbaum, E.D., Hosea, T.M., Sieler, S.D., Incremona, B.R., Kessler, D.E., 2001. Prospective evaluation of syndesmotic ankle sprains without diastasis. Am. J. Sports Med. 29, 31-35.

Ogilvie-Harris, D.J., Reed, S.C., 1994. Disruption of the ankle syndesmosis: Diagnosis and treatment by arthroscopic surgery. Arthrosc. J. Arthrosc. Relat. Surg. 10, 561-568. doi:10.1016/S0749-8063(05)80015-5

Ogilvie-Harris, D.J., Reed, S.C., Hedman, T.P., 1994. Disruption of the ankle syndesmosis: Biomechanical study of the ligamentous restraints. Arthrosc. J. Arthrosc. Relat. Surg. 10, 558-560. doi:10.1016/S0749-8063(05)80014-3

Paul, J., 1992. Terminology and units (Deliverable No. 4 No. CEC Programme AIM), Project A2002: CAMARC-II.

Peña, F.A., Coetzee, J.C., 2006. Ankle Syndesmosis Injuries. Foot Ankle Clin. 11, 35-50. doi:10.1016/j.fcl.2005.12.007

Rammelt, S., Zwipp, H., Grass, R., 2008. Injuries to the Distal Tibiofibular Syndesmosis: an Evidence-Based Approach to Acute and Chronic Lesions. Foot Ankle Clin. 13, 611-633. doi:10.1016/j.fcl.2008.08.001

Rastegar, J., Miller, N., Barmada, R., 1980. An apparatus for measuring the load-displacement and load-dependent kinematic characteristics of articulating joints-applications to the human ankle joint. J. Biomech. Eng. 102, 208-213.

Roaas, A., Andersson, G.B.J., 1982. Normal Range of Motion of the Hip, Knee and Ankle Joints in Male Subjects, 30-40 Years of Age. Acta Orthop. Scand. 53, 205-208. doi:10.3109/17453678208992202 
Roach, K.E., Wang, B., Kapron, A.L., Fiorentino, N.M., Saltzman, C.L., Foreman, K.B., Anderson, A.E., 2016. In Vivo Kinematics of the Tibiotalar and Subtalar Joints in Asymptomatic Subjects: A High-Speed Dual Fluoroscopy Study. J. Biomech. Eng. 138, 091006.

Rose, J.D., Flanigan, K.P., Mlodzienski, A., 2002. Tibiofibular diastasis without ankle fracture: a review and report of two cases. J. Foot Ankle Surg. 41, 44-51.

Rubin, A., Sallis, R., 1996. Evaluation and diagnosis of ankle injuries. Am. Fam. Physician 54, 1609-1618.

Sclafani, S.J., 1985. Ligamentous injury of the lower tibiofibular syndesmosis: radiographic evidence. Radiology 156, 21-27.

Shaw, G., Parent, D., Purtsezov, S., Lessley, D., Crandall, J., Kent, R., Guillemot, H., Ridella, S.A., Takhounts, E., Martin, P., 2009. Impact Response of Restrained PMHS in Frontal Sled Tests: Skeletal Deformation Patterns Under Seat Belt Loading. Stapp Car Crash J. $53,1-48$.

Shin, J., Yue, N., Untaroiu, C.D., 2012. A Finite Element Model of the Foot and Ankle for Automotive Impact Applications. Ann. Biomed. Eng. 40, 2519-2531. doi:10.1007/s10439-012-0607-3

Siegler, S., Chen, J., Schneck, C.D., 1988. The Three-Dimensional Kinematics and Flexibility Characteristics of the Human Ankle and Subtalar Joints-Part I: Kinematics. J. Biomech. Eng. 110, 364-373. doi:10.1115/1.3108455

Skraba, J.S., Greenwald, A.S., 1984. The Role of the Interosseous Membrane on Tibiofibular Weightbearing. Foot Ankle 4, 301-304. doi:10.1177/107110078400400605

Svensson, O.K., Lundberg, A., Walheirn, G., Selvik, G., 1989. In vivo fibular motions during various movements of the ankle. Clin. Biomech. 4, 155-160.

Taylor, D.C., Englehardt, D.L., Bassett, F.H., 1992. Syndesmosis sprains of the ankle: The influence of heterotropic ossification. Am. J. Sports Med. doi:10.1177/036354659202000209

Teramoto, A., Kura, H., Uchiyama, E., Suzuki, D., Yamashita, T., 2008. Three-Dimensional Analysis of Ankle Instability after Tibiofibular Syndesmosis Injuries: A Biomechanical Experimental Study. Am. J. Sports Med. 36, 348-352. doi:10.1177/0363546507308235

Villwock, M.R., Meyer, E.G., Powell, J.W., Haut, R.C., 2009. External Rotation Ankle Injuries: Investigating Ligamentous Rupture. Presented at the ASME 2009 Summer Bioengineering Conference, American Society of Mechanical Engineers, pp. 253-254. doi:10.1115/SBC2009-206210

Waterman, B.R., Belmont, P.J., Cameron, K.L., DeBerardino, T.M., Owens, B.D., 2010. Epidemiology of Ankle Sprain at the United States Military Academy. Am. J. Sports Med. doi:10.1177/0363546509350757

Wei, F., Hunley, S.C., Powell, J.W., Haut, R.C., 2011. Development and Validation of a Computational Model to Study the Effect of Foot Constraint on Ankle Injury due to External Rotation. Ann. Biomed. Eng. 39, 756-765. doi:10.1007/s10439-010-0234-9

Wei, F., Meyer, E.G., Braman, J.E., Powell, J.W., Haut, R.C., 2012a. Rotational stiffness of football shoes influences talus motion during external rotation of the foot. J. Biomech. Eng. 134, 041002.

Wei, F., Post, J.M., Braman, J.E., Meyer, E.G., Powell, J.W., Haut, R.C., 2012b. Eversion during external rotation of the human cadaver foot produces high ankle sprains. J. Orthop. Res. 30, 1423-1429. doi:10.1002/jor.22085 
Wei, F., Villwock, M.R., Meyer, E.G., Powell, J.W., Haut, R.C., 2010. A Biomechanical Investigation of Ankle Injury Under Excessive External Foot Rotation in the Human Cadaver. J. Biomech. Eng. 132, 091001. doi:10.1115/1.4002025

Williams, G.N., Jones, M.H., Amendola, A., 2007. Syndesmotic Ankle Sprains in Athletes. Am. J. Sports Med. 35, 1197-1207. doi:10.1177/0363546507302545

Wolfe, M.W., Uhl, T.L., Mattacola, C.G., McCluskey, L.C., 2001. Management of Ankle Sprains. Am. Fam. Physician 63, 93-104.

Wright, R.W., Barile, R.J., Surprenant, D.A., Matava, M.J., 2004. Ankle Syndesmosis Sprains in National Hockey League Players. Am. J. Sports Med. 32, 1-5. doi:10.1177/0363546504264581

Wu, G., Siegler, S., Allard, P., Kirtley, C., Leardini, A., Rosenbaum, D., Whittle, M., D D’Lima, D., Cristofolini, L., Witte, H., others, 2002. ISB recommendation on definitions of joint coordinate system of various joints for the reporting of human joint motion-part I: ankle, hip, and spine. J. Biomech. 35, 543-548.

Xenos, J.S., Hopkinson, W.J., Mulligan, M.E., Olson, E.J., Popovic, N.A., 1995. The tibiofibular syndesmosis. Evaluation of the ligamentous structures, methods of fixation, and radiographic assessment. J Bone Jt. Surg Am 77, 847-856.

Yamaguchi, S., Sasho, T., Kato, H., Kuroyanagi, Y., Banks, S.A., 2009. Ankle and Subtalar Kinematics during Dorsiflexion-Plantarflexion Activities. Foot Ankle Int.

doi:10.3113/FAI.2009.0361 